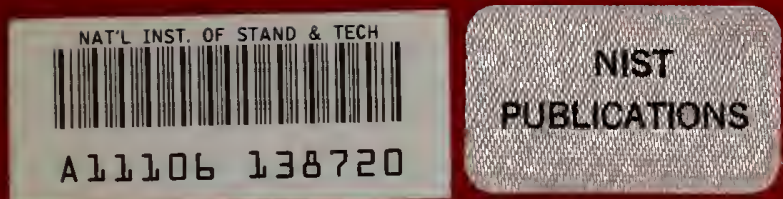

NBSIR 80-2018 (DOT)

A Third Appraisal of Methods for Estimating Self-Reaction Hazards

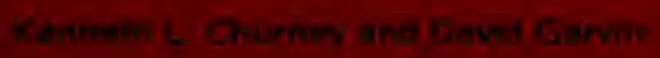
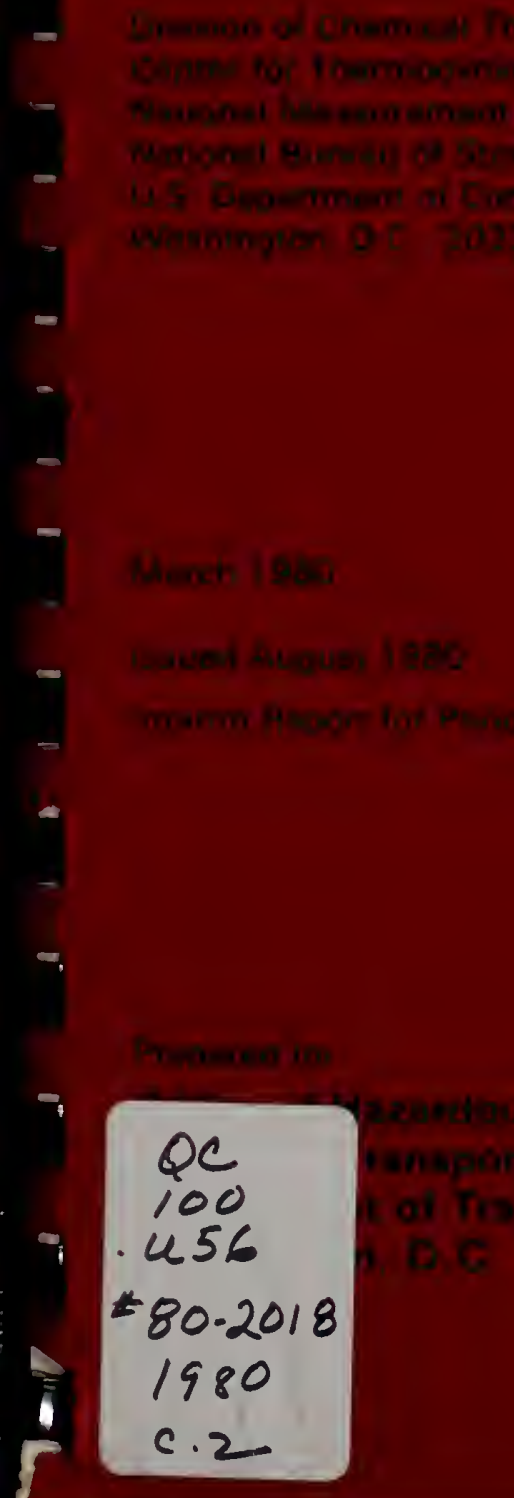

- 



\section{A THIRD APPRAISAL OF METHODS FOR ESTIMATING SELF-REACTION HAZARDS}

Kenneth L. Churney and David Garvin

Division of Chemical Thermodynamics

Center for Thermodynamics and Molecular Science

National Measurement Laboratory

National Bureau of Standards

U.S. Department of Commerce

Washington, D.C. 20234

March 1980

Issued August 1980

Interim Report for Period January 1976 - August 1978

Prepared for

Office of Hazardous Materials Operations

Materials Transportation Bureau

Department of Transportation

Washington, D.C. 20590

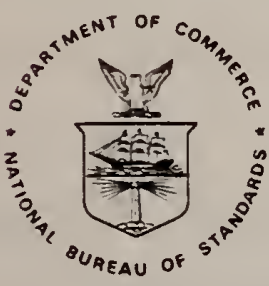

U.S. DEPARTMENT OF COMMERCE, Philip M. Klutznick, Secretary Luther H. Hodges, Jr., Deputy Secretary Jordan J. Baruch, Assistant Secretary for Productivity, Technology, and Innovation NATIONAL BUREAU OF STANDARDS, Ernest Ambler, Director 

Abstract

A study has been made of thermal explosion theory and thermal hazard tests. From this definitions have been developed, suitable for use in a transportation context, of thermally unstable materials-package configurations and thermal instability hazards. A thermally unstable materials package configuration is one:

(1) composed of substances that can decompose or react with the evolution of heat,

(2) for which a thermal mechanism of initiation of a temperature rise is present,

and (3) in which a resulting large increase in the temperature of the material can occur.

A thermal instability hazard exists if decomposition of the materialpackage configuration results in destruction of the package or neighboring objects or poses a safety threat. Typical destructions are explosion, rupture of package, and fire.

The properties of the material and package needed to predict the hazard are identified, and methods for their measurement, using heat conduction and adiabatic calorimetry, are analyzed. It is concluded that, for least ambiguous scale-up of results to bulk shipping conditions, measurements should be made on large samples of the material under conditions such that heat transfer within the sample is large in comparison to heat transfer between the sample and its environment. Tests, such as the explosion temperature test, should be carried out to check the scaled-up hazard predictions. 
An experimental study on three thermally unstable materials, nitrocellulose (I), m-azidobenzoic acid (II), and azidotriphenylmethane (III), was carried out using a differential scanning calorimeter (DSC). The procedure used is that of a proposed ASTM method of test for determining Arrhenius kinetic constants from the variation of the temperature of the maximum rate of heat evolution with scanning rate. Experimental results for activation energies, $\Delta E$, and prexponential factors, $A$; are: $\Delta E=$ (I) $166.9 \pm 6.1$, (II) $139.9 \pm 5.9$, and (III) $145.3 \pm 4.4 \mathrm{~kJ} \mathrm{~mol}^{-1}$; . $\log _{10}\left(\mathrm{~A}, \mathrm{~min}^{-1}\right)=$ (I) $18.0 \pm 0.7$, (II) $15.1 \pm 0.7$, and (III) $15.0 \pm 0.6$. A systematic error analysis clarifies and tends to support the test procedure. The main drawbacks to the test are its non-applicability to complex systems and the small temperature range of the data, which makes the extrapolation of results to lower temperatures uncertain.

\section{Key Words}

Adiabatic calorimetry, azidotriphenylmethane, decomposition reactions, differential scanning calorimetry, heat conduction calorimetry, kinetics of heat evolution, m-azidobenzoic acid, nitrocellulose, quantitative differential thermal analysis, thermal explosion theory, thermal hazards, thermal instability. 


\section{Foreword}

This report consists of two separate parts. Part I contains the conclusions and the main text, sections 1 to 5 . The main results of this study in context of previous work at NBS are given in sections 1 to 3 . A. nore complete and detailed summary of results is given in sections 4 and 5 .

Part II is the documentation on which. the conclusions are based, appendices A through C. Part II also contains a glossary of symbols, Appendix D, and the references, Appendix E. 
List of Tables... . . . . . . . . . . . . . v vii

List of Figures . . . . . . . . . . . . . . . . Ix

1. Introduction ................... 1

2. Summary of Previous Work and Comments . . . . . . . . 4

2.1. First Apparaisal of Methods for Estimating

Self-Reaction Hazards ............. 4

2.2. Second Appraisal of Methods . . . . . . . . . 5

2.3. Comments ................. . . 6

3. Summary of Present Work and Conclusions ......... . 8

4. Thermal Explosions . . . . . . . . . . . . 11

4.1. Introduction . . . . . . . . . . . . . 11

4.2. Models for Thermal Explosions ........... 13

4.2.1. Explosion Temperature Test. . . . . . . 14

4.2.2. Programmed Heating Test . . . . . . . 16

4.3. Thermal Explosion Theory . . . . . . . . . 17

4.3.1. The Isothermal (Uniform Temperature)

Sample .............. . . 17

4.3.2. Criteria for Explosion ........ 20

4.3.3. Extension to Non-Isothermal Samples and

Complex Kinetics . . . . . . . . 22

4.3.4. Programed Heating of Unstable

Materials ............. . 23

4.3.5. Conclusions .............. . . 24

4.3.6. Definition of Thermal Instability and a

Thermal Hazard Criterion. . . . . . . 28 
4.4. Thermal Explosion Experimentation and Self

Reaction Hazard Tests . . . . . . . . . . 31

4.4.1. Experiments on Explosions in Gases. . . . . 32

4.4.2. Experiments on Explosions in

Condensed Phases . . . . . . . . .

4.4.3. Conclusions Concerning Thermal Explosion

Experimentation ........... . 36

4.5. Hazard Tests . . . . . . . . . . . . 38

4.5.1. General Description of Field. . . . . . . 38

4.5.2. Thermal Sensitivity Tests......... 40

4.5.3. Adiabatic Calorimetry . . . . . . . . 41

4.5.4. Heat Conduction Calorimetry . . . . . 43

4.5.5. Conclusions Concerning Hazard Testing . . . 48

5. Experimental Work . . . . . . . . . . . . . 54

5.1. Introduction ... . . . . . . . . . 54

5.2. Summary and Assessment of Proposed ASTI

Test Method . . . . . . . . . . . . 56

5.3. Instrument Evaluation and Calibration ....... 61

5.4. Experimental Work. . . . . . . . . . . . 65

5.5. Discussion of Test Results. . . . . . . . . . 38

5.6. Conclusions Based on the Experiments and

Recommendations for Future Work . . . . . . . 102 Appendices and References-(Contents of Part II)

A. Remarks on Initial NBS Study . . . . . . . . . . . A.1-1

A.1. CHETAH Program . . . . . . . . . . . . . A.1-1

A.2. STULL-CRUISE Program and NFPA Hazard

Index . . . . . . . . . . . . . . . A. 2-1 
A.3. Summary of the Results of the Initial Study . . . A.3-1

A.4. Recent Developments ............. . A.4-1

B. Thermal Explosion Study Notes . . . . . . . . . . B.1-1

B.1. Description of the Thermal Explosion Process. . . B.1-1

B.2. Description of the Isothermal Mode1 ........ B.2-1

B.3. Steady State Analysis of the Isothermal Model . . B.3-1

B.4. Nonstationary Analysis of the Isothermal Model,

$f(n)$ Constant ................. B. . . . .

B.5. Nonstationary Analysis of the Isothermal Model,

$f(n)$ Variable . . . . . . . . . . . . B. $5-1$

B.6. Temperature Gradients in the Material, Various

Geometries ................ . B.6-1

B.7. Autocatalytic, Multiple, and Heterogeneous

Reactions ................ . B. $7-1$

B.8. Thermal Explosions in Linear Heating. . . . . . B.8-1

B.9. Experiments on Gaseous Reactions . . . . . . . B.9-1

B.10. Experimental Work on Explosives in the U.S.S.R.,

to 1970 . . . . . . . . . . . . . B.10-1

B.11. Hazard Evaluation Experimental Work : . . . . . B.11-1

C. Fundamental Paramter Tests ............. . C.1-1

c.1. Adiabatic Calorimetry .............. . C.1-1

C.2. Conduction Calorimetry Thermal Instability

Tests..................... C. $2-1$

C.3. Simplified Measurement Theory of Quantitative

Differential Analysis (QDTA) and Differential

Scanning Calorimetry (DSC) . . . . . . . . . C.3-1

C.3.1. Measurement Equations for QDTA ...... C.3-1 
C.3.2. QDTA and DSC Equations for

Kinetics ............. . C.3-7

C.3.3. Measurement Equations for DSC . . . . . C.3-14

C.3.4. Transfer Functions and Temperature

Calibration ............ C. . . . . 21

C.3.5. Determination of $\mathrm{K}$. . . . . . . . C.3-27

C.4. Instrument Calibration ........... C.4-1

C.4.1. Main Experimental Results ......... C.4-1

C.4.2. Analysis of Calibration Procedures. . . . C.4-12

C.5. Error Analysis for Four Assumptions . . . . . . C.5-1

C.5.1. The Value of $g\left(\eta_{d}\right)$ at Maximum $\mathrm{d} n / \mathrm{dt} . .$. C.5-1

C.5.2. Assumption of Constant Scanning Rate in

Evaluating $g\left(n_{\mathrm{d}}\right) \cdot . \cdot \cdot \cdot \cdot \cdot \cdot \cdot \cdot$.

C.5.3. Correction to Assumptions 1, 2 of

Table 11 . . . . . . . . . C.5-8

C.6. Closed Reusable Capsule ............ C.6-1

D. Table of Symbols . . . . . . . . . . . . . . D-1

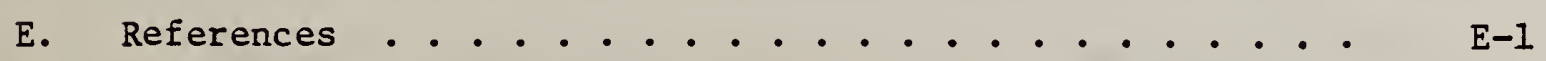


Table 1. Summary of High Pressure Cells for DSC Applications . . . . . . . . . . . . .

Table 2. m-Azidobenzoic Acid, Calculation of $\mathrm{T}_{\mathrm{d}} \cdot$. . .

Table 3. Azidotriphenylmethane, Calculation of $\mathrm{T}_{\mathrm{d}} \cdot$. .

Table 4. Nitrocellulose, Calculation of $\mathrm{T}_{\mathrm{d}}$. . . . . .

Table 5. Key to Tables 2, 3,4 ............ . 77, 73

Table 6. m-Azidobenzoic Acid, Kissinger Method. . . . . 85

Table 7. Azidotriphenylmethane, Kissinger Method. . . . . 86

Table 8. Nitrocellulose, Kissinger Method . . . . . . .

Table 9. Summary of Results on Kinetics of Heat

Evolution . . . . . . . . . . . . . .

Table 10. Comparison of Results . . . . . . . . . .

Table 11. List of Assumptions (Proposed ASTM Test

Method). . . . . . . . . . . . . .

Table 12. Effect of Error in Assumption 1 of Table 11 ..

Table 13. Effect of Error in Assumption 2 of Table

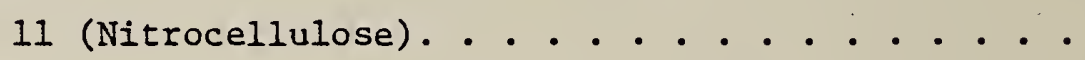

Table A-1 CHETAH Parameters and Classification of Compounds by Interpreters ............ A.4-4,5

Table $\mathrm{B}-1$ Induction Times and Reactant Consumption, Explosive States, $f(n)$ is Constant...... B. . . $4-4$

Table B-2 $(d \Theta / d t) \times\left[\tau_{\infty} / f\left(\eta_{m}\right)\right]$ versus $\theta$, Exponential Approximation ............. . . B. . . . . . Table B-3 Results of Tyler and Wesley [39] . . . . . . B.5-12 Table B-4 Mechanical Hazard Test Survey . . . . . . . B.11-5 
Table B-4a References, Comments, Table B-4. . . . . . . . B.11-6

Table B-5 Thermal Hazard Test Survey . . . . . . . . B.11-7,8

Table B-5a References and Comments for Table B-5. . . . . . B.11-9

Table C-1 Conduction Calorimetry Tests . . . . . . . . C.2-2

Table C-2 Details on Conduction Calorimetry Tests. . . . . C.2-3,4

Table C-3 Measurement Equations for QDTA .......... C.3-5

Table C-4 Baseline Treatment for Kinetics . . . . . . . . C.3-9

Table C-4a Definitions and Comments, Table C-4. . . . . . C.3-10

Table C-5 Equations for Simplified DSC Measurement

Analysis ................ . . . . 3-19

Table C-6 Temperature Calibration and Time Constant

Study ................. C.4-4,5

Table C-6a Key to Table C-6... . . . . . . . . . . . C.4-6,7

Table C-7 Energy Calibration .............. . C.4-11

Table C-8a Approximate Equations for DSC, Formulation of

Equations ............... . . C.4-15

Table C-8b Approximate Equations for DSC, Solution of

Entry 2, Table C-8a ............ C.4-16

Table C-9 Correction for Assumptions 1, 2 of Table 11. . . C.5-11 
Figure 1 Sketch of Cross Section of an Idealized Adiabatic Calorimeter . . . . . . . . . .

Figure 2 Sketch of Cross Section of an Idealized Heat Conduction Calorimeter . . . . . . . . . .

Figure 3 Sketch of Cross Section of an Idealized QDTA . . . . . . . . . . . . . . . . .

Figure $4 \quad$ Sketch of Cross Section of DSC Apparatus (Used in Experimental Work). . . . . . . . . . .

Figure 5 Sketch of Cross Section of DTA . . . . . . . .

Figure 6 Sketch of Exotherm of Polyacrylonitrile. . . . .

Figure 7 Sketch of Cross Section of Sample Containers . . .

Figure 8 Ordinate Signal versus Nominal Program

Temperature, m-Azidobenzoic Acid . . . . . . .

Figure 9 Ordinate Signal versus Nominal Program

Temperature, Azidotriphenylmethane . . . . . .

Figure 10 Ordinate Signal versus Nominal Program

Temperature, Nitrocellulose . . . . . . . .

Figure 11 Baseline Construction for Ordinate Signals . . .

Figure 12 Plot of $\log _{10} \mathrm{w}_{\mathrm{d}}$ result $1 / \mathrm{T}_{\mathrm{d}}$, m-Azidobenzoic

Acid . . . . . . . . . . . . . . .

Figure. 13 Plot of $\log _{10} w_{d}$ versus $1 / T_{d}$, Azidotriphenyl-. methane . . . . . . . . . . . . . .

Figure 14 Plot of $\log _{10} \mathrm{w}_{\mathrm{d}}$ versus $1 / \mathrm{T}_{\mathrm{d}}$, Nitrocellulose ...

Figure B-1 Semenov Diagram . . . . . . . . . . . . B.3-2

Figure B-2 Semenov Diagram; $f(n)=(1-n) . . . . . . . . \quad$ B. . . . 8 
Figure $B-3$ Sketch of $1 / \delta^{\prime}=$ a versus $\gamma=1 / B, n=1, \beta=0 . \quad B .5-14$

Figure $B-4$ sketch of $\theta_{\text {m }}\left(\theta\right.$ for $d \theta / d t=0$ ) versus $\delta^{\prime}$ (after

Merzhanov et al [42])........... . . . . . . . . .

Figure C-1 Sketch of Cross Section of QDTA Described by

Baxter [173] . . . . . . . . . . . C.3-2

Figure $\mathrm{C}-2$ Conductance Diagran for Figure $\mathrm{C}-1$. . . . . . C.3-4

Figure C-3 Simplified Sketch of DSC-2 Output Circuitry . . . C.3-17

Figure C-4 Sketch of Melting Endotherm . . . . . . . . . c.3-24

Figure C-5 Typical Plot of Onset-temperature of Fusion, $T_{\text {on }}(w)$,

versus Scanning Rate, w. ........... C.4-2

Figure C-6 Plot of Static Temperature Corrections, $\Delta \mathrm{T}_{\text {STAT }}$,

versus Program Temperature, $\mathrm{T}_{\mathrm{p}}$. . . . . . C.4-3

Figure C-7 Estimate of $\tau_{d}$ from Exotherm of Freezing of

Supercooled Indium ............. . C.4-9

Figure C-8 Heat Capacity of Aluminum Containers . . . . . C.4-13

Figure C-9 Sketch of Diametral Cross Section of Closed

Reusable Capsule .............. . C.5-11

Figure C-10 Sketch of Diametral Cross Section of Modified DSC

Holder Lids. . . . . . . . . . . . C.6-3 


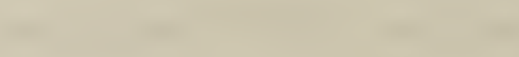




\section{Introduction}

The work reported here represents the third in a series of studies carried out at NBS between January 1974 and August 1978 to develop a clear definition of and quantitative test methods for thermal instability of chemicals under bulk shipping (and storage) conditions.

This third appraisal is (1) a study of thermal explosion theory, (2) an application of it to characterize test methods, and (3) an experimental investigation of the behavior of some thermally unstable compounds using a comercial scanning calorimeter. In this appraisal, definitions of thermal instability and self-reaction hazard have been developed. The importance of thermal explosion theory as a guiding principle in this field is presented. The usefulness of sensitive calorimetric tests is explained. The experimental results of our studies using a scanning calorimetry test method are given and the applicability and limitations of the method are discussed.

This study was sponsored by the Office of Hazardous Materials Operations, Materials Transportation Bureau, Department of Transportation (DOT). That office has supported these studies as a part of its own long range program to improve both the systematization of its regulations for the interstate shipment of hazardous cargoes and the emergency response systems which can be implemented in the event of an accident.

At the present time, thermal instability is not used as a hazard indicator, per se, by DOT, although thermal instability either is or can be implicitly involved in the indicators "explosive," "flammable liquid," flammable solid," or "flammable compressed gas." Thermal instability or self-reaction hazards are currently identified and handled in two of the shipper rules and regulations of Tariff Act 14 (i.e. as of October 1 , 1979, the Hazardous Materials Safety Act, Pubiic Law 93-633). 
The first regulation, section 173.21, prohibits the shipment of any material that can polymerize or decompose under conditions normally incident to transportation so as to cause the dangerous evolution of heat or gas. Such materials can be shipped when properly stabilized or inhibited. The second regulation, section 173.51, forbids the shipment of explosive compositions that ignite spontaneously or undergo marked decomposition when subjected for 48 consecutive hours to a temperature of $75^{\circ} \mathrm{C}$.

Currently, the preceding regulation cannot be unambiguously implemented because there is no consensus agreement either as to the precise definition or test(s) for self-reaction hazards. The absence of a clear definition and unambiguous test procedure increases the probability of failure to identify materials that are currently designated as safe (i.e. under normal shipping conditions) but which can become thermally unstable either under different packaging conditions or when an accident occurs. Since the consequences of a thermal explosion can be as disastrous as the detonation of an explosive, there is a clear mandate to identify these borderline thermally unstable materials and the conditions under which they can become hazards.

Thermal explosion theory is a well developed but complex branch of physical chemistry. Experimental thermal explosion research has been carried out extensively for many years. The applications of this research are many and diffuse -ranging from high explosives to building materials. The literature of this research, is scattered. We have attempted to pull together those parts of thermal explosion work that are pertinent to the thermal instability problem and have used this synthesis in developing our conclusions. Roughly one-third of this report is devoted to this exposition, which appears in appendices originally prepared as 'Norking notes. This treatment may be useful as a starting point for 
A summary of the previous two studies carried out at NBS for DOT is given in Chapter 2 to provide some background for the brief summary of the conclusions and recommendations of the current study that are presented in Chapter 3. Chapter 4 contains a more detailed summary of our current understanding of the implications of the concepts of thermal explosions and tests for self-reaction hazards in terms of tests for and definitions of thermal instability. Chapter 5 consists of a more detailed summary of the experimental work. Detailed conclusions drawn from various parts of the work are presented in sections $4.3,4.4,4.5$ and 5.6.

- Throughout the report there are many references to the appendices where details are developed. But the essential parts of the arguments are given in the body of the text. An attempt has been made to maintain a consistent notation. A glossary of symbols used may be found in Appendix D. The terminology is that used in our previous reports and corresponds to common usage in the literature. The term "thermal explosion" refers to the rapid large rise in the temperature of a material caused by the heat liberated by a run-away self-reaction of the material. However, the term "explosion" is less specific and depends upon the context in which it is used. It can be a detonation, a violent rupture of a container, or a violent fragmentation of a material. "Explosives" refer to materials specifically designed to function by the substantially instantaneous release of gas. and heat.

The authors wish to acknowledge the extensive help they have received from others. E. S. Domalski, G. T. Armstrong and E. J. Prosen, all of NBS, have discussed and illuminated many points. Allen Duswalt, Hercules, Inc., has made suggestions and provided samples. Bruce Cassel, William Brennan, and M. J. O'Neill, Perkin-Elmer Corporation, have helped us with instrumental problems. The exacting task of organizing and typing this report has been done by Miss Darlene Connelly, with precision, perseverance and exceptional good humor. Corrections to the final draft have been made by Mrs. Donna Whitworth with care and patience. 


\section{Summary of Previous Work and Comments}

\subsection{First Appraisal of Methods for Estimating Self-Reaction Hazards}

The initial NBS study [1] for DOT consisted of an appraisal of whether or not some existing empirical criteria for estimating the degree of self-reaction hazard might suffice for evaluation of this hazard potential in a transportation context. The study also sought to determine if the thermochemical and kinetic parameters needed to calculate numerical values for these criteria for the degree of self-reaction hazard could be estimated for materials where no detailed experimental determination of reaction products or reaction rates has been made. The particular criteria and thermochemical estimation procedures involved were those in two predictive schemes: the CHETAH program [2-4] and Stull's adaption of the CRUISE program $[5,6]$. This work has been reviewed by Domalski and Tsang [7]. A more concise summary is given in Appendix A. 3.

The two main results of this initial study were:

(a) That at present self-reaction hazards should be evaluated by experiment rather than solely by prediction schemes. The schemes were judged inadequate for this purpose.

(b) More experimental sensitivity data are needed for borderline hazardous compounds and monomers. Test methods to be used to acquire these data were not specified.

What the methods should be is an important part of the problem (see comments on pages 36,37 and 76 of reference [I]). In particular, there is a need for a more precise definition for self-reaction hazards and an unambiguous procedure, e.g. a coordinated set of tests, for characterizing the hazard. 


\subsection{Second Appraisal of Methods}

In the second NBS study [8] for DOT, the appraisal of predictive schemes started in the first study was completed. Three new tasks were undertaken whose general aim was to determine if a (consensus) definition of thermal instability and corresponding test(s) existed in the hazard evaluation literature and to assemble the information needed either to document or to develop such a definftion and tests. These tasks were: collection and evaluation of test methods and accompanying test data, evaluation of accidental polymerization hazards and a survey and analysis of thermal explosion theory and associated experimental work. A summary of the second study has been published by Domalski [9]. The main results of the second study were as follows:

(a) Predictive Schemes. Efforts to improve the thermodynamic estimation procedures by trying to develop rules to predict decomposition products appropriate to specific reaction mechanisms were unsuccessful. The tendencies of the predictive schemes to overrank the hazard of stable materials and to fail to identify polymerization reaction hazards, as such, were more fully documented. A review of the predictive scheme studies suggested that the ideas needed to develop a clearer definftion of thermal instability had already been identified in the initial survey of thermal ignition theory (see p. 72,73 of [1]).

(b) Thermal Explosion Theory and Experimental Nork. A preliminary evaluation of review articles on theory [10-19] and some associated experimental work (e.g. $[20-24]$ ) supported this conjecture.

(c) Test Yethods. The array of test methods and test data that was collected turned up nothing obvious in terms of a consensus definition or tests for thermal instability. Therefore, methods were analyzed to 
determine whether they measured primarily sensitivity to or power of explosion. It was found that most thermal sensitivity tests could be linked to some aspect of themal explosion theory. A tentative grouping of test methods was made according to their potential relevance in developing a test procedure for evaluation of self-reaction hazard. (d) Polymerization Hazards. Free radical polymerization of olefins, initiated most probably by peroxides, was identified as being the most likely polymerization process to occur inadvertently during transport.

\subsection{Comments}

During the present work the first NBS study has been reanalyzed. This has been done in the light of current information and also to answer comments made by others on the treatment of the CHETAH and CRUISE programs.

First, the CHETAH program is an ongoing project of ASTM Committee E-27. The analysis made in the first NBS study may not be applicable today. A current, experimental version of the program includes a novel method for correlating hazards and thermodynamic parameters. Second, the documentation for the earlier version of CHETAH makes it clear that the predictive scheme was not intended to be a stand-alone method for identifying self-reaction hazards, but is to be used as a first, screening, step in some more elaborate test procedure. Third, while the first NBS study criticizes the empirical criteria of the Chetah program because they are based on parameters more directly related to explosive power than to sensitivity, the criteria do identify shock sensitivity.

These comments indicate that the objections to CHETAH raised in the Eirst NBS study may not be appliable to explosives. However, they may still apply to the screening of borderline thermally sensitive compounds. An appreciable fraction of these borderline compounds may not be shock sensitive. 
The relationship between the CHETAH criteria and explosive power is discussed in Appendix A.1. Earlier conclusions are revised in the light of recent developments. The relationship between the thermodynamic parameters examined by Stull (CRUISE) and the NFPA ratings is discussed in Appendix A.2. 


\section{Summary of Present Work and Conclusions}

Thermal explosion theory has been examined for those features that are applicable to an assessment of thermal instability. These results are presented in terms of the explosion temperature test and tests involving programed heating.

A definition of thermal instability and one for thermal instability hazards are presented that are suitable for use in a transportation context.

The existing hazard test methods have been analyzed in terms of what they measure and their relationship to theoretical concepts. The principles of adiabatic and heat conduction calorimetry are explained insofar as they apply to the present problem.

An experimental study has been made on four thermally unstable compounds using a proposed ASTM test method, which is an application of differential thermal analysis. The usefulness and limitations of this method are discussed. Suggestions are made for extending this method.

The principal conclusions drawn from this work are as follows.

- Both the material and its package must be considered in defining a hazard potential test.

- Thermal explosion theory is well established and is the appropriate formalism for the evaluation of hazard tests and for scaling up laboratory tests.

- Thermal instability can be defined in terms of thermal explosion theory but this is insufficient. A hazard (i.e. potential damage to the environment) must also exist. Thus, we define a thermally unstable material-package configuration to be one: a) composed of substances that decompose or react with evolution of heat, b) for which a thermal mechanism of initiation of a temperature rise in the substances of the package is present (e.g. a slight increase in external temperature), and c) in which a rapid, large increase in the temperature of the substances 
can occur. A thermal instability hazard exists if the decomposition (or reactions) of the material-package configuration results in destruction of the package or neighboring objects or poses a safety threat. The definition of thermal instability is a generalization of the capability of producing a thermal explosion in a material. The definition of a thermally instability hazard incorporates the ideas that a thermal explosion may not be hazardous and that decompositions (or reactions) without a thermal explosion (e.g. evolutions of gases in an unvented container) may be hazardous. These definitions are used to evaluate existing tests.

- Thermal instability tests are only part of the tests required for hazard assessment.

- Some key requirements for thermal instability test(s) are identified. An adequate procedure for determining thermal instability can probably be developed through complementary studies using explosion temperature and calorimetric tests. The tests would have to be carried out with large amounts of sample under conditions that mimic bulk behaviour. These conditions permit scale-up of test results for cases where the formalism of explosion theory is not well developed.

- The experimental results of applying differential scanning calorimetry (DSC) in a proposed ASTM test method partially validate that method. For the materials studied, nitrocellulose, m-azidobenzoic acid and azidotriphenyl methane, our results agree with those obtained by others using quantitative differential thermal analysis (QDTA). One other compound, polyacrylonitrile, gave ambiguous results because of the deposition of products within the instrument. The principal reservations concerning the proposed method are (a) non-applicability to complex systems and (b) large uncertainty in extrapolation of the results to lower temperatures. 
- The principles of operation of DSC and QDTA instruments have been developed. To our knowledge this report is only place they have been analyzed as a group in a compact fashion. As applied to the ASTM method they are formally the same. Methods of extending the data reduction procedures to take into account the extent of decomposition are presented. Experimental modifications are suggested.

- Scanning methods that use very small samples should be compared with heat conduction and adiabatic calorimetry studies on large samples. This should be done to evaluate the limits of applicability of the scanming methods in a hazard assessment procedure. 


\section{Thermal Explosions}

\subsection{Introduction}

Thermal explosion theory and its application to hazard testing are the subject of this chapter. The concepts of the theory are described and experiments that validate it are discussed. The definitions of thermal instability and a thermal instability hazard are developed. This definition and the theory are used to analyze and classify selfreaction hazard tests in order to assess the usefulness of the concepts in practice. Finally, a description is given of several calorimetric rests that are used in (or proposed for) hazard evaluation. These are state-of-the-art tests that require the use of theory in the application of their outputs to hazard assessment.

These topics are covered in sumary form with emphasis on key concepts and conclusions. More detailed discussions are given in appendicies, with appropriate cross references here.

The organization of the chapter is as follows:

(1) Models of two types of explosion tests are introduced in Section 4.2. The characteristic phenomena of thermal explosions are described. These models are used in the detailed analyses in later sections.

(2) Section 4.3 presents the basic physical ideas of thermal explosion theory, the approximations that are commonly used, and our conclusions. It is based on a systematic treatment of those parts of theory applicable to the present problem. That treatment was developed because the principal reviews of the field [10-22] neither present the theory in a concise form easily applicable to the present problem nor use a common notation. Details are given in the first eight sections of Appendix 3. 
(3) Section 4.4 contains an analysis of experiments that validate thermal explosion theory. It also characterizes practical hazard tests in terms of theoretical concepts and what they measure. General conclusions are given. More details appear in the last three sections of Appendix B.

(4) Section 4.5 is a description of four types of calorimetric sests. What is measured is explained together with the necessary theoretical treatment of the data. The section is background for chapter 5 on our experimental studies. The material treated in section 4.5 is presented in detail in Appendix C. 


\subsection{Models For Thermal Explosions}

The physical principles underlying the mathematical theory of thermal explosions are simple and beyond question. Heat is generated in a source (the decomposing sample) held in a container and this heat then flows to the surroundings (the environment) which are held at a fixed temperature. The rate of heat generation increases exponentially with temperature, a common characteristic of chemical reactions, while the heat flux (i.e. heat transfer rate) between the sample and its environment is approximately a linear function of their temperature difference. If the rate of heat generation can exceed the heat flux, a thermal runaway or explosion may result.

The problems that arise in the mathematical treatment concern specification of the behavior of the heat source, the heat transfer within the sample and the heat flux to the environment. The rate of heat generation depends upon the kinetics and mechanism of the chemical reaction as well as on its exothermicity. The mode of heat transfer in the sample may be conduction, convection, or a mixture of these. The heat flux to the environment, if solely by conduction, depends (as does heat transfer within the sample) on temperature gradients which are controlled by thermal conductivities and heat capacities.

For various boundary conditions the behavior of a sample will differ. The equations describing the temperature of a sample as a function of time are non-linear and can only be solved numerically for the general case. Analytical solutions for limiting cases are developed using various approximations. These must be justified for each class of experiments. 
But the approximate, analytical solution remains very important.

It provides a qualitative guide to the phenomena revealed in detail by numerical calculations, it is the basis for classifying test methods, and it provides guides for scaling up laboratory experiments to shipping and storage conditions.

The exposition in the next section is developed with reference to two idealized experiments, one of the explosion temperature test and the other of programmed heating test of a material. These are described below and used to introduce the principal terms used later. In the explosion temperature test, the decomposing material is assumed to be homogenous. Thus, some details of the explosion temperature test, such as the location of the initiation of an explosion, will not necessarily apply to surface oxidation reactions.

\subsubsection{Explosion Temperature Test}

In this test an encapsulated sample, originally at room temperature, is immersed in a bath at a series of fixed, higher temperatures. The samples either explode or they do not. The limiting or critical temperature of the bath dividing the explosive and non-explosive samples is sought. The time to explosion increases exponentially as the positive difference of the actual minus critical bath temperature approaches zero. The critical bath temperature depends on the size and geometry of the sample and the physical parameters mentioned earlier.

In more abstract terms, and with some attention to the temperature history of the sample, this test can be described as follows. A sample and its environment are originally at a low, spatially uniform temperature, $T_{\text {in }}$ At zero time, the temperature of the environment is stepjumped (raised at an infinite rate) to a fixed high temperature, $T_{0}$. The sample temperature, $T$, rises as heat flows in. Depending upon the value of $T_{0}$ several things may happen. 
When $T_{0}$ is moderate and the sample is essentially inert, $T$ rises to $T_{0}$ and remains there. If $T_{0}$ is such that it causes a slight generation of heat in the sample, $T$ rises rapidly to $T_{0}$ and then, much more slowly, to a slightly higher temperature at which heat generation and flux are balanced. The maximum temperature is at the center of the sample. As the sample reacts the rate of heat generation decreases and, eventually, T declines toward $I_{0}$.

The critical temperature of the environment, $T_{*}$, is the highest for which a balance between heat generation and loss can occur.

When $T_{0}$ is slightly higher than $T_{*}$, the temperature of the sample rises quickly to $T_{0}$, rises more slowly toward the critical maximum for stability and then continues upward at a sharply increased rate. This is the thermal sunaway and is moderated only by exhaustion of the sample. The maximum temperature at any time (when $I>T_{0}$ ) is still at the center of the sample. (This picture is also used in the treatment of explosions due to "hot spots".)

When $I_{0}$ is substantially higher than critical, the outer portions of the sample exceed critical conditions before the center warms up to $T_{0^{\circ}}$ Explosions start in an annulus that approaches the edge as $T_{0}$ is raised. This is the "ignition regime" which is of importance for explosives but is of less interest for borderline hazardous materials (since they are not explosives or detonators) or for setting criteria for handing.

The model of the explosion temperature test is applicable not only to the interpretation of laboratory experiments but also to accident conditions involving fires and adiabatic compression. It is described again in Appendix B.I, developed in a simplified manner in 3.2 to 3.5 , and extended to complicated cases in 3.6 and 3.7 . 


\subsubsection{Programmed Heating Test}

The second test is used to examine the thermal decomposition of a material when the temperature of its environment increases linearly with time, $T_{0}=T_{i n}+w t$. ( $w$ is the rate of increase in the environment temperature and $t$ is the time.) This is the analogue of the ASTM procedure discussed in chapter 5 and also applies to diurnal heating of materials in storage. The model is developed in detail in Appendix B.8.

Briefly, as $T_{0}$ is raised at a fixed rate, the temperature of the sample follows it (with a small lag) as heat flows in. At some temperature the heat generation becomes appreciable and T rises above $T_{0}$, passes through a maximum and then, as the reaction is completed, decays back to the (still rising) value of $T_{0}$. In a properly designed experiment there is no thermal runaway.

Chemical rate constants can be derived from a study of the departure of $T_{\text {from }} T_{0}$. The simplest method correlates the maximum temperature, $T_{d}$, with the programmed scanning rate, 'w. The enthalpy of reaction can also be obtained. Detailed analysis of the exotherm can provide data on the kinetic rate law.

Critical temperatures are not measured in this test. Instead the rate constants and enthalpies, together with heat capacities and thermal conductivities are used to predict the critical environment temperature of the material under other conditions. The scaling rules used in this process are based on thermal explosion theory. 


\subsection{Thermal Explosion Theory}

The goal of the exposition here is to define thermal instability within the context of thermal explosion theory. Validation of the definition for use with experiments is covered in a later section. In order to develop this definition and to provide a framework for understanding and assessing experiments of the previous section, a review of the main points of theory is necessary. It is given in short form here and in detail in Appendix $B$.

\subsubsection{The Isothermal (Uniform Temperature) Sample}

This case, the simplest to treat, assumes that the temperature within the sample is spatially uniform. It corresponds to highly efficient convective mixing or to a stirred liquid. Essentially all descriptions of more complex cases can be developed in terms of (small) extensions of this case if attention is restricted to the temperature region near the critical point. Assumptions are given in Appendix B.2.

The heat balance for the entire material is

$$
C(d T / d t)=Q^{\prime}(d n / d t)-h^{\prime}\left(T-T_{0}\right)
$$

where $C$ is the total heat capacity of the naterial (excluding the container), $Q^{\prime}$ the total heat liberated by the reaction, $n$, the fraction of the sample reacted, and $h^{\prime}$ is an empirical average heat transier coefificient between the sample and its container. $I_{0}$ is the temperature of the sample container which is assumed to be constant and equal to that of the environment of the container.

In general the rate of decomposition (or reaction) of the sample is given by the kinetic equation

$$
\mathrm{d} n / \mathrm{d} t=f(\eta) k(T)
$$

where $f(n)$ is an arbitrary but often simple function of the fraction decomposed and $k(T)$ is the rate constant. For many cases the rate may be 
considered independent of concentration and $f(n)$ held constant at a value near its maximum, $f(n)_{m}^{+}$, for the temperature range in question, yielding a commonly used form

$$
d n / d t=f(n) \cdot k(T)
$$

This approximation is most appropriate when $Q^{\prime}$ is high and the rate increases very rapidly with temperature: an explosion occurs before appreciable decomposition occurs, or while $\eta$ is changing slowly.

The usual assumption made for the rate constant is that it has the Arrhenius form

$$
k(T)=A \exp (-E / R T)
$$

although this is not necessary. Here $A, E$ and $R$ are the preexponential factor (dimensions of $t_{i m e^{-1}}$ ), the activation energy and the universal gas constant.

Analytical treatments recast equation (1) into dimensionless form and introduce three parameters, $\delta^{\prime}, B i$ and $B$ or a related parameter, $B_{*}$, which will be used throughout this discussion.

The first, $\delta$, is the rat1o of the rates of change with temperature of the heat generation and heat loss rates on the right hand side of eq(I) evaluated at $\mathrm{T}_{0}$.

$$
\delta^{\prime}=\left(Q^{\prime} / h^{\prime}\right)\left(E / R T_{0}^{2}\right) f(\eta)_{m} \exp \left(-E / R T_{0}\right)
$$

A thermal explosion, for this model, can occur only if $\delta^{\prime} \cdot>1 / e$, e $=2.718 \ldots)$. When $\delta^{\prime} \leq 1 / \mathrm{e}, \mathrm{T}-\mathrm{T}_{0} \leq \mathrm{RT}_{0}^{2} / \mathrm{E}$. Since $\mathrm{RT}_{0} / \mathrm{E}$ is usuaIly 0.001 to 0.01 , the critical temperature rise is small.

In the working notes of the appendices the symbol $f\left(\eta_{m}\right)$ is used in place of $f(\eta)_{m^{*}} f\left(\eta_{m}\right)$ is mathematically incorrect. 
The crucial importance of $\delta^{\prime}$ is that it is a measure of sensitivity to explosion and that its values near the critical temperature can be predicted. This can be done for specific heat transfer condttions using simple steady state heat transfer theory. An example is given in the development of eq. B-31 and agreement with exact solutions is shown to be about 10 per cent. This approach works because, just prior to explosion, the materlal is almost in a steady state ( $\mathrm{dT} / \mathrm{dt}$ is almost zero).

The second parameter, $B i$, is the Biot number for heat transfer. It compares the efficiency of heat transfer between sample and environment to. that by conduction within the sample. In general it is defined as

$$
B i=\alpha_{0} \ell / \lambda
$$

where $\alpha_{0}$ is the overall heat transfer coefficient per unit area of contact between sample and environment, 2 is a characteristic length, and $\lambda$ is the thermal conductivity within the sample. For a sphere 2 is the radius. Small values of Bi.correspond to efficient conductive heat transfer and, hence, nearly uniform temperatures within the sample. As Bi increases, thermal gradients build up within the sample.

The third parameter, $B$, is called the dimensionless adiabatic temperature rise

$$
B=\left(Q^{\prime} / C\right)\left(E / R T_{0}^{2}\right)
$$

$B$ is a measure of the total rise in $I$ (for the sample) above $I_{0}$ in the explosion temperature test when $h^{\prime}$ is set equal to zero in eq. I (after I has risen to $\left.T_{0}\right)$. More exactly, $B$ is the maximum value of the dimensionless temperature rise, $\theta$, where

$$
\theta=E\left(T-T_{0}\right) / R T_{0}^{2}
$$

The related parameter, $B_{*}$, is defined as

$$
B_{\frac{1}{*}}=\left(Q^{\prime} / C\right)\left(E / R T_{*}^{2}\right)
$$


where $T_{*}$ is the value of $T_{0}$ when $\delta^{\prime}=1 / e^{\prime}$, the critical value of $\delta^{\prime}$ in eq. 4. The criterion for a discernable thermal explosion in the explosion temperature test is stated to be that the value of $B_{*}$ should be greater than a number ranging from 5 to 100, depending upon the Iiterature source. (The preceding statement is ordinarily stated using $B$. Since $T_{0}$ must be greater than $T_{*}$ for a thermal explosion to occur and $B_{*}$ is the maximum value of $B$ under explosive conditions, $B_{*}$ rather than $B$ is actually meant.) $B_{\star}$ is a measure of the power of an explosion.

We now return to the explosion test described in 4.2. Analysis of the temperature-time and fraction-decomposed-time relations (Appendix B.4) shows that the isothermal sample model based on eq. $2 b$ approximates more realistic cases provided the change in $f(n)$ is small prior to thermal explosion (e.g. for explosives) and provided $\delta$ is only slightly larger than 1/e. The warmup time (that in which $T$ rises from $T_{i n}$ to $T_{0}$ ) when $\delta^{\prime}$ is close to $1 / \mathrm{e}$ is short compared to the "induction time" (that time in which $T$ rises from $T_{0}$ to $T_{0}+2 R T_{0}^{2} / E$. $T_{0}+2 R T_{0}^{2} / E$ is a rough measure of the temperature of the sample at the onset of an explosion). The induction times become shorter as $\delta^{\prime}$ (and $T_{0}$ ) increase but under most conditions the warmup time is insensitive to $\delta^{\prime}$. The fraction of the sample decomposed prior to explosion decreases as $B_{*}$ increases. When the "ignition regime" is reached, $\delta$ ' $>1 / e$, the warmup period is no longer short compared to the induction time, due mainly to the decrease in the latter. One result of this interplay of variables is that a plot of the logarithim of explosion time versus $1 / T_{0}$ is not linear and its slope is not a good measure of the activation energy.

\subsubsection{Criterla for Explosion}

The analysis has been extended to the more general isothermal sample kinetic equation ( $2 a)$, that is with $f(n)$ a variable. This allows for 
depletion of reactant. Here the sharp distinction between explosive and non-explosive states disappears. Only numerical solutions are appropriate. Details are given in Appendix 3.5. Four types of explosion criterla were examined for these more realistic cases and are the basis for later conclusions. They are:

a) The value of $T_{0}$ for which a one percent change in the parameters that characterize the critical condition (mainly $\delta^{\prime}$ ) can cause a doubling in the maximum temperature that the system can attain.

b) An inflection point in the curve of temperature versus fraction. of material decomposed; that is, onset of upwards curvature in $T$ when $T$ is greater than $T_{0}$.

c) An inflection point in the temperature-time curve.

d) High sensitivity of the temperature-time or temperaturefraction material decomposed curves to small changes in $I_{0}$ or $h^{\prime}$. This is the mathematical analogue to the experimental irreproducibility found in replicate experiments when $T_{0}$ is near its critical value.

For simple reactions with

$$
f(n)=(1-n)^{n}
$$

where $\mathrm{n}$ is a positive integer, and with $B_{*}$ greater than ten, all of these conditions yield results that indicate that the critical value of $\delta$ ' has the value $1 / \operatorname{ef}\left(\eta_{c}\right) . \quad f\left(\eta_{c}\right)$ is a constant for a particular experiment.

$$
f\left(n_{c}\right) \sim f(n)_{m}\left[a_{1}-a_{2}\left(n / B_{*}\right)^{2 / 3}\right]
$$

In this expression $f(\eta)_{m}$ is the maximum value of $f(\eta) \cdot a_{1}$ and $a_{2}$ are weak functions of $B_{*}$ and can be approximated with constant values of $I$ and 2.4 , respectively. $B_{*}$ is given by eq. $6 b$ where $T_{*}$ is the value of $T_{0}$ when $\hat{o}^{\prime}=(1 / e) f\left(\eta_{c}\right)$. Where thermal explosion phenomena are easily discernable, criteria a) through c) give essentially the same critical 
conditions for a first order reaction while criterion d) classes the material as more hazardous. When thermal explosion phenomena become less discernable $\left(1 / B_{*} \sim 0.05\right.$ to 0.1$)$, the predictions of the range of the non-explosive states for a first order reaction diverge rapidly (for reasons discussed in Appendix B.5.). Criterion a) says that if $B \leq \sim 20$ all states are non-explosive, while, for criterion b), this limit is $B_{*}$ 5 4. Criteria c) and d) say explosive states occur well past $B_{*} \sim 4$; the range of non explosive states being larger for criteriond) than c). (The predictions of the criteria also diverge for higher order reactions even when explosions are eastly discernable.)

These results mean that a single criterlon, however arbitrary, must be adopted for the comparison of theoretical treatments and for application to experiments.

\subsubsection{Extension to Non-Isothermal Samples and Complex Kinetics}

Heat transfer within the sample may be by conduction or by convection (forced or free) or a mixture of these. The temperature profiles will be different and are very difficult to analyze, especially when the sample-geometry is irregular. The isothermal model is the limiting approximation of these complex cases in which the temperature gradients are small. If heat transfer is by conduction, the gradients are small provided that the Biot number, eq. 5, is small. If this condition is satisfied, then the isothermal model can be used up to the time a thermal explosion does occur ${ }^{+}$. The isothermal model can apply with reasonable accuracy throughout the course of the entire decomposition if heat transfer conditions, are arranged so that a thermal explosion never occurs or if $B_{*}$ is very small.

\footnotetext{
tI is still assumed the reaction kinetics are simple and homogenous and that the environment boundary condition is a constant, uniform ambient temperature. References to other boundary conditions are given in Appendix B. 6 .
} 
The expression for the critical parameter, $\delta$ ', of non-isothermal but physically homogeneous samples can be written in the form of eq. 4 when heat transfer within the sample is solely by conduction and the environment temperature is uniform. For example, for a sphere one obtains eq. (4) if $Q^{\prime} / h^{\prime}$ is given by

$$
\frac{Q^{\prime}}{h^{\prime}}=\frac{Q^{2}}{\lambda^{2}}(0.101)\left(\frac{3.29}{B i}+1\right)
$$

$Q$ is the heat generated per unit volume, $\lambda$ is the thermal conductivity, and $r$ is the radius of the sample. (The numerical values of 0.101 and 3.29 as well as the functional dependence on $B I$ are those predicted by the extension of the 1sothermal model outlined in Appendix B.3. The values of $\delta^{\prime}$ predicted by eqs. 4 and 10 agree with those of exact treatments within 10\%). Equation 10 or analogous equations for other simple geometries and eq. 4 can be used to predict the conditions for thermal explosions from laboratory test results. For this reason, eq. 4 can be viewed as a scaling relation.

The chemical reaction may be autocatalytic (promoted by products), occur in two phases (heterogeneous), or have a multiple step mechanism. It has been shown by others that there are relatively straightforward mathematical techniques for extending the analysis for critical conditions of a single reaction to these cases using the isothermal model provided we approximate the various $f(\eta)$ as constants. The literature on this topic is reviewed in Appendix B.7.

\subsubsection{Programmed Heating of Unstable Materials}

This model has been described in section 4.2 and is discussed in Appendix 3.8. Only the case of linear heating of the environment is considered here although alternatives have been suggested in the fields 
of thermogravimetric and differential thermal analysis. The rising temperature of the environment forces a similar rise in temperature in the sample. There is a critical value of the heating rate, $w_{*}$, that is analogous to $T_{*}$, the critical environmental temperature in the step-jump case. But the temperature of the environment at which the maximum rate of decomposition of the sample occurs (a peak on a temperature-time plot) is greater than the critical explosion temperature; $T_{*}$, of the stepjump case. This different behavior is due to the increased importance of extent of reaction. At low heating rates more material can decompose in a given temperature interval than at high heating rates. Numerical values of this critical heating rate can apparently be predicted to within the order of fifty percent or so by an approximate analytical theory based on the assumption the material is always in the quasisteady state (i.e. $d T / d t=w$ ) if $w$ is less than the critical value.

\section{3 .5 Conclusions}

(1) While the consequences of a thermal explosion may be different for a material that is an explosive by design (e.g. TNT) and one which is only capable of mild burning, the conditions for a thermal explosion to occur are the same. The conditions are dependent upon the heat evolution rate of the material as a function of temperature, the heat transfer characteristics of the material, its container and container surroundings, and the fraction of the material that has decomposed. Thus, statements to the effect that a material is thermally unstable or thermally hazardous, while common (in hazard test literature) and convenient, are incomplete. The amount of material and packaging conditions are an equally important consideration and can completely alter whether a thermal explosion can occur for a given ambient temperature. It is also relevant to note that 
the conditions that determine whether a thermal explosion occurs depend upon the heat evolution rate as a function of the fraction of material decomposed and temperature just prior to the explosion. Specific or detailed mechanisms of decompositions are not, per se, relevant except as they affect heat evolution rates.

(2) Statements to the effect that a discernible thermal explosion does not occur if the heat liberated by a single exothermic Ieaction, more exactly the parameter $B_{*}$ of eq. $6 b$, is below a certain range of values are theoretically meaningless unless the criterion for a thermal explosion upon which the statement is based is also specified. Practically, it appears that the statement is true if $B_{*}$ is less than 5 to 10. Statements to the effect that there are no critical conditions at all if the rate of decomposition of the initial material depends upon the fraction of material decomposed are misleading.

(3) The sharp simple mathematical distinction between an explosive and a non-explosive state of a material occurs only in the approximation that the rate of decomposition of the material is independent of the amount of material decomposed, (i.e. the zero order reaction). The simple distinction is lost when the variation of the rate of decomposition with fraction of the sample that has decomposed is taken into account. This loss does not mean, however, that critical conditions based on the zero order approximation are invalid but, rather, that the conditions must be modified. The investigations examined in the study indicate that the modification of the zero order critical values of the parameters that dictate whether or not an explosion occurs is simply to multiply them by a factor that takes into account reactant consumption. For reactions in which $\mathrm{n}$ is a positive integer in eq. 8, this factor difiers negligibly from one, for our purposes, if $B_{*}$ is large (i.e. greater than 100) since 
$f(n)_{\text {m }}$ equals one. For simple reactions, then, it seems reasonable, for our purposes, to approximate the critical value of $\delta^{\prime}$ as $1 /\left(\mathrm{ef}(n)_{\mathrm{m}}\right)$ unless the material is only weakly unstable.

(4) Identification of thermal instability. It is our conjecture that further theoretical and experimental studies of the stability criteria studies of single reactions, will show that the critical conditions for a thermal explosion are close to.those of explosion criterion b), listed in section 4.3.2. It is our proposal that criterion c), an inflection point in the temperature time plot when $T>T_{*}$, be tentatively adopted as the analytical condition for identifying thermal explosions until it can be established that the less conservative criterion, b), an inflection point in the temperature-reacted fraction plot, is a more realistic condition (i.e. in practice).

(5) It seems reasonable on physical grounds to conclude that if a material decomposes by a sequential set of decomposition reactions, the reaction which has the lowest critical temperature (i.e. Q', E, h' for each reaction in the sequence would be different) is the trigger which determines whether or not a thermal explosion takes place. ${ }_{*}$ for the "trigger reaction" may not determine whether the thermal explosion is discernable. For example, $B_{\star}$ for the trigger reaction may be small so that only a minimal amount of self heating occurs. If, however, the liberated heat is sufficient to raise the temperature of the sample above $T_{*}$ for another reaction for which $B_{*}$ is large, a vigorous explosion may occur.

(6) Suppose one rates the degree of thermal instability of a given material-packaging arrangement by the magnitude of the value of $T_{*}$ (i.e. the ambient temperature, $T_{0}$, when $\delta^{\prime}$ equals $\left.1 / e\right)$. Then the degree of instability will be more sensitive to changes in $E$ than $Q^{\prime}, h^{\prime}$, or A. 
$I_{*}$ is nearly linearily proportional to $E / R$ but varies only as the inverse of the sum of the logarithms of $Q^{\prime}, h^{\prime}$, or $A^{+}$For the same reason, the thermal instability would be relatively insensitive to variations in $B_{*}$. Major changes in the degree of instability of a given material are possible only through reducing the thermal relaxation time of the material by either reducing the amount of material or improving heat transfer conditions for each package. Qualitatively, $T_{*}$ may be viewed as a sensitivity criterion for a thermal explosion and $B_{*}$ of eq. (6b) as a part of the factor that evaluates the damage potential of a thermal explosion. The above comments mean that the sensitivity and damage potential, as measured by $B_{\star}$ of eq. $6 a$, are not closely correlated.

(7) The time to explosion in either the step jump or linear heating cases is not a measure of the sensitivity or power of a thermal explosion. For materials decomposing by a single reaction, the time to explosion may be very long if the decomposition is by an autocatalytic mechanism. Yet, $T_{*}$ may be $10 w$ and $B_{*}$ (the dimensionless adiabatic temperature rise) large which makes this material dangerous from both a sensitivity and power point of view. However, under accident conditions the time to explosion can be viewed as a sensitivity criterion for a thermal explosion if $\delta$ 'exceeds its critical value in the sense that some fraction of this explosion time is the time that is available to take emergency response action.

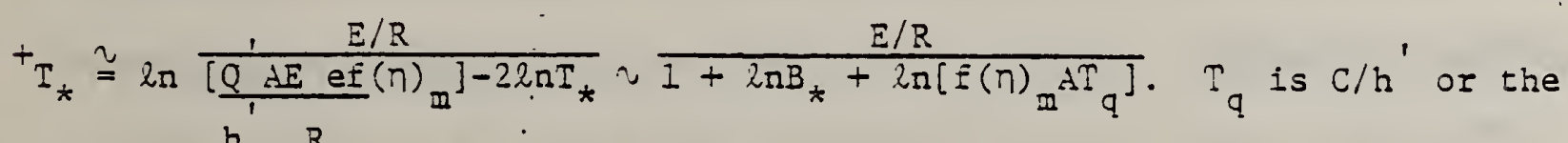
thermal relaxation time of the material. This formula accounts for the better correlation of $T_{*}$ with $E$ than with $Q^{\prime}$ ooserved in the first NBS study for DOT. 


\subsubsection{Definition of Thermal Instability and a Thermal Hazard Criterion}

It seems clear that thermal instability in a transportation context can be defined as the capability of producing a themal explosion. However, this is too restrictive because the term thermal explosion applies only to a limited group of self-reactions. (It applies only to materials whose composition and rate of evolution of heat would be spatially uniform through the course of a hypothetical experiment during which the material is kept uniform during its self-reaction.) The remaining group of reactions, heterogeneous reactions in inhomogeneous materials must be considered since those that evolve heat show the same general type of critical phenomena described in the explosion temperature test, and reactions of this type (e.g. surface air oxidations) are common.

Also, the definition of thermal instability must take into account the fact that any parameter which can alter the heat balance anywhere in or on a package will have a critical value above (or below) which a large rapid increase in the temperature of the package contents can result. Besides the parameter we have discussed, ambient temperature, there are others pertinent to the transportation situation such as radiant heating of a package, or the insulation (i.e. heat transfer coefficient) between the contents and surroundings of a package. Other modes of initiation of ...... decomposition would be local heating by shock or impact which apply to more unstable materials.

To cover these various cases we propose the following definition of thermal instability. A thermally unstable material package configuration is one

(1) composed of substances that can decompose or react with the evolution of heat,

(2) For which a thermal mechanism of initiation of a temperature rise is present (e.g. a slight increase in external temperature), and 
(3) in which a rapid, large increase in the temperature of a material can occur.

For the purposes of defining a test for thermal instability, we propose that condition (2) be modified to:

(2) for which a slight increase in a uniform external temperature can initiate a decomposition or reaction.

Evaluation of the thermal hazard posed by a package involves additional considerations. A material can decompose exothermally and "explode" without a thermal explosion, as defined in section 4.2, having occurred. For example, consider a material in a closed container that decomposes with the liberation of heat and gases by a simple autocatalytic mechanism. A thermal explosion does not occur until the fraction of the material that has decomposed, $\Pi$, is near $1 / 2$. The hot gases could have ruptured the container long before this value of $\eta$ is reached.

It is also possible for a thermal explosion to occur and yet the hazard be small. An example might be a case where the decomposing marerial liberates no gaseous products, the adiabatic temperature rise of the material caused by the decomposition is small enough that little vaporization occurs, and no other decomposition reactions are triggered in the material. The hazard of a spilled hot decomposing material of this type might be dictated solely by cargo compatibility considerations. On the basis of these considerations, it seems reasonable to state the following:

a thermal instability hazard exists for a specific naterial-package configuration at a given ambient temperature if a slight increase in the temperature will cause the material to decompose and result in destruction of the container or objects in the vicinity of the package. 
The lower the minimum ambient temperature for which a hazard exists, the more hazardous the material package configuration. In the absence of other conditions this minimum ambient temperature will be the critical temperature for a thermal explosion.

These definitions are proposed for three reasons. First, it covers the entire spectrum of materials loosely described as being thermally unstable. It applies to explosives, near explosives (e.g. organic peroxides), rocket propellants, monomer with insufficient inhibitor, and cellulosic materials which can undergo spontaneous combustion. (From a practical point of view many materials in these classes are not hazards as shipped. Wood is an obvious example. Also, explosives that may be shipped that are thermally stable at ambient temperature but may become unstable at elevated temperatures.) Second, the definition is consistent with existing safety technology in storage and handling of explosives and other energetic compounds [21, 24]. The third reason is that the physical parameters needed to calculate the critical temperature in practical situations are, in principle, identifiable. Also experimental procedures to measure these parameters in small scale tests have been proposed. Experimene with one such test is summarized in Chapter 5. 
4.4 Thermal Explosion Experimentation and Self-Reaction Hazard Tests

A tentative definition of thermal instability has been given in section 4.3.6. It would be identified as an inflection point in the temperature time curve followed by a rapid rise in temperature of a material (see section 4.3.5). The thermal instability hazard of a material-package combination is also identified: the minimum uniform ambient temperature that will cause the material to decompose and destroy its container or objects in its vicinity. The latter definition implies that either the temperature or pressure rise within the container during the hazardous event.

Since these definitions rest upon thermal explosion theory, it is advisable to test them against experimental work and to examine their usefulness in terms of current self-reaction hazard testing. To do this we proposed the following three sets of questions given below. Answers are discussed in the remaining parts of this section.

(I) In what respects have the various aspects of thermal explosion theory been verified experimentally? Have the problems associated with heterogeneous reactions in solids or the heat transfer problems in real decomposing liquids (free convection or stirring by gas bubbles, erc.) been investigated experimentally?

(2) What is the current state of the art of self-reaction hazard testing and is the proposed definition of themal instability consistent with this practice? If so, what other parameters or characteristics or a material or material-package arrangement are used to characterize the self-reaction hazard besides sensitivity to thermal explosion? What constitutes a complete test for thermal instability if the proposed definition is correct. 
The third question is based on our experimental work, reported in chapter 5 .

(3) In differential scanning calorimetry on thermally unstable materials, can the errors caused by temperature gradients in the sample be reduced by a radical change in experimental conditions? Can this be done without destroying the usefulness of the method? An error analysis based on the theory summarized in section 4.3 suggested that an improvement would result if the effective thermal contact between the sample and its container was kept large while the thermal contact between the container and its environment was kept small. What are the merits and Iimitations for this plan and what evidence for it is in the literature?

\subsubsection{Experiments on Explosions in Gases}

A limited number of experimental articles on gaseous systems were reviewed and are sumarized in Appendix B.9. In brief, the articles indicate that the predictions of thermal explosion theory have been confirmed for simple gaseous systems that decompose by a single reaction. Rayleigh numbers must be less than 600 for purely conductive explosion theory to apply. Measured critical temperature distributions and calculated values of $\delta_{c}$ (i.e. using eq. B-3l with experimental values for the various parameters and the critical value of the ambient temperature) agree with those predicted by theory (i.e. in the case of $\delta_{c}$; the value given by eq. $B-30$ for infinite $B i$ and 3.32 for $g_{I} / g_{2}$ for the sphere). Values of the energy of activation determined from critical pressuretemperature measurements agree with values determined by other techniques. An explanation has been given for the failure of the measured critical pressure to vary as the nth root of the thermal conductivity of the gaseous reactants, where $\mathrm{n}$ is the order of the reaction, that had been 
observed in many earlier investigations. More recent work has been aimed at developing a stirred-flow reactor so that thermal explosion studies can be carried out under conditions for which the isothermal model applies.

\subsubsection{Experiments on Explosions in Condensed Phases}

A limited number of articles reporting experimental work in the USSR on condensed phase explosives were reviewed (see Appendix B.10.) These articles were selected because they amplified a review article on experimental work by Merzhanov [22] and cover a time span up to 1970. They describe a sequence of experiments that clearly is aimed at establishing experimental conditions under which the isothermal model mentioned in section 4.3 can be applied not only to the measurement of critical conditions for thermal explosions but also to the rate of heat evolution during the entire course of a decomposition reaction. The compounds most often studied were two explosives, DINA (dinitroxydiethylnitramine) and tetryl (N-methyl-N, 2,4,6 tetranitroaniline). Both decompose rapidly or thermally explode only in the liquid state. A brief sumary of the sequence of experiments is as follows.

(a) DINA and tetryl both decompose by a single reaction provided gaseous products are allowed to escape (i.e. to prevent catalysis or other reactions by products). DINA decomposes by first order and tetryl by simple autocatalytic kinetics. Kinetic parameters obtained by isothermal (i.e. constant temperature) differential calorimetry and weight loss measurements (i.e. thermogravimetric analysis) agreed within their combined uncertainties.

(b) Proof that thermal explosion theory applies to these compounds was developed as follows. Experiments were first carried out on large 
quantities of either DINA or tetryl (200-300 grams) under stirred liquid conditions such that the (measured) temperature gradient in the liquid is negligible. Measured and calculated values of the critical ambient temperature, $T_{*}$, the Semenov warmup of the material (temperature rise of the material above the critical ambient temperature prior to explosion, $\mathrm{RT}_{*}{ }^{2} / \mathrm{E}$ ) and induction times to explosion (calculated by numerical integration) agreed within their uncertainties. The importance of including the approximate effect of the changes in the volume of the decomposing material as they effect $h^{\prime}$ and $Q^{\prime}$ in the above calculations and measurements was demonstrated.

(c) The conditions for the isothermal model to apply to explosion measurements on unstirred liquids was shown to be that the effective Biot number (eq. 5) must be equal to or less than one. Under this condition, for example, a maximum temperature difference of $1 \mathrm{~K}$ within a one gram sample was observed for a $10 \mathrm{~K}$ Semenov warmup. Also, critical explosion temperatures for a one gram sample were kept near those observed in stirred 200 gram samples by keeping $\alpha_{0}$, the overall heat transfer coefficient of eq. 5, small (i.e. order of $10^{-4} \mathrm{~W} \cdot \mathrm{K} \cdot \mathrm{cm}^{-2}$ in the design of the sample container-thermostat apparati. The thermal instabilities of explosives were ranked by direct comparison of explosion temperature measured in identical containers ${ }^{+}$(disposable glass Dewars in this case).

(d) The design conditions outlined in (c) are necessary conditions for the isothermal model to apply to the entire course of the decomposition in isothermal and scanning (i.e. linear heating) differential thermal

\footnotetext{
'In this way $h^{\prime}$ of eq. I is the same for all materials. Since $h^{\prime}$ is small, the behaviour of a large amount of material is simulated.
} 
analysis measurements. Degeneration of a thermal explosion (i.e. reduction of $B_{*}$ defined in eq. $\left.6 b\right)$ can be accomplished by dilution of the reactants with a material of large thermal conductivity and thermal diffusivity. This permits isothermal kinetic measurements to be made above the critical explosion temperature of the material with minimal temperature gradients in the material. An alternative procedure (which is currently believed to be less effective) that can be adopted if $\alpha_{0}$ is to be kept small is the use of a metalic container of large heat capacity placed in good thermal contact with the material. $\alpha_{0}$ then is the heat transfer coefficient between a unit area of the container and thermostat. One is, in effect, then including both the heat capacity of the container and sample in $C$ in the definition of $B_{*}$ in eq. $6 \mathrm{~b}$. Dilution alters $C$ of the material directly. This work confirmed our idea (cited earlier in this section) about how to reduce errors caused by temperature gradients in the sample when using heat conduction calorimeters.

(e) Some studies provided information about complex chemical reactions. These include studies on autocatalysis by decomposition products, heterogeneous reactions, modification of the rate of heat evolution as a function of temperature due to the rate of heat evolution caused by temperature due to vaporization of reactant in product gas bubbles, the effect of free convection on the critical parameters $j c$ (i.e. studies at large Rayleigh numbers), and the combined effect of product gas bubble evolution and free convection on the critical parameter, $\dot{o}^{\prime} c^{\cdot}$. These studies can all be interpreted within the formalism of thermal explosion theory. However they all introduce new extensions of the art and suggest that additional details will be revealed by Euture experiments. 


\subsubsection{Conclusions Concerning Thermal Explosion Experimentation}

(1) Recent experiments on thermal explosions justify the use of theory as a design parameter and a tool for predicting thermal instability.

(2) Studies specifically designed to determine the predictions of thermal explosion theory have been carried out on unstirred gaseous and stirred and unstirred liquid (i.e. at the onset of thermal explosion) reactants. Experiment and theory agree where the reaction is simple and known. Differences probably are attributable to complexities in reaction mechanism or experimental apparati that have not been taken into account (e.g. the former is our view of ref. [93]). Fewer studies of the type mentioned above that deal with solids, which necessarily involve heterogeneous reactions, have been analyzed. Intuitively, it seems clear, however, that any possible divergence between theory and experiment is attributable again to complexity.

(3) The specific form of the effect of free convection on the critical parameters, $\delta^{\prime}$, can now be calculated if the Rayleigh number is known (i.e. Appendix B.10, ref. [114]). (Thus, for 1iquids, in which the effects of gas evolution can be neglected, and for gases, the scaling relation of a fundamental is known if decomposition occurs by a simple machanism.) The form of the effect of bubble evolution on the critical parameters for a decomposing liquid is known at least in part (i.e. Appendix B.10, ref. [113]) and the nature of the "competition" between bubble evolution and free convection (i.e. Appendix B.10, ref. [115]) is known. Variation of the critical parameter seems to be specific for each liquid system. The effect of' a complex reaction involving a simultaneous (i.e. competitive) first order and first order autocatalytic decomposition on the critical parameter is reduceable to the single 
autocatlytic reaction format (i.e. $\left.f(n)=\left(\eta_{0}+n\right)(1-n)\right)$. Qualifications seem to be that the ratio of the first order rate constant divided by the first order autocatalytic rate constant must be small (i.e. Appendix B.10, references dealing with tetryl, [105, 109]). In cases where materials appear to melt with decomposition (Appendix C.2, ref. [149, 150]), the critical parameter is calculated from the maximum reaction rate which occurs when "melting" is just complete. 


\subsection{Hazard Tests}

\subsubsection{General Description of the Field}

To answer the second set of questions cited in the introduction in section 4.4 (i.e. are our definitions of thermal instability and thermal instability hazard consistent with the state of the art in hazard testing?), a synthesis of what seems to be the state of the art in the self-reaction hazard evaluation field (abbreviated $S R H E$ below) has been attempted. In the first part of Appendix B.11, our view of the broad picture of the field is summarized. This was constructed from work from such sources as government organizations (i.e. DOT, USA; RARDE, U.K., and RVO-TNO, Netherlands) concerned with safety regulations in transportation, the areas of handling and storage of industrial chemicals, fire research, and the handling and storage of industrial and military explosives. The spectrum of materials involved in these articles covers explosives, organic peroxides (near explosives), rocket propellants, and various unstable materials important in commerce such as ammonium nitrate fertilizers.

A coherent picture of the SRHE field could be constructed by sorting the tests according to the following criteria:

(a) Stimulus for initiation of decomposition: mechanical or thermal.

(b) Relationship of the particular stimulus to transportation accidents.

(c) Parameter measured: sensitivity to initiation of decomposition or damage potential.

(d) Purpose: reproduction of accident conditions or evaluation of physical properties of a material (fundamental parameter test). 
(e) Scaling: evaluation of the difference in behavior of materials in small scale tests and in bulk hazard conditions.

Details of the analysis are given in Appendicies B.II, C.I and C.2. Seven mechanical stimulus tests are summarized in Table B-4, ten thermal and ignition tests in Table $3-5$ and five types of calorimetric tests in Tables $\mathrm{C}-1$ and $\mathrm{C}-2$.

Two coments on the terms used above are pertinent before proceeding to more general matters. "Damage potential" tests measure the amount and rate of liberation of energy or gases associated with a thermal explosion, deflagration or detonation. As such they are very closely related to our definition of thermal instability hazard. "Fundamental parameter" tests involve the important assumption that the algorithm for predicting hazard characteristics is known.

Application of these eriteria made it possible to understand the otherwise bewildering array of. tests and to identify what each does. The criteria should be appropriate for any proposed tests. However, this study did not identify any single test that is sufficient for the determination of self reaction hazards posed by a wide variety of materials.

This is recognized by workers in the field. The self-reaction hazard rank of a material is constructed from a hazard profile based on a number of test results. Tests of both damage potential and sensitivity to decomposition by both thermal and mechanicai stimuli are involved. Tests on comercial chemicals are carried out on samples in various physical forms and with different amounts of chemical impurities in order to assess the effect on hazard rank due to these variables. Tests of the effect of the container are carried out since the degree of 
confinement can affect greatly the hazard rank. There are indications that the effect of the container will or can be of decisive importance in ranking the hazard of borderline unstable materials. Also, the concept that a reduction in the hazard rank can be obtained by appropriate packaging (which is known to apply to explosives), evidently applies to borderline hazardous materials also.

The current trend is to develop and incorporate more fundamental parameter tests results in constructing hazard profiles. This type of test can eliminate debates over the unambiguous meaning of "accident condition" tests as for example, the PVT test (No. 11 in Table B-5). This trend does not imply that there is a trend to eliminate "accident conditions" tests. For example, full scale tests, e.g., bonfire tests, are necessary (and sufficient) to resolve ambiguous hazard rankings associated with difference between small scale tests and bulk conditions. It was of interest to note that at least one experienced investigator felt the problem of attempting to improve the precision of test methods and results is compounded by the present lack of any quantitative data on thermal or mechanical stress in accident conditions.

\subsubsection{Thermal Sensitivity Tests}

Nineteen types of thermal sensitivity tests have been analyzed in order to determine what would constitute a sufficient single test or a series of tests comprising a test protocol for thermal instability. No single test now being used is sufficient. Three of these tests are noncalorimetric, but are in the fundamental parameter class: the thermal surge, test, the self acćelerating decomposition temperature test (SADT), and methods that determine the relationship between critical temperature and sample dimensions. They are summarized in Table $B-5$ and discussed in Appendix B.11. 
The thermal surge test is the pulse heating of a small, fully enclosed sample. Time to explosion and kinetic parameters are obtained. There is poor agreement with other techniques. Problems of interpretation limit its acceptability as a fundamental parameter test. The SADT, no. 15 in Table B-5, detects self heating in a material sized and packaged as it will be shipped. It has a major advantage in that no scale-up is required. Use of it has been restricted to organic peroxides. The results are a conservative measure of instability because the temperature of onset of self heating may be less than critical. The third set of non-calorimetric tests, critical temperature versus sample dimensions, is important because it provides the link between laboratory scale tests and bulk conditions. These tests show that themal explosion theory provides a suitable formalism for scale-up.

Of the remaining sixteen tests, three are applications of adiabatic calorimetry (Appendix C.1) and thirteen of heat conduction calorimetry (Appendix C.2). The principles of these calorimetric methods are described below.

\section{5 .3 Adiabatic Calorimetry}

Here the approach is to eliminate heat loss from the sample and its container. To do this the temperature of the environment is made to track that of the sample. The temperature of the sample is measured as a function of time. A typical system is shown in Figure 1. Approximate temperature equality is maintained either by heating a shield E electrically, or by forcing an externally heated gas through the shield and around the sample container 0 . The first method is used in the adiabatic storage test and the accelerating rate calorimeter (ARC). The second is used in the adiabatic self-heating test. Pressure effects are geasured only in 


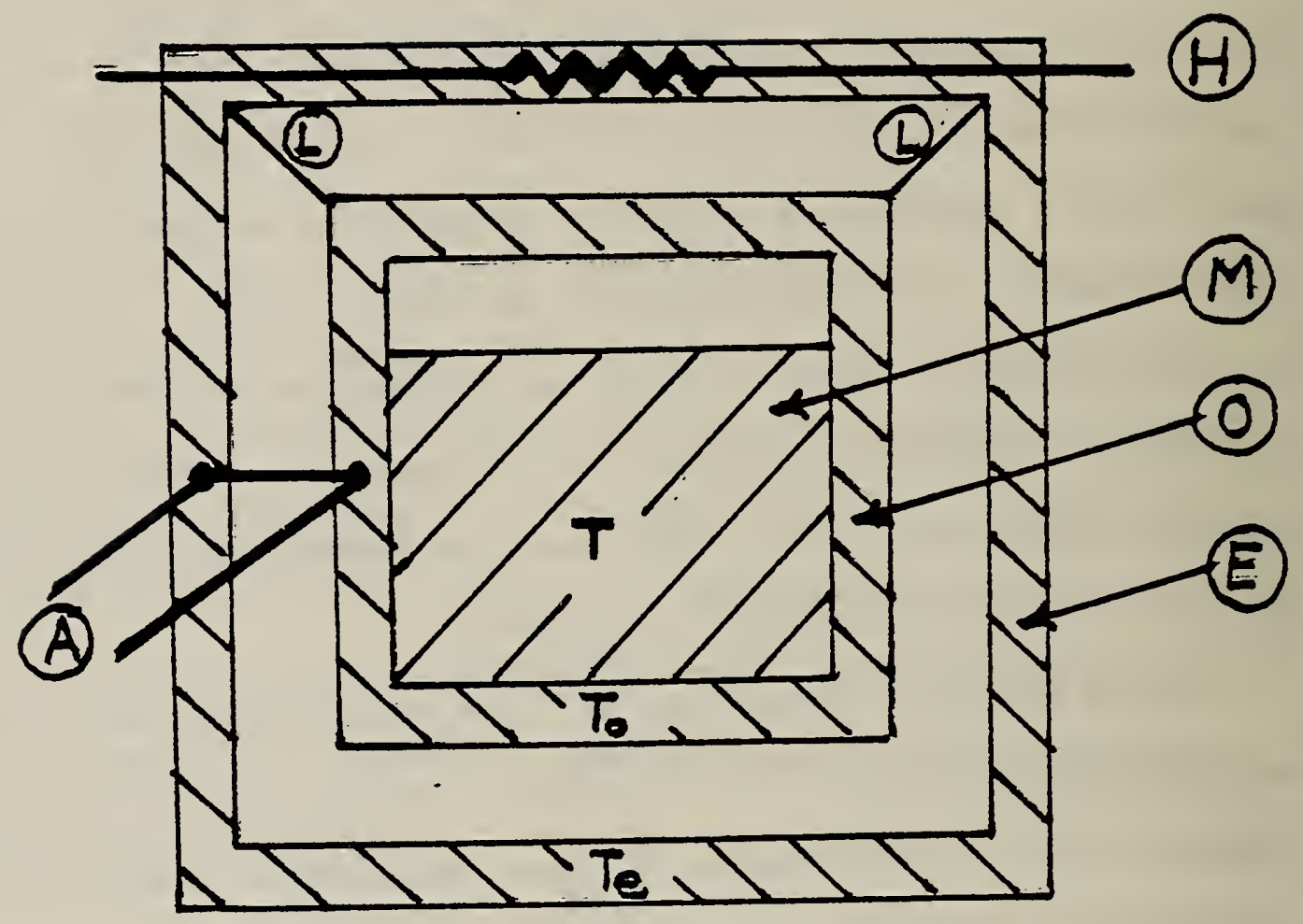

Figure 1. Sketch of Cross Section of an Idealized Adiabatic Calorimeter

A, difference thermocouple for controlling $T_{e}$ to be equal to $T_{o} ; E$, adiabatic shiald; $\mathrm{H}$, electrical heater for controlling adiabatic shield temperature; I, calorimeter supports; M, decomposing sample; 0 , sample container; $\mathrm{T}$, temperature of sample, $\mathrm{T}_{e}$, temperature of adiabatic shield; $T_{0}$, temperature of sample container; temperatures of sample, container, and adiabatic shield assumed to be uniform. 
the ARC, which has been used primarily for liquids. The other two tests use unconfined samples, but this does not appear to be a basic requirement. Rate constants and time to explosion can be extracted from adiabatic calorimetry tests. This requires careful interpretation of the results. If the kinetic mechanism is simple, rate constants can be extrapolated to other temperatures. If the mechanism is complex, or if appreciable decomposition occurs before explosion, interpretation of the results can be ambiguous, unless separate kinetic studiés are made.

In principle, these adiabatic calorimetry tests can model the thermal behavios of either a small mass of material in the center of a bulk shipment or that of a sample and its container. The nearly automatic elimination of temperature gradients in the sample is a major advantage that adiabatic calorimetry has over heat conduction methods.

\subsubsection{Heat Conduction Calorimetry}

Thirteen tests involving heat conduction calorimetry are sumarized in Appendix C.2. In heat conduction calorimeters, the heat liberated by the decomposition of the sample is measured directly by allowing it to flow to its environment only through a heat flow meter. The temperature of the environment is independent of that of the sample. It may be held constant (isothermal mode of operation) or be varied with time (programmed heating or scanning mode).

The basic design principle (see [157]) is shown in figure 2. The heat flow meter is a thermopile placed between the outer surface of the sample holder, $S$, and the inner wall of a block, $\Sigma$, whose temperature is controlled. The sample holder has.within it the sample, 2 , and its container, $i$. The thermopile output, $\lambda_{1}$, is linearly proportional to the race of heat liberated or absorbed by everything within the sample holder. 


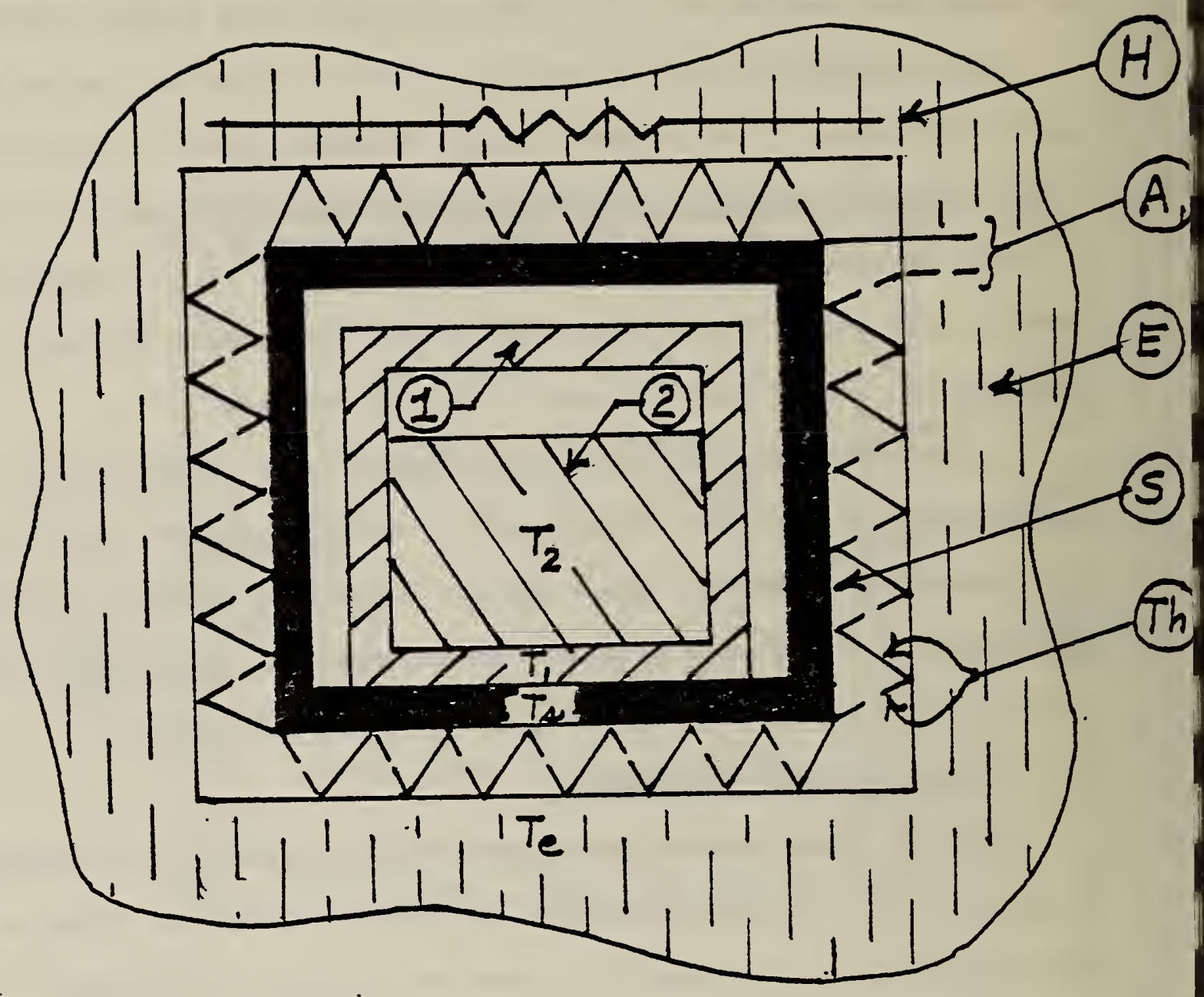

Figure 2. Sketch of Cross Section of an Idealized Heat Conduction Calorimeter

$A_{1}$, output of thermopile; E, block; $\mathrm{H}$, electrical heater for controlling block temperature; S, sample holder; Th, two elements of thermopile:

-, - -, different wires of thermopile; 1, sample container; 2 , decomposing sample; $\mathrm{T}_{b}$, controlled temperature of block; $\mathrm{T}_{\mathrm{s}}$, temperature of sample holder; $T_{1}$, temperature of sample container; $I_{2}$, temperature of sample; all temperatures assumed to be uniform. 
A thermal twin of the sample calorimeter (i.e., same thermopile, holder, container and inert reference material of the same heat capacity), mounted in the same block, often is used to cancel out variations in the sample thermopile output due to unwanted temperature fluctuations in the block. The differential output is linearly proportional to the differences in heat liberated or absorbed by the holders and their contents. Extremely sensitive single and twin heat conduction calorimeters are used in tests 1 and 2, respectively, of table C-1, Appendix C.2. To the extent that these types of calorimeters meet their design principle they have no calorimetric error associated with different geometrical locations of heat sources inside the sample holder.

Three simplified versions of the twin heat conduction calorimeter are applicable to hazard testing. These are "quantitative differential thermal analysis" (QDTA), "differential scanning calorimeter" (DSC), and "differential thermal analysis" (DTA). They are usually run in the programmed mode. They can be used for tests on samples that either absorb or evolve energy.

In QDTA the thermopile of each conduction calorimeter is replaced by a heat leak path and a single thermocouple, as shown in figure 3 . A temperature difference is measured. The heat leak must be calibrated. In this type of calorimeter, the energy calibration source must be both physically and thermally in the same location as the sample container if calorimetric error is to be avoided. Provided the latter condition is met, the output of the differential thermocouple is equivalent to the differential output of the thermopiles of figure 2 .

DSC is a compensated version of QDTA. A typical instrument is sketched in figure 4. Heat is supplied by electrical heaters in the sample and reference holders to keep the temperature of both of them at 


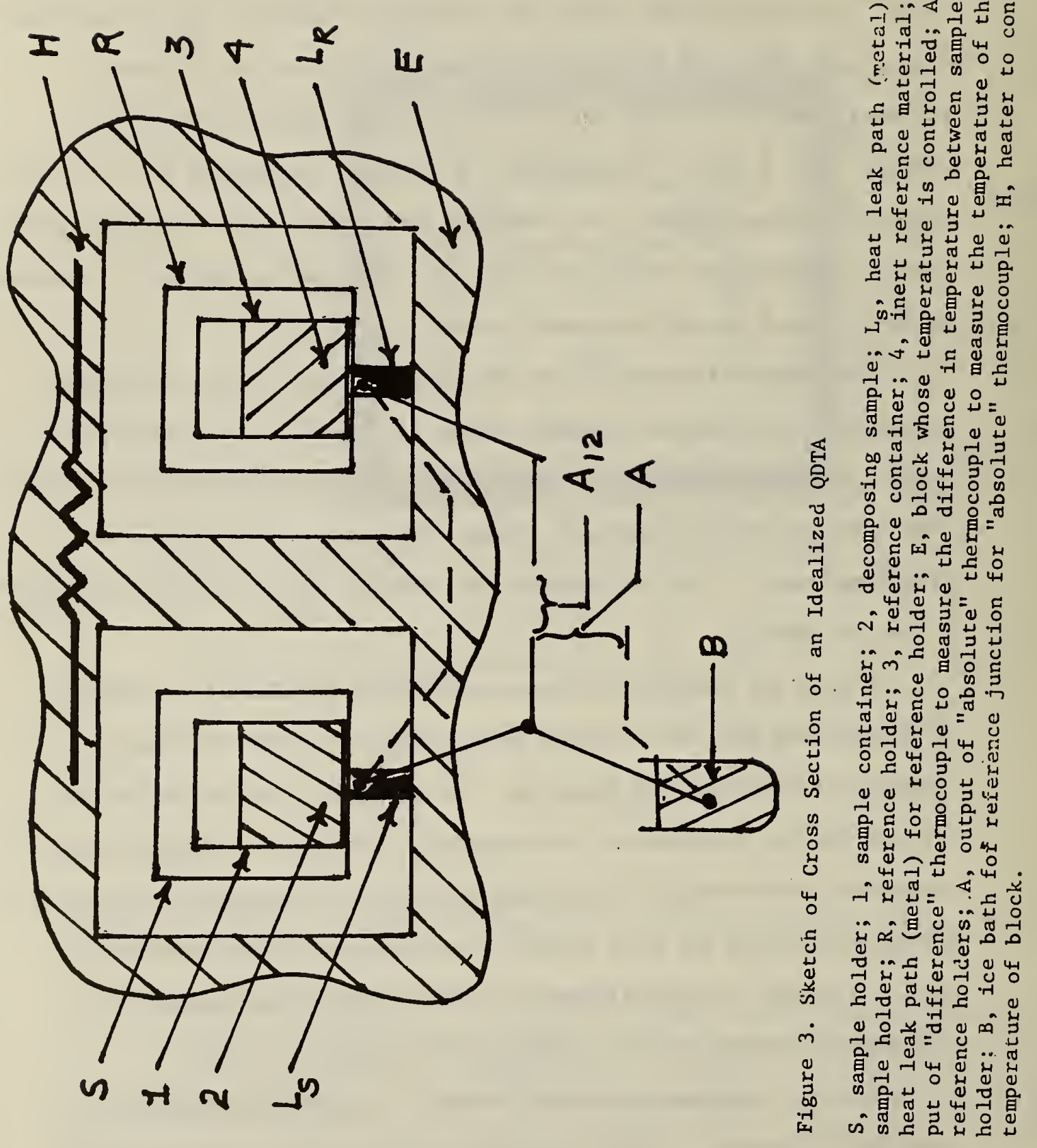




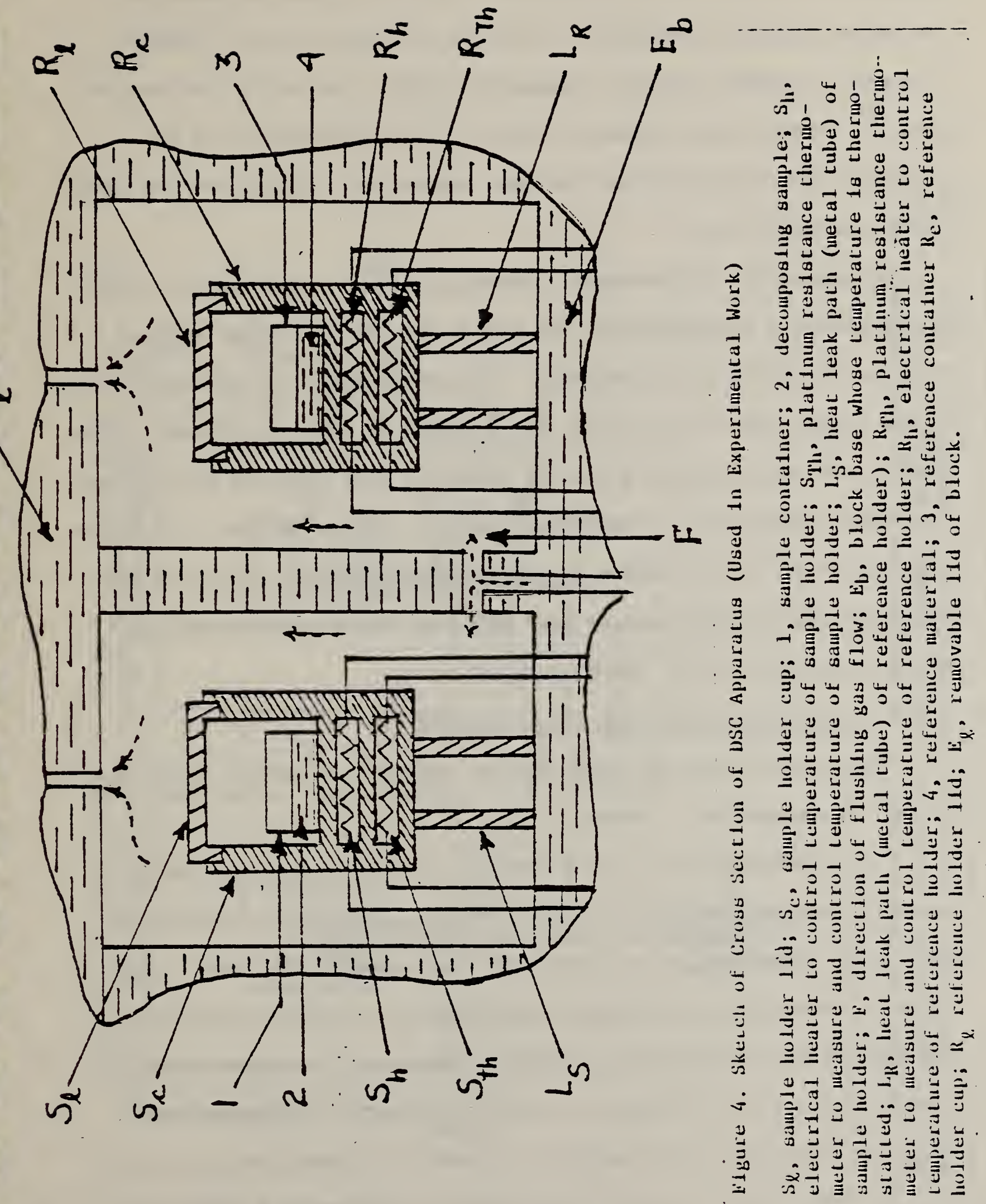


the same temperature and equal to a program temperature. The net difference in electrical power supplied to the two holders is measured. The major difference between DSC and QDTA is the electrical feedback circuitry, to which the term compensated refers, and which the name DSC does not convey. Both commercial QDTA and DSC instruments are considerably less sensitive than the heat conduction calorimeters mentioned above (see table $\mathrm{C}-1$ ).

In simple DTA (differential thermal analysis) measurements are made of the absolute temperature of the sample and the difference between it and that of the reference material. All are driven by programmed control of the temperature of the block, E. A sketch is shown in figure 5. For kinetic studies this method has the advantage over QDTA and DSC in that the sample temperature is measured directly. If the design is such that the temperature of the sample is kept uniform, this method can be made calorimetrically equivalent to heat evolution kinetic measurement involving either a QDTA or DSC measurement.

\subsubsection{Conclusions Concerning Hazard Testing}

These conclusions are based both on material in earlier sections and in the Appendices.

(1) Determination of the thermally instability hazard produced by thermally unstable materials is the aim of all the thermal sensitivity tests with the exception of flash point and ignitability tests. The critical temperature for thermal explosions is correctly identified as the maximum safe storage and handling temperature (i.e. where applicable - see section 4.3.6) in many but not all fundamental parameter tests (e.z. see tests 15,16 of table B-5, Appendix B.11 and tests 3, 4, 5b.1, $5 d .3$ of table $\mathrm{C}-2$ ). In the latter cases, the recommended maximum safe 


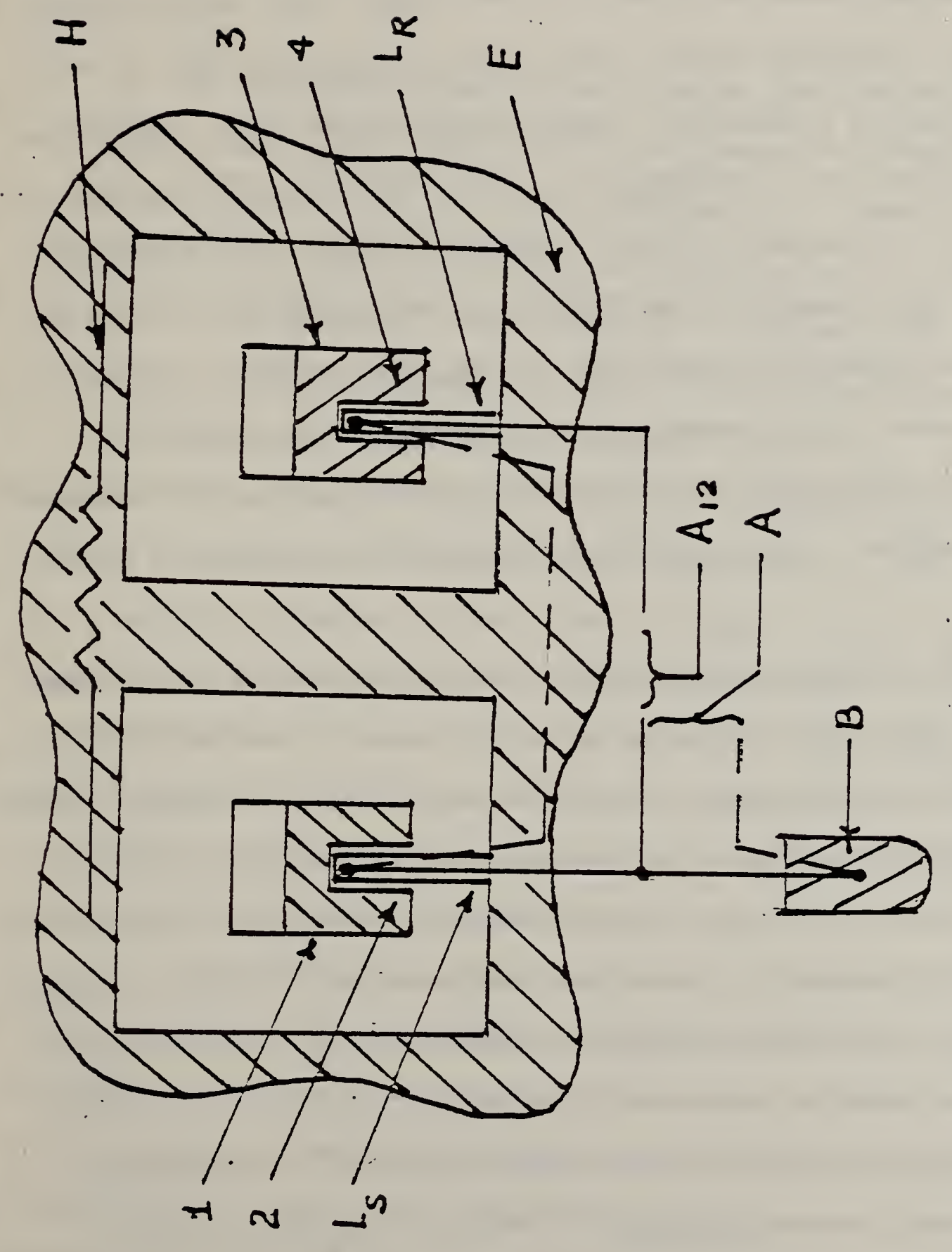

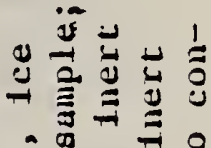

$\therefore=$

$\because 0$ ज

폰

-

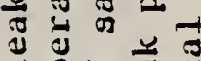

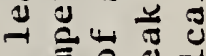

$\leadsto$ 苛

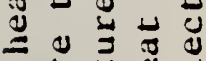

.

$\stackrel{3}{3}$

.. 巡 色

d)

节

$\rightarrow$ 일 일

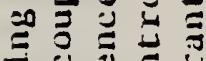

记

이넌

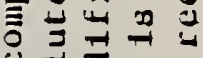

ของ

舫

N $=$ के

ఏ

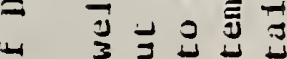

ว

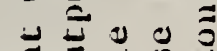

ว

ป

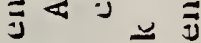

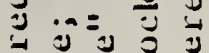

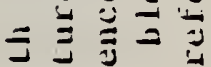

三 可 牙

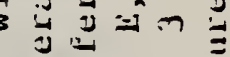

)

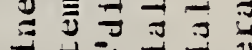

I $=-7$

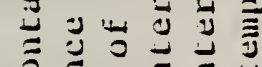

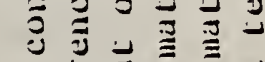

ง ข

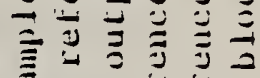

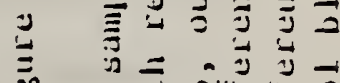

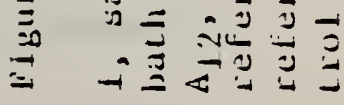


handling temperature generally turns out to be at or below the critical temperature. Thermal explosion theory is an explicit feature of the interpretation and application of thermal sensitivity tests that are advanced state-of-the-art for hazard evaluation (e.g. references and discussion of test 14,17 , table B-5; Appendix C.1; tests $1,2,5 \mathrm{~b} .2$, $5 c, 5 d .3,5 d .4$ of table $c-2$ ).

(2) Thermal sensitivity tests are only part of the tests required to construct a realistic hazard profile of a substance to rank its self reaction hazard in a regulatory transportation context (i.e. see discussion in first part of Appendix B.11, ref. [122, 124, 123]). This conclusion is consistent with work on general problem of safe handing, processing, and transport of materials (e.g. ref. [116, 128, 129]). It is clear that mechanical sensitivity and one or more types of damage potential tests (e.g. to measure rate and amount of gas evolution, determine the possibility of deflagration or detonation under confinement etc.) are required. This confirms and amplifies our conclusion in ref. $[8]$.

(3) The thermal sensitivity tests can be organized into "accident condition" tests and "fundamental parameter" tests. Those not involving local heating have the general purpose of determining the minimum uniform ambient temperature required to initiate a thermal explosion (unless gaseous decomposition product ruptures the container first -a possibility that can be calculated). The accident condition tests do this by direct measurements of the critical explosion temperature of the bulk material either in its shipping container (e.g., the SADT test, or in a thermal equivalent of the container (Warmestalagerung test). The fundamental parameter tests do this indirectly by measuring the heat evolution 
kinetic parameters ( $E$ and $A$ ) and in some cases the other characteristics of the material ( $\lambda$, thermal conductivity) or decomposition reaction ( $Q$, heat of decomposition per unit mass of reactant) needed to calculate the critical temperature. The fundamental parameter tests assume that the scaling relation (e.g. eq. 4 and some geometrical heat transfer variant of eq. 10) is known. This assumption is discussed in conclusion 3 , section $4 \cdot 4 \cdot 3$

(4) The fundamental parameter tests can be classified as calorimetric tests (Appendix C.I and C.2) or direct explosion temperature measurements (tests 14, 17, table 3-5, Appendix B.11). Some of the calorimetric tests explicitly involve the determination of $Q$ and $\lambda$ (i.e. Appendix C.I, the adiabatic self-heating test determines $Q, A, C$, and $\lambda$; Appendix C.2, test 5c. determines $Q$; test $5 d .3$ deternines $Q$ and $\lambda$ ).

(5) Three calorimetric thermal sensitivity tests (i.e. ref. [148], Appendix C.1, and tests 4 and $5 d .5$ of table $C-2$ ) determine the amount and rate of liberation of gases during the decomposition. Gas liberation is an important parameter for assessment of damage potential and a sensitivity test with this capability becomes equivalent in this regard to the fundamental parameter test 12 of table $B-5$. Since another parameter important in damage potential is $Q$, it seems clear that a thermal sensitivity test procedure should measure both $Q$ and gas liberation even though neither may have an important effect on $I_{\frac{1}{*}}$.

(6) The explosion temperature test developed by Bowes (see discussion, test 17, table B-5, Appendix B.11) has the important advantage that it addresses the problem of determining $E$ and $A$ under the equivalent of bulk conditions. It takés into account so called "model distortions" (Eroathing and foaming etc.), and addresses the problem of the scaling relation as a function of sample size. The difference in rate constants 
determined by this method and by isothermal calorimetric techniques on benzoyl peroxide, calorimetric values of $E$ were 10 to $20 \%$ greater, emphasize that all fundamental parameter tests must attempt to measure E, A, Q, etc., under conditions simulating bulk conditions. This necessarily implies conclusions (7) through (9).

(7) Small scale explosion temperature tests to check calculated values of $T_{*}$ or evaluate $E$ and $A$ from $T_{*}$ should be run under conditions when the effective Biot number is small and is known (i.e. see ref. [104] and Appendix B.10). This conclusion constitutes a major objection to the thermal surge test (test 14, table B-5) apart from the fact it determines the onset of container rupture rather than thermal explosion. (The other objection to the thermal surge test, that it gives values of $\Xi$ in error by $50 \%$, may be due in part to incorrect data analysis, see Appendix B.11). The explosion temperature test devised by Rodgers [149] (see Appendix C.2) apparently does not meet this low Biot number requirement.

(8) Small scale measurements of $E$ and $A$ in calorimetric measurements must, in general, be run under conditions of minimal Biot number. This is automatically fulfilled in adiabatic calorimetry but usually is not an explicit part of heat conduction calorimetric measurements.

(9) The predictions of the critical temperature made from parameters measured in calorimetric tests should be checked directly by, explosion cemperature measurements as specified in conclusion ( 7 ). If the Bowes method (discussion, test 17 , table B-5, Appendix B.11) is used, $\mathrm{E}$ and $\mathrm{A}$ should be checked with calorimetric measurements. Since explosion temperature measurement's are time consuming, it seems clear that it is expedient to run the calorimetric measurements first. The explosion temperature tests must evaluate the effect of sample size. This should je mandatory if the material decomposes in the solid state (in which 
case particle size is important) or if there is a possibility that reaction mechanisms are affected by sample dimensions (erg. diffusion).

(10) It is clear from studies on explosives (see Appendix B.10 references dealing with tetryl, [105, 109]) that possible catalysis and/or alteration of heat evolution mechanisms by gaseous reaction products when kept in contact with reactants dictate that colorimetric tests must have provision so that they can be run under closed, small free volume, conditions: This is explicitly provided in one adiabatic colorimetric test (ref. [148], see Appendix C.1). This should be a requirement if maximum hazard or unvented container conditions are to be determined.

E? 


\section{Experimental Work}

\subsection{Introduction}

An experimental study of the decomposition of thermally unstable materials was carried out to identify what the various detailed components and specifications of an experimental test procedure should be in order to rank thermal instability as defined in section 4.4. The specific test method that became the focal point for the work is, a test method proposed by ASTM Committee E-27 on Hazard Potential of Chemicals. It is an application of differential thermal analysis. The method has the advantage that it has been proposed by workers experienced in the field of self-reaction hazards and direct interlaboratory comparisons were possible. The materials studied included some of those used in an ASIM round robin test of the method ${ }^{+}$.

In effect, three studies were carried out in parallel. The first study was an analysis of the operation and capabilities of the commercial DSC apparatus (Perkin-Elmer, Model DSC-2) used in the second study, the actual experimental decomposition work using the proposed ASTM method. The third study was an evaluation of the limitations and possible extensions of the test method in context of its use as part of a test procedure for thermal instability.

The test method and the assumptions underlying it are discussed in section 5.2. The operation and calibration of the instrument are summarized in 5.3. Experimental results are given in section 5.4 and discussed in 5.5. Conclusions and recommendations for future experimental work are collected in section 5.6 .

\footnotetext{
tive are indebted to Dr. Allen Duswalt of Hercules, Inc. for supplying the test materials.
} 
The discussion and analysis of sections $5.2,5.3$, and 5.5 and the related appendices C. $3, C .4$, and C.5 apply to extended arguments that were developed from a study of number of literature sources. These are the ASTM test method documentation [158], the critiques or reviews of ref. $[152,159-165]$, and three texts [166-168]. 


\subsection{Summary and Assessment of the proposed ASTM Test Method ${ }^{+}$}

The purpose of the proposed test method is to determine the kinetic parameters of materials that decompose with liberation of heat in order to evaluate their potentials to undergo thermal explosions. (Reaction of the material with the surrounding atmosphere, e.g., autooxidation, could be considered to be within the scope of the method, although this is not specifically stated.)

The method is an application of differential thermal analysis based on a procedure introduced by Murray and White (169) and extended by Kissinger (170). Later analyses of it by Ozawa (158) are cited in the test procedure. The basic method was developed for the study of endothermic decompositions and phase transitions.

In this method samples weighing a few milligrams are heated at various constant rates, $w$, in a series of runs. The temperature at which the maximum rate of decomposition occurs, $T_{d}$, is determined for each run. The sets of data points ( $w, T_{d}$ ) are processed to give the parameters of the Arrhenius equation, $k=A \exp (-E / R T)$.

The method is stated to apply to materials that decompose by a single exothermic reaction for which the simple general rate law

$$
E(n)=(1-\eta)^{n}
$$

is applicable. $n$ is the order of the reaction and is greater than or equal to zero. The method is specifically stated not to be applicable to reactions that are autocatalytic or to decompositions in which the material undergoes phase transitions in the same temperature range as that of the decomposition. That is, at least any process that shows a complex exotherm is to be excluded.

\footnotetext{
This description of the method is based on the third draft of the proposed procedure.
} 
The data analysis is based on the kinetic model

$$
d \eta / d t=f(\eta) k(T)=f(\eta) A \exp (-E / R T) .
$$

The maximum value for the observed exotherm and that for the rate of reaction, $\mathrm{d} n / \mathrm{dt}$, are assumed to coincide. Eq. 11 is differentiated with respect to time to identify this maximum and rearranged to yield

$$
g\left(n_{d}\right) \equiv-(d f(n) / d \eta)_{d}=\left(k^{-2}(d k / d T)(d T / d t)\right)_{d}=\left(w E / A R T_{d}^{2}\right) \exp \left(E / R T_{d}\right)
$$

The term $\mathrm{dT} / \mathrm{d} t$ is set equal to the programed scanning rate, $w$.

Assuming that $g\left(\eta_{d}\right), E$ and $A$ are constants, the basic equation of the method is obtained by differentiating eq. 12

$$
d 2 \mathrm{n} w / d\left(L / T_{d}\right)=-(E / R)\left(1 /\left(1+2\left(R T_{d} / E\right)\right)\right)=-E / R D
$$

The activation energy, E, is extracted from the slope of a plot of $\log _{10} \mathrm{w}$ versus $1 / \mathrm{T}_{\mathrm{d}}$ using the formula

$$
E=-2.303(R / D)\left(\log _{10} \mathrm{~W} / \mathrm{d}\left(1 / \mathrm{T}_{\mathrm{d}}\right)\right.
$$

where $D$ is defined in eq. 13. An iterative procedure is used starting with an initial value $D=1.051$ and a value of $T_{d}$ near the middle of the experimental temperature range.

The preexponential factor, $A$, is obtained by applying eq. 15

$$
A=\left(w E / R T_{d}^{2}\right) \exp \left(E / R T_{d}\right)
$$

to each data point pair and the mean value is determined. This equation is a rearrangement of eq. 12 , with the assumption that $g\left(\eta_{d}\right)=1$.

The kinetic parameters are checked experinentally in an "ageing" run. The temperature at which $50 \%$ decomposition would occur in about one hour is calculated, assuming a first order reaction. A sample is aged for that period and then scanned. If the total heat evolved is one half the amount that would be evolved by the sample without aging, the kinetic analysis is considered to be verified. This ageing experiment extends the range of measurements to siightly lower temperatures. 
The experimental apparatus is required to be a QDTA or DSC, as the terms are defined in section 4.5.2. The procedure states that commercial instruments manufactured by Perkin Elmer (DSC) and DuPont (DuPont 900 or 990 including the DSC module) are suitable. The sample is kept small to minimize temperature gradients within the sample. A sample weight resulting in a heat generation rate of $8 \mathrm{~mW}$ or less is stated to be satisfactory, apparently in terms of the above commercial apparati. The sample also is to be placed in good thermal contact with and in a container with which it will not react. The atmosphere around the sample should represent the conditions of usage and either a hermetically sealed or high pressure container is required for volatile samples.

The method described above has been applied in our experiments, due allowance being made for instrumental corrections.

Several aspects of the test method and the model merit discussion here. Others will be covered later.

1. The Arrhenius rate expression is assumed. This is used widely in kinetics, with a high degree of success. It is appropriate for the analysis of data over a limited temperature range, but may not be adequate for long extrapolations. Deviations from this rate expression, substantial non-linearity in the $\ln _{\mathrm{W}} \mathrm{vs} .1 / \mathrm{T}_{\mathrm{d}}$ would be evidence for failure. But experience in kinetics indicates that very precise data are needed to show such deviations for simple reactions and that the more common cause of non-linearity is a change in mechanism with temperature, i.e., a change in the form of $f(\eta)$.

2. The calculation of the preexponential factor, A, using eq. 15 requires $g\left(\eta_{d}\right)$ in eq. 12 to be constant and equal to one over the experimental temperature range. This assumption is examined in Appendix C.5.I. 
There it is shown to depend upon $\mathrm{dT} / \mathrm{dt}$ for the sample being equal to the programmed scanning rate during the entire exotherm. Assuming the scanning rate to be constant, $g\left(\eta_{d}\right)$ is shown to be unity or close to it for reactions of simple order and to vary slightly with reaction order. (It may deviate appreciably from unity for autocatalytic reactions.) This analysis yields an extention of equations 13 and 14 which are used to calculate E. Again, the corrections are small, when the simple rate law, eq. 8, applies.

3. The heating rate for the sample, $d T / d t$, cannot equal the scanning rate, w, exactly during the exotherm. The errors introduced by this self-heating are discussed in Appendix C.5-2 and an upper bound on the error in $\mathrm{E}$ is developed. Briefly, for reactions of interest here, the error in $E$ would be less than $2 \%$ for a temperature rise due to selfheating of less than $0.2 \mathrm{~K}$.

4. No specification in the test method addresses the required constancy of $d T / d t$ during a scanning run (see above) except possibly the peak power limitation of $8 \mathrm{~mW}$. In any event, some requirement needs to be identified. It is clear from the critiques by Garn (see [159], p. 82) and Sharp ([160] section III, A.1) that the assumption of a uniform sample temperature is or can be a major source of error. Thus, also identifying precisely how the peak power specification limits this error seemed to be of paramount importance.

5. Understanding the physical basis for the temperature lags in the test method is important, although these lags apparently are small. They are relevant to calibration of the temperature scale of the instrument and are related to the use of metallic melting point standards in determining the transfer function of the instrument (i.e. correction for time lags in the ordinate or energy signal). 
6. Ozawa [158], Garn ([159], p. 85), Sharp ([160], II, B.) and Wendlant ([166], p. 189) indicate that errors of varying magnitudes can be made in assuming that the peak of a DTA curve coincides with the maximum in $\mathrm{dn} / \mathrm{dt}$. (Much of the comment is based on the work of Reed, et al ([172]). Although the error may be small for QDTA or DSC [158], the assumption of coincidence could be violated.

7. Methods are needed to determine a) whether the reaction is complex (and how to interpret the data in that case), b) how far outside the experimental temperature range the data may be extrapolated, and c) the form of $f(\eta)$ for both simple and complex cases. Ideally these methods should be independent of assumptions about the constancy of $\mathrm{dT} / \mathrm{dt}$ (an essential feature in refs. [161, 162]) and about the form of $k(T)$. These assumptions can be avoided when the reaction is not complex if $\eta$ and $d \eta / d t$ are measured. This is discussed further in section 5.6. 


\subsection{Instrument Evaluation and Calibration}

Two types of instruments, QDTA and DSC, are permitted in the procedure studied here. It is important to determine the conditions under which they yield the same result, how the data should be reduced in each case, and, in general, how they operate. Neither measures the temperature of the sample directly. Heat losses, electronic circuitry and definition of the temperature calibration all are involved in interpretation of the instrumental output. Errors may be introduced by approximations in the models.

The theory of operation of QDTA and DSC instruments is given in Appendix C.3. That section presents (1) energy balance and signal response equations, (2) methods for determining $\eta, \mathrm{d} n / \mathrm{d} t$ and the temperature of the sample (when the reaction is simple), (3) methods for calibrating the energy and temperature scales of a DSC, and (4) the effect of time constants on the signal. The calibration of the instrument used here is presented in Appendix C. 4 and then the determination of the transfer function of the instrument is discussed. Qualitative results of these analyses are given below.

The simplified QDTA system that was analyzed has the sample and reference containers and holders in the same compartment. It was found that this lack of isolation introduces negligible error provided that the corresponding holder and container are in good thermal contact.

It was found that the form of the measurement equations for the QDTA and DSC were formally identical in terms of the ordinate or energy signal of either instrument after it has been corrected for instrumental 1ag. This was expected and is consistent with ref. [175]. 
Two methods for determining $\eta, d \eta / d t, T$, and the heat ${ }^{+}$of decomposition were worked out in terms of the energy signal, the heat capacity of the sample and sample container, and the heat transfer coefficient between sample and sample holder. These are summarized in Table 22, Appendix C.3. The least restrictive assumptions for acceptable performance compatible with parameters that can be characterized experimentally are that the temperature of the decomposing material and its container be the same and uniform. Provided volatile products are brought into good thermal contact with the container before they escape, the container can be either open or closed. (If results differ under these open and closed conditions, the mechanism of decomposition is different when reaction products escape.) Keeping the thermal contact between a unit area of the sample and its container large keeps the difference between the temperatures of the sample and container small. Conversely, keeping the thermal contact between the sample container and the sample holder small helps to reduce temperature gradients in the sample. At least one necessary qualification is that the decomposition is or mimics a homogenous reaction (so eq. 11 applies -see Appendix, B.6).

The calibration of the energy scale of our DSC (determination of the calibration constant, $K$ ) was made with the known heats of fusion of indium and tin and the known heat capacity of $\mathrm{Al}_{2} \mathrm{O}_{3}$ (NBS standard Reference Material 720). $\mathrm{K}$ was independent of temperature within the uncertainty of the measurements. This is consistent with the design and specifications of the instrument.

\footnotetext{
†epending upon circumstances, either the enthalpy or energy of decomposition at a specified reference temperature.
} 
The temperature scale and time lags of the instrument were determined using the indium and tin fusion points. The static temperature calibration was determined from the intercept of a plot of the so-called onset temperature, $I_{\text {on }}(w)$, versus scan rate, $w$. The slope of the plot was interpreted as suggested by Flynn [191]. Equations are worked out in Appendix C.3.4 to support his interpretation. The assumptions were 1) the sample and sample container temperatures are uniform and constant during melting, 2) the sample and sample container temperatures are equal, 3) the ordinate transfer function of the instrument is a first order differential equation involving the time constant, $\tau_{d}$, of the differential power circuit, and 4) the sample holder temperature lags behind the program temperature by the amount $\tau_{a} w$ where $\tau_{a}$ is the time constant of the average power circuit and $w$ is the nominal scanning rate.

An upper bound of the time constant of the differential power circuit was determined from the exotherm of partially supercooled indium in a cooling run.

Equations based on the assumptions of the $I_{\text {on }}(w)$ analysis are presented for evaluating the varying temperature of the sample during a scanning decomposition run. They show that the temperature calibration procedure prescribed for the ASTM kinetic method cortectly accounts for the temperature corrections apart from self heating provided the following conditions are met: 1) $\tau_{d}$ wust be small in comparison to $\left(\tau_{a}+\tau_{s}\right) w$ where $\tau_{s}$ is the time constant of the sample container and 2) the heat capacities of the samples in the temperature calibration and decomposition runs are small in comparison to those of the sample containers. (For other restrictions see Appendix C.3.4.) 
The heat transfer coefficient between the sample container and holder, 'h' is ' is needed to determine the temperature offset of the sample and its container due to self heating. This can be calculated from the leading edge of pure metal endotherms as both Flynn's [191] and our analyses of the $T_{\text {on }}(w)$ versus $w$ plot show. However, this approach is not correct if the second assumption of the $T_{\text {on }}(w)$ analysis does not hold during melting. A test for the validity of this second assumption was developed and applied to the experimental endotherms for indium and tin. The test showed the temperature of the sample and its container are equal for the purposes of calculating h'

A more exact analysis of the DSC energy balance and electrical power feedback equations was carried out. Results to date are: 1) assumptions 3 and 4 of the $T_{\text {on }}(w)$ versus $w$ analysis are valid to a good first approximation, 2) the condition for the time derivative of the sample holder to always equal $w$. is that given by $0^{\prime N e i l l ~[176] ~ p r o v i d e d ~}$ certain measurable non linear terms in the equation relating the program and holder temperatures are small, 3) the ordinate signal correctly accounts for the rate of heat liberated or absorbed by the sample provided certain measurable non linear terms in the equation for the ordinate signal are small. The first result provides support for the validity for the temperature corrections in section 5.4 and the error analysis in section 5.5. The condition of the second result holds for both the melting point and decomposition studies. Thus, essentially all the measurement equations we have formulated which involve this assumption should.be correct in this regard. The third result confirms an argument given by $O^{\prime}$ Neill [178] and extends it by suggesting what to measure if there is doubt. Applied to our studies, the nonlinear therms in the third result are negligible. 


\subsection{Experimental Work}

Preliminary experiments were run on a sample of polyacrylonitrile (supplied by A. Duswalt, Hercules, Inc.) and three analytical reagent grade oxidizers: ammonium nitrate $\left(\mathrm{NH}_{4} \mathrm{NO}_{3}(\mathrm{c})\right)$, potassium persuifate $\left(\mathrm{K}_{2} \mathrm{~S}_{2} \mathrm{O}_{8}(\mathrm{c})\right)$, and ammonium dichromate $\left(\left(\mathrm{NH}_{4}\right)_{2} \mathrm{Cr}_{2} \mathrm{O}_{7}(\mathrm{c})\right)$. The purpose of the experiments was to become familiar with the problems in studying decomposition reactions. Samples were run in a variety of containers including heremetically sealed disposable aluminum containers (Perkin Elmer Part 219-0061) with a burst pressure of 2-2.5 atm. The instrument was run with the standard block lid with a flow of dry $\mathrm{N}_{2}$ gas of roughly $20 \mathrm{cc} / \mathrm{min}$.

A smaller exotherm was observed for $\mathrm{NH}_{4} \mathrm{NO}_{3}$ (c) in a sealed container than that expected. This was apparently caused by lack of confinement and/or volatilization of the material. In the case of polyacrylonitrile, vaporization of the decomposition products caused a large change in heat lost by the sample holder. Products condensed on the ceiling of the sample holder chamber. This evidently resulted in a change in the emissivity of the surface and thus increased the rate of radiant heat loss by the sample holder. To illustrate, the difference in the steady output signal of the instrument between the initial and final isothermal temperatures of an initial blank (i.e. empty holders) scanning run was preset at $0.2 \mathrm{~mW}$. The observed difference for a scanning decomposition run was $4.3 \mathrm{~mW}$, see fig. 6 . The heat loss was progressively altered in an unknown way to a final value of $4.1 \mathrm{~mW}$. Since the rate of heat generation was only on the order of $5.8 \mathrm{~mW}$, it was evident that this extraneous emissivity effect had to be reduced. The results on $\mathrm{NH}_{4} \mathrm{NO}_{3}(\mathrm{c})$ indicated that a sample container with a higher burst pressure than 2 atm and a temperature range with an upper limit of $500 \mathrm{C}$ was needed. 


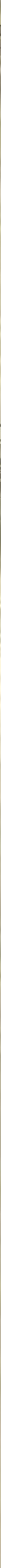


The high pressure cells that have been used in DSC or QDTA studies are summarized in Table 1 . One reusable and two disposable containers are available commercially; only the disposable containers fit into the sample holder, with holder lids in place, of our instrument $(0.790 \mathrm{~cm}$ I.D. and $0.32 \mathrm{~cm}$ inner depth). A reusable container was designed that would fit into the existing holders in order that holder lids could be used $^{+}$. A prototype cell fabricated from 750-X inconel appears to be satisfactory. It was pressure tested to $80 \mathrm{~atm}$ and changed weight less than $5 \mu \mathrm{g}$ after a first cycling between ambient and $500 \mathrm{C}$ in a dry nitrogen atmosphere. The gold disc seal must be replaced every two or three runs. A preliminary run of the decomposition of polyacrylonitrile confirmed previous observations that the difference in isothermal signals was due to a change in heat loss by the sample holder. Because it was clear that further time was needed to perfect, characterize, and replicate the high pressure cell for routine use, experiments using the cell and studies on polyacrylonitrile was stopped. We mention this development work because it was clear at the outset of the experimental work that a closed reusable cell was also needed to indicate maximum hazard conditions (see conclusion 2 of section 5.6). Comments on the needed further development and testing of the cell is given in Appendix C.6.

\footnotetext{
The instrument can be run without holder lids provided the same reusable containers are used in calibration of the energy and temperature scale of the instrument. The containers must have the same emissivity and the same thermal contact with the sample holder in both decomposition runs. The possibility of systematic error due to temperature gradients is, however, much greater than when holder lids are used.
} 


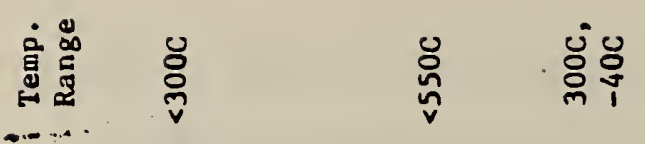

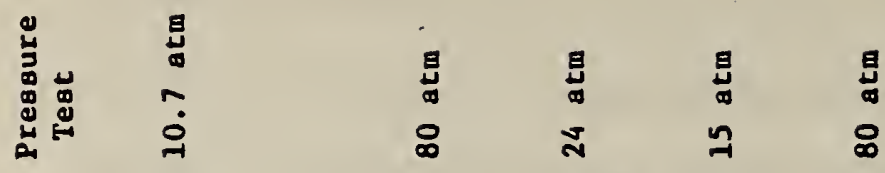

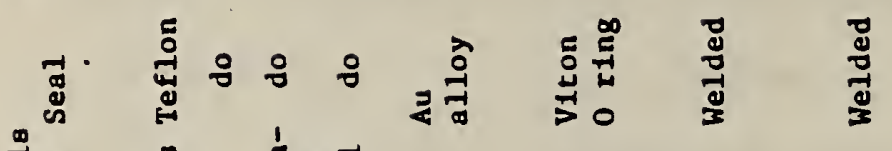

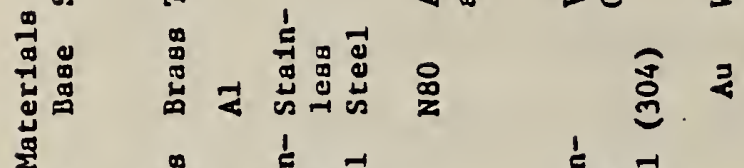

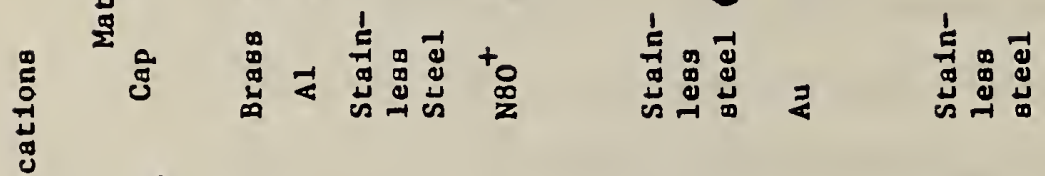

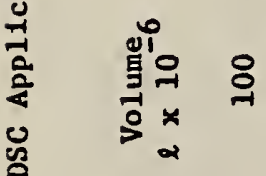

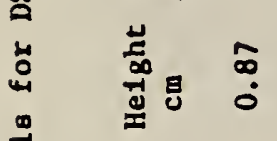

$\circ \approx$

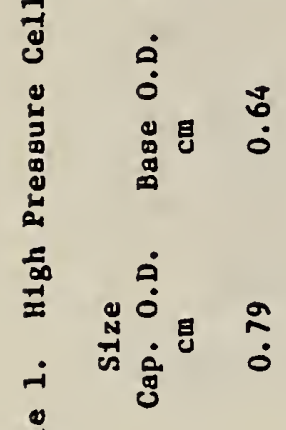

กับ

a

要

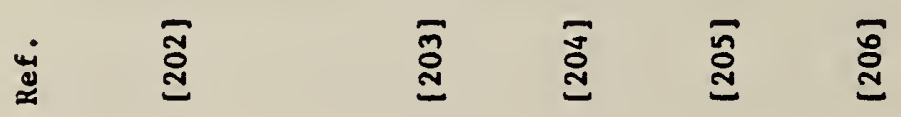

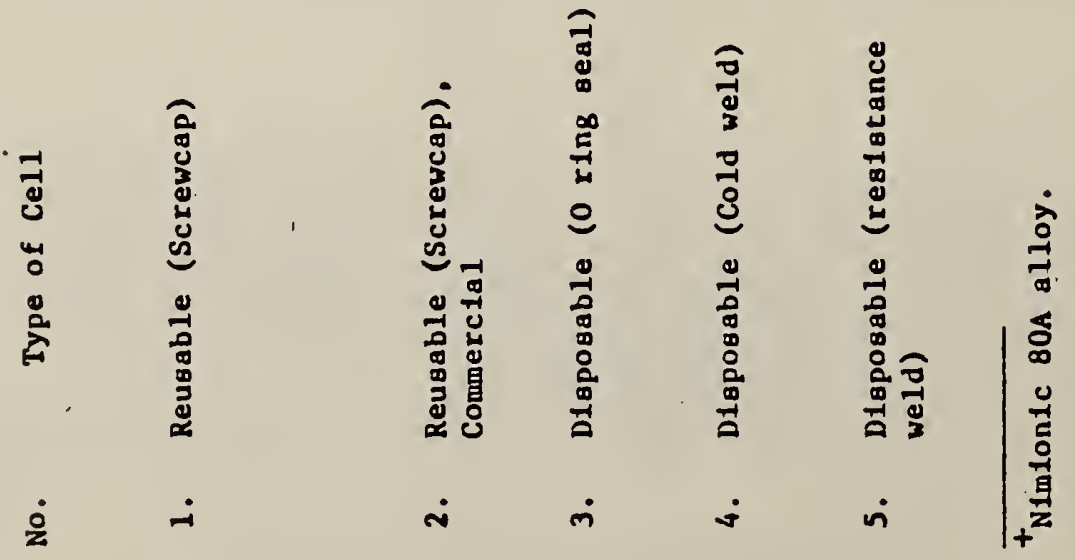


Nitrocellulose film, m-azidobenzoic acid, and azidotriphenylmethane (supplied by A. Duswalt of Hercules, Inc.) were sealed in their containers in air. Two types of containers were used. Crimped but open aluminum sample containers (Perkin Elmer part 219-0041) were used for nitrocellulose as shown in fig. 7b. No emissivity effect, as described for polyacrylonitrile, was observed and scanning runs were carried out with the standard block lid and $20 \mathrm{cc} / \mathrm{min}$ of dry nitrogen flushing gas. In the case of both m-azidobenzoic acid and azidotriphenyl methane samples were placed in hermetically sealed cells (Perkin Elmer part 219-0062). Good. thermal contact between sample and cell was promoted by sandwiching the sample between the bottom of a cell and an aluminum disc forced into the cell as shown in figure $7 \mathrm{a}$. (This procedure was recommended by A. Duswalt.) In the case of both m-azidobenzoic acid and azidotriphenylmethane, small emissivity effects were observed. However, they were eliminated by using a modified block lid supplied by the instrument manufacturer. The purge gas flow is substantially greater than in the standard arragement and deposition of products on the lids causes less change in radiant heat loss by the sample holder.

Sketches of exotherms for m-azidobenzoic acid, azidotriphenylmethane, and nitrocellulose are given in figures 8 to 10 . The results for the ASTN test are summarized in tables 2 to 4 . The various symbols and their meaning are given in table 5. The nominal exotherm peak temperature, $T_{d}$ (nominal), in column 4 is the instrument program temperature corresponding to the exotherm peak of the observed output signal, 0 , in the decomposition run minus the output signal, $0_{0}$, in a subsequent blank run when only reaction products are present in the sample container. The uncertainty for column 4 is estimated to be 0.1 to $0.2 \mathrm{~K}$ at minimum and on occasion may have approached $0.5 \mathrm{~K}$ to $1 \mathrm{~K}$. 

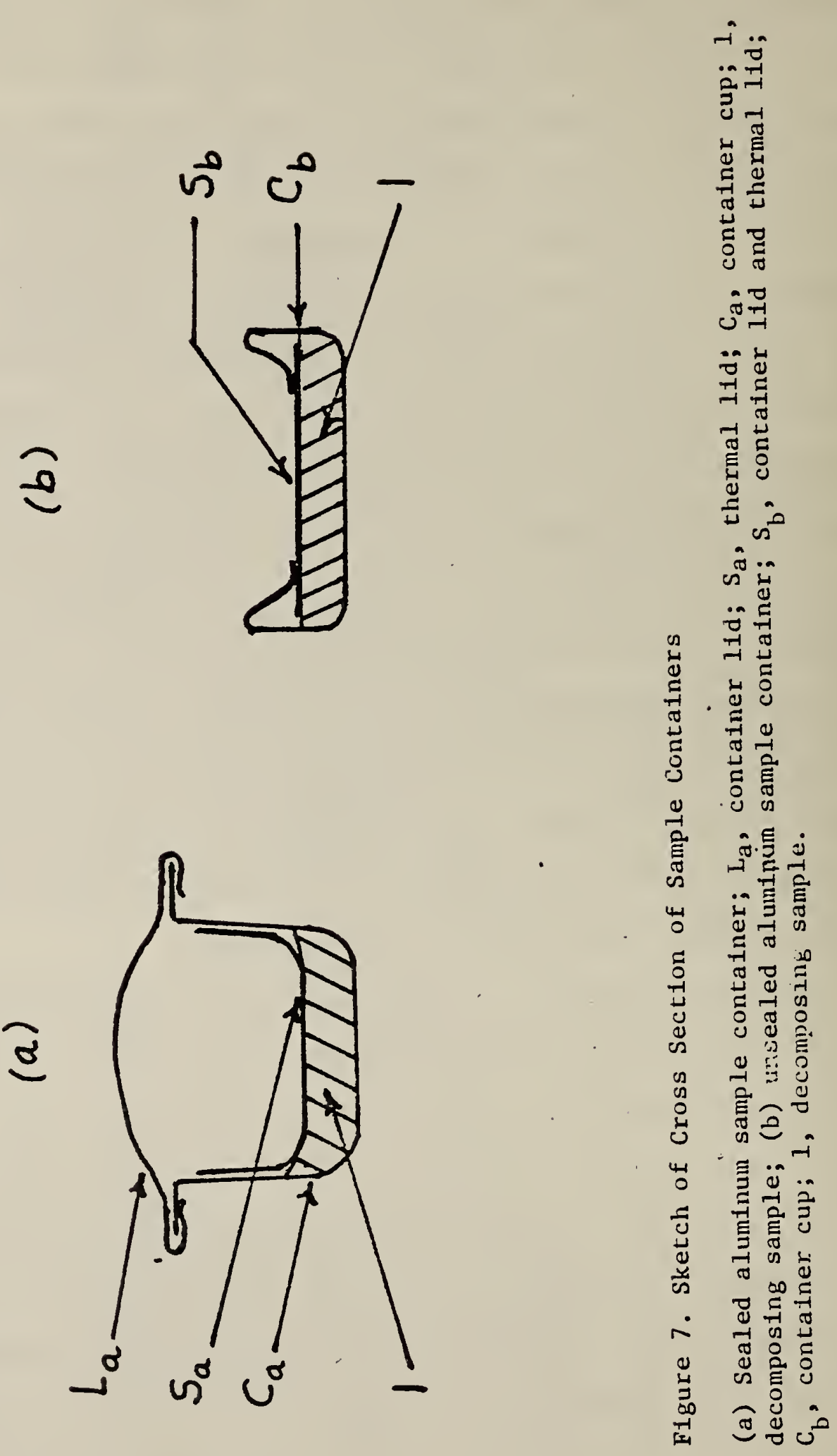


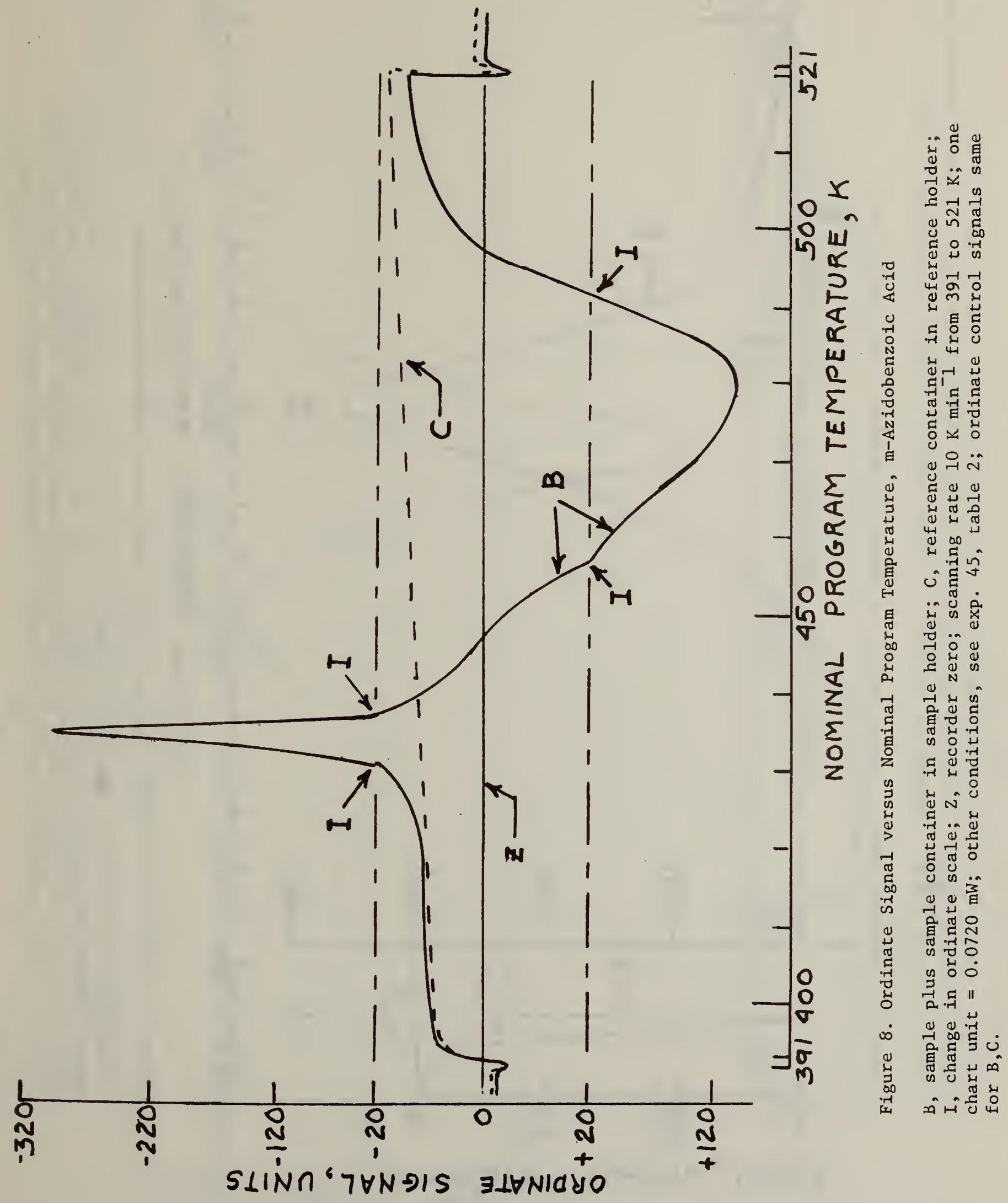




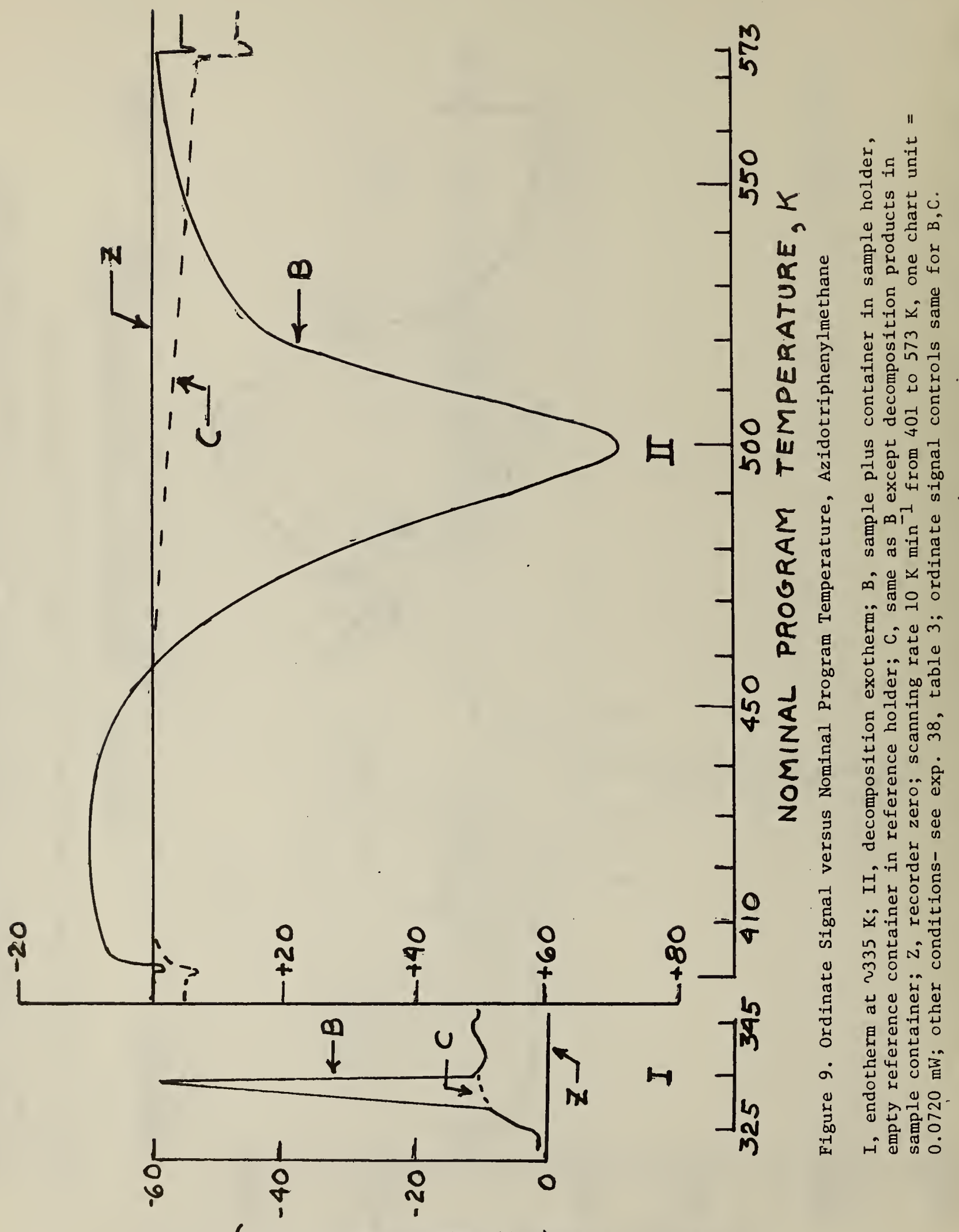

SLINก $6 \forall N D I S \quad \exists \perp \forall N I O \forall O$ 


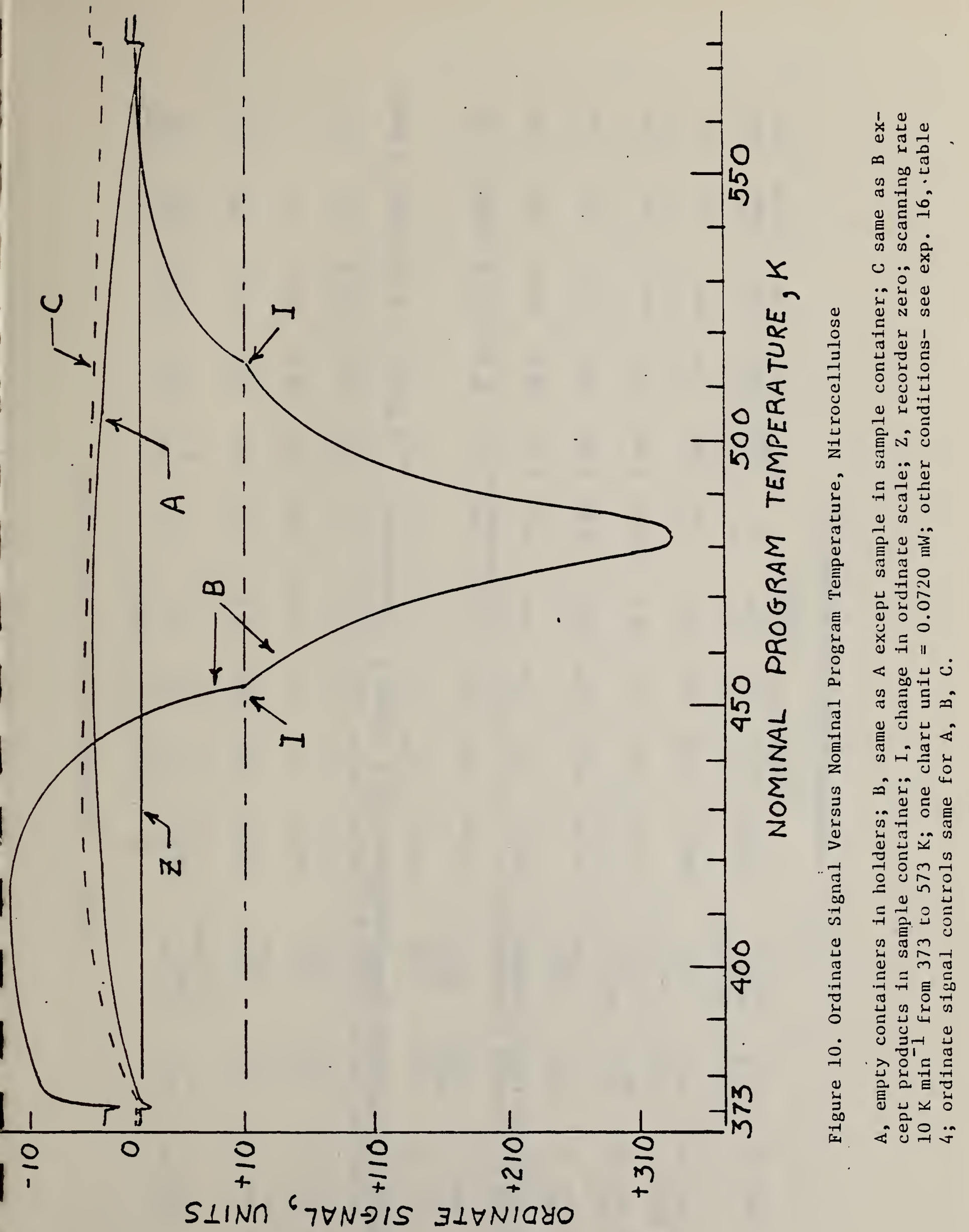




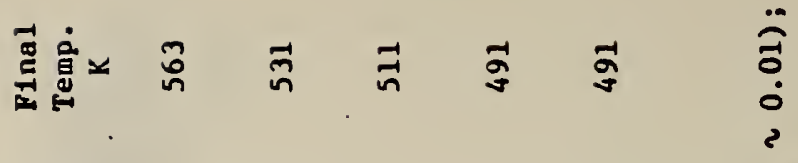

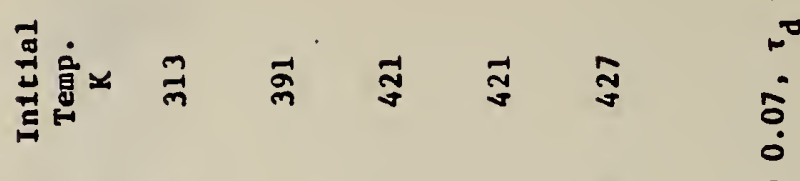

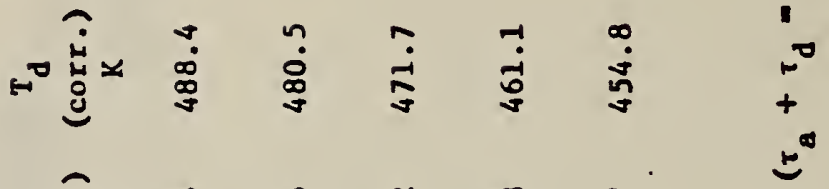

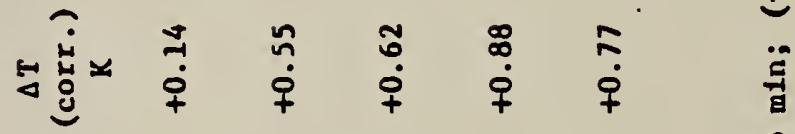

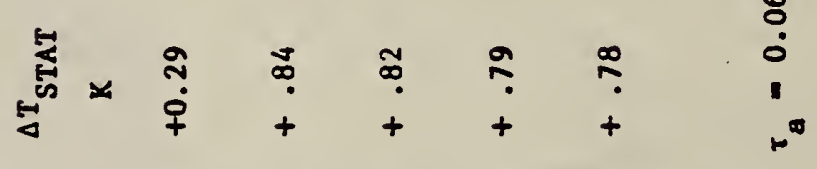

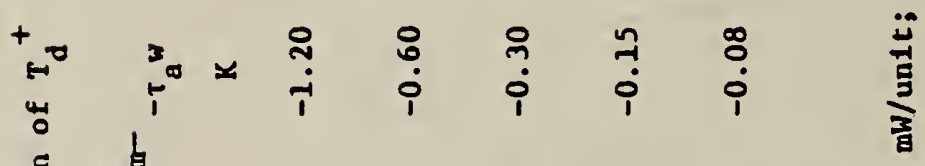

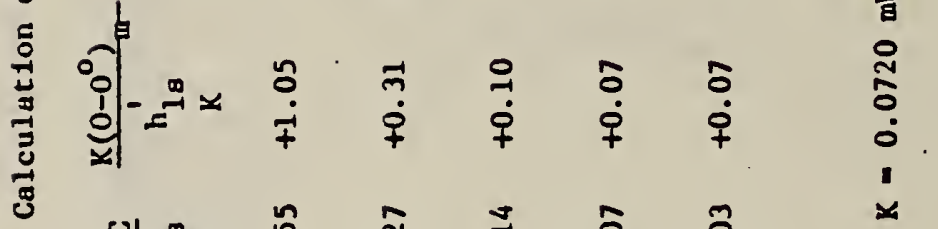

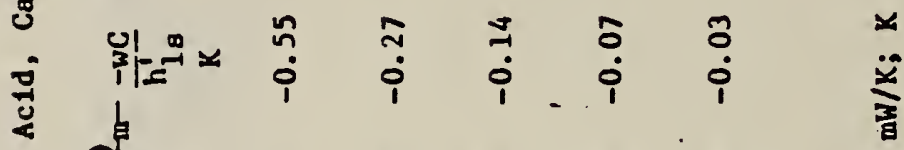

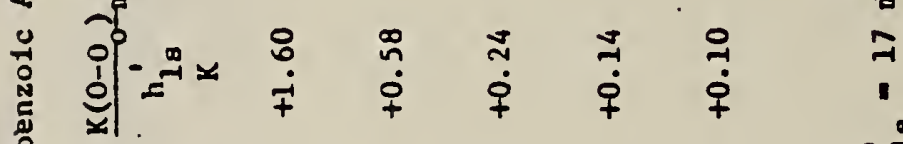

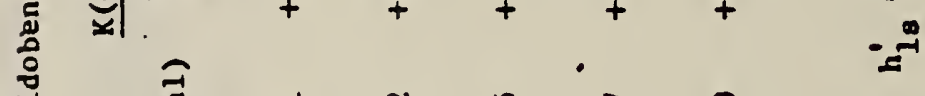

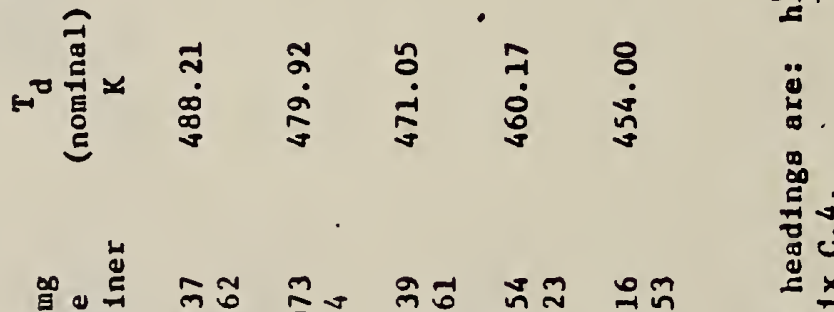

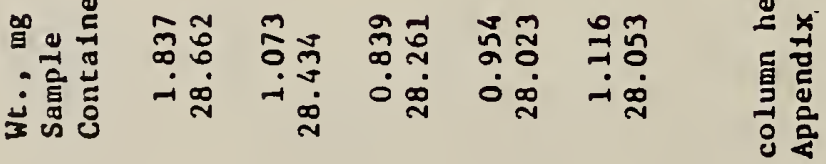

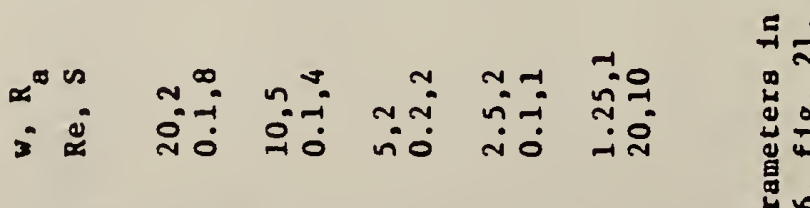

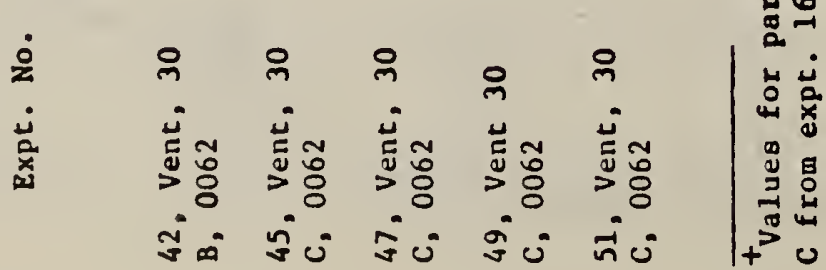




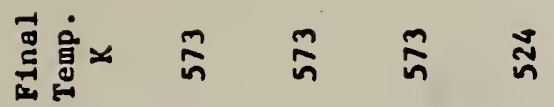

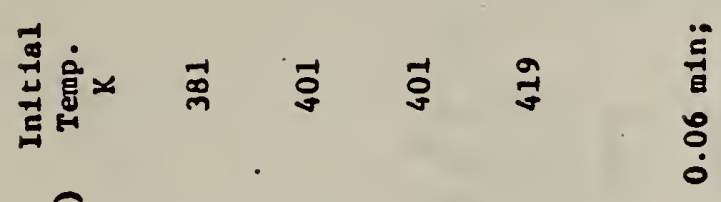

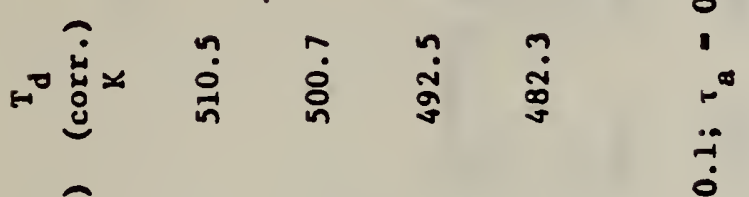

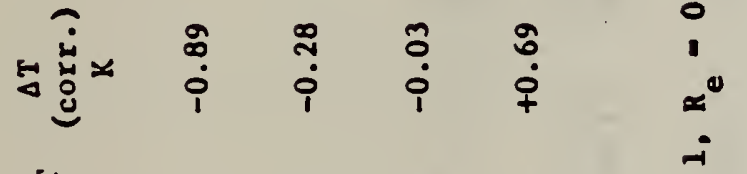

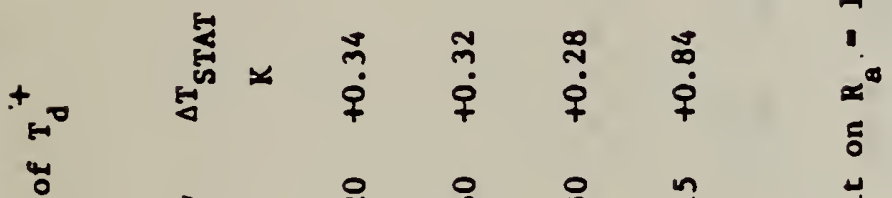

竎

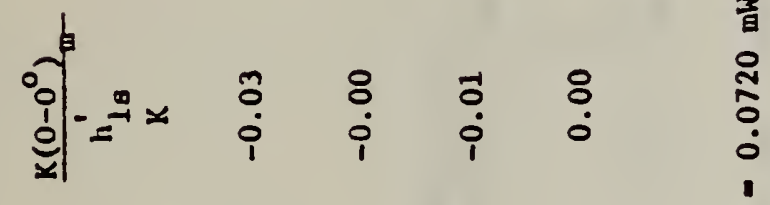

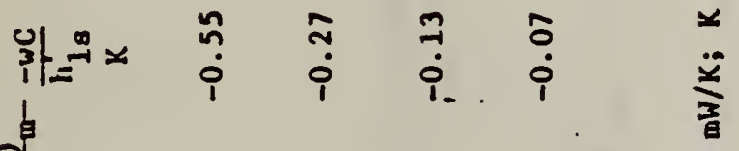

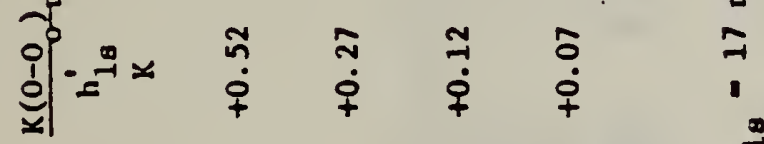

रु

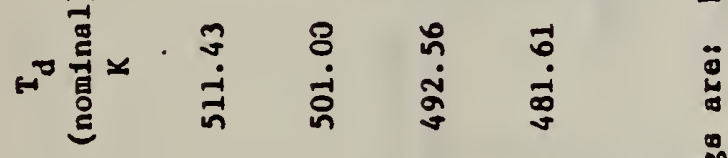

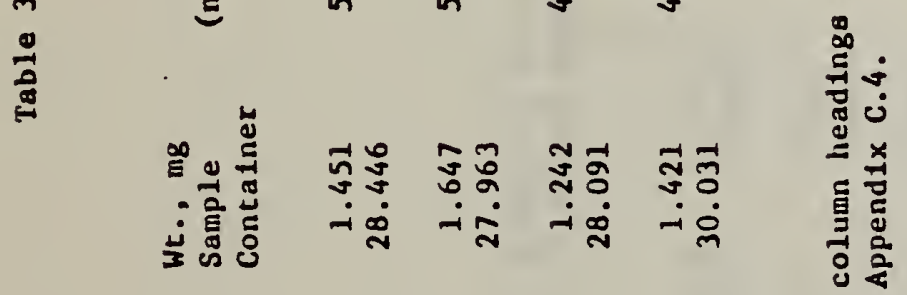

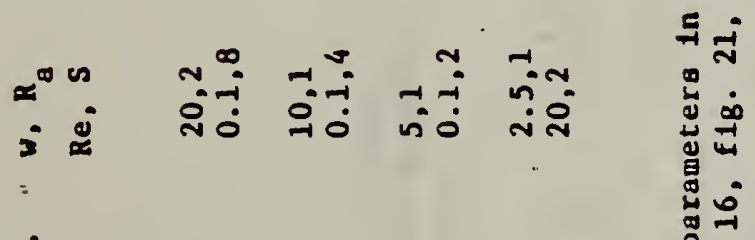

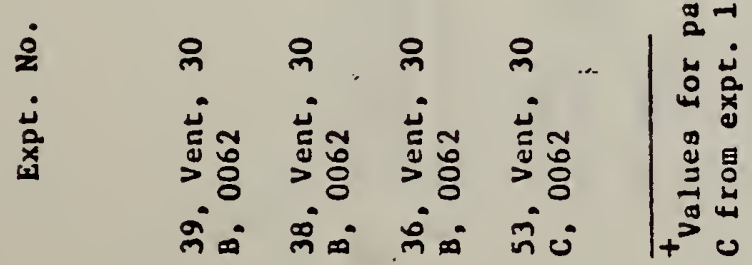




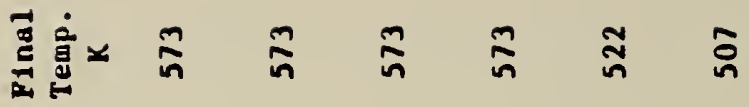

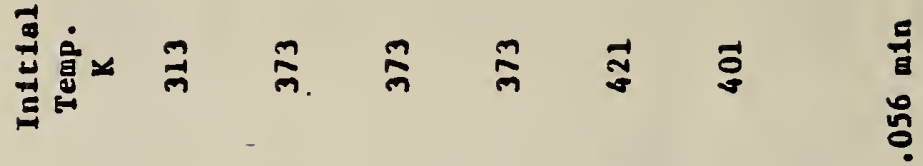

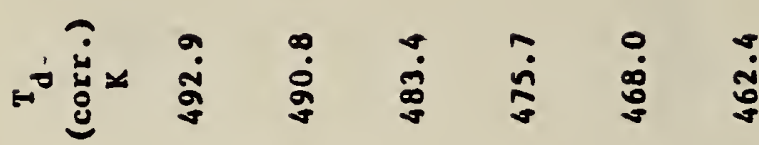

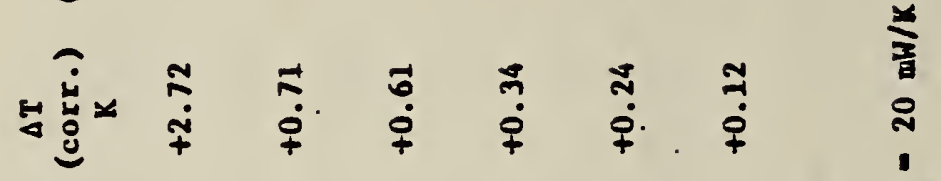

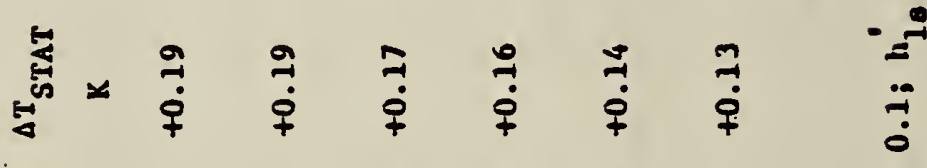

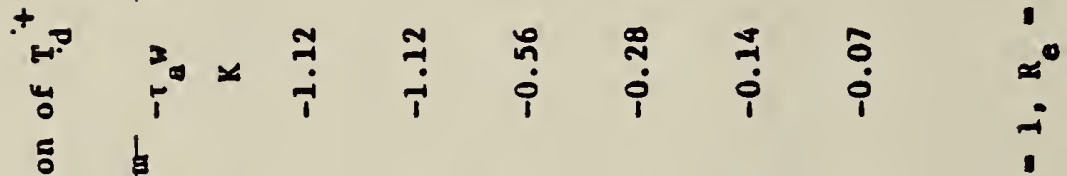

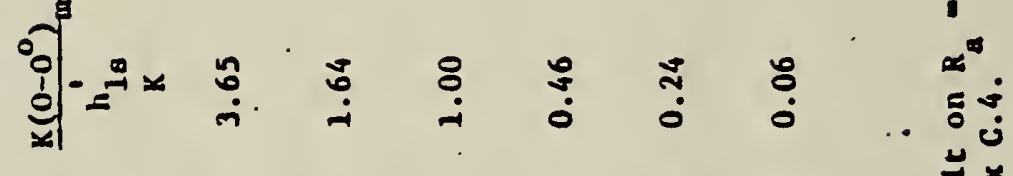

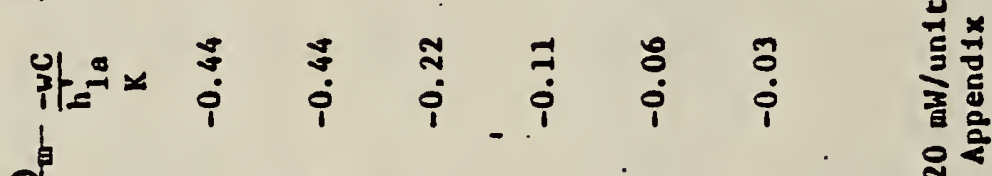

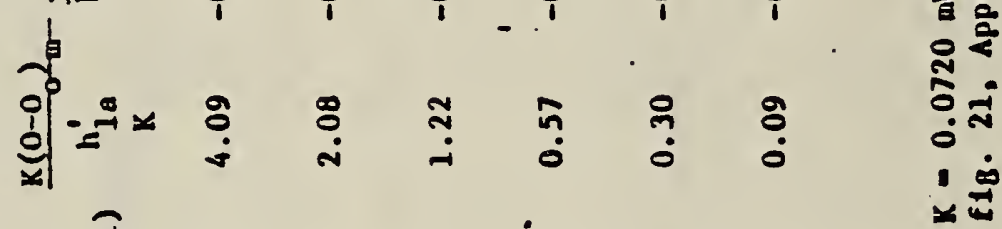

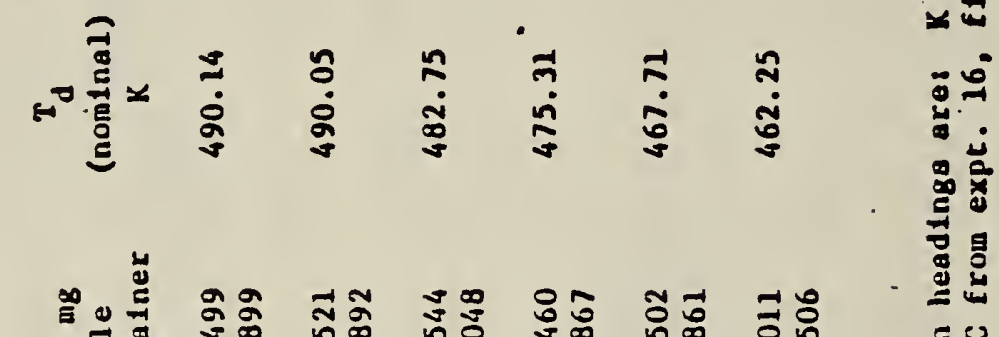

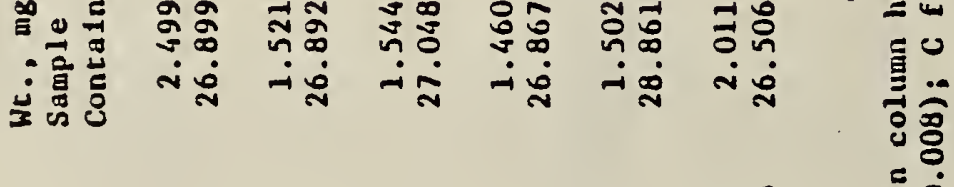

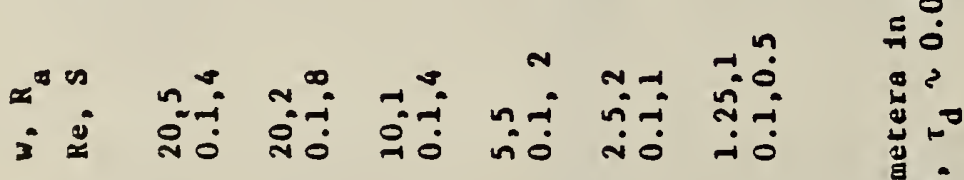

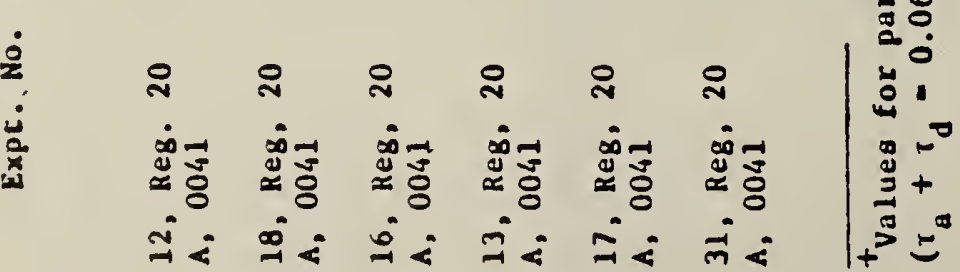


Table 5. Key to Tables 2, 3, and 4

Column

No.

1

2

3

4

5
Column

Heading

Expt. No.

$w, R_{a}, R_{e}, S$

Wt. (mg)

$\mathrm{T}_{\mathrm{d}}$ (nomina1)

$\mathrm{K}\left(0-0_{0}\right)_{\mathrm{m}} / \mathrm{h}_{1 \mathrm{~s}}$

\section{Items}

left to right, top to bottom

Experiment Number

Reg-standard block 1id; VentVented

blocklid

Inlet pressure, dry nitrogen, P.S.I.

A, B,C: $\Delta \mathrm{T}_{\text {stat }}$ from A, B, or C of fig. 19

Type of sample container (Perkin Elmer

part No.), 0062 fig. 6a; 0041, fig. 6b

w, scan rate, $\mathrm{K} \cdot \mathrm{min}^{1}$

$\mathbf{R}_{\mathrm{a}}$, sensitivity, mcals $\mathrm{s}^{-1}$

$R_{e}$, chart recorder setting, $m V$ full scale

$\mathrm{S}$, chart speed, $\mathrm{cm}^{\mathrm{min}}{ }^{-1}$

Sample weight, mg. in air

Sample container weight, ing in air

Temperature, peak of $0-0^{\circ}$. See column 5 note.

0 , observed ordinate signal at $\mathrm{T}_{\mathrm{d}}$ (nominal), decomposition run;

$0_{0}$, observed ordinate signal at $T_{d}$ (nominal), blank run with decomposition products in sample container;

$\mathrm{h}_{\text {Is }}$ heat transfer coefficient between sample container and sample holder

$\mathrm{K}$, calibration constant

$\mathrm{K}\left(\mathrm{O}-\mathrm{O}_{\mathrm{O}}\right) \mathrm{m}$, the maximum in $\mathrm{K}\left(\mathrm{O}-\mathrm{O}_{\mathrm{O}}\right)$ 
Table 5. Key to Tables 2,3 , and 4

Column

No.

6

7

8

9

11
Column

Heading

$-w C / h_{1 s}^{\prime}$

$\mathrm{K}\left(0-0^{\circ}\right) / \mathrm{h}_{1 \mathrm{~s}}^{\prime}$

$-\tau a^{w}$

${ }^{\triangle} \mathrm{T}_{\text {STAT }}$

$\Delta \mathrm{T}$ (corr.)

$\mathrm{T}_{\mathrm{d}}(\operatorname{corr}$.

Initial temp.

Final temp.

\section{Items}

left to right, top to bottom

w scan rate, $\mathrm{K} \cdot \mathrm{s}^{-1}$

$C$ heat capacity, aluminum container

$0^{\circ}$ ordinate signal at $\mathrm{T}_{\mathrm{d}}$ (nominal), blank run with no sample or container

in sample holder. Sum of columns 5,6 .

$\tau_{a}$ time lag of temperature of sample holder temperature behind program temperature.

$\Delta \mathrm{T}_{\text {stat }}$ temp. corr. of program temperature if sample holder temperature held constant

Sum of columns $7,8,9$

Sum of columns 4 and 10

Initial temperature of scanning run;

Ambient to initial at $160 \mathrm{k} / \mathrm{min}$.

Final temperature of scanning run. 
0 and $0^{\circ}$ of $\left(0-0_{0}\right)_{m}$ are computed by subtracting the isothermal base line signals from the corresponding observed signals at $\mathrm{T}_{\mathrm{d}}$ (nominal) as shown in fig. 11. The isothermal base line signals of each pair of runs at a given scanning rate were constructed by passing a straight line through the isothermal signals taken at the beginning and end of each run. The nominal program temperatures at the beginning and end of both decomposition and blank runs were the same.

$T_{d}$ (nominal) is converted to $T_{d}$ (corrected), in column 11 , which is the temperature of the sample. The formula used is

$$
\mathrm{T}_{\mathrm{d}}(\operatorname{corr})=\mathrm{T}_{\mathrm{d}}(\text { nominal })+\left(\mathrm{K}\left(0-0_{0}\right)_{\mathrm{m}}-\mathrm{wC}\right) / \mathrm{h}_{1 \mathrm{~s}}^{\prime}-\tau_{\mathrm{a}} \mathrm{w}+\Delta \mathrm{T}_{\mathrm{STAT}}
$$

This is developed in Appendix C.3.4. (see eqs. (C-22a,b). The second term on the right of eq. 16, given in column 7 with its components in columns 5 and 6 , is the temperature difference between the sample and container (assumed to be the same) and the sample holder. $\mathrm{K}\left(0-0_{0}\right)_{\mathrm{m}} / \mathrm{h}^{\prime}$ is is the correction for self heating. The determination of the energy caltbration constant, $\mathrm{K}$, is given in Table $\mathrm{C}-8$, Appendix C.4. The heat capacity, C, used here is taken from other results of expt. 16

$$
C=0.900+0.67 \times 10^{-4}\left(\mathrm{~T}_{\mathrm{p}}-380\right) \mathrm{Jg}^{-1} \text {, }
$$

which is within $5 \%$ of that of the actual containers used in the experiments (see Fig. C-7, Appendix C.4). An estimated, typical, value is used for $h_{1 s}^{\prime}$, the heat transfer coefficient between sample container and sample holder. Its uncërtainty lies between 5 and $10 \mathrm{~mW}$ and probably is closer to the former.

The term $\tau_{a} w$, in column 8 , is the time lag of the apparent mean temperature of the sample holder behind the programmed temperature. $\tau_{a}$ is from the calibration studies using melting solids, Table C-6, Apperidix 

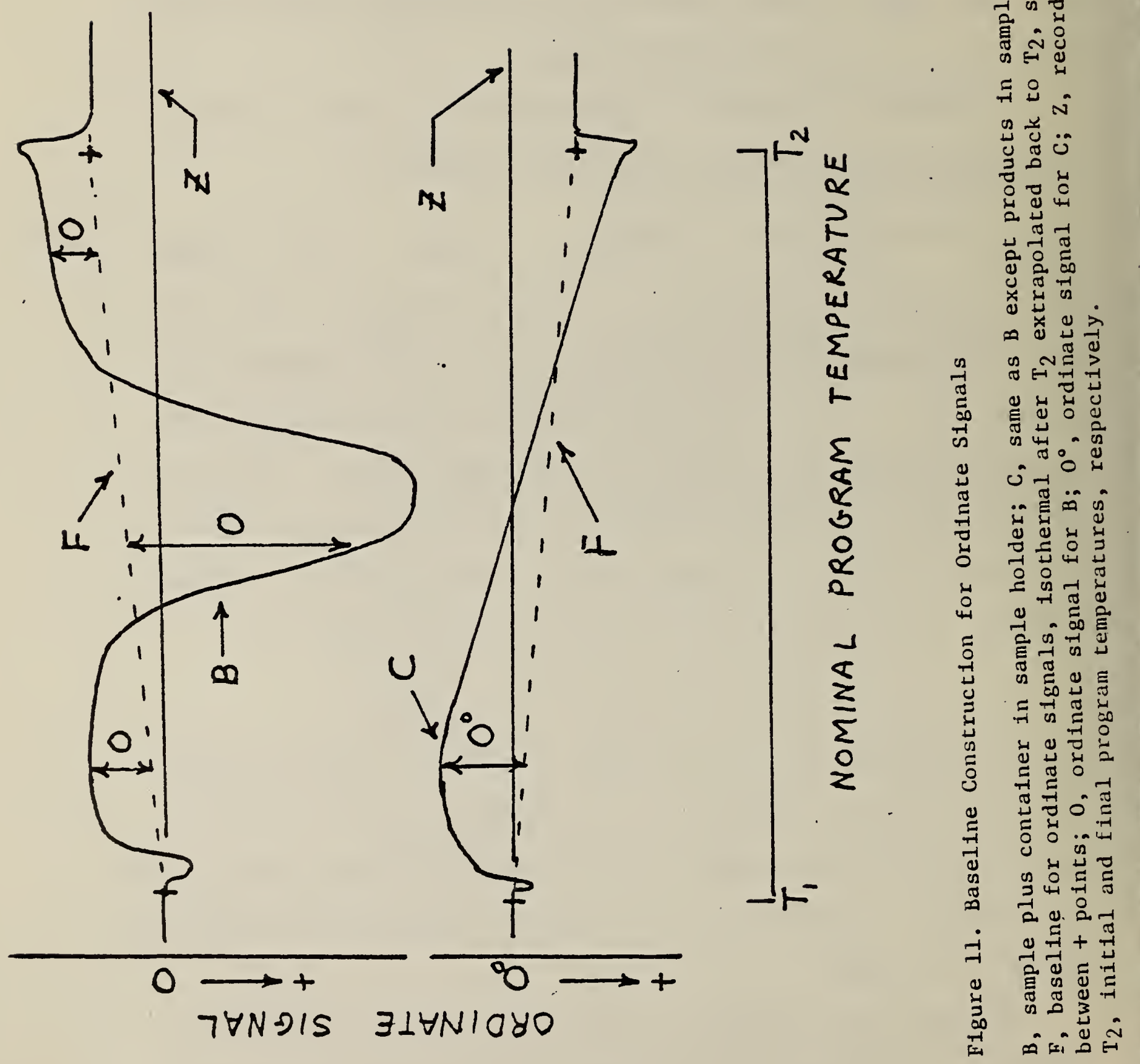
C.4. $\Delta \mathrm{T}_{\mathrm{STAT}}$, in column 9, is the correction added to the nominal programmed temperature to obtain the apparent mean temperature of the sample holder when it is held at a constant temperature. Values are from Fig. C-8, Appendix C. 4 .

It should be noted that the sum of columns $5,6,8$, and 9 , which is given in column 10, is analogous to the temperature lag described in the ASTM test so long as the product of the time constant of the output signal of the apparatus and the scanning rate, $w$, is small in comparison to column 10. It should be noted again that column 10 assumes that the maximum decomposition rate is identical to the maximum power output. The error seems to be small and is discussed in section 5.5.

Plots of the data in terms of the ASTM test method of data treatment are given in figures 12 to 14 . The length of the horizontal bar of the cross corresponds to $1 \mathrm{~K}$ (i.e. $\pm 0.5 \mathrm{~K}$ from the center). The straight line through the points was drawn visually.

The slope of the $\log _{10} \mathrm{w}$ versus $1 / \mathrm{T}_{\mathrm{d}}$ plot was determined taken from the graph and also from a least squares fit of $\log _{10} \mathrm{w}$ versus $1 / \mathrm{T}_{\mathrm{d}}$ assuming only $1 / T_{d}$ has an error. $E$ and $A$ then were calculated by the ASTM test method. To obtain some unambiguous statistics, $A$ and $E$ were calculated simultaneously by a least squares fit of the natural logarithm of both sides of equation 12 to the data (see Appendix C.5.1.). The results of these calculations are given in the computer program printout in tables 6 to 8 . Results of the three methods of calculation are summarized in table 9. An example of the variation of the results with various assumed orders of reaction is given in footnote 6 of table 9. 


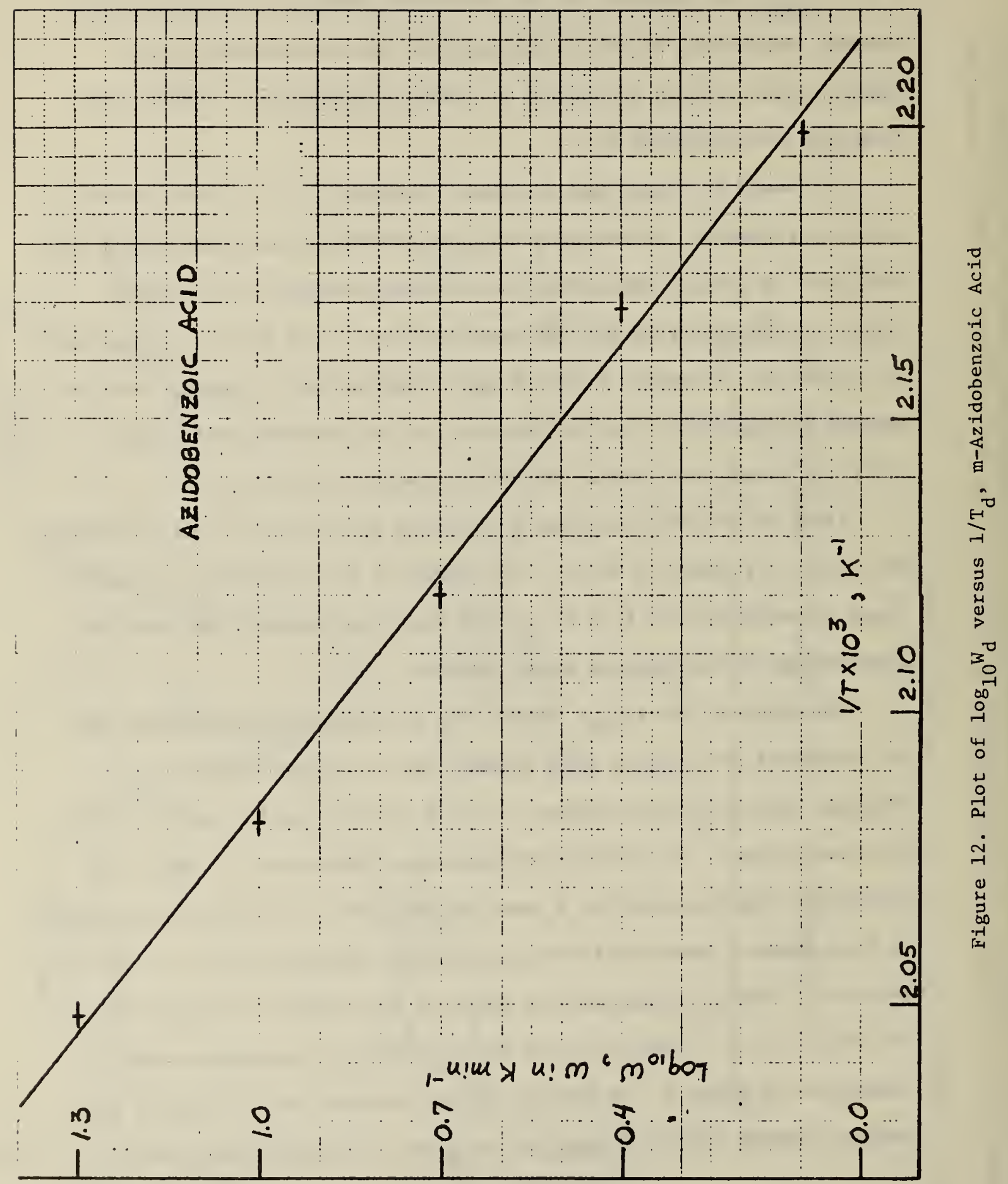




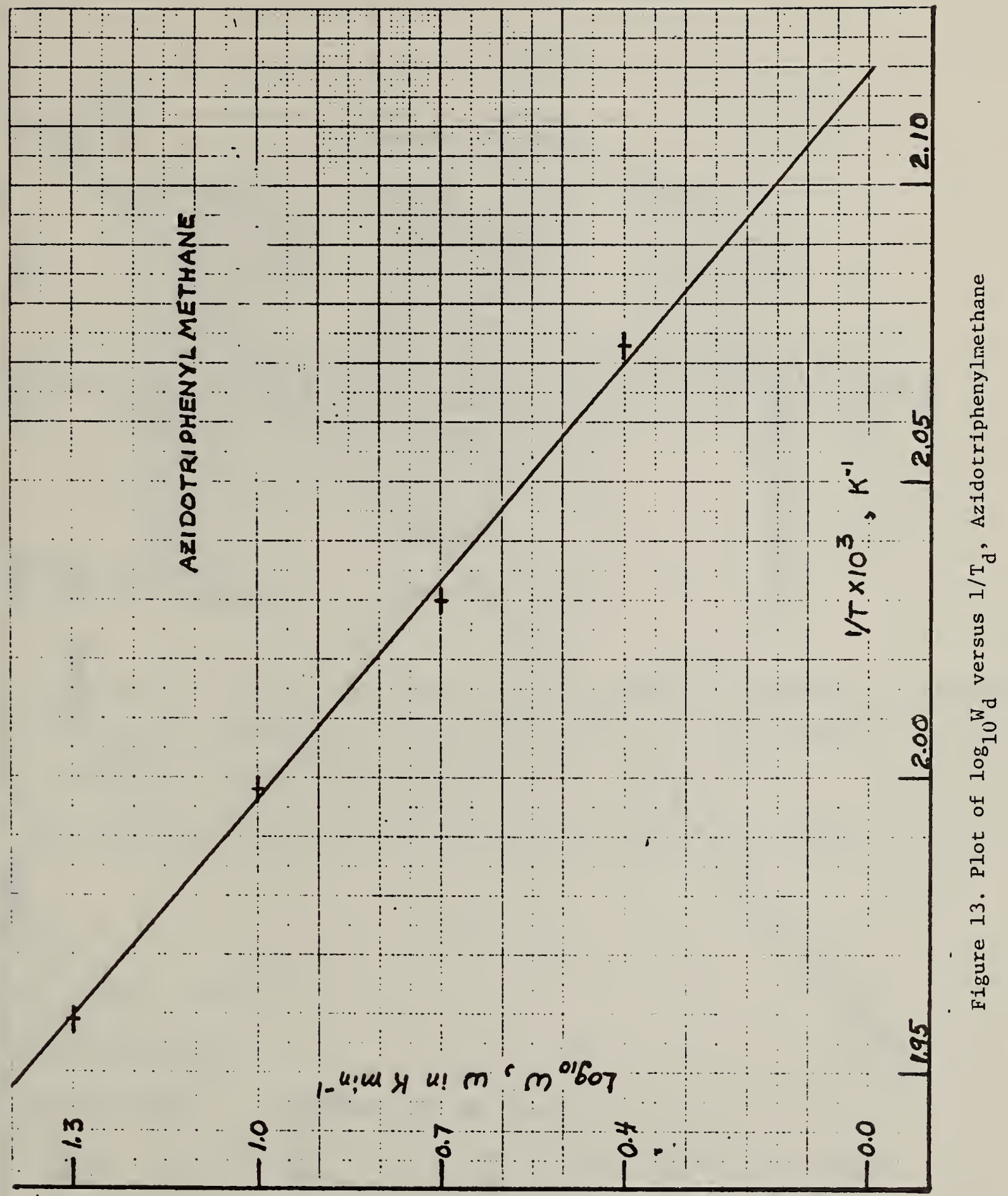




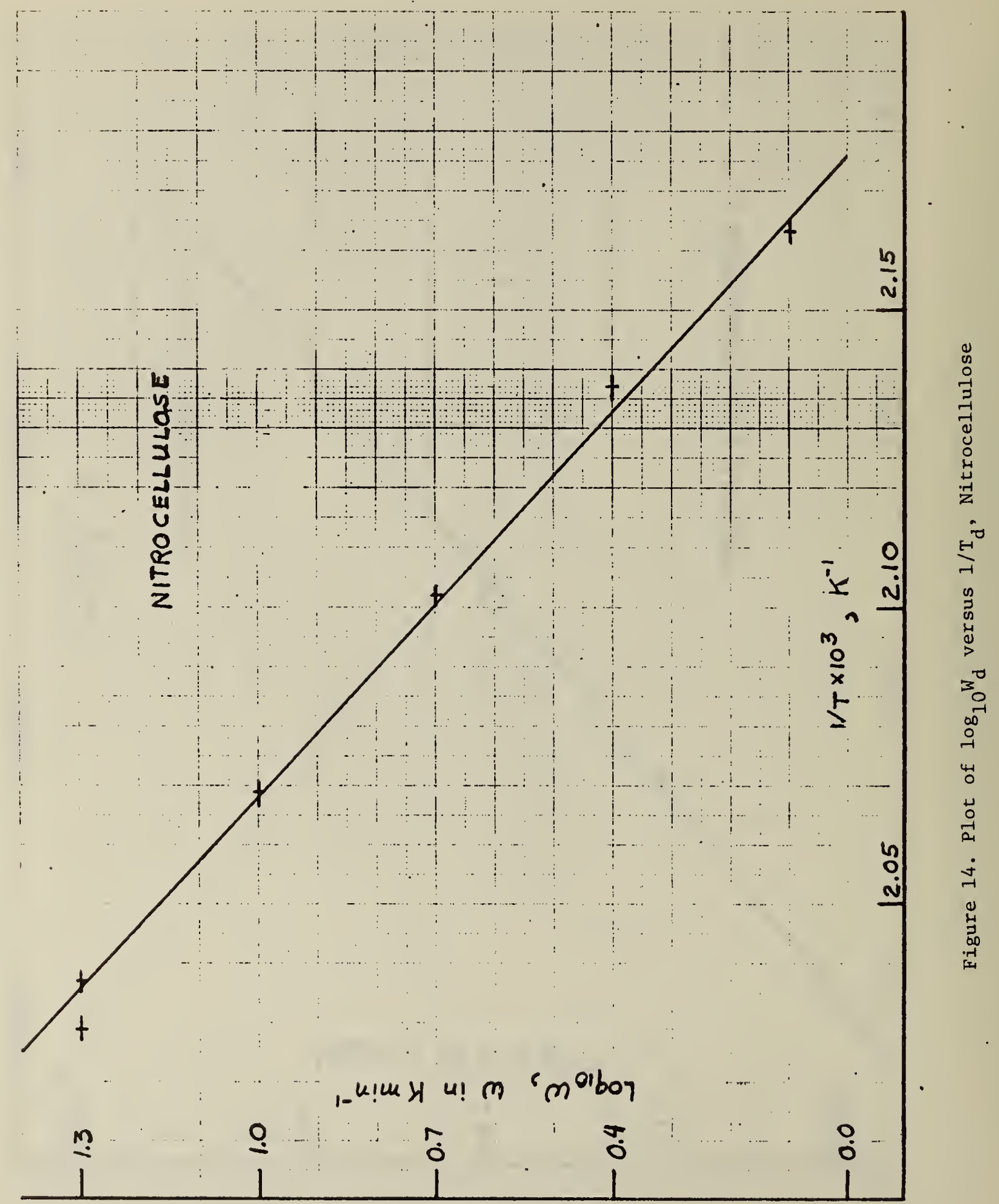




\section{Table 6. m-Azidobenzoic Acid, Kissinger Method}

KISSINIER VETHOD

NQ.DATA PAIRS 5

ITER・iNO.

1

2

3

4

$E=$

$\operatorname{LO} 3 A=$

A $1.25587 E+15$

SEAN RATE

20

18

5

2.5

1.25

VAD I ANC

$A=.22383$

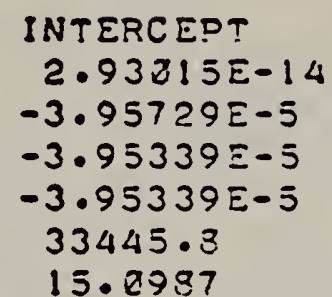

15.8987

A. MAX $5.93977 E+15$

T, OES

488.4

482.5

471.7

461.1

454.8

CIENTS

$B=.258326$
ASSUMED ORDER 1

$$
\begin{aligned}
& \text { SLOPE } \\
& 5.62738 E-2 \\
& 5.94145 E-2 \\
& 5.94146 \bar{E}-2 \\
& 5.94146 E-2 \\
& \text { STD DEV = } \\
& \text { STD DEV = }
\end{aligned}
$$

T, CZS-CAL

-. 563851.

.742452

.751143

$-1.46569$

.544325

\section{$513 * * 2$}

2. $44363 E-5$

2. $453 \therefore 2 E-5$

2. $44 \mathrm{R} 2 ? \Sigma-5$

2. 44 222E-5

1414.71

.675183

A, MIN 2.0́5121 D+14

Iter. no.: Iteration no. in least squares fit of $y^{\prime}=a-b\left[x^{\prime}+2 \ln \left(6 / y_{0}\right)-\ln (1-\phi)\right]$ to data. $y^{\prime}=y-y_{0}, y=1000 / T_{d}, y_{0}=1000(\overline{T / T})$, a and $b$, see below, $x^{\prime}=x-x_{0}$, $\mathrm{X}=\log w, \mathrm{X}_{0}=(\overline{\log w}), \phi=2(1-n)\left(\mathrm{b} / \mathrm{Y}-3(\mathrm{~b} / \mathrm{y})^{2}\right)$

Intercept: $-y_{0}+b\left(\ln a+\ln b-2 \ln y_{0}-x_{0}\right), b=1000 R / E, a=1000 \mathrm{~A}$

Slope: b

Sig $\star \star 2:$ variance

E: activation energy, cal mol ${ }^{-1}$

STD DEV: Standard deviation

log $\mathrm{A}: \log _{10^{\mathrm{A}}}, \mathrm{A}$ in $\min ^{-1}$ 
Table 7. Azidotriphenylmethane, Kissinger Method

KISSINGER METHOD

NO.DATA PAIRS 4

$1+E \square \cdot N O$.
1
2
3
4
$E=$

$\operatorname{Los} A=$

A $9.92713 E+14$

SCAN DATE

20

18

5

INTERCEPT

$1.79559 E-14$

$-2.46509 E-5$

$-2.46464 E-5$

$-2.40464 E-5$

34731.8

14.9969

ASSUMED ORDER $\mid$

A. MAX $3.78955 E+15$

T, OBS

518.4

580.6

$492 \cdot 5$

$2 \cdot 5$

$482 \cdot 3$

VARIANCE COEFFICIENTS

$A=.279212$

$B=.519192$

SLOPE

$5.41452 \Sigma-2$

$5.72140 \mathrm{E}-2$

$5.72143 E-2$

$5.72148 E-2$

STD DEV =

STD DEV =

T, OBS- TAL

$-9.44514 E-2$

-.322462

.886271

$-.469953$

$A-B=-1 \cdot 87723 \equiv-3$

\section{SIG**2}

8. $7243.3 \Xi-6$

8. $66893 E-6$

2. $61955 \equiv-5$

$8.61955 E-6$

1284.18

. 581776

A. MI is 2.5998EE+14 
Table 8. Nitrocellulose, Kissinger Method

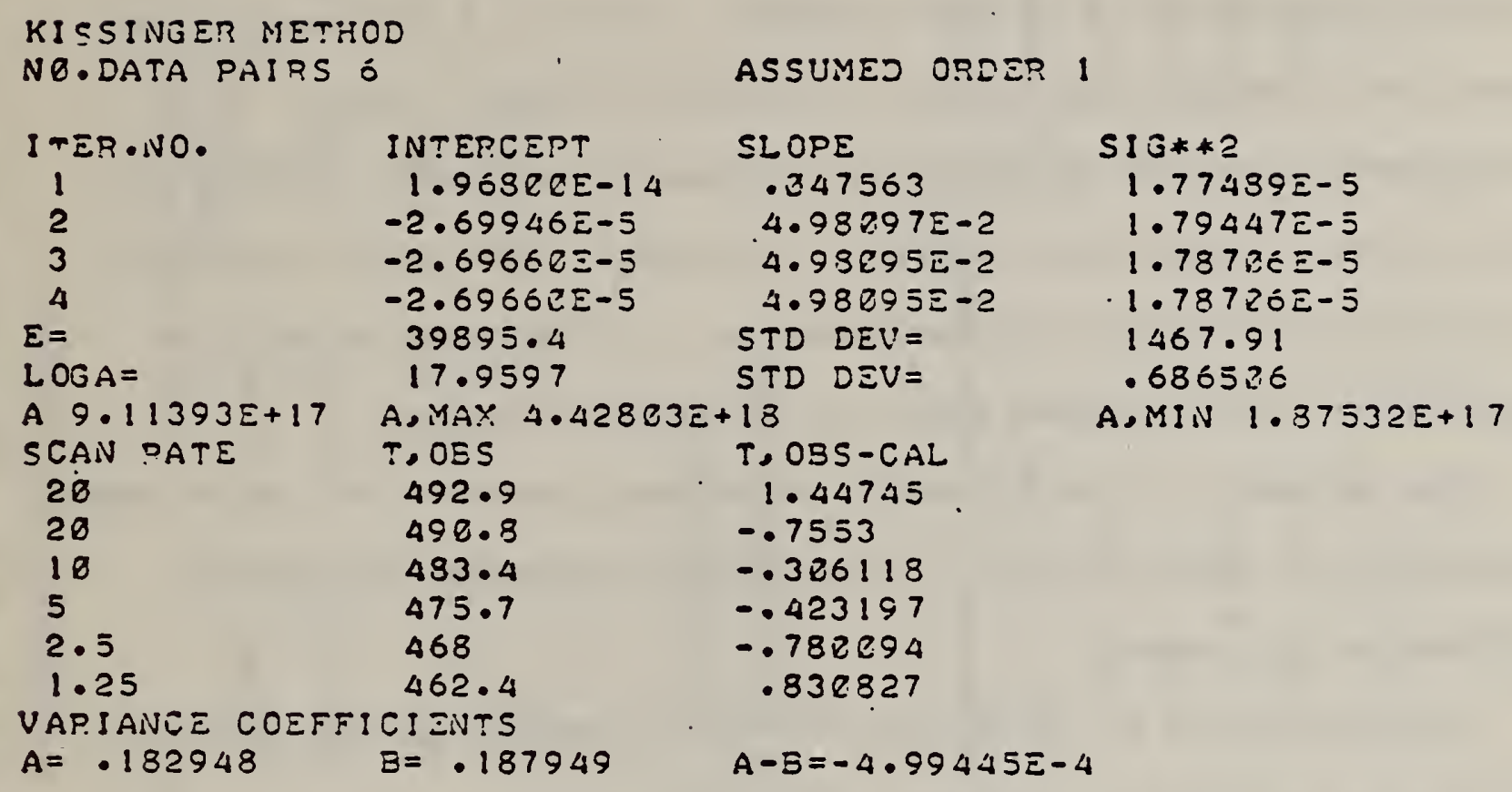


A comparison of the results on the three compounds run successfully by us is given in table 10 along with values determined by other laboratories ${ }^{+}$ using the ASTM Method ane one result for nitrocellulose plastic run by adiabatic calorimetry in an open container. Values of $\mathrm{E}$ and $\log _{10} \mathrm{~A}$ agree within their combined imprecision at the $95 \%$ confidence ${ }^{++}$level. It is also apparent that the imprecision in our results is roughly a factor of two, or more, greater than laboratory A's results. This may be associated with a number of factors discussed below but at this stage our relative inexperience with the method must also be taken into account.

The agreement of the nitrocullulose values obtained by DSC and adiabatic calorimetry is comforting but of little impact because of the possible differences in samples.

Intercomparison of the DSC and QDTA results may be made by constructing a $\log w$ vs. $1 / T_{d}$ plot for each using the parameters in Table 10 and eq. 12. For m-azidobenzoic acid, where the kinetic parameters show the largest difference, the $T_{d}$ predicted for the results of Lab $B$ agrees with ours at $w=1.25 \mathrm{k} / \mathrm{min}$, but is higher at $w=20$ (493 vs $489 \mathrm{k}$ ). This probably is acceptable for an interlaboratory comparison involving different methods. Difficulties arise, however, when the two rate constants are extrapolated. At $323 \mathrm{~K}\left(50^{\circ} \mathrm{C}\right)$, our experiments yield $\mathrm{k}=0.3 \times 10^{-7}$ $\min ^{-1}$ while those of Lab $B$ yield $k=1.6 \times 10^{-7}$ (with, of course, overlapping error bounds). This spread may be too large for establishing safe storage conditions unless a large safety factor is applied.

\footnotetext{
Talues for laboratory $A$ and $B$ were kindly made available to us by $A$. Duswalt. These values were run through our least squares program for a second validation of the program and the results are given in table 10 . They agree well with the program results given to us by A. Duswalt.

+ Standard deviations must be multiplied by $t .975$ in table 10 to obtain
imprecision.
} 


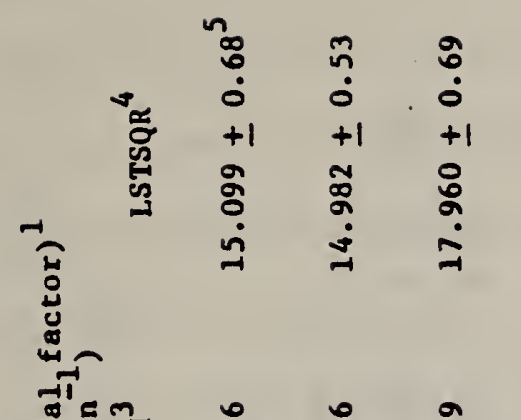

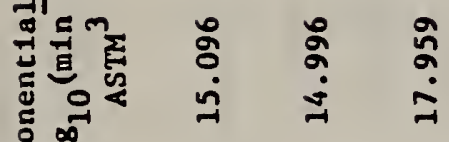
:

줄

ธ

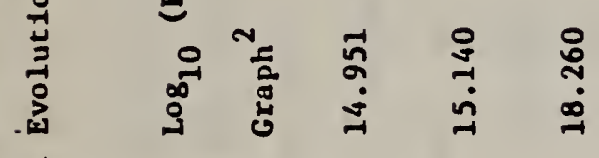

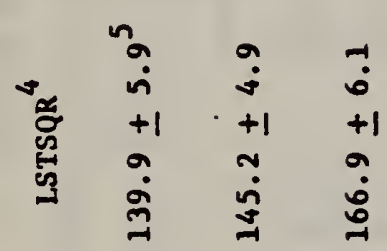

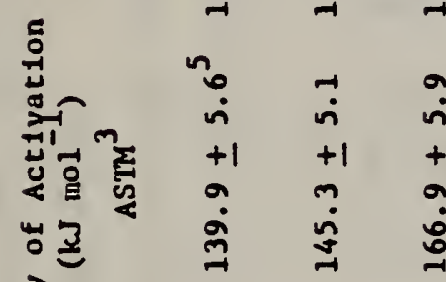

啇.

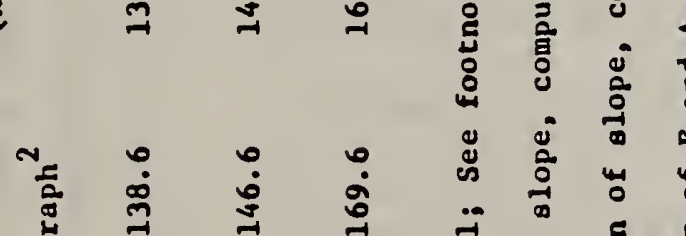

站

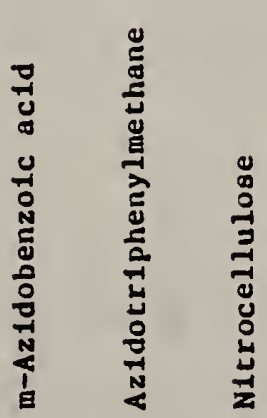

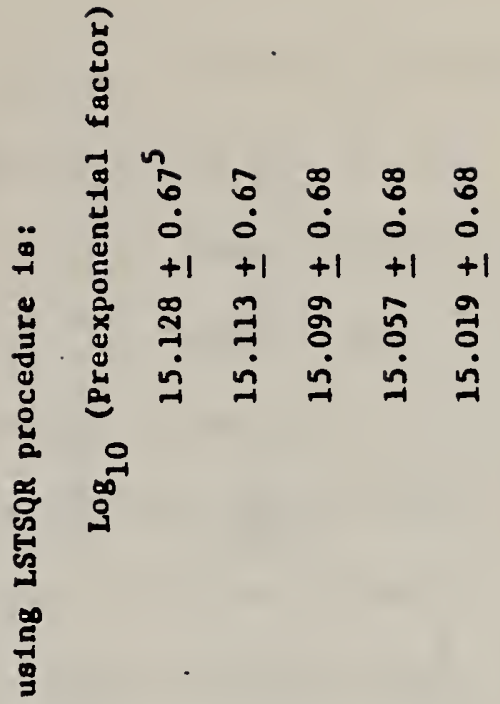

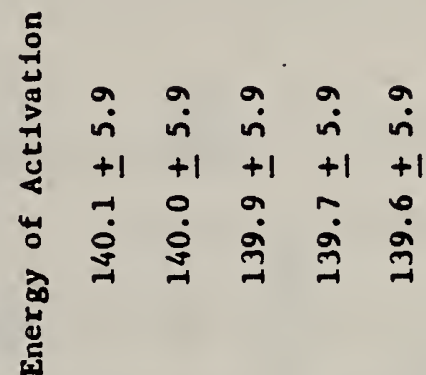

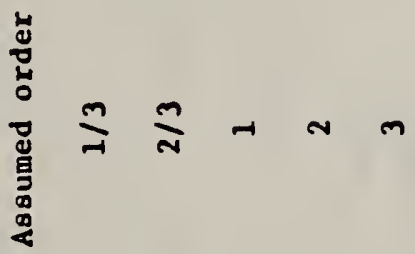




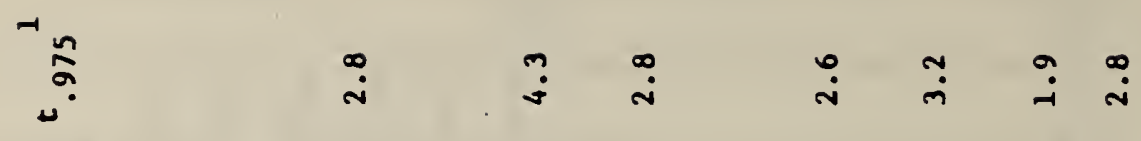

宽

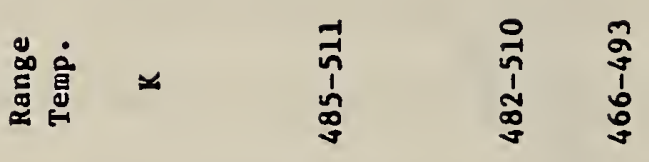

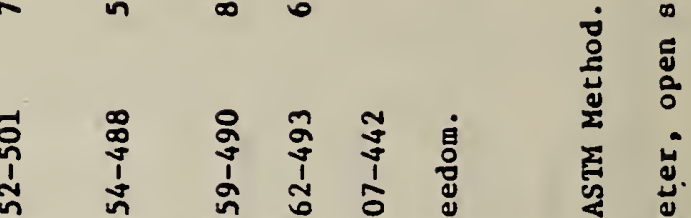

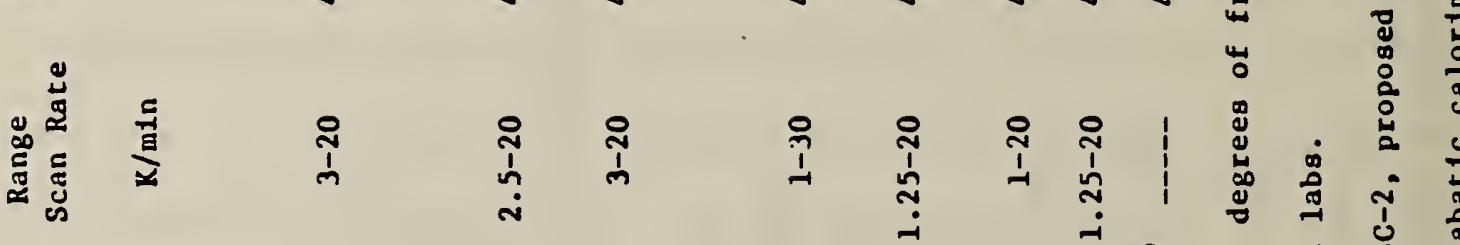

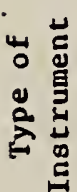

言

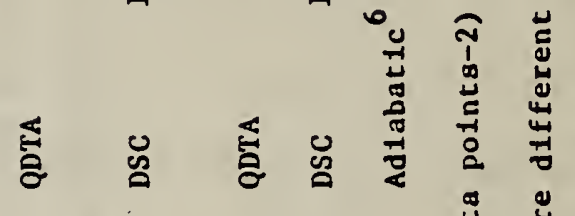

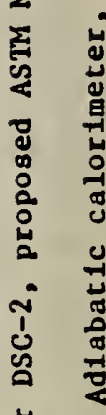

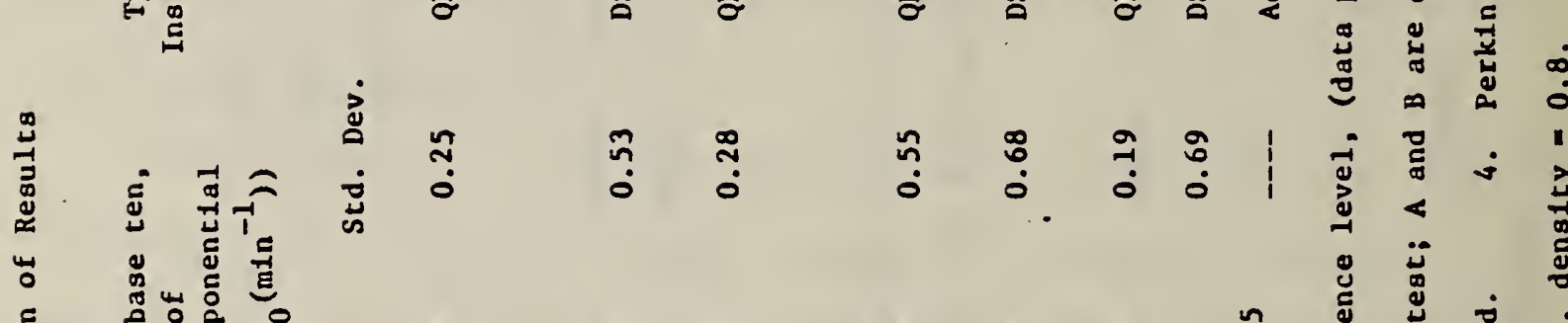

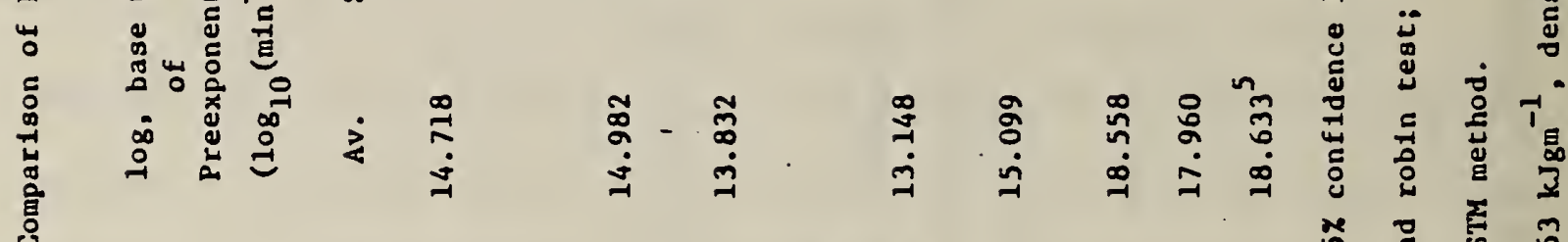

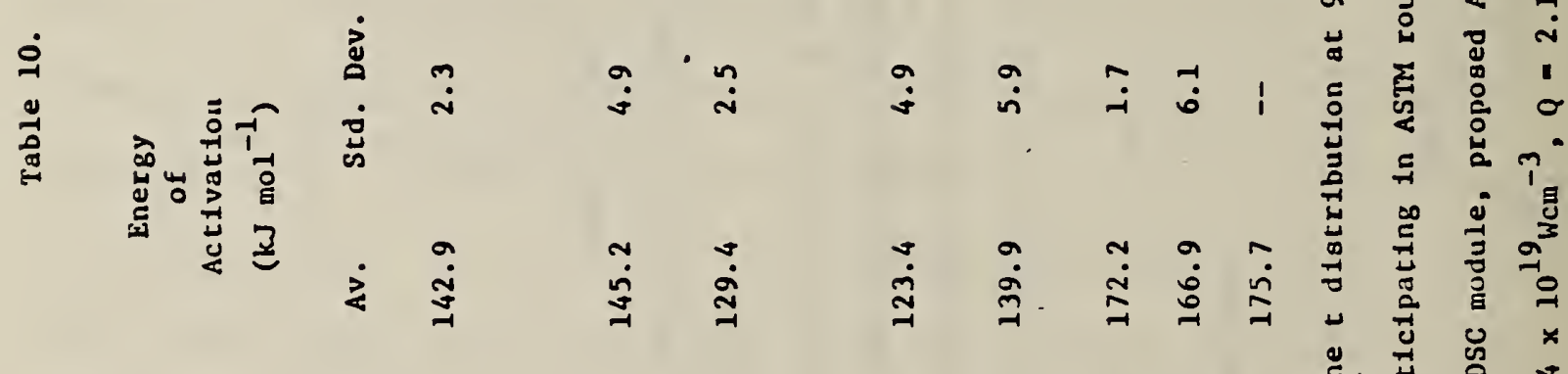

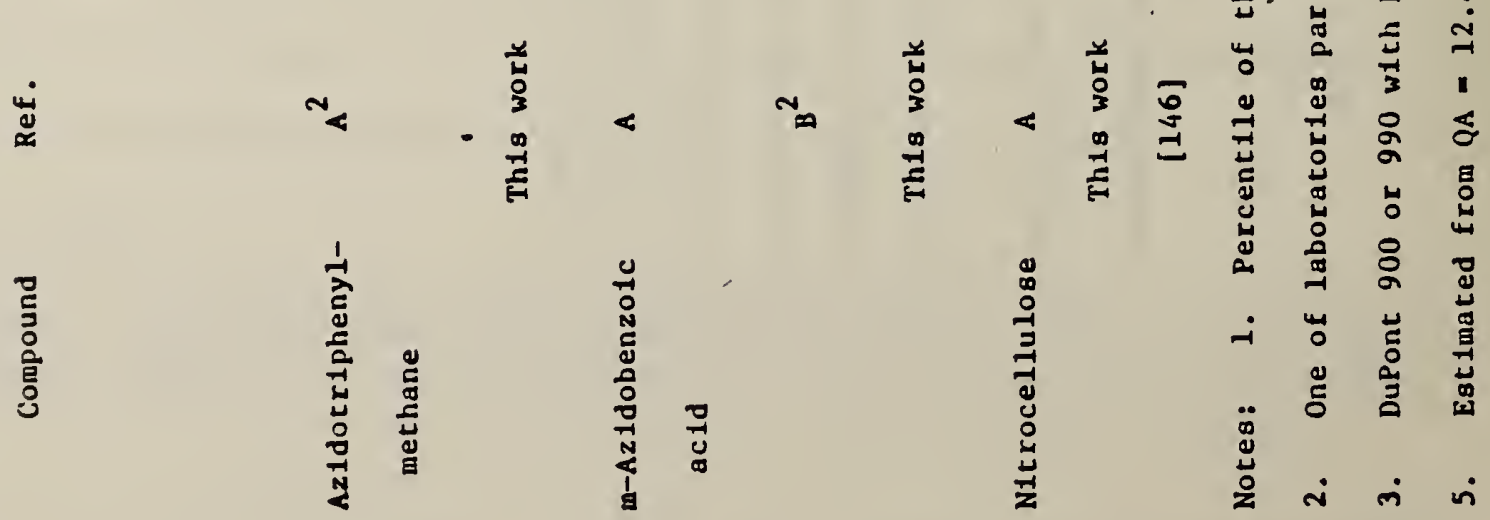


The calculation of rate parameters as a function of reaction order (footnote 6, Table 9) shows no variation. In part this is due to the Small experimental temperature range. But is is also consistent with the model. Numerical integration of eq. 9 showed that $\mathrm{T}_{\mathrm{d}}$ changes very little with reaction order and that $\eta_{d}$ has a characteristic value for each reaction order that is nearly independent of scan rate. For m-azidobenzoic acid, $\mathrm{T}_{\mathrm{d}}$ decreases about $2 \mathrm{~K}$ as $\mathrm{n}$ changes from zero to three at both $\mathrm{w}=5$ and $20 \mathrm{~K} / \mathrm{min}$. The change in $\eta_{\mathrm{d}}$ between these scan rates is less than $0.3 \%$ of its value. On the other hand, the reaction order can be determined from aging experiments at several temperatures.

We now turn to an analysis of possible systematic errors in our DSC experiments, based on the assumptions of the method. These are listed in Table 11. To our knowledge, the corrections developed below have not been applied in the QDTA studies. Thus the proper intercomparison is that in. Table 10 .

An estimate of the correction of the ordinate peak to the conversion peak, assumption 1, is worked out in Appendix C.5.3. This is

$$
T_{d}(d \eta / d t \max .)=T_{d}\left(\operatorname{cor} r_{\cdot}\right)-w\left(\tau_{s}+\tau_{d}\right)
$$

where $T_{d}(\operatorname{corr})$ is the sample temperature at $\mathrm{K}\left(0-0_{0}\right)$ m and $\tau_{s}$ is

$$
\tau_{s}=\frac{c_{1}+c_{2}}{h_{1 s}^{\prime}} \sim \frac{c_{1}}{h_{1 s}^{\prime}}=\frac{c}{h_{1 s}^{\prime}} \text { (tables 2, 3, 4). }
$$

where $C_{1}$ and $C_{2}$ the heat capacity of the sample container and sample, respectively. The second term on the right side of eq. 17 is the shift in the program temperature (the correction in the self-heating term is negligible for our experiments). For our measurements it is smaller than 
Table 11. List of Assumptions

(Proposed ASTM Test Method)

Assumptions concerning $\mathrm{T}_{\mathrm{d}}$ and $\mathrm{W}$

1.* The maximum $\mathrm{d} n / \mathrm{d} t$ occurs at $\mathrm{K}\left(0-\mathrm{O}_{\mathrm{O}}\right)_{\mathrm{m}}$

2. $d T / d t$ equals $d T_{p} / d t=w$, in eq. 12 at the maximum $d n / d t, T_{p}$ is nominal program temperature.

3. Deviations of $\mathrm{dT} / \mathrm{dt}$ from $\mathrm{dT}_{\mathrm{p}} / \mathrm{dt}=\mathrm{w}$ during entire decomposition run do not cause $g\left(\eta_{d}\right)$ to differ significiantly from a constant value of one.

Assumptions concerning temperature

4. Sample temperature is uniform.

5. Sample container temperature is uniform.

6. Sample and sample container temperatures are equal.

Assumptions concerning temperature measurements

$7 . * h_{1 s}^{\prime}$ is constant for disposable cells of same type

Assumptions concerning nature of reaction

8. Reaction is simple and obeys general rate law.

9. Reaction is not autocatalytic, partially inhibited, or does not involve melting with decomposition.

* Assumptions apparently a part of the ASTM method but neither is required for the validity of the first two equalities of eq. 12. These two equalities require one to determine $\mathrm{T}_{\mathrm{d}}$ and $(\mathrm{dT} / \mathrm{d} t)_{\mathrm{d}}$. 
the measurement error since the time constant of the sample and its container is small (i.e. $\tau_{s}=\mathrm{C} / \mathrm{h}_{1 \mathrm{~s}}^{\prime}$ of column 6 of tables 2,3 , and 4) and the time constant of the ordinate signal detection (or measurement) system is also small (i.e. $\tau_{d} \leq 0.01 \mathrm{~min}$, Appendix C.4). The changes in $E$ and $A$ based on the estimate of eq. 17 are given in table 12. E, A and $\mathrm{K}$ all increase slightly, but are still within the measurement error. The error. would be far from negligible for a sample container of large heat capacity and a reasonably accurate correction would have to be worked out. '

The error in assumption 2, the rate of change of sample temperature being equal to the programmed rate (at the maximum) is small so long as the maximum in $d \eta / d t$ and the DTA peak nearly coincide. The magnitude of this error is worked out in Appendix C.5.3 (see eqs. C-47, 48). It is

$$
\left(\frac{d T}{d t}\right)_{d}=w\left(1+\frac{2 a_{2}}{h_{1 s}} \frac{w C}{h_{1 s}}\right)
$$

where $a_{2}$ is the curvature of the ordinate signal in $\mathrm{wK}^{-2}$ at $\mathrm{K}\left(0-\mathrm{O}_{0}\right)_{\mathrm{m}}$. In appendix C.5.3 it is also shown that the magnitude of $(\mathrm{dT} / \mathrm{dt})_{\mathrm{d}}$ at $\mathrm{K}\left(0-\mathrm{O}_{\mathrm{o}}\right)_{\mathrm{m}}$ is

$$
\left(\frac{d T}{d t}\right)_{d}=w\left(1+\frac{2 a_{2}}{h_{1 s}^{\prime}} w \tau_{d}\right)
$$

Thus, to be consistent the values of $T_{d}$ (corr) of tables 2,3 , and 4 should be used with eq. 19 if we assume the maximum in the ordinate signal corresponds to the maximum in $\mathrm{dn} / \mathrm{dt}$ and eqs. (17) and (18) should be used to calculace $T_{d}$ and $(d T / d t)_{d}$ at the maximum $d n / d t$.

The values of E, A, for nitrocellulose from table 10, the ASTM method, are given in the first row of part $b$ of table 13. The values in the second row are also for the ASTM method but without expt. number 12, which 


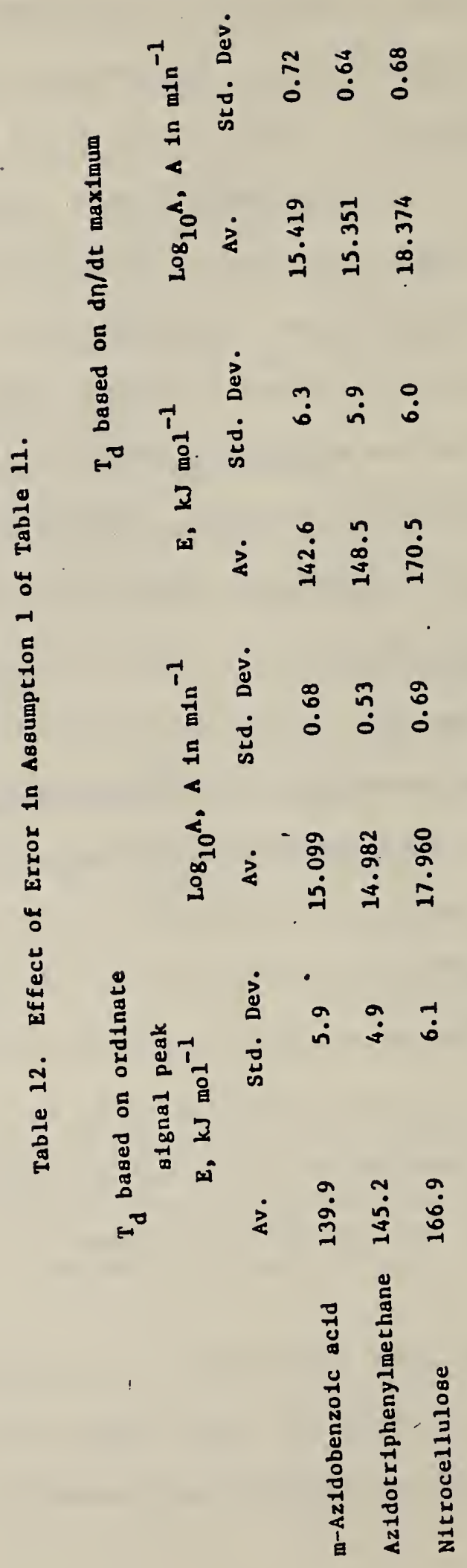


Table 13. Effect of Error in Assumption 2 of Table 11.

(Nitrocellulose)

(a) Correction for scanning rate

Experiment

18

16

13

17

31

\section{$\underline{\mathrm{w}, \mathrm{K} / \mathrm{min}}$}

$\underline{\text { nominal }}$

20

10

5

2.5

1.25

(b) Changes in rate parameters

\footnotetext{
$E, \mathrm{~kJ}$ mol $1^{-1}$

$(d T / d t)$

Value std. dev.

$\log _{10^{A}} A$ in $\min ^{-1}$

T

value std. dev.

$1_{\max }$ Ord.

w

166.9

6.1

17.960

0.69

2 max Ord.

w

173.1

4.6

18.668

0.52

$\max$ 0rd.

eq. 19

173.2

4.6

18.672

0.52

$3_{\max } d \eta / d t$

eq. 18

181.0

6.3

19.565

0.7

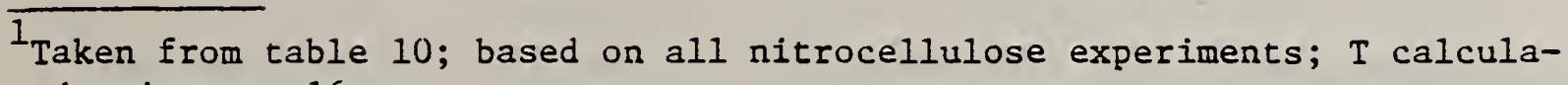
ted using eq. 16 .

${ }^{2}$ Nitrocellulose expt. 12 not included in calculation of this and lower rows. T calculated using eq. 16.

$3_{\mathrm{T}}$ calculated from the sum of eq. 16 and eq. 17.
} 
has a large self heating correction. The values of the second row are the "base" for the error estimate. Experiment 12 was not used in the other calculations. The values of $\mathrm{E}$ and $\mathrm{A}$ in the third row are for both $\mathrm{dT} / \mathrm{dt}$ and $T$ of the sample at the maximum ordinate signal. The values in the fourth row are for both $\mathrm{dT} / \mathrm{dt}$ and $\mathrm{T}$ of the sample at the maximum $\mathrm{dn} / \mathrm{dt}$. The estimated correction for $\mathrm{E}$ and $\mathrm{A}$ for these five experiments is less than their standard deviations. As for assumption 1, the corrections for assumption 2 increases $\mathrm{E}, \mathrm{A}$ and $\mathrm{K}$.

Assumption 3, that $\mathrm{dT} / \mathrm{dt}$ for the sample is equal to the programmed temperature scan rate, w, throughout the exotherm cannot be met exactly. The increment to the scanning rate due to self heating can be derived from the exotherm and is its derivative with respect to time. It shows the characteristic maximum and minimum of the derivative of a "line" with finite half-width. The effect of self heating on $g\left(\eta_{d}\right)$ is considered in Appendix C.5.2 where it is shown to be order dependent and vanishes for a first order reaction. An upper bound is derived. The sign of the correction is indicated by the bound

$$
E=\frac{E(\text { apparent })}{1+\varepsilon(n-1)}
$$

where

$$
\varepsilon<\frac{E}{\mathrm{RT}_{\mathrm{d}}^{2}} \frac{\mathrm{K}\left(\mathrm{O}-\mathrm{O}_{\mathrm{O}}\right)_{\mathrm{m}}}{\mathrm{h}_{1 \mathrm{~s}}^{\prime}}
$$

For a reaction order $\mathrm{n}>1, \mathrm{E}$ decreases due to the effect of self heating on the constancy of $g\left(\eta_{d}\right)$. This is opposite in sign to the assumption that $g\left(\eta_{d}\right)$ is constant for the rate law if $n \neq 1$ and $d T / d t$ for the sample equals w. For activation energies such as are found in our experiment, 
the error in $E$ will be less than $2 \%$ if the temperature excursion is kept below $0.2 \mathrm{~K}$. (This is the repeatability desired for the ASTM method.)

This upper bound estimate may be too conservative to be useful and must be improved by numerical calculations. Some of our experiments had larger temperature excursions and they increased with $\mathrm{T}_{\mathrm{d}}$.

Assumption 4, uniform sample temperature, is crucial for the theory of the method. Fortunately, the error associated with it seems to be negligible in our experiments. This error is estimated below.

Using nitrocellulose as an example, one can estimate the critical explosion temperature $T_{*}$, in our apparatus for a $1.5 \mathrm{mg}$ sample using the measured heat of decomposition, $2.19 \mathrm{~kJ} \mathrm{gm}^{-1}$, a heat transfer coefficient of $20 \mathrm{~mW} / \mathrm{K}, E$ of $167 \mathrm{~kJ} \mathrm{~mol} 1^{-1}$, and $\log _{10^{A}} \mathrm{~min}^{-1}$ of 17.96 . One obtains a value of $491 \mathrm{~K}$. This yields a ratio of $0.301 \mathrm{~s} / \mathrm{K}$ for $\Omega / \mathrm{w}$ where $\Omega$ is the dimensionless scanning rate defined in eq. (B-64) of section B. 8 . Assuming sample and sample container are in much better thermal contact with each other than the container is with the sample holder, one has for $\gamma_{*}$ (defined in eq. B-67) a value of 20.1. From ref. [165], fig. 5, one can see that if $w$ is $20 \mathrm{Kmin}^{-1}$, the ratio $\left|\Delta \theta_{\mathrm{m}}\right| /\left|\Delta \theta_{q}\right|$, corresponding to $\gamma_{*}$ of 0.1 and $\Omega$ of 0.1 , is of the order of 2 to 2.3 from eq. (13) of that reference. This ratio is $\Delta \mathrm{T}_{\mathrm{m}} / \Delta \mathrm{T}_{\text {int }}$ where $\Delta \mathrm{T}_{\text {int }}$ is defined in eq. $\mathrm{B}-68 \mathrm{~b}$ of section B.8. $\Delta \mathrm{T}_{\mathrm{m}}$ is the maximum difference in temperature between the center and surface of the sample during the decomposition run: Since $\Delta \mathrm{T}_{\text {int }}$ is independent of Biot number and fig. 5 of [165] applies to infinite Biot number, the aforementioned ratio provides an upper bound on $\Delta \mathrm{T}_{\mathrm{m}}$ so long as. one is willing to approximate the reaction as being homogenous and assume heat transfer is solely by conduction in the sample during the decomposition. 
Assuming the sample is in the form of a thin sandwich, $g_{2}=1$ in eq. B$68 \mathrm{~b}$, and estimating the thermal diffusivity from data in ref. [146] table $k$, as $0.0018 \mathrm{~cm}^{2} \mathrm{~s}^{-1}, \Delta \mathrm{T}_{\text {int }}$ is $-93 \mathrm{r} 2 \mathrm{Kelvin}$ for $\mathrm{w}$ equal to $20 \mathrm{~K} / \mathrm{min}$ and a half thickness of the nitrocellulose film of $\mathrm{r}$ in $\mathrm{cm} . \Delta \mathrm{T}_{\text {int }}$ is $0.004 \mathrm{~K}$ if, for example, one assumes $\mathrm{I}$ is $0.006 \mathrm{~cm}$ and thus $\Delta \mathrm{T}_{\mathrm{m}}$ is of the order of $0.01 \mathrm{~K}$. Since $\Delta \mathrm{T}_{\mathrm{m}}$ is proportional to $\mathrm{w}$, one can see by analogy with the results of table 12 (i.e. the change in $\mathrm{T}_{\mathrm{d}}$ was $0.64 \mathrm{~K}$ at an $w$ of $20 \mathrm{~K} / \mathrm{min}$ that the error for the example cited would be totally negligible.

It is not clear, at present how accurate the above analysis is so it must be regarded as tentative ${ }^{+}$. One should note that the estimated error is small because our experiments were carried out with small sample thickness and not because the sample weight is small. The estimated effective Biot number is also quite small. For the example we are using, the effective Biot number, Bi, can be estimated as

$$
B 1=\frac{\alpha_{0} r}{\lambda}=\frac{h_{1 s^{\prime}}^{\prime}}{A \lambda}=\frac{h_{1 s^{\prime}}^{\prime} r^{2} \rho}{m \lambda} \sim 7
$$

A is. the area of the sample which can be expressed in terms of its density (taken as 0.82 from ref. [186]), $\rho$, its mass $m(.0015 \mathrm{~g})$ and an assumed thickness $r^{2}\left(.0126 \mathrm{~cm}\right.$, the actual thickness of the film). $\mathrm{h}_{1 \mathrm{~s}}^{\prime}$ is the heat transfer coefficient $(.02 \mathrm{~W} / \mathrm{K})$ between the sample container and holder.

\footnotetext{
For example, the dimensionless critical scanning rate, assuming the decomposition is a first order reaction is 1.3 from the numerical result cited in Appendix B.8. Accordingly, for the example given, the critical value of $w$ would be $\sim 260 \mathrm{~K} / \mathrm{min}$ ! In principle a thermal explosion should not occur for this example situation in our instrument! Until this and other consequences of theory are examined experimentally, one must treat the calculations with caution.
} 
Qualitatively, the error analysis associated with assumption 4 confirms the proposed ASTM procedure for preparing samples. The analysis also suggests that one should also run the experiment with smaller values of $\mathrm{h}^{\prime} \mathrm{s}^{\circ}$. There is a limit to the effectiveness of this procedure, as discussed in Appendix C.1, because thermal loading of the sample container produces temperature gradients in the sample even if $h^{\prime}{ }_{1 s}$ is zero.

For small containers made of materials of large thermal diffusivity and conductivity, assumption 5, uniform sample container temperature, will contribute little error. Assumption 6, equal sample and container temperatures, is crucial for the validity of and must hold for the entire decomposition experiment. This would be important even if the sample temperature were measured directly. In addition it implies that the gaseous decomposition products escape quasistatically and at a temperature equal to the container. It implies that very good thermal contact is maintained between container and sample throughout the decomposition. Probably none of our experiments fulfilled this condition adequately. The error in meeting assumption 6 is urknown.

The error associated with assumption 7 , the same heat transfer coefficient for all similar cells, can be appreciable but it seems probable that procedures can be developed to eliminate it. One possibility is to place the sample container on a smooth lightweight platform made from a material of high thermal conductivity. If the heat transfer coefficient between the platform and sample holder is, for example, 0.1 of the coefficient between the platform and container, variations in the net coefficient and holder will be 20.1 of the variation in the coefficient between the platform and container. In effect, the platform is a thermal shunt. 
Another possibility is that the heat transfer coefficient can be determined via a time constant analysis of the transient in starting a scanning run with and without the sample container. It has the drawback that one must have a reliable and sensitive method of separating out the effect of the time constant of average power circuit $(3.6 \mathrm{sec}$ ) as well as that of the difference power circuit $(\approx 0.5 \mathrm{sec})$. The possibility that a method of data analysis can be devised may be possible by application or extension of the analysis of Appendix C.4.

- Procedures are available for determining if the reaction is simple (assumption 8) and, if so, determining $f(\eta)$ of eq. 11. These are discussed in ref. [165], section III. Procedures for "separating" complex reactions under certain circumstances are discussed in ref. [152] as well as procedures for determining the kinetics of decomposition outside the temperature range of the actual observed exotherms. The general problem of obtaining heat evolution kinetic data for systems that melt with decomposition has been studied by Rodgers [207] and Dorko, et al [208211].

In summary, the systematic error analysis indicates that the errors in the kinetic equation used to analyze the data in the ASTM method are or can be arranged to be kept small provided the simple general rate law, eq. 8;, applies. Also at least some techniques are available and probably more (or better ones) could be developed to determine when eq. 8 does not apply. The systematic errors associated with carrying out kinetic measurements by calorimetry of any type are more difficult to assess. We conclude (some what to our surprise) that there is some reason, albeit theoretical, that the sample temperature is probably uniform in our experi- 
ments. Also it is possible that an experimental technique to detect nonuniformity of the sample temperature can be devised. However, efforts to assess the error in the assumption that the sample and sample container temperatures are equal have not been successful. While it seems reasonable to conclude that one can avoid this assumption by measuring the temperature of the sample, this is, in a sense, the same assumption in disguise. The assumption to be analyzed is then the equality of the temperature detector and sample.

It is difficult to see how the necessarily limited temperature range of the data obtained by this method will ever provide sufficiently precise values of $\mathrm{E}$ and $\mathrm{A}$ to permit reliable extrapolation to lower temperatures even if the decomposition mechanism does not change. While there are ageing techniques that permit measurements at lower temperatures, they are indirect and time consuming. 


\subsection{Conclusions Based on the Experiments and Recommendations for Future}

Work

1. Intercomparison of DSC and QDTA results with those from other methods are needed. Emphasis should be on studies at lower temperatures.

The low sensitivity of commercial DSC and QDTA instruments makes them usable only at relatively high temperatures. The exothermic reaction at high temperatures may not be the dominant heat producer at the lower temperatures characteristic of shipment and storage. That is, the mechanism may change with temperature.

One protection against this possibility is to build into the proposed ASTM test method the lower temperature aging tests used by Duswalt [24] They extend the range of validity of the scanning method results. They could be extended to even lower temperatures, but would become very time consuming. An alternative approach is desirable.

A second question is whether or not procedures that use very small samples can mimic bulk hazard conditions. The DSC and QDTA tests use a system consisting of a thin layer of material sandwiched between a metal container and cover radding appreciable heat capacity. Correlation of the results on such systems with bulk samples requires that the properties of the material itself be extracted and then interpreted using thermal balance equations. The validity of this approach is more in question for marginally unstable materials than for explosives, for which see Rodgers et al $[23,149]$.

Because of these questions the DSC and QDTA methodology should be tested against heat conduction and adiabatic calorimetry using larger samples. Lower temperatures can be used in the latter two methods; how 
high temperature data should be extrapolated can be explored. Predictions of explosion temperatures from all four types of experiments can be compared using methods developed by Bowes $[119,120]$.

Heat conduction calorimetry is included in the proposed comparisons because of its flexibility. Both isothermal and programmed studies can be made, including non-linear programming, which may have advantages. Also, the sample is in relatively poor thermal contact with its environment (i.e., is quasi-adiabatic) which should promote uniformity of temperature, an important goal that is discussed later.

It cannot be ruled out that these intercomparisons would show that testing of marginally unstable materials should be based on the explosion temperature test, although that is time consuming and highly empirical.

2. Provision should be made for the use of both open and closed containers in DSC and QDTA tests for thermal instabilicy hazards.

Closed container tests can indicate maximum hazard conditions: both with respect to pressure rise and catalysis by gaseous products. Ideally, a measure of the pressure rise should be incorporated, because this is an important hazard parameter $[148,150,155,156]$. But this will require substantial development. In the absence of a pressure measurement two types of closed containers can be used. One is the reusable high pressure cell discussed briefly in section 5.4. The other is a high implosion but low burst pressure cell. This could be used in an apparatus in which the sample holder can be pressurized (e.g. DuPont DSC apparatus).

Studies with open containers (e.g. [104,212]) are needed to verify hazards associated with catalysis by products and to determine whether or not vaporization of material (absorbing heat) mitigates the hazard. 
There are problems in experiments with open containers that must be studied. First, good thermal contact must be maintained. Second, the gases should be lost nearly quasistatically if all of the liberated heat is to be measured. The perforated lid in contact with the material may be suitable for controlling these features. Third, condensation of products outside the sample container can lead to changes in instrument sensitivity as observed in our work. (See discussion of emisivity effects in section 5.4.) Here the preferred solution is to flush gaseous.products out of the system during the experiment, instead of introducing the assumption that emissivity effects are proportional to the extent of reaction $[213]$

3. The sample temperature in a DSC or. QDTA experiment must be controiled to match the assumptions of the method. This means, that the sample and its container should be at the same temperature and that gradients within the former should be minimized.

Identity of sample and container temperatures is required in the model and would be necessary even if the temperature of the sample were measured directly. The control circuitry responds to and the output circuitry records the history of the combination. A spring-loaded lid (perforated to allow escape of gases in an open container experiment, e.g. a screen [214]) should be effective for solids, even when there is appreciable change in volume during the reaction. Introduction of an inert liquid film between sample and container might be an improvement $[215,216]$. Increased surface area for heat transfer (perhaps fins on the lid) could be used for gaseous reactions. Finally, direct measurement of the temperature difference between sample and container should be attempted, although the small size of samples used in DSC and QDTA experiments make this difficult. Any of these variants must be tested experimentally before being adopted for a test procedure. 
Uniformity of temperature within the sample is necessary because the temperature is measured indirectly and it must be associated with a property of the material that is sensitive to temperature. Temperature gradients in the samples used in our experiments probably were unimportant. They can be reduced by decreasing the efficiency. of heat transfer between sample container and its holder relative to that between the sample and its container.

There are limitations on this approach, as discussed in Appendix C.1 for adiabatic calorimetry. Thermal loading due to the heat capacity of the container can introduce gradients even when the heat transfer between sample and container is high. The use of thin samples counteracts the effect, as may be seen in the experiments of Merzhanov [165]. Partial thermal isolation of the sample container may enhance the possibility of thermal runaway. This would influence the choice of scanning rates (which should be less than the critical value). The optimum arrangement must be found experimentally using tests with various known heat transfer efficiencies.

4. More detailed data analysis is desirable. This would assure that the measurements fall within the limitations prescribed for the method.

The proposed ASTM test uses only the peak temperature for the decomposition and the nominal scanning rate. Analysis of the exotherm can yield $Q$, the rate $1 \mathrm{aw}$ and heat transfer data. The actual scanning rate for the sample can be obtained. Possibly, simultaneous analysis of all runs in a series can provide information on the functional form of $k(T)$ [165]. These data are all of interest for the interpretation of aging experiments. 
Efficient application of more detailed analysis will require digital data logging and computer processing if the extended method is to be more than a research tool.

5. Improved accuracy and precision would result from measurement of the heat transfer coefficient between the actual sample container used and the sample holder.

The present practice is to use an average heat transfer coefficient for disposable containers based on ones drawn from the batch used in the experiments. We conjecture that the heat transfer of a container can be determined either by a thermal shunt or from analysis of the starting transients in a scanning run, but these must be tested. 
A. Remarks on the first NBS Appraisal of Methods for Estimating

\section{Self-Reaction Hazards}

\section{A.1. CHETAH Program}

A precise summary of the CHETAH program is given in ASTM Publication DS51 [3]. The material on pp. 1, 6-9, and 96-115 were found to be particularly relevant in the review of the first NBS study [1].

The purpose of the program is to provide a quick, low-cost, screening technique to identify materials that may be a potential explosive hazard ([3], p. 6) prior to further laboratory testing ([3], p. 8). The program -was not intended to be the sole test of whether a chemical is hazardous ([3], p. 8). The procedures for estimating thermodynamic parameters (solely from chemical composition) that are used in evaluating the explosive hazard were put into the program in order to have some means of screening the estimated 100,000 new chemicals encountered each year in research and development in the chemical industry prior to measurement of their thermochemical properties.

In the original CHETAH scheme, a material that is an explosive hazard is one which is shock sensitive. The latter is defined to mean the material decomposes, with or without detonation, either upon impact by a falling weight, explosion of a No. 9 blasting cap, or a $50 \mathrm{~g}$ pellet of tetry 1 ([3], p. 99). A combination of the first three of the four empirical criteria used to estimate the degree of explosive hazard was capable of identifying all 83 shock sensitive compounds in a group of 218 compounds. However, roughly $12 \%$ of the 135 insensitive materials were categorized as being shock sensitive. The CHETAH program and other evaluation schemes are still undergoing development (see [3], p. 101 for proposed future work and [25] for an example of this work). 
CHETAH was developed by some of the members of ASTM Committee E-27. This committee also is concerned with standardization of nomenclature and tests for the energy hazard potential of chemicals. This hazard is defined as the degree of susceptibility to ignition or release of energy under environmental conditions (see [3], p. 96, and [2]). This definition is equivalent to the preliminary definition of thermal instability adopted in the first NBS study. There the hazard potential is called explosive sensitivity. The latter term is used in work dealing with explosives (see [10], p. 1-8). The three main empirical criteria used in the evaluation of the degree of the energy hazard potential in CHETAH are based on the (maximum) enthalpy of decomposition, enthalpy of combustion minus enthalpy of decomposition, and the oxygen balance. Unfortunately, these parameters are not directly related to explosive sensitivity or the energy hazard potential. Evidence in support of this conclusion is as follows.

For explosives, it seems well established that the enthalpy of decomposition per gram of material is primarily an indicator of the damage potential of an explosion, called explosive power (see [10], p. 5-24) and is only indirectly related to explosive sensitivity. Examples in support of this conclusion are the distinction between primary and secondary high explosives (see [10], p. 1-4; [26], pp. 801-802; [27], p. 4.9-15), the direct relation of the heat of decomposition with explosive power (see [10], Chp. 3; [26]), and the indirect effect of enthalpy of decomposition on impact sensitivity implied in current theories of impact sensitivity (see [10], section 12-3).

For explosives, it also seems clear that oxygen balance is a measure of explosive power. Oxygen balance correlates with ballistic mortar 
test results and enthalpy of decomposition [28]. Price indicated in 1955 [26] that the ballistic mortar test is only a qualitative measure of explosive power. However, the more recent discussion of reference 4 , section 3-9, indicates the maximum work measured in the ballistic mortar test correlates well with the enthalpy of detonation for partially confined charges determined calorimetrically. Evidently, the test determines the maximum work for explosives with near zero oxygen balance with the least error (see [10], p. 3-3).

Thus, although the parameters used in the hazard criteria in CHETAH are not directly related to sensitivity, the empirical criteria themselves are indicators of explosive sensitivity, simply because they succeed in identifying sensitivity to.shock.

For the purpose of setting regulations, the first NBS study concluded that this distinction, or apparent inconsistency, is important because it meant that there is no mechanism (definitions, criteria, etc.) for identifying materials that are shock insenstive but thermally unstable. Thus, it appeared that CHETAH could not serve as a screening method for what is assumed to constitute much of the class of materials of interest (i.e. borderline hazardous materials). This topic was explored further in the second study [8]. 


\section{A. 2. STULL-CRUISE Program and NFPA Hazard Index}

Stull's first publication on the scheme [5], deals only with the correlation of thermodynamic parameters with the NFPA hazard index [27]. In ref. [5], it was found that the parameter $\left(x_{0}-x_{d}\right) / X_{d}$ correlates well with the NFPA hazard index. The subscript o refers to oxidation and $d$ to decomposition. The quantity $X$ was either enthalpy, maximum temperature ${ }^{+}$, or maximum pressure $e^{+}$. A plot of $x_{0}-X_{d}$ versus $x_{d}$ can be divided into sectors consisting of materials having the same NEPA rating for about $80 \%$ of the total (50) compounds considered. The agreement between the sorting of materials when X was enthalpy, temerature, or pressure was close (i.e. about 95\%). The preference of temperature for $X$ seems to be based on the fact that the values of $T_{0}-T_{d}$ versus $T_{d}$ came very close to forming single straight line for the compounds considered. It seems useful to note that apart from the fact that $\Delta H_{d}\left(\right.$ or $T_{d}, P_{d}$ ) is computed from products determined by a modified equilibrium method in the CRUISE program, the parameter $\left(\Delta \mathrm{H}_{\mathrm{o}}-\Delta \mathrm{H}_{\mathrm{d}}\right) / \Delta \mathrm{H}_{\mathrm{d}}$ is the parameter used in the second criteria of the CHETAH program.

The NFPA reactivity ranking scheme is essentially a qualitative hazard index appropriate to fire fighting conditions. It contains both sensitivity to explosion and explosion power criteria required for any realistic hazard index. "Explosive power" seems to be associated primarily with the violence of the reaction of the material with water and the words "detonation" and "explosive" are used in describing a material as susceptible to explosive decomposition or reaction. "Explosive sensitivity", in this scheme appears to be related to strength of thermal or mechanical

\footnotetext{
+Under adiabatic conditions at constant volume.
} 
stimulus and the associated ambient temperature-pressure conditions required to initiate the decomposition (be it explosive or otherwise). On this basis, one would judge the differences between categories 0,1 , and 2 and the differences between categories 3 and 4 to involve differences in sensitivity to explosion by self-reaction. The difference between categories 2 and 3 would clearly involve differences in explosive power due to self-reaction. A useful introudction to the NFPA as well as other consensus hazard ranking schemes related to self-reaction hazards is given in reference [25a]. 


\section{A.3. Summary of the Results of the First NBS Assessment}

The validity of the hazard rankings predicted by the two predictive schemes (CHETAH and STULL-CRUISE) was first assessed by comparing predictions with explosion sensitivity assigned on the basis of experience for groups of compounds (e.g. primary explosives or detonators, secondary explosives, materials that can self-polymerize, and relatively stable compounds). In a more detailed assessment, the numerical values of the three different molecular parameters involved in computing the degree of hazard in the predictive schemes were compared with sensitivity test data for a group of known explosives. The three molecular parameters were the experimental values for the enthalpy of decomposition, oxygen balance (i.e. defined by the composition of the material), and the activation energy of decomposition as estimated from bond dissociation energies. Each of these parameters was separately correlated with the sensitivity data from impact, thermal surge, and explosion temperature tests. This was done because the different test methods gave somewhat different sensitivity rankings for the compounds.

The results of these two assessments were as follows:

(1) The CHETAH predictive schemes overrank the explosive sensitivity of compounds that experience indicates are "safe" and both predictive schemes do not clearly identify a polymerization hazard, as such. Both of these two findings were verified in more extensive comparisons in the second DOT study (see [8] p. 69-97). Both findings are in accord with how the CHETAH hazard criteria were devised (see section A.1.), and the second finding would be consistent with the fact that neither scheme automatically'(i.e. without operator intervention) considers polymeric decomposition products. 
(2) Comparison of the reaction products produced by either predictive scheme with available experimental data showed reasonable agreement for organic explosives but poor agreement (either in species or amounts) with data or experience for non-explosives and polymerization reactions. The products predicted for non-explosives lead to more negative enthalpies of decomposition and this accounts for part of the tendency towards too high a hazard ranking for "safe" materials. A preliminary examination of some alternative rules for predicting decomposition products that produce better agreement with experiment indicated some improvements might be possible. However, there is no simple substitute for experimental decomposition data.

(3) The sensitivity test data for explosives showed a rough correlation with activation energy, some correlation with oxygen balance, and essentially no correlation with enthalpy of decomposition. The relatively clear-cut correlation of the data with activation energy was found to be consistent with what one might expect from thermal ignition theory. That is, the greater the decomposition rate constant, the greater the material sensitivity becomes. Since the preexponential factor was relatively constant for the compounds studied, the sensitivity should increase as the activation energy decreases. The absence of a clear correlation between sensitivity data and the enthalpy of decomposition is in accord with the fact that latter is primarily a measure of explosive power. That the oxygen balance showed some correlation with the sensitivity data was unexpected. A possible reason for this correlation was that, for the compounds examined, the overall rate constant decreases as the amount of oxygen increases because the relative amounts of unsaturated hydrocarbons 


\section{A.4 Recent Developments}

Treweek et al [25a] have addressed the problem of providing the user with a single predictor of sensitivity as opposed to the four indicators of the CHETAH program. They have introduced the concept of the "interpreter" by means of which the several indicators are combined. The technique used is an application of pattern recognition, in which a series of independent parameters are correlated against some class variable, here impact sensitivity or reactivity. The interpreter does not provide a detailed relative ranking but instead places the materials in broad classes, such as non-sensitive and sensitive. The interpreter concept is very pertinent to the regulatory problem of decoding, broadly, how various chemicals should be handled.

Two types of interpreters have been developed. The first correlates sensitivity to impact with the four CHETAH parameters plus one additional parameter, - the number of peroxide bonds in the molecule. Peroxides are a class that is not easily characterized by CHETAH. Two classes are used: non-sensitive and sensitive. The second interpreter correlates the NFPA reactivity rating and the similar U.S. Coast Guard self-reactivity ratings with the four CHETAH Parameters and two other for olefinic monomers - enthalpy of polymerization and a bond dissociation energy difference. Three classes are developed: non hazardous, hazardous decomposition or polymerization, and explosive. Both interpreters are stated to be applicable to organic compounds with three or more carbon atoms.

The performance of the interpreters is very good in the test cases presented. For the impact sensitivity interpreter, 105 compounds were assigned correctly to their classes and four were over estimated. 
Using this interpreter 127 other compounds, for which the impact tests are considered are less certain, were assigned correctly.

The self-reactivity interpreter classified correctly 33 out of 34 substances for which two consensus ratings agree, toluene-2, 4 dilsocyanate being under estimated as not hazardous. Eleven other materials were considered, for which either the two consensus ratings disagreed or there was only one. Ten of these were classified "correctly".

We have applied these interpreters to the compounds whose CHETAH hazard ratings were developed in the first and second NBS Appraisals $[1 ; 8]$. The results are listed in Table A-1. The four CHETAH parameters are shown (by classes) first and then the impact and self reactivity interpreter results. Compounds in the table that were used by Treweek et al to develop the interpreters are marked by an asterisk. Using the data referenced and conventions given in the notes to the table we obtained the same classifications. Compounds are listed by sensitive functional groups, with those substances believed to be non-hazardous last.

If $C_{1}$ and $C_{2}$ compounds are ignored, the performance of the interpreters is very good. Nitriles appear to be overclassified by the impact interpreter. The CN group may behave as an (inert) pseudohalogen instead of an energy-rich grouping. Polyalcohols and sugars are the only classes overclassified. This can be traced to their low oxygen balance. It is easy to exclude these classes.

A different estimation method, "the L-System" has been developed by Dehaven [25b]. It combines thermodynamic and rate data to calculate the temperature at which $\mathrm{dT} / \mathrm{dt}$ is a maximum. There is a clear relationship between the derivation of the method and simple explosion theory. DeHaven tested the system against the list of 80 compounds used by 
Stull [6] in developing his "Reaction Hazard Index", compared the results against the NFPA ratings for these compounds, and developed a correlation between the L-system and NFPA. Agreement between the systems to \pm 1 (on the NFPA $0-4$ scale) is considered good performance. On this basis, the prediction was poor for only 3 compounds. This is appreciably better performance than for the Reactive Hazard Index when it is scaled, separately, to give the best fit to NFPA ratings. A comparison of this sort on this compounds has not yet been made for the CHETAH interpreter. 
Table A-1

CHETAH Parameters and Classification of Compounds by Interpreters

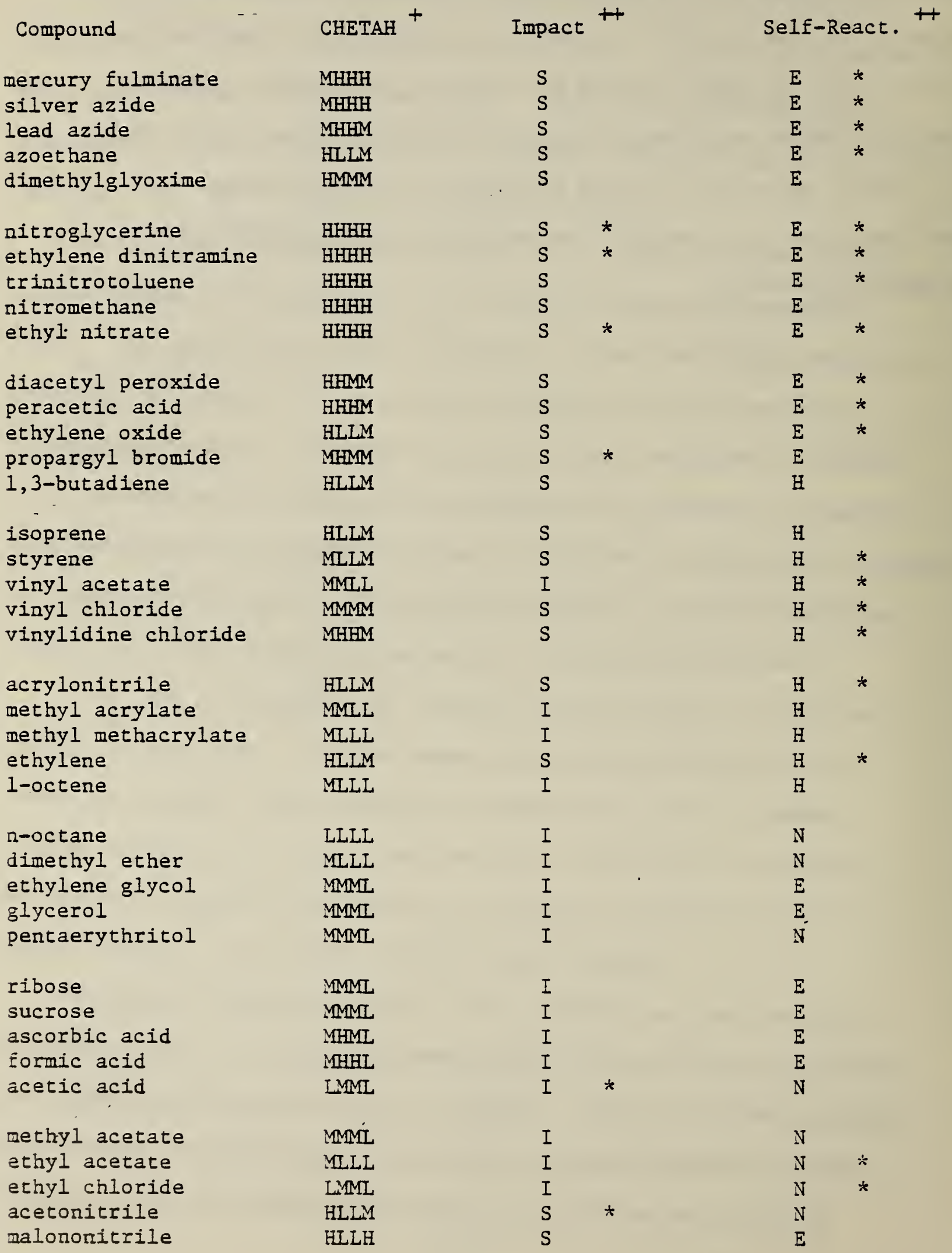


Compound

$$
\begin{aligned}
& \text { adiponitrile } \\
& \text { benzonitrile } \\
& \text { pyridine } \\
& \text { pyrimidine } \\
& \text { formamide }
\end{aligned}
$$

acetamide

$\mathrm{N}, \mathrm{N}$-dimethylformamide

urea

glycine

$\varepsilon$-caprolactain

triethylene diamine

Table A-1 (Continued)

$\operatorname{CHETAH}^{(+)} \quad \operatorname{Impact}^{(+)} \quad$ Self-React. ${ }^{(+)}$

HLLM

HLLM

HLLM

HLLM

MEMIL

LMM

MLLL

LHHL.

MHMI

MLLL

MLLL

$\begin{array}{ll}S & N\end{array}$ *

I N

I N

I E

I E

I N

I
$\dot{\mathbf{N}}$

the four CHETAH parameters are given in the order maximum enthalpy of decomposition, enthalpy of combustion minus maximum enthalpy of decomposition, oxygen balance and " $Y$ " (scaled square of the first parameter). $H=$ high, $M=$ Medium and $L=$ Low Hazard.

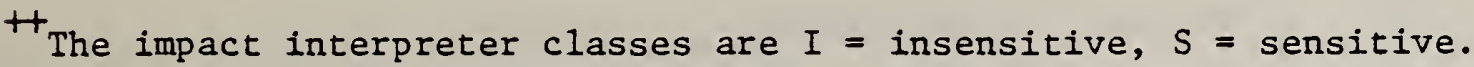
The self reactivity interpreter classes are $\mathrm{N}=$ non hazardous, $\mathrm{H}$ = hazardous or polymerizable, $E$ = Explosive.

\section{*}

*Used in constructing this interpreter. Numerical values for CHETAH parameters were taken from ref. [1] Tables $1-5$ and ref. [8], Tables 2-13, 18-22. Oxygen balances for the following compounds and incorrect in refs. $[1,8]$ and were recalculated (ref. 1, pg. 13): nitromethane, vinyl chloride, acetic acid, vinylidene chloride, vinyl acetate, methyl methacrylate and methyl acrylate. Bond dissociation energies for saturated analogs of olefinic monomers were provided by $W$. Tsang (personal communication). Those. for $\mathrm{C}-\mathrm{H}$ on the more highly substituted carbon were used. The enthalpy of polymerization of $\mathrm{C}_{8} \mathrm{H}_{16}(\mathrm{~g})$ was estimated as $-20 \mathrm{kcal}_{\mathrm{mol}}{ }^{-1}$. In the self-reactivity interpreter $-\Delta H$ (poly) per mole was used. 


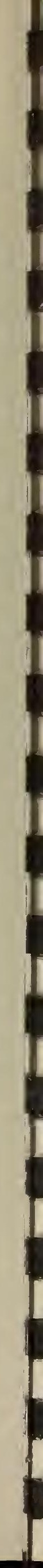


B. Thermal Explosion Study Notes

\section{B.1. Description of the Thermal Explosion Process}

The temperature-time sequence of events occuring when identical samples (i.e. composition, amount) of a thermally hazardous material at some low initial temperature, $T_{i n}$, are suddenly exposed progressively higher environmental temperatures, $T_{0}$, has been described in the second NBS hazards assessment (see [8], p. 122-123). The description is repeated here with some additional comments for sake of completeness.

In each experiment, the sample is placed in a container constructed from a metal having a large thermal conductivity. A thermocouple is imbedded in the material to monitor the temperature of the sample. After the sample, container, and thermocouple have come into thermal equilibrium and have an initial temperature $\mathrm{T}_{\text {in }}$, this assembly is suddenly immersed in a constant temperature bath held at some constant temperature $T_{0}$ greater than $\mathrm{T}_{\text {in }}$. Experiments differ in that $\mathrm{T}_{0}$ is held at progressively larger values. It is found that, if $T_{0}$ is less than some critical value, $T_{\star}$, the temperature, $\mathrm{T}$, of the material first increases rapidly from $\mathrm{T}_{\text {in }}$ to $T_{0}$. Then $T$ slowly rises slightly above $T_{0}$ due to self-heating to a maximum value that is still near $T_{0}$. Finally, $T$ decays back to $T_{0}$ after the decomposition of the material is complete.

If an experiment is now carried out with $T_{0}$ slightly higher than this critical bath temperature, a thermal explosion occurs. After $T$ has increased slowly to a value slightly higher than $T_{0}$, $T$ now abruptily (i.e. relative, to the time required to slightly exceed $\mathrm{T}_{0}$ ) starts to increase rapidly. This signals the onset of the thermal explosion. T rises to a very high maximum value (assuming the sample container and thermocouple 
are not destroyed) and again decays back to $T_{0}$ after the decomposition of the material is complete. Both the magnitude of the rapid rate of temperature rise and the maximum temperature of the sample in the thermal explosion increase as the total heat liberated per unit volume of the material, Q, by the decomposition reaction increases. However, the fraction of original material that decomposes prior to the explosion onset decreases as $Q$ increases. The fraction of material decomposed is of the order of $5 \%$ or less for explosives but may be $20 \%$ or greater for cellulosic materials. Notable exceptions are those materials which, while liberating large amounts of heat, decompose by an autocatalytic mechanism. In this case the fraction of material decomposed prior to the explosion onset may be as large or larger than those for cellulosic materials.

The $T$ versus time, $t$, curves for materials having small or large values of $Q$ are similar when $T_{0}$ is near but greater than its critical value. That is, in contrast to the non-explosive situation, the curvature of $T$ changes sign twice (negative to positive, then positive to negative) rather than always remaining negative. However, the curves differ in that for materials having a small $Q$, the time for the explosion onset to occur becomes less distinct (i.e. less easily identified) and the maximum temperature rise above $T_{0}$ is less.

Experimentally, it is found that when $T_{0}$ is very near the critical value, $T_{*}$, it is difficult to reproduce in separate experiments either the length of time it takes to explode (which increases very rapidly, the nearer $T_{0}$ is to its critical value) or, in fact, the actual occurrence of the explosion itself. This phenomenon, which is called part of the critical phenomenon, is due to the fact that only a slight perturbation in 
initial conditions (i.e. amount of sample, heat transfer to the container, etc.) or the bath temperature $T_{0}$ can shift the material from a explosive to a non-explosive condition or vice versa. The other part of the critical phenomenon is the seemingly abrupt and very rapid rise of the temperature to a very large maximum value (see [12], Chapter 6).

If experiments are made with a test tube of larger diameter, and, hence, a large amount of material, the critical value of $T_{0}$ is lowered. Hence, this critical ambient temperature is not a property of the material. If experiments are carried out in which a number of thermocouples are imbedded in the material, so that one can also monitor the temperature distribution of the material as a function of time, one finds that for non-explosive conditions the maximum temperature of the sample occurs in the center of the material. The temperature of the material decreases as one progresses from the center to the test tube wall (at $T_{0}$ ) roughly as an upside down parabola. For values of $T_{0}$ slightly above the critical value it is found that the rapid rate of temperature rise first occurs in the center of the sample. That is, the explosion is initiated at the center of the material. As $T_{0}$ is progressively raised to values much higher than the critical value of $T_{0}$, the explosion is initiated at positions in the material progressively closer to the walls of the test tube - ultimately at the material-test tube interface. This phenomena is called a progressive transition to the ignition regime. In the ignition regime, the surface of the material reaches an explosive condition whlle the temperature of the material at the center of the test tube has risen only slightly above $T_{\text {in }}$ 


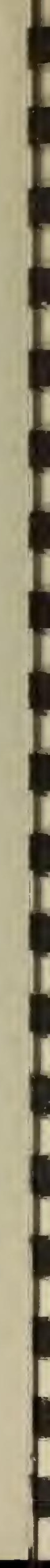




\section{B.2. Description of the Isothermal Model}

The simplest model used to describe thermal explosion phenomena is one in which the temperature distribution in the sample is ignored and the temperature of the sample is described by some average value $T$. While this model cannot explain "fine grained" effects such as the transition to ignition, it is regularly used by most investigators in the field of thermal explosions (theory or applications) either to explain the results of more realistic models qualitatively or as an aid in analyzing both the latter and experimental results. The model will apply to some laboratory or industrial stiuations quite closely (i.e. a well-stirred liquid or gas, see [13], p. 18). The five assumptions of the model are as follows.

1. The temperature of the material, $\mathrm{T}$, is uniform.

2. The reaction takes place ion one stage (i.e. simple kinetics) and is irreversible (i.e. far removed from equilibrium).

These two assumptions mean that the fraction of the original material reacted, $\eta$, at any time $t$ is given by:

$$
\frac{d \eta}{d t}=f(\eta) k_{T}
$$

where $f(\eta)$ is some arbitrary function of $\eta$ and $k_{T}$ is the specific rate. constant and is a function of $T$ alone. We shall assume ${ }^{+} k_{T}$ is expressed in the Arrhenius form:

$$
k_{T}=\operatorname{Aexp}(-E / R T)
$$

In eq. B-2, $A$ is the preexponential factor, $E$ is the activation energy, and $R$ is the gas constant. $A$ and $E$ are assumed to be independent of temperature.

\footnotetext{
The temperature dependence of $k_{T}$ need not be of the Arrhenius form in order for critical conditions tô exist.
} 
3. No phase transition occurs prior to explosion (1.e. melting, as usually occurs in explostves).

4. The container of the material remains closed and the rate of heat transfer, between the sample and container depends only on Newton's Law.

These two assumptions give for the heat balance equation for the entire material:

$$
C \frac{d T}{d t}=Q^{\prime} \frac{d \eta}{d t}-h^{\prime}\left(T-T_{0}\right)
$$

where $C$ is the total heat capacity, $Q^{\prime}$ is the total heat liberated by the reaction, and $h^{\prime}$ is an empirical average heat transfer coefficient between the sample and its container.

5. The characteristics of the reaction (A, E, $Q^{\prime}$ ) and the physical properties of the sample $\left(C, h^{\prime}\right)$ are independent of temperature and time over the ranges of interest. (This would exclude diffusion limited kinetics in eq. B-1 but does not require homogenous kinetics, per se.)

The last assumption is often justified by saying one is neglecting the temperature dependence of $\mathrm{C}, \mathrm{h}^{\prime}$, and $Q^{\prime}$ in comparison to $\mathrm{k}_{\mathrm{T}}$ which seems reasonable. In practice, this is justified provided one restricts the analysis both to a range of value of $T$ close to $T_{0}$ and also to the initial stages of those decomposition reaction in which gases are evolved (otherwise.one would not be able to assume $h^{\prime}$ is constant over even a short time or temperature interval).

The main problem in solving eqs. $B-1$ to $B-3$ as well as the corresponding equations for more detailed or realistic models of a self-reacting system is that their general solution must be obtained by numerical 
integration. To carry out these integrations efficiently, various dimensionless variables have been introduced. Becoming familiar with at least some of these variables is important not only to understand the physical meaning of the results of the numerical computations but also to cull out the useful practical results of approximate analytical solutions of eqs. B-1 to B-3. The variables are commonly used in almost all papers concerned with thermal explosion theory or its applications. A selected set of these variables is used here.

The analysis of eqs. B-1 to $B-3$, as well as those of more complicated models, may be grouped roughly into two categories: steady state theory and non stationary theory.

In steady state theory, the following hypothetical situation is posed. Suppose $f(n)$ is a constant, usually its maximum value, which we will call $f\left(n_{m}\right)$ (i.e. the reaction mechanism is zero order kinetics). The questions asked are: a) At what values of $T$ are the rate of heat generation, $\dot{q}_{1}$, equal to the rate of heat loss, $\dot{q}_{2}$ ?, and $b$ ) That is the stability of these equilibrium states? In the model cited above:

$$
\begin{aligned}
& \dot{\mathrm{q}}_{1}=\mathrm{Q}^{\prime} \mathrm{f}\left(\eta_{\mathrm{m}}\right) \operatorname{Aexp}(-E / R T) \\
& \dot{q}_{2}=\mathrm{h}^{\prime}\left(\mathrm{T}-\mathrm{T}_{0}\right)
\end{aligned}
$$

By stability is meant the following. Suppose $T$ is displaced by an infinitesimal amount from its equilibrium value. If $T$ always returns to its equilibrium value whether the displacement is positive or negative, the state is sald to be stable or subcritical. If $T$ returns to its equilibrium value when $T$ is displaced in one direction but not the other, the state is called metastable (as at the critical value of $T_{0}$ in the previous section -this is called the critical state). The state is said 
to be unstable if the initial values of $Q^{\prime}, f\left(n_{m}\right), A, E$, and $h^{\prime}$ (and the value of $T_{0}$ ) are such that $\dot{q}_{1}$ can never equal $\dot{q}_{2}$.

In non-stationary analyses, the more complicated problem of understanding the temperature-time solutions of eqs. B-1 to $B-3$ is investigated in terms of the results of the previous steady state analysis. Non-stationary analyses can be classified into two groups. The first is the simpler case where reactant consumption is ignored (as in the steady state analysis). This applies to a first approximation to the events occuring prior to the onset of an explosion in materials having a large heat of decomposition per unit volume of the material. The second group is the more difficult case where the effect of reactant consumption or material decomposition on $f(n)$ prior to the onset of explosion cannot be ignored. This is the case of interest to DOT.

Because it is impossible to understand the results of analyses in this last category without first understanding the results of the other simpler steady state analysis and non stationary analysis neglecting reactant consumption, a brief summary of each type of analysis is given below in sections B.3. and B.4. using the simple model already described above. 


\section{B.3. Steady State Analysis of the Isothermal Model}

This analysis was originally developed by Semenov (see [29]). Plots of $\dot{q}_{1}$ in eq $B-4$ for a given material and various values of $\dot{q}_{2}$ given by eq. B-5 versus $T$ are usually referred to a Semenov diagram whatever the temperature dependence of $\dot{q}_{1}$ and $\dot{q}_{2}$. A Semenov diagram for values of $T$ near $T_{0}$ (the only case of interest) is given in the figure on page 125 of ref [8] in which $\dot{q}_{2}$ is plotted for various values of $T_{0}$ in analogy to the results of the explosion temperature experiment described in section B.I. and in the second NBS hazard assessment. It is simpler to analyze a Semenov diagram by considering $T_{0}$ to be constant and to examine the situation as $\dot{q}_{2}$ varies due to changes in $h^{\prime}$. A diagram of this situation is sketched in figure $B-1$ for a material for which $Q$ ' is positive. One can see that for a large value of $h^{\prime}$, case (1), $\dot{q}_{1}$ and $\dot{q}_{2}$ intersect at only one temperature $\left(T_{\ell}\right)_{1}$, and the state is stable. In case $(2), h^{\prime}$ is less than in case (1). $\dot{q}_{1}$ and $\dot{q}_{2}$ intersect at two temperatures ${ }^{+}$, and only the state at temperature $\left(\mathrm{T}_{\ell}\right)_{2}$ is stable. Assuming the material is originally at some temperature, $T_{i n}$, less than or equal to $T_{0}$, $T$ increases only to $\left(\mathrm{T}_{\ell}\right)_{2}$. As $\mathrm{h}^{\prime}$ is decreased to case (3), $\mathrm{T}_{\ell}$ and $\mathrm{T}_{\mathrm{u}}$ approach $\mathrm{T}_{c}$ and at this point both $\dot{\mathrm{q}}_{1}=\dot{\mathrm{q}}_{2}$ and $\mathrm{d} \dot{\mathrm{q}}_{1} / \mathrm{dT}=\mathrm{d} \dot{\mathrm{q}}_{2} / \mathrm{dT}$. One can see this state is metastable ( $i . e$. increase $T$ slightly) and corresponds to the critic̈al condition. Smaller values of $h^{\prime}$ will result in a thermal explosion. Solving eqs. B-4 and B-5 for the critical condition, one obtains, using simple algebra and assuming $4 \mathrm{RT} / \mathrm{E}$ is less than one (which applies to the cases of interest):

\footnotetext{
$\bar{t}_{\text {Because } \exp }(-E / R T)$ versus $T$ flattens out at high temperatures, there will actually be three temperatures at which $\dot{q}_{2}$ intersects $\dot{q}_{1}$ in case (2), and in case (3) $\dot{q}_{2}$ will also intersect $\dot{q}_{1}$ at a very high temperature. For values of $h^{\prime}$ near the critical value, case (2), $\mathrm{T}_{1}$ and $\mathrm{T}_{\mathrm{u}}$ are near $\mathrm{T}_{\mathrm{C}}$. The other intersection temperature is very large and is approximately equal to the higher intersection temperature for case ( 3 ) which is $\mathrm{T}_{0}+$ $\mathrm{T}_{\mathrm{O}}\left(E / R \mathrm{R}_{0}\right)^{2}$. The latter value is far outside the validity of equations $\mathrm{B}-1$ to $\mathrm{B}-3$.
} 


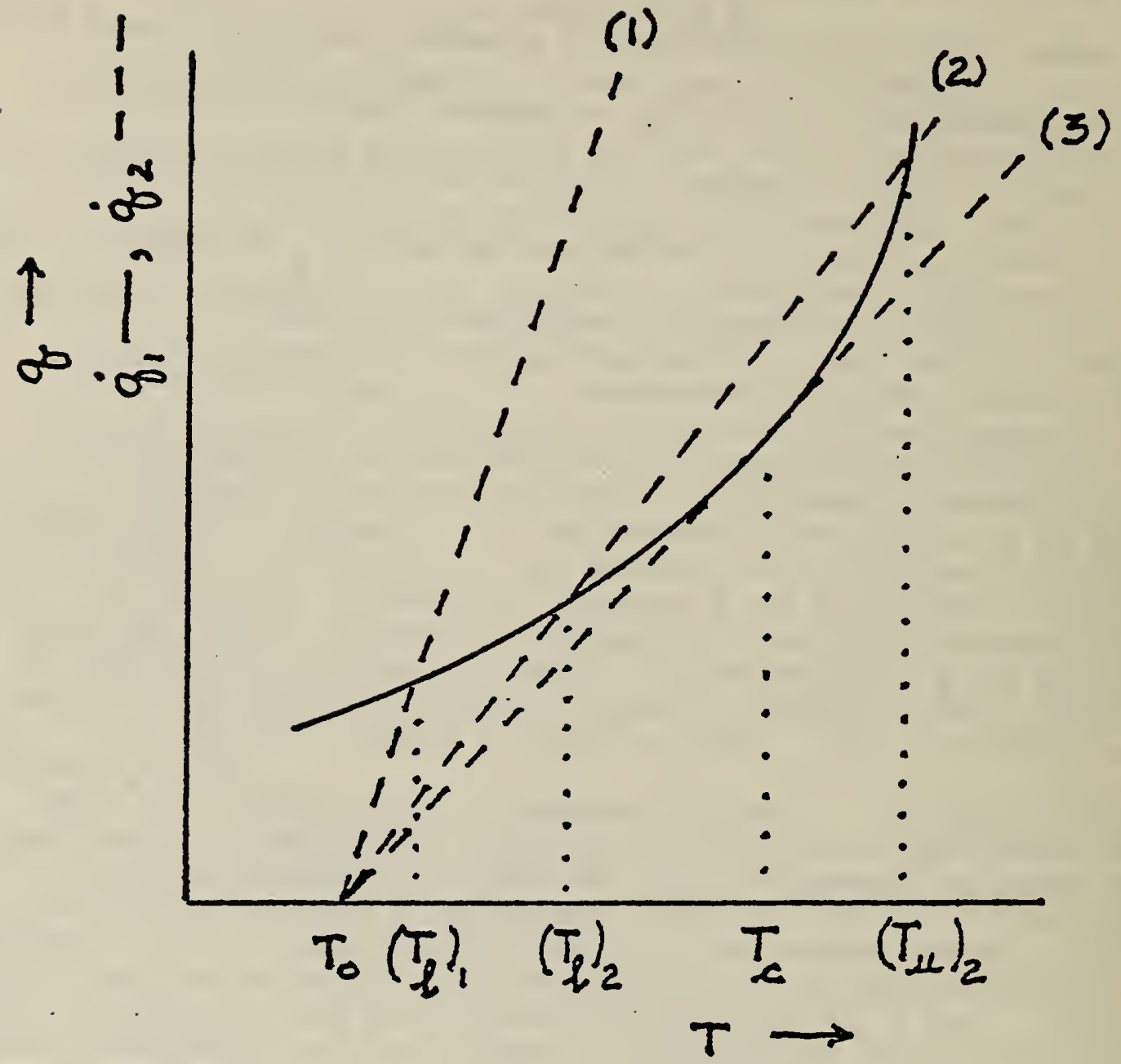

Figure B-1. Semenov Diagram

$\dot{q}_{1}$, - rate of generation of heat by sample; $\dot{q}_{2},--$, rate of heat loss of sample; $T$, temperature of sample, $\mathrm{T}_{\mathrm{O}}$, temperature of sample environment; $\left(\mathrm{T}_{\ell}\right)_{1}, \mathrm{~T}$ at intersection $\dot{q}_{1}$ and $\dot{q}_{2}$, case $(1) ;\left(\mathrm{T}_{\ell}\right)_{2},\left(\mathrm{~T}_{\mathrm{U}}\right)_{2}, \mathrm{~T}$ at intersection of $\dot{q}_{1}$ and $\dot{q}_{2}$, case (2); $T_{c}, T$ at tangency of $\dot{q}_{1}$ and $\dot{q}_{2}$,
case (3). 


$$
T_{c}=T_{\star}+T_{*}\left[R T_{*} / E \ldots\right]
$$

and

$$
\delta^{\prime}=\delta_{c}^{\prime}
$$

where

$$
\delta_{c}^{\prime}=\frac{1}{e}\left[1+R T_{o} / E \ldots\right] ; e=2.718 \ldots
$$

and

$$
\delta^{\prime}=\frac{Q^{\prime}}{h^{\prime}} \operatorname{Af}(n) \frac{E}{m_{R_{0}}{ }^{2}} \exp \left(-E / R T_{0}\right)
$$

$T_{*}$ is the value of $T_{0}$ computed from eq. $B-7$ using eq. $B-8$ and $B-9$. Values of $\delta^{\prime}$ greater than $\delta_{c}^{\prime}$ will result in $\dot{q}_{1}$ always being greater than $\dot{q}_{2}$ so that a thermal explosion occurs. $\delta$ is a dimensionless variable that is analogous to that Introduced by Frank-Kamenetski1 (see [12], Chapter 7) in connection with a more complex model than that being discussed here. The value of $T_{c}$ when $\delta^{\prime}=\delta_{c}^{\prime}$, is very close to $T_{*}$ since $E$ is much larger than $\mathrm{RT}_{0}$ (1.e. If $\mathrm{E}=35 \mathrm{kcal} / \mathrm{mol}$ and $\mathrm{T}_{0}$ is $100^{\circ} \mathrm{C}, \mathrm{T}-\mathrm{T}_{*}$ is 8 $\left.{ }^{\circ} \mathrm{C}\right)$.

The physical interpretation of the parameter $\delta^{\prime}$ in eq. B-9 is that it is the ratio of $\mathrm{d} \dot{q}_{1} / d T$ divided by $\mathrm{d} \dot{q}_{2} / d T$, both being evaluated at the ambient temperature, $T_{0^{\circ}}$ Both of these derivatives must be positive (i.e. materials that decompose with absorption of heat are not hazardous) and $\mathrm{d} \dot{\mathrm{q}}_{1} / \mathrm{dT}$ must increase more rapidly with increasing $\mathrm{T}$ than $\mathrm{d} \dot{\mathrm{q}}_{2} / \mathrm{dT}$ in order for critical conditions to exist. It is clear that the latter requirement was automatically satisfied by assuming $k_{T}$ can be expressed in the Arrhenius form with both $A$ and $E$ having positive values and by assuming $\dot{q}_{2}$ increases linearly with $\mathrm{T}$, the simplest case. 
Critical conditions can also arise naturally whenever $k_{T}$ mimics the Arrhenius form over a limited temperature range. (The dependence of $\dot{q}_{1}$ and $\dot{q}_{2}$ at values of $\mathrm{T}$ much higher than $\mathrm{T}_{0}$ will, however, determine the maximum possible value of $T$ that can be attained if $\delta^{\prime}$ exceeds $\delta_{c}^{\prime}$ ) To illustrate, it is first useful to digress and introduce the dimensionless temperature $\theta$ by expressing $k_{T}$ in terms of $\mathrm{T}-\mathrm{T}_{0}$ as follows:

$$
k_{T}=k_{T_{0}} \exp [\theta /(1+\beta \theta)]
$$

where

$$
\begin{gathered}
\mathrm{k}_{\mathrm{T}_{0}}=\operatorname{Aexp}\left(-\mathrm{E} / \mathrm{RT} \mathrm{T}_{0}\right)=1 / \tau_{\mathrm{r}} \\
\theta=\mathrm{E}\left(\mathrm{T}-\mathrm{T}_{0}\right) / \mathrm{RT}_{\mathrm{O}}^{2} \\
\beta=\mathrm{RT}_{\mathrm{o}} / \mathrm{E}
\end{gathered}
$$

$k_{T_{0}}$ is the rate constant when the temperature of the material equals $T_{0}$ Since $k_{T_{0}}$ has the dimensions of reciprocal time, it is often expressed as the reciprocal of a reaction time for which we use the symbol $\tau_{r^{*}}$ (It is evident that since $\theta$ is $\left(\mathrm{T}-\mathrm{T}_{0}\right) / \mathrm{T}_{0} \beta$ where $\beta$ is quite small, $\theta$ will be small only if $\left(\mathrm{T}-\mathrm{T}_{\mathrm{o}}\right) / \mathrm{T}_{\mathrm{o}}$ is sma11.) Using eq. $\mathrm{B}-10$, one can write the rate and heat balance equations in terms of the dimensionless temperature, $\theta$, as

$$
\begin{array}{rlc}
\frac{d \eta}{d t}=k_{T_{0}} f(\eta) \exp [\theta /(1+\beta \theta)] & B-14 \\
\frac{d \theta}{d t} & =B \frac{d \eta}{d t}-\frac{\theta}{\tau_{q}} & B-15 \\
& =B k_{T_{0}}\left[f(n) \exp [\theta /(1+\beta \theta)]-\frac{\theta}{\delta^{\prime}} f\left(n_{m}\right)\right] B-15 a
\end{array}
$$

where

and

$$
B=\frac{E}{R T_{0}^{2}} \frac{Q^{\prime}}{C}=\frac{1}{\gamma}
$$

$$
\tau_{q}=\mathrm{c} / \mathrm{h}^{\prime}
$$


One can see from eq. $B-15$ that $B$ or $1 / \gamma$ (another symbol often used) is the maximum rise in $\theta$ above $\theta=0, \Delta \theta$, that can be produced by decomposition (i.e. adiabatic conditions). In this case, the thermal relaxation time, $\tau_{q}$, is set equal to infinity and eq. B-15 yields $\Delta \theta=$ B since $\Delta \eta=$ 1.

Returning to the question of what the temperature dependence of $k_{T}$ must be to have critical conditions that are the same as when $k_{T}$ has the Arrhenius form, consider first the case where the argument of the exponential in eq. B-10 is replaced $\theta$, the so called exponential approximation. $\exp (\theta)$ differs from $\exp (\psi)$, where $\psi$ is $\theta /(I+\beta \theta)$, by at most $20 \%$ in the range of $\theta$ from -2 to +2 when $\beta=0.05$. The value of $\delta_{c}^{\prime}$ for the exponential approximation is the same as that given in $B-9$ except that $B=$ $\mathrm{RT}_{\mathrm{o}} / \mathrm{E}$ must be set equal to 0 and $\theta_{c}=1$ in Eq. B-6. Alternatively, suppose one replaces $\exp (\psi)$ with $1+a_{1} \theta+a_{2} \theta^{2}$ where $a_{1}=e-2$ and $a_{2}=1$, the so called quadratic approximation (see [13] p. 27). This mimics exp ( $\psi$ ) over the range of $\theta$ from 0 to 2 but is less than $\exp (\psi)$ by $10.5 \%$ at $\theta=+2$ (if $\beta=.05)$. The critical conditions are the same as for the exponential approximation. In both of these examples, $\delta$ ' is given by

$$
\delta^{\prime}=B k_{T_{0}} f(n){ }_{m} \tau_{q}=B f\left(n_{m}\right) \tau_{q} / \tau_{r}
$$

which is the form of eq. B-9 in terms of the new variables.

As to the question of what form $k_{T}$ must have in order for a thermal - explosion to occur (if conditions are correct), one can see that the critical phenomenon does not naturally arise if $\exp (\psi)$ is replaced by $a_{1}+$ $a_{2} \theta$ where $a_{1}$ and $a_{2}$ are arbitrary but positive. Thus, we can infer that, qualitatively, the conditions for a substance to be potentially thermally unstable are that $Q^{\prime}$ be positive and that $\delta^{\prime}$, which can be written in 
in the form of eq. B-19, increase very. rapidly with $\mathrm{T}_{a}$ for an appreciable range of temperatures.

$$
\delta^{\prime}=\left[\mathrm{d} \dot{q}_{1} / \mathrm{dT}\right]_{\mathrm{T}_{0}} /\left[\mathrm{d} \dot{\mathrm{q}}_{2} / \mathrm{dT}\right]_{\mathrm{T}_{0}}
$$

For example, critical conditions will be exhibited by heterogeneous reactions which are diffusion limited provided this limitation applies only to the higher portion of ambient temperature regime of interest. An example of a Semenov diagram analysis of this type of mechanism is given by Frank-Kamenetskii (see [12], Chapter 9) for the case where

$$
\frac{d \eta}{d t}=\frac{\phi k_{T}(1-\eta)}{k_{T}+\phi}
$$

where $k_{T}$ is of the Arrhenius form and $\phi$ is a constant (i.e. $\phi$ is approximately independent of temperature). At small values of $\mathrm{T}, \mathrm{d} n / \mathrm{dt}$ depends on $T$ as $k_{T}$ while at high values of $T$, dn/dt is independent of $T$. A short physical description of this type of problem in context of an oxygen combustion reaction is given by Thomas (see [15] p. 68).

The effect of the mechanism of the reaction, reactant consumption, and previous thermal history of the material on the critical conditions is contained in the quantity $f\left(\eta_{m}\right)$ of eq. B-9. If $f(n)$ is simple

$$
f(n)=(1-n)^{n}
$$

where $\mathrm{n}$ is greater than zero, one can see $f\left(\eta_{m}\right)=f(0)=1$. Thus, qualitatively, the effect of reactant consumption for simple mechanism will be to increase the value of $T_{0}$ in eq. $B-9$ and, hence, $T_{c}$ in eq. $B-6$ at the critical condition. In other words, if a real material is in a supercritical state (one which results in a thermal explosion), the temperature always slowly rises to some value above $T_{*}$ given by eq. B-6 when $f(n)$ is constant before an explosion occurs. An alternative way of obtaining this 
result is to note that $\dot{q}_{1}$ is lowered in the Semenov diagram so that the point of tangency of $\dot{q}_{1}$ and $\dot{q}_{2}$ is shifted to a higher value of $T$ for $n>0$ as compared to $\eta=0$. Clearly, a partially decomposed sample that self-reacts according to the simple scheme of eq. B-21 will be less dangerous than a newly prepared sample of the material.

Unfortunately all materials do not decompose by this simple kinetic scheme, although portions of a $d n / d t$ versus $n$ plot may show this behaviour. A very great number of materials decompose by an autocalytic mechanism that may be nominally represented in its simplest form by the equation

$$
f(n)=\left(n+n_{0}\right)(1-n) ; n_{0} \ll 1
$$

In this case $f\left(n_{m}\right)$ occurs at $n_{m}=\left(1-n_{0}\right) / 2$ (if $n_{0}=0$, this a first order autocalytic reaction) and $f\left(n_{m}\right)=\left(1+n_{0}^{2}\right) / 4$. Clearly an older sample of a material decomposing by eq. B-22 will be more dangerous than a freshly prepared sample (when $n=0, f(0)=n_{0} ; n_{0}<\left(1-n_{0}\right)^{2} / 4$ if $\eta_{0}<1$ ). This is usually the case for many of the known explosive materials.

It is important to note that the initial stages of the decomposition of many materials may involve two or more exothermic reactions with which are associated different values of $Q^{\prime}, A$, and $E$. Analysis of the critical conditions proceeds in essentially the manner as described above but are considerably more complex, as even the simple problem studied by Bowes" [31] illustrates.

As it stands, eq. B-9 or B-19 is not as informative as one might like because $h^{\prime}$ is an empirically determined heat transfer coefficient and the dependence on sample size, geometry, and thermal conductivity is hidden in the quantity in the expression for $Q^{\prime} / h^{\prime}$. To indicate how this dependence may be qualitatively built into eq. B-9, one may proceed as follows. 
Suppose the material is a continuous liquid or a solid "chunk" of material having a thermal conductivity $\lambda$ and a symetrical shape similar to its container with a characteristic dimension $r$ (i.e. for a sphere, $r$ would be the radius). Then for this solid one may write

$$
\frac{1}{h}=\frac{1}{h}+\frac{1}{h_{0}}
$$

where $h$ is the heat tranfer coefficient of the material if its outer surface is in perfect thermal contact with its container and $h_{0}$ is the heat transfer coefficient of the material and container in the limit when the temperature of the material is always uniform. $h$ and $h_{0}$ can be expressed as the product of the surface area of the material, $S$, and heat transfer coefficients per unit area, $\alpha$ and $\alpha_{0}$ :

$$
\mathrm{h}=\alpha \mathrm{S} ; \mathrm{h}_{0}=\alpha_{0} \mathrm{~s}
$$

Provided heat transfer in the material occurs solely by conduction, one can express $\alpha$ approximately in the form

$$
\alpha=\lambda g_{1} / r
$$

where $g_{1}$ is a function of geometry alone provided $\alpha, \alpha_{0}$, and $S$ are independent of temperature and time. Equation B-25 accounts for the temperature gradients in the material due to heat transfer in the absence of self heating. Expressing $Q^{\prime}$ as the product of the heat liberated per unit. volume of the material, $Q$, and its volume, $V$, we have for $Q ' / h^{\prime}$ :

$$
\frac{Q^{\prime}}{h^{\prime}}=Q V\left(\frac{1}{h}+\frac{1}{h_{0}}\right)=\frac{Q V r}{s \lambda g_{1}}\left(\frac{\lambda g_{1}}{r \alpha_{0}}+1\right)
$$

Equation B-26 requires that $Q$ be the same at every point of the material (or that $Q$ be interpreted as the volume average of $Q^{\prime}$ ) and that $g_{1}$ in eq. B-25 be approximately the same when self-heating occurs. V/S can be written in the form 


$$
\frac{\mathrm{V}}{\mathrm{s}}=\mathrm{rg}_{2}
$$

where $g_{2}$ is a function of geometry alone (but different from $g_{1}$ ) provided $Q$ and $V$ are also independent of temperature near $T_{0}$. The quantity $\alpha_{0} r / \lambda$ is called the Biot number, $B 1$, which characterizes the relative effect of the internal heat transfer in the material and heat transfer between the material and its environment. (As Bi get large, the sample approaches perfect thermal contact with its container.) Thus, one may write

$$
\frac{Q^{\prime}}{h^{\prime}}=\frac{Q r^{2}}{\lambda} \frac{g_{2}}{g_{1}}\left(\frac{g_{1}}{B i}+1\right)
$$

This permits us to write eqs. $B-7, B-8$, and $B-9$ in the form

$$
\delta=\delta_{C}
$$

where

$$
\delta_{c}=\frac{1}{e} \frac{g_{1}}{g_{2}}\left(1+\frac{R T o}{E} \ldots\right)\left(\frac{B i}{B i+g_{1}}\right)
$$

and

$$
\delta=\frac{Q r^{2} A}{\lambda} f\left(\eta_{m}\right) \frac{E}{R_{0}^{2}} \exp \left(-\frac{E}{R T_{0}}\right)
$$

The form of eqs. B-29 to B-31 makes $\delta$ of eq. B-31 identical to the critical parameter for the one dimensional models that take into account temperature gradients in which heat transfer takes place only by conduction (see section B.6.). Comparing eq. B-30 with the results of these models at infinite Biot numbers yields values of $g_{1} / \mathrm{eg}_{2}$ of $0.88,2.00$, and 3.32 for the infinite slab (half thickness $r$ ), infinite cylinder (radius $r$ ) and sphere (radius $r$ ). From thermal relaxation time considerations ${ }^{+}$one' would predict that the corresponding values of $g_{1} / \mathrm{eg}_{2}$ are $0.91,2.13$, and 3.63 .

\footnotetext{
The thermal relaxation time, see eq. B-1, can be written in the form $\mathrm{r}^{2} \mathrm{~g}_{2} / \mathrm{g}_{1} \mathrm{~K}$ where $\mathrm{K}$ is the thermal diffusivity. Comparison with the longest thermal relaxation times of the corresponding geometries, when $\alpha_{0}$ is zero, gives the values of $g_{1} / \mathrm{eg}_{2}$ cited. This is the first approximate method discussed by Thomas [32].
} 
Since $g_{2}$ equals $1,1 / 2$, and $1 / 3$ for the infinite slab, infinite cylinder, and sphere, respectively, one can also obtain values of $g_{1}$ for each onedimensional geometry. Using the thermal relaxation values of $g_{1} / e g_{2}$ and $g_{1}$ values calculated as indicated, one finds that $\delta_{c}$ for the infinite slab and cylinder given by eq. B-30 agrees within $7 \%$ with the exact values (see [31]) for all Biot values greater than $10^{-4}$.

Other methods besides thermal relaxation times may be used to estimate $\delta_{c}$ for the geometries mentioned above as well as other geometries (see [13], section 3.3, and Appendix B.6).. While a detailed check of all known results has not been made, it seems probable that $\delta_{c}$ can be estimated by methods involving an extension of the isothermal model to about $10 \%$ or less ${ }^{+}$.

In order to draw qualitative conclusions, it is somewhat easier to use the critical parameter $\delta^{\prime}$ of eqs. B-8 and B-9 with eq. B-9 being replaced by eq. B-3la:

$$
\delta^{\prime}=\frac{Q r^{2}}{\lambda} \frac{g_{2}}{g_{1}}\left(\frac{g_{1}}{B i}+1\right) \operatorname{Af}\left(\eta_{m}\right) \frac{E}{R T_{0}^{2}} \exp \left(-\frac{E}{R T_{0}}\right)
$$

Equation B-3la indicates that, for a continuous material undergoing a single exothermic homogenous decomposition reaction, there is, for any given Biot number, a maximum critical size for each ambient temperature, assuming it is constant and uniform, that must not be exceeded if a thermal explosion is to be avoided. Using $\delta^{\prime}$ as an indicator of the instability of the material, one can say that this critical size is approached at a rate (i.e. by $\delta^{\prime}$ ) proportional to the first power of the diameter if $B i$

\footnotetext{
In the literature, the dependance of $\delta_{c}$ on $B i$ is often approximated as being independent of geometry. For the three symmetrical cases considered this dependance would be given by $g_{1}=e$ for eqs. $B-28$ and $B-30$. $\mathrm{BI} /(\mathrm{Bi}+\mathrm{e})$ usually appears as $\frac{\mathrm{BiZ}}{2} \exp [+(\mathrm{Z}-2) / \mathrm{Bi}]$ where $\mathrm{Z}$ is $\mathrm{Bi}^{2}+4-$ Bi. (see eq. 3 of ref. [14]). The latter matches $\delta_{c}$ to about $10 \%$.
} 
proportional to the first power of the diameter if $B i$ is small and the second power of the diameter if $\mathrm{Bi}$ is large in comparison to $\mathrm{g}_{1}$. The former case would apply to liquids or gases where convection (free or forced due to gases liberated in the decomposition) dominates heat transfer due to conduction. Small Biot numbers correspond to the physical situation where the temperature difference between the surface of the material and its container is much greater than the temperature gradient in the material ${ }^{+}$. Reducing the Biot number by reducing $r$ or by reducing $\alpha_{0}$ have opposite effects on the thermal stability; the latter increases the thermal instability of the material in the sense that it lowers the critical temperature of the material.

The preceding simple. extension of the simple model, which has been discussed by many authors (see [14] section 2), works primarily because prior to the onset of a thermal explosion, the temperature gradient in the material is small. The important step in the analysis is the expressIon in eq. B-25 which is a statement of the fact that rate of loss of heat of a symmetrically shaped chunk of material in perfect thermal contact with its container, $\dot{q}_{2}$, is given by

$$
\dot{q}_{2}=\oint_{S} \lambda \phi_{s}(\nabla T)_{s} d S
$$

where $(\nabla T)_{S}$ is the gradient of $T$ at and normal to the surface of the material. One obtains eq. B-25 by dividing by $S$ and replacing $(\nabla T)_{S}$ by

$$
(\nabla T)_{S}=g_{1}\left(T-T_{s}\right) / r \quad B-33
$$

where $T$ and $T_{S}$ are the average and surface temperatures, respectively of the material.

\footnotetext{
From eqs. $\mathrm{B}-32, \mathrm{~B}-33$, and $\mathrm{B}-24$ one has from steady state considerations that $\lambda g_{1}\left(\bar{T}-T_{S}\right) S / r \cong \alpha_{0} S\left(T_{S}-T_{0}\right)$ where $\bar{T}$ is the average temperature of the material and $T_{S}$ is the temperature of the material adjacent to the environment. Hence, $\left(\overline{\mathrm{T}}-\mathrm{T}_{\mathrm{S}}\right) /\left(\mathrm{T}_{\mathrm{S}}-\mathrm{T}_{0}\right)=\mathrm{Bi} / \mathrm{g}_{1}$.
} 
Neither of these relations will necessarily apply to a material made up of solid particles. Our literature survey has not turned up any detailed analysis of this case -one of practical importance. Inclusion of the effect of the sample container also has not been worked out in detail (though the success of the previous extension of the simple model suggests it can be treated in an analogous fashion ${ }^{+}$). All of the preceding comments indicate that theoretical solutions are still needed to provide a complete set of relations to predict the behaviour of materials in shipping containers from laboratory test measurements on small samples.

The result would be of the form $\delta_{c}=\frac{1}{e} \frac{g_{1}}{g_{2}}\left(1+\frac{R T_{o}}{E} \ldots\right) B i\left[B i\left(\frac{t_{c} \lambda g_{1}}{r \lambda_{c} g_{c}}+1\right)+g_{1}\left(\frac{\alpha_{o}}{\alpha_{e}}+1\right)\right]^{-1}$

where $t_{c}$ is the thickness of the container which has a thermal conductivity, $\lambda_{c}$ and $\mathrm{a}$ heat transfer coefficient per unit area, $\alpha_{e}$, to the external environment. $g_{c}$ is analogous to $g_{1}$ of eq. B-25 except that it applies to the container. Thus, if the container has a large thermal conductivity, small thickness, and a large heat transfer coefficient with respect to the outside environment so, that $\left(t_{e} \lambda g_{1}\right) /\left(r \lambda_{c} g_{c}\right) \ll 1$ and $g_{1} \alpha_{0} / \alpha_{e}<$ 1 the effect of the presence of the container on $\delta_{c}$ is negligible. In effect, the container becomes the environment of the material. 


\section{B. 4 Nonstationary Analysis of the Isothermal Model, $f(n)$ Constant}

An approximate idea of the relation between the time required for a thermal explosion to take place (when the material is in a supercritical state) and the parameters determined in the previous section can be obtained by neglecting the consumption of material due to decomposition on $f(n)$. As such, the comments apply only to real explosives and then only approximately. As in the previous section, one assumes that $f(n)$ in eq. B-14 is replaced by a constant value; we shall use $f\left(\eta_{m}\right)$. Rearranging and integrating eq. $B-15 a$, one can write for the time, $t^{\circ}$, to explosion that is. defined to occur at temperature $\mathrm{T}_{e}$ :

$$
t^{0}=\frac{1}{B k_{T_{0}} f\left(\eta_{m}\right)} \int_{\theta}^{\theta} e \frac{d \theta}{\operatorname{in}\left[\exp \left(\frac{\theta}{1+\beta \theta}\right)-\frac{\theta}{\delta^{1}}\right]}
$$

where

$$
\begin{aligned}
\theta_{e} & =\frac{E}{R T_{0}^{2}}\left(T_{e}-T_{0}\right) \\
\theta_{\text {in }} & =\frac{E}{R T_{0}^{2}}\left(T_{\text {in }}-T_{0}\right) \\
\delta^{\prime}>\frac{1}{e} & \text { or } \frac{1}{\delta}=e-\Delta ; e>\Delta>0
\end{aligned}
$$

(a supercritical state) B-36

$T_{\text {in }}$ is the initial temperature of the material at zero time when the material is abruptly exposed to some higher ambinet temperature $T_{0}$ that will cause the material to explode. $\theta_{\text {in }}$ is negative. The quantity $\left(B k_{T_{0}}\right)^{-1}$ is called the adiabatic induction time, $\tau_{\infty}$, for reasons given later. Thus

$$
\tau_{\infty}=\frac{1}{B k_{T_{0}}}=\frac{\tau_{r}}{B}=\gamma \tau_{r}
$$

In the following discussion we shall consider the variation of $t^{\circ}$ due to changes in $\tau_{\infty}$, the integral in eq. $B-34$, and $f\left(\eta_{m}\right)$ in that order. 
It is important to note that the value of the integral is related to $\tau_{\infty}$ because from eq. $B-18$ we have

$$
\delta^{\prime}=f\left(\eta_{m}\right) \tau_{q} / \tau_{\infty}
$$

and for explosive states $\delta^{\prime}$ must be obey eq. B-36. (thus, in supercritcal or explosive states, $\tau_{\infty} / f\left(\eta_{m}\right)$ must always be less than e times $\left.\tau_{q}{ }^{\cdot}\right)$ Thus, $t^{0}$ is a function of the four parameters $\tau_{\infty} / f\left(\eta_{m}\right)$ or $\tau_{q}$, the ratio $\tau_{\infty} / f\left(\eta_{\mathfrak{m}}\right) \tau_{q}, \theta_{i n}$, and $\theta_{e}$. It will be seen below that $t^{0}$ is relatively insensitive to the choice of $\theta_{e}$ so long as it is large in comparison to one. Thus, for fixed $\tau_{q}$ and $\theta_{\text {in }}$, the greater the supercriticality of the state (i.e. the greater $\delta^{\prime}$ above $1 / e$, the less $1 / \delta^{\prime}$ below $e$, or the greater $\Delta$ in eq. $B-36)$, the smaller $t^{\circ}$ becomes because both $\tau_{\infty}$ and the integral in eq. B-34 decrease. This situation would apply to the explanation for the decreasing time to explosion for progressively increasing ambient temperatures in the explosion temperature test in section B.1. Also, this suggests that activation energies based on time to explosion mesurements that assume

$$
\ln t^{0}=\ln \tau_{\infty}+a_{1}
$$

where $a_{1}$ is a constant independent of $T_{0}$ will be subject to some error. because $a_{1}$ changes with $T_{0}$ through $\delta^{\prime}$ in the integral of eq. B-34. This has been verified in detail by Zinn and Mader [33] for more complex models that include the effect of temperature gradients in the material.

In order to understand the effect of the integral in eq. $B-34$ on $t^{\circ}$ in more detail, consider, first, $t^{0}$ for explosive states near the critical state -when $\Delta$ is small in eq. B-36. From the section B.4. we know that the sample will explode near $\theta_{e}=1$ so that $\theta_{e}$ should be some value greater than one when $d \theta / d t$ is quite large. It is convenient under these cir- 
cumstances to break $t^{\circ}$ into the sum of the time, $t_{q}^{\circ}$ required to heat the sample from $T_{i n}$ to $T_{o}$ (i.e. $\theta_{i n}$ to 0 ) and from $T_{0}$ to $T_{e}$ (i.e. 0 to $\theta_{e}$ ):

$$
t^{0}=t_{q}^{0}+t_{i}^{0}
$$

where

$$
\begin{gathered}
t_{\mathrm{q}}^{0}=\frac{\tau_{\infty}}{\mathrm{f( \eta _{ \textrm {m } } )}} \int_{\theta}^{\theta^{i}} \frac{\mathrm{d} \theta^{\prime}}{\left[\exp \left[\frac{-\theta^{\prime}}{1-\beta \theta^{\prime}}\right]+\frac{\theta^{\prime}}{\delta^{\prime}}\right] \theta^{\prime}}=-\theta \\
t_{i}^{0}=\frac{\tau_{\infty}}{\mathrm{f}\left(\eta_{\mathrm{m}}\right)} \delta_{\theta}^{e} \frac{\mathrm{d} \theta}{\left[\exp \left(\frac{\theta}{1+\beta \theta}\right)-\frac{\theta}{\delta}\right]}
\end{gathered}
$$

Looking first at eq. $B-39, t_{q}^{\circ}$ can be estimated ${ }^{+}$for $-\theta$ in much greater than 2 as:

$$
\begin{gathered}
t_{q}^{0} \sim \frac{\tau_{\infty}}{f\left(\eta_{m}\right)}\left\{\int_{0}^{2}-\frac{d \theta^{\prime}}{\left[1+\left(a_{1}-\Delta\right) \theta^{\prime}+a_{2}\left(\theta^{\prime}\right)^{2}\right]}+\delta^{\prime} \stackrel{2}{\sim}\left(\frac{-\theta}{2}\right)-\left(\delta^{\prime}\right)^{2} E\right\} \quad B-41 \\
-\theta_{\text {in }} \gg 2, \varepsilon \leq 0.018, a_{1}=1.8628, a_{2}=0.2055
\end{gathered}
$$

Values of $t_{q}^{\circ}$ for $\theta_{\text {in }}=-10$ to $\theta=-2$ and $\theta=-2$ to $\theta=0$ are listed in the first two lines of table $B-1$ for $\Delta=0.5$ and $\Delta=0$. Warming of the sample from $\theta_{\text {in }}$ to -2 is accomplished almost completely by heat transfer from the environment. The value of $t_{q}^{0}$ for the latter temperature interval depends only on the thermal relaxation time (i.e. from eq. B-18, $\delta^{\prime} \tau_{\infty_{-}} / f\left(\eta_{m}\right)$ $\left.=\tau_{q}\right)$. Warming of the same from $\theta=-2$ to 0 involves both self heating and heat transfer; neglect of the former would result an infinite value of $t_{q}^{0}$ for this temperature interval. $t_{q}^{0}$ divided by $\tau_{\infty} / f\left(\eta_{m}\right)^{-}$decreases as $\Delta$ decreases because,-at constant $\tau_{\infty} / f\left(\eta_{m}\right)$, this requires that $\tau_{q}$ decreases (see again eq. B-18). Thus, the heat transfer to the sample increases, which shortens $t_{q}^{0}$.

${ }^{a_{1}}$ and $a_{2}$ are determined by fitting $\exp \left[-\theta^{\prime}-0.05\left(\theta_{\prime}^{\prime}\right)^{2}\right]$ with, a quadratic at $\theta^{\prime}=1^{2}$ and $\theta^{\prime}=2$. The error in the integral from $\theta^{\prime}=0$ to $\theta^{\prime}=2$ is $\sim 0.02 \tau_{\infty} / f\left(\eta_{m}\right)$. The value of $\varepsilon$ is $p(-2)$ where $p(X)$ is defined by Flynn and $\mathrm{Wall}^{\infty}$ (see $[6]$, eq. 13c). 
Table B-1

Induction Times and Reactant Consumption,

Explosive States, $f(n)$ is constant

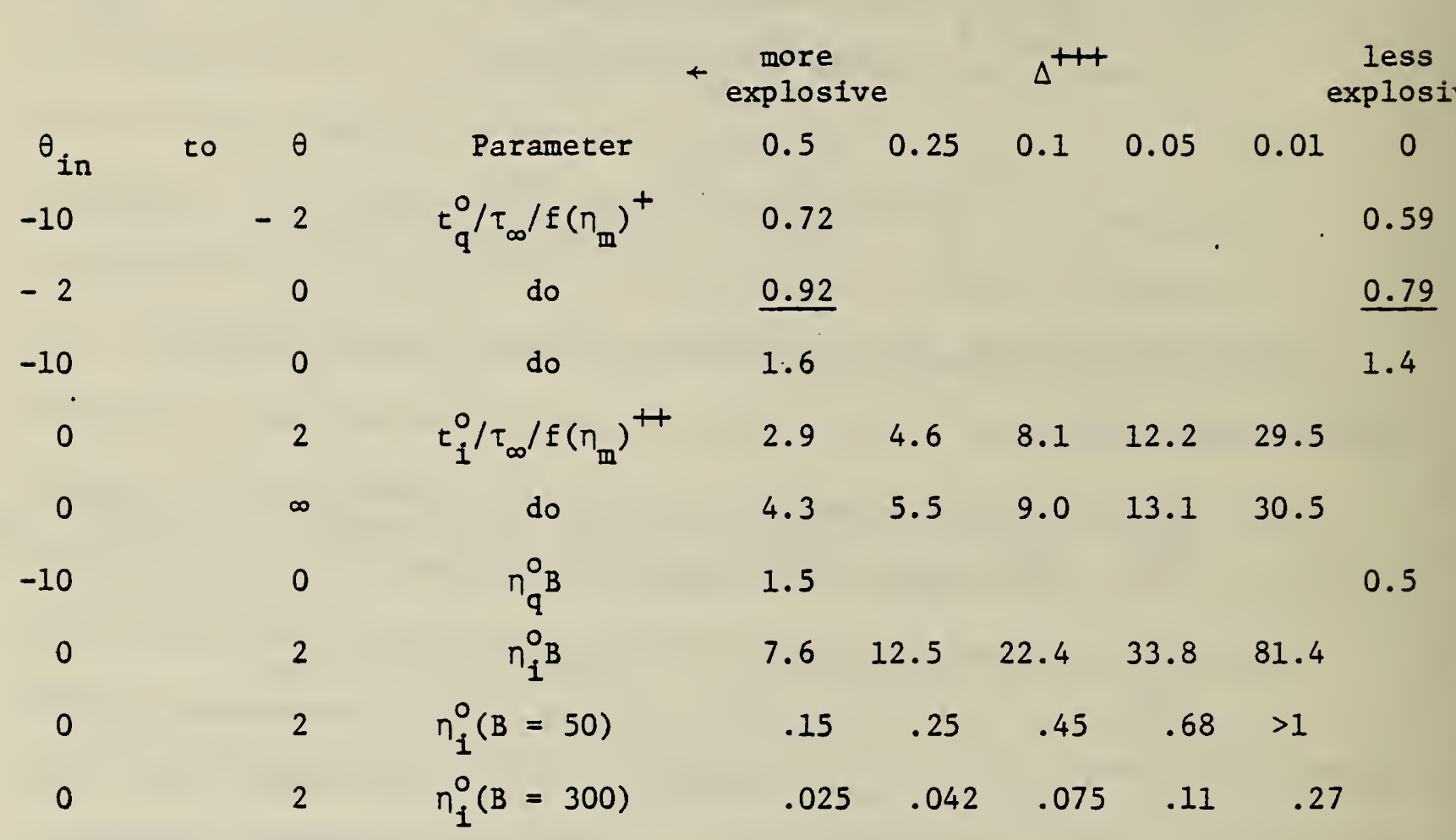

$\overline{t_{\theta_{\text {in }}} \ll-2}$

+ quadratic approximation

${ }^{+}+{ }_{\Delta}=\mathrm{e}-1 / \delta^{\prime}$ 
Using the quadratic approximation, one can estimate for $t_{i}^{\circ}$ :

$$
t_{1}^{0} \sim \frac{\tau_{\infty}}{f\left(\eta_{m}\right)} \frac{2}{Z}\left\{\tan ^{-1}\left(\frac{2 \theta}{e^{-Y}}\right)+\tan ^{-1}\left(\frac{\dot{Y}}{Z}\right)\right\} ; y=2-\Delta ; z=\left(4-y^{2}\right)^{1 / 2} \quad B-42
$$

When $\Delta$ becomes smal1 $+t_{i}^{0}$ becomes for $\theta_{e}$ greater than one:

$$
t_{i}^{\circ} \sim \frac{\tau_{\infty}}{f\left(\eta_{m}\right)} \frac{\pi}{\Delta}
$$

Numerical values of $t_{i} /\left(\tau_{\infty} / f\left(\eta_{m}\right)\right)$ for $\theta_{e}=2$ and $\theta_{e} \rightarrow \infty$ are listed in table $B-1$ in the fourth and fifth lines, respectively. The latter set of values approaches the value of eq. $B-43$ as $\Delta$ decreases.

As the state gets less supercritical (i.e. as $\Delta$ decreases) $t_{i}^{0}$ becomes progressively less dependent on the exact value of $\theta_{e}$ (compare corresponding columns of the fourth and fifth lines) as eq. B-44 predicts. $t_{i}^{0} /\left(\tau_{\infty} / f\left(\eta_{m}\right)\right)$ increases as $\Delta$ decreases for the same reason that $t_{q}^{0} /\left(\tau_{\infty} / f\left(\eta_{m}\right)\right)$ decreases. It should be noted that $t_{q}^{0} / t_{i}^{0}$ also decreases as $\Delta$ decreases; the statement in the literature that $t_{q}^{0} / t_{i}^{0}$ is negligible applies only when $\Delta$ is small regardless of how big $\theta_{e}$ may be. At $\Delta=0.5$, the minimum error in assuming $t_{q}^{\circ} / t_{i}^{0}$ to be zero is 38\%. The value of $\Delta=0.5$ actually corresponds to only a $7 \mathrm{~K}$ increase ${ }^{+}$above the critical bath temperature, $T_{*}$, in the explosion temperature test described in section $B .1$, if $T_{*}$ is $500 \mathrm{~K}$ and $E=35 \mathrm{kcal} / \mathrm{mol}$. It should be noted that if the time to explosion is divided into two parts and one of these is computed for the temperature range where self-heating is negligible, then this range must

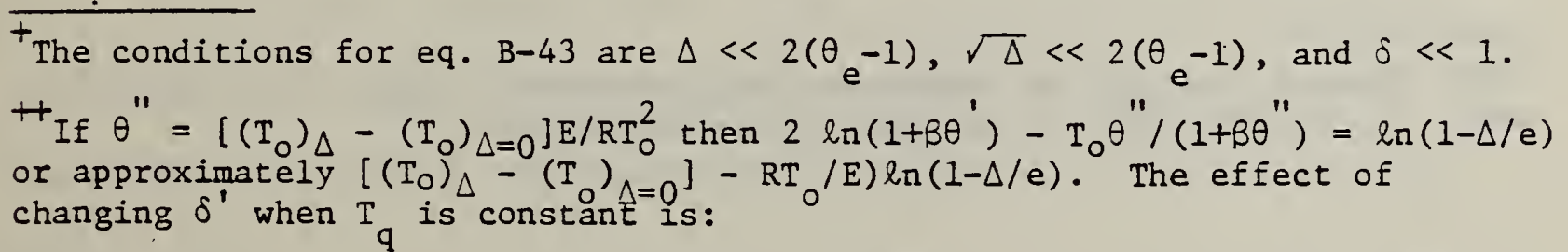

$\begin{array}{ccc}\Delta & {\left[\left(\mathrm{T}_{0}\right)_{\Delta}-\left(\mathrm{T}_{0}\right)_{\Delta=0}\right]} & \left(\tau_{\infty}\right) /\left(\tau_{\infty}\right) \Delta=0 \\ 2 & 45 \mathrm{~K} & 0.26 \\ 1 & 16 \mathrm{~K} & 0.63 \\ 0.5 & 7 \mathrm{~K} & 0.82\end{array}$


be below $\theta=-2$. Thus, boundary conditions of the form $\eta=0$ at $\theta=0$ do not approximate reality well and the error in this approximation is greater as $\Delta$ increases in the explosion temperature test.

Times to explosion of different materials can be correlated to a good first approximation by the comparing them at the same value of $\delta$ ' and $\theta_{\text {in }}$. Then the times to explosion are proportional to the thermal relaxation time, $\tau_{q}$. (From eq. B-18 $\tau_{\infty} / f\left(\eta_{m}\right)$ equals $\tau_{q} / \delta$ ', so each term in the right side of eq. $B-40$ and eq. $B-41$ is proportional to $\tau_{q}$ ) Since from eq. B-31, we have

$$
\tau_{q} \sim \frac{c r^{2} g_{2}}{\lambda g_{1}}\left(1+\frac{g_{1}}{B i}\right.
$$

the product of $t^{\circ} \alpha_{0} /\left(c g_{2}\right)$ should be the same at small Bi or $t^{\circ} \lambda /\left(\mathrm{cr}^{2} \mathrm{~g}_{2}\right)$ should be the same at large $\mathrm{Bi}^{+}$. $\mathrm{c}$ is the heat capacity per unit volume of material.

The values of $t^{\circ}$ calculated from eq. $B-41$ and eq. $B-42$ are lower bounds on the actual values of $t^{\circ}$ when $f(n)$ is changing according to an order decomposition mechanism provided the value of $\theta_{e}$ corresponds to a value actually reached by the material before $d \theta / d t=0$ when $f(\eta)$ is not constant. Since $\dot{t}_{i}^{0}$ equals $\tau_{\infty} \theta_{e} /\left[\left(1-\theta_{e}\right) f\left(\eta_{m}\right)\right]$ when $\Delta=0, \theta_{e}=\theta_{c}$ will be less than one when $1 /^{\prime}=$ e if $f(\eta)$ is not constant. From the material reviewed in the following section (see in particular fig. Bthis limits the utility of eqs. $B-41$ and $B-42$ to values of $\Delta$ (and $B$ ) that are not too small.

\footnotetext{
This assumes that all the materials are continuous solids, and, that up until $\theta_{e}$, heat transfer is by heat conduction alone.
} 
With this restriction in mind, one can use the corresponding expressions for $\eta^{\circ}$ to estimate upper bounds for the amount of initial material that has decomposed prior to an explosion in the case where $f(n)$ is not constant. From eq. B-14 one has letting $\psi=\theta /(1+\beta \theta)$

$$
\frac{d \eta}{d t}=\frac{d \eta}{d \theta} \frac{d \theta}{d t}=f\left(\eta_{m}\right) k_{T_{0}} \exp (\psi)
$$

Substituting eq. B-15 into the left side, rearranging, and integrating one has

$$
\eta^{\circ}=\frac{1}{B} \int_{\theta}^{\theta} \frac{\exp (\psi) d \theta}{\exp (\psi)-\theta / \delta^{\prime}}=\eta_{q}^{0}+\eta_{i}^{0}
$$

where $\eta_{q}^{\circ}$ is the reactant consumed during the time $t_{q}^{0}$ and $\eta_{i}^{0}$ is the reactant consumed during the time $t_{i}^{\circ}$. An estimate of $\eta_{q}^{\circ}$ for $-\theta_{\text {in }} \gg 2$ is

$$
\eta_{q}^{\circ}=\frac{1}{B}\left[\int_{0}^{2}\left[1-\frac{(e-\Delta) \theta^{\prime}}{\left[1+\left(a_{1}-\Delta\right) \theta^{\prime}+a_{2}\left(\theta^{\prime}\right)^{2}\right]}\right] d \theta^{\prime}+\delta^{\prime}(0.50)\right]
$$

where $a_{1}$ and $a_{2}$ are the same as for eq. $B-41$.

Using the quadratic approximation, $\eta_{i}^{0}$ can be estimated as

$$
\eta_{i}^{\circ}=\frac{1}{B}\left\{\theta_{e}+\frac{(e-\Delta)}{2} \ln \left[\theta_{e}^{2}+(\Delta-2) \theta_{e}+1\right]+\frac{(2-\Delta)(e-\Delta)}{2} t_{i}^{0}\right\} \quad B-45 a
$$

which for small $\Delta$ becomes

$$
\eta_{i}^{\circ} \sim \frac{1}{B}\left\{\theta_{e}+e \ln \left(\theta_{e}-1\right)+\frac{e \Pi}{\sqrt{\Delta}}\right\}
$$

Numerical values of $\eta_{q}^{\circ}$ and $\eta_{i}^{\circ}$ divided by $B$ are listed in the fifth and sixth lines of table $B-1$ for $\theta_{e}=2$. The numerical values of $\eta_{i}^{0}$ for $B$ $=50$, (a cellulosic material) and $B=300$ (an explosive) are tabulated in the last two lines. It is evident that $\eta_{q}^{\circ} / \eta_{i}^{\circ}$ for small $\theta_{e}$ is not negligible for large $\Delta$-an assumption of analyses that use the initial

\footnotetext{
$t_{\text {Equation } 3-42}$, which involves the quadratic approximation, yields 1.3 $\tau_{\infty}$ for $\theta_{e} \gg 1$ and $\Delta=e$ because the quadratic approximation underestimates $\exp (-E$ ?RT).
} 
conditions of $\eta=0$ at $\theta=0$. Also the statement that reactant consumption is negligible prior to an explosion applies only to explosives (i.e. when $B$ is large) and even then only when $\theta_{e}$ is small.

$\tau_{\infty}$ is called the adiabatic induction time because if $1 / \delta=0$ (i.e. $h^{\prime}=0$ or $1 / \tau_{q}=, 0$ ) then eq. $B-40$ becomes for an order reaction (i.e. $\left.f\left(n_{m}\right)=1\right)($ see $[13])$.

$$
\begin{aligned}
t_{1}^{0} & =\tau_{\infty}\left[1-\exp \left(-\theta_{e}\right)+2 \beta\left[1-\left(\theta_{e}+1\right) \exp \left(-\theta_{e}\right)\right]+\beta^{2} \ldots\right] \\
& =\tau_{\infty} ; \theta_{e} \gg 1
\end{aligned}
$$

It is to be noted if at the temperature $T_{0}$, the material is isolated from its environment, $n_{i}^{0}$ is decreased to $\theta_{e} / B$ because of of the heat generated by the decomposition goes into heating the sample. On the other hand; $\eta_{\mathrm{q}}^{0}$ is now increased to $-\theta_{\text {in }} / \mathrm{B}$ if $1 / \delta^{\prime}$ is zero from zero time onwards because heat absorption form the environment has been eliminated.

The dependence of induction times on the definition of the temperature, $\theta_{e}$, when an explosion takes place is mathematically somewhat arbitrary although $t^{\circ}$ becomes essentially independent of $\theta_{e}$ so long as it is much larger than one. In practice, explosions are detected by rupture of a container or some other physical event which corresponds to a temperature when $\mathrm{d} \theta / \mathrm{d}$ t or $\theta$ becomes large due to self-heating. One can see that a large $d \theta / d t$ always requires $\theta_{e}$ be greater than one regardless of how explosive a state the material is in (i.e. whether $\Delta$ is small or near e) from table $B-2$. This is in true in spite of the fact the feature of a $\theta$ versus $t$ curve that distinguishes explosive from subcritical states, an inflection, moves to progressively smaller values of $\theta$ well below zero as $\Delta$ increases. (The inflection temperature occurs at $\theta \sim 1+\ln (1-\Delta / e)$. The above criteria for $\theta_{\mathrm{e}}$ are meaningless when the material is an adiabatic condition, as the last column of table B-2 shows, because the material always explodes. 
Table B-2

$\frac{d \theta}{d t} \times\left[\frac{\tau_{\infty}}{f\left(n_{m}\right)}\right]$ versus $\theta ;$ Exponential Approximation

\begin{tabular}{ccccc}
$\theta$ & \multicolumn{3}{c}{$\Delta^{+}$} & e \\
-3 & 8.20 & 1 & 2.20 & .05 \\
-2 & 5.57 & 3.20 & 1.57 & .13 \\
-1 & 3.09 & 2.09 & 1.09 & .37 \\
0 & 1 & 1 & 1 & 1 \\
1 & 0 & 1 & 2 & 1.72 \\
3 & 1.95 & 3.95 & 5.95 & 7.39 \\
4 & 11.9 & 14.9 & 17.9 & 20.1
\end{tabular}

$\bar{t}_{\Delta=e-1 / \delta}$. 
When $\Delta$ is large, the simple model breaks down because, as Zinn and Mader [33] and Merzhanov (see [14] p. 280), have shown; the material at the container-sample interface reaches a critical condition even though the spatial or volume average temperature of the material may be very much less than $T_{0}$. This phenomenon, transition to the ignition regime, was mentioned in section B.1. Thus, it is to be expected that neither the formula for $t_{i}^{0}$ or $\eta_{i}^{0}$ will be valid when $\Delta$ is large. The effect of $f\left(\eta_{m}\right)$ on $t^{0}$ is different for order reactions where $f(0)=f\left(\eta_{m}\right)$ and autocatalytic reaction where $f(0) \ll f\left(\eta_{m}\right)$. $t^{0}$ will be much larger for autocatalytic reactions when reactant consumption is included in the model. Moreover, if the material is preheated and then again cooled, the time to explosion on subsequent to exposure to a high value of $T_{0}$ is increased for decomposition by an order reaction but is decreased for an autocatalytic mechanism. 


\section{B.5 Non Stationary Analysis of the Isothermal Model, $f(n)$ Variable}

From the previous two sections, which summarize the model for a zero order reaction mechanism one can identify the following criteria for distinguishing explosive from non-explosive states of the material:

(a) In non-explosive states, the temperature reaches a steady state slightly above the ambient temperature while in explosive states the temperature "takes off" after reaching this steady state value.

(b) At the critical boundary between explosive states and nonexplosive states, the material is in a metastable situation such that only a slight perturbation of initial conditions (i.e. amount of material, ambient temperature, or heat exchange with the environment) can shift the state of the material from an explosive to a non-explosive state or vice versa.

(c) Explosive states of the material have an inflection point in the $\theta$ or $\mathrm{T}$ versus $t$ curve before $\theta$ reaches its maximum value while non-explosive states do not.

When reactant consumption is now incorporated into the simple model, criterion (a) disappears, mathematically. This loss seems to have been a source of considerable concern to investigators in the field of thermal explosions because it raises doubt about any analysis of criticality that depends upon criterion (a) (all of the steady state analyses). For example, one finds on page 126 of Gray and Lee's review [13], the upsetting statement "...is one of the most important results to have emerged from non-steady state theory since it rationalizes ${ }^{+}$the problem of defining a suitable critical preexplosion temperature rise when reactant consumption is important".

\footnotetext{
Underline is ours; the quote is a comment on the analysis of Adler and Enig (41) discussed later in this section.
} 
Another group of prominent investigators, Merzhanov and coworkers, summarize the problem more dramatically as follows. Based on the extensive analyses of nonstationary analyses made by them and their coworkers, they state (see [14], p. 283) "...there are no critical conditions in the mathematical sense and likewise there is no heating-up preceding explosion." ${ }^{+}$Merzhanov et al. do qualify these remaks by adding (somewhat contradictorily) that "...thermal explosion corresponds to a narrow region, roughly for values of $B$ between 100 and 1000 , and the critical conditions have a clear physical significance." Thomas [15] puts the loss of criterion (a) into a more practical perspective by pointing out that "...assuming a finite amount of reactant avoids the unreality of an infinite temperature rise" (if an explosion occurs) but now "...there is no strict distinction between supercritical and subcritical states which is not in some sense arbitrary...". The ambiguity in this distinction is more important when B is small (see comments concerning eq. B-45 in B.4.). However, if B is very small, say $20^{++}$, the maximum dimensionless temperature rise is so small that the practical (experimental) distinction is more of a qualitative than a quanitative problem anyway.

The main concerns of theoretical investigations can be viewed as attempts to answer the following: (a) What are the essential conditions for any proposed definition of criticality? (b) What definition of criticality duplicates the results of steady state theory when $B$ is large, independent of the form of $f(n)$ ? (c) Can a definition of criticality be devised that is derivable from instability considerations that also fulfills the condition mentioned in item (b)?

\footnotetext{
${ }^{+}$Our survey of more recent literature shows this to be demonstrably incorrect. For $Q / R \sim 400 \mathrm{Jg}^{-1} \mathrm{c} / \mathrm{P} \sim 1.5 \mathrm{Jg}^{-1} \mathrm{~K}^{-1}, \mathrm{E} / \mathrm{RT} \sim 25$ and $\mathrm{T}_{\mathrm{O}} \sim 600 \mathrm{~K}$. $\rho$ is the density.
} 
We shall summarize the investigations in terms of these questions in detail only for simple order reactions and will comment on a limited number of the earlier investigations we have studied. A more complete review of these earlier investigations are given by Gray and Lee (see [13] Chapter 5).

From steady state theory it was inferred that if $\delta^{\prime}$ is redefined, as we shall do throughout this section, as

$$
\delta^{\prime}=B k_{T_{0}} \tau_{q}=\frac{B \tau_{q}}{\tau_{I}}
$$

then the effect of reactant consumption on $\delta$ at criticality might be expected to be

$$
\delta_{c}^{\prime} \sim \frac{1}{e} \frac{\left(1+R T_{o} / E \cdots\right)}{f\left(\eta_{c}\right)}
$$

where $\eta_{c}$ is some critical value of the fraction of initial material that has decomposed. This cannot $^{+}$be derived from a Semenov diagram analysis in which $\dot{q}_{1}$ now varies with time (because $f(n)$ changes) by letting $\dot{q}_{1}=$ $\dot{\mathrm{q}}_{2}$ and $\mathrm{d} \dot{\mathrm{q}}_{1} / \mathrm{dT}=\mathrm{d} \dot{\mathrm{q}}_{2} / \mathrm{dT}$ at the critical value of $\eta, \eta_{c}$ as was done before. Arguments developed in earlier work based on eq. B-48 prove to be correct provided criticality is defined to be an inflection point in the $\theta, t$ curve. In fact, $B-48$ is identical to the condition that in an explosive state near the critical condition the inflection point in the $\theta, t$ curve occurs at $\theta_{c}=1$ assuming $\left[n /\left(\left(1-n_{c}\right) B\right)\right]^{1 / 2}$ is much less than one ( $n_{c}$ is the value of $\eta$ at $\theta=1$, $n$ is the order of the reaction, assumed here to be an integer). This can be derived from the condition that $\dot{d}^{2} \theta / d t^{2}=0$ for $d \theta / d t>0$ which requires in the exponential approximation that:

$$
\frac{d}{d \theta}\left[f(\eta) e^{\theta}\right]=\left[1-\frac{n}{1-\eta} \frac{d \eta}{d \theta}\right] f e^{\theta}=\frac{1}{\delta}
$$

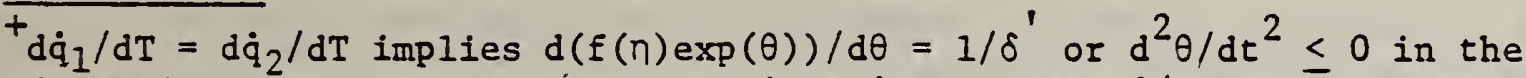
dimensionless time variable while $\dot{q}_{1}=\dot{q}_{2}$ implies $\mathrm{d} \theta / \mathrm{dt}=0$. These conditions are not simultaneously satisfied until all the material is decomposed and $\theta=0$.
} 
Noting that $d n / d \theta=\left\{B\left[1-\theta \exp (-\theta) /\left(f(n) \delta^{\prime}\right)\right]\right\}^{-1}$ one has letting $n=$ $n_{c}, \theta=\theta_{c}$

$$
(\mathrm{X}-1)\left(\mathrm{X}-\theta_{\mathrm{c}}\right)=n \mathrm{x}^{2} /\left[\left(1-\eta_{\mathrm{c}}\right) \mathrm{B}\right] ; \mathrm{x}^{-1}=\mathrm{f}\left(\eta_{\mathrm{c}}\right) \mathrm{e}^{{ }^{\theta} \mathrm{c}_{\delta}}{ }_{\mathrm{c}}^{\prime}
$$

The only value of $\theta_{c}$ that satisfies this relation and yields eq. B-48, when $R T_{o} / E$ and $\left[n /\left(\left(1-\eta_{c}\right) B\right)\right]^{1 / 2}$ are much less than one is $\theta_{c}$ equals 1 . An interpretation of this mathematics in terms of the Semenov diagram, modified for the case where $f(\eta)$ is not constant, is given later.

The argument developed by Frank-Kamenetskii (see [12] Chapter 7) to derive eq. B-48 illustrates the main physical ideas and assumptions of this earlier work (as well as their inconsistencies) and is outlined below.

Using the exponential approximation, the rate equation eq. B-14 becomes

$$
\mathrm{d} n / \mathrm{dt}=\exp (\theta)(1-n)^{\mathrm{n}_{\tau}}
$$

Assuming $\eta$ is small and $\exp (\theta)$ has an average value of e over the time prior to an explosion

$$
\mathrm{d} \eta / \mathrm{dt} \sim \mathrm{e}(1-\mathrm{n} \eta) / \tau_{r} ; \eta_{c}=\text { et }{ }_{c} / \tau_{r}
$$

where $t_{c}$ is the time to explosion when $\eta=0$ at $\theta=0$. Rearranging eq. $B-34$ where $\delta^{\prime}$ is now defined by $\in q$. B-47 we can estimate $t_{c}$ from

$$
t_{c} \sim \tau_{\infty} \int_{0}^{\theta} e \frac{d \theta}{\left[f\left(\eta_{c}\right) \exp (\theta)-\theta / \delta^{\prime}\right.}=\tau_{q} \int_{0}^{\theta} e \frac{d \theta}{\left[f\left(\eta_{c}\right) \delta^{\prime}(\exp (\theta)-0]\right.} \quad B-51
$$

In an explosive state near criticality, we have by analogy from steady state theory ${ }^{+}$

$$
f\left(\eta_{c}\right) \delta^{\prime} \sim(1+y) / e
$$

where $y$ is positive but small. Assuming $\theta=1+x$, one can approximate $\exp (0)$ if $X$ is small by

$$
\exp (\theta) \sim e\left(1+x+x^{2} / 2--\right)
$$

\footnotetext{
+Physically, the assumption that the material is an explosive state or nonexplosive state should not appreciably effect the value of $t_{c}$ very close to sriticality.
} 
Keeping terms to the first order of small quantities ( $x^{2}$ or $\left.X y\right)$ the expression $\mathrm{to}_{2} t_{c}$ becomes

$$
t_{c} \sim \tau_{q}^{\rho} \int^{\theta} e^{-1}\left[y+x y+x^{2} / 2 \cdots\right]^{-1} d x
$$

If $\left(\theta_{\left.e^{-1}\right) / y}\right.$ is large one obtains ${ }^{+}$

$$
t_{c} \sim \pi_{q} \frac{2}{y}
$$

Substituting into the expression for $t_{c}$ in eq. $B-50$ we have for small $y$ :

$$
\eta_{c}=e \pi \frac{\tau}{\tau_{r}} \sqrt{\frac{2}{y}}=e \pi \frac{\delta}{B} \sqrt{\frac{2}{y}}=\frac{e \pi(1+y)}{B e} \sqrt{\frac{2}{y}} \sim \frac{\pi}{B} \sqrt{\frac{2}{4}} .
$$

The parameter $\mathrm{y}$ is identified ${ }^{+}$by equating eq. $\mathrm{B}-48$ with the right side of eq. B-52a which gives for small $n_{c}$

$$
\frac{1}{e\left(1-n n_{c}\right)} \sim \frac{1}{e}(1+y) ; y \sim n n_{c}
$$

Substituting the value of $y$ into $B-53$ one obtains

$$
n_{c} \sim\left(\frac{2 \Pi^{2}}{n B^{2}}\right)^{1 / 3}=2.7\left(\mathrm{nB}^{2}\right)^{-1 / 3}
$$

Thus it is concluded from eq. B-48 that

$$
\delta_{c}^{\prime} \sim \frac{1}{e\left[1-2.7\left(\frac{n}{B}\right)^{2 / 3}\right]}
$$

The analysis by Thomas [34] makes the same type of assumptions + as those involved in obtaining eq. B-51 but departs in that the analogous differential form of eq. B-51 is converted to Airy's equation assuming y is small, and solved analytically for the value of $y$ that produces an inflection point in the curve. Thomas derives an equation of the same form

\footnotetext{
$t_{\text {Eq. }} \mathrm{B}-43 \mathrm{~b}$ yields the same value for $t_{c}$ except that 2 is replaced by $e$ because the quadratic approximation should produce a bigger $t_{c}$ than the exponential approximation. In either case eq. $B-52 b$ does not require that

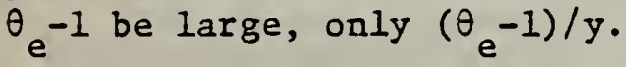

Hhis is an added assumption and is not deducible from the previous equations, in our opinion.

The quadratic rather than the exponential approximation is used. 
as eq. B-54b but with the constant 2.7 replaced by $2.85^{\dagger}$. The analysis of Rice et al (see [35], also [36,37] differs from that of Thomas mainly in that the analogous differential form of eq. B-52 is solved numerically (for a first order reaction). No formula analogous to eq. B-54 is derived. Examination of their results shows that an inflection point occurs very near $\theta=1$ and not at $\theta=2$ as stated by Gray and Lee ([13], p. 116).

The recent mathematical investigation of Gray and Sherrington (see [38] p. 448, fig. 2,3) determines directly the conditions for an Inflection point in the $\theta, t$ curve. The previous work reported by Tyler and Wesley [39] is essentially equivalent to determining an inflection point in the $\theta, t$ curve but is less direct.

It is useful to define the word "direct" because the associated procedure has been used to determine critical conditions in more recent non-stationary analyses: (1) The locus of inflection points (or any other criterion) is deduced by setting $d^{2} \theta / d t^{2}=0$. This yields a relation between $n, \theta$, and the dimensionless parameters $n$ (order of reaction), $B$, and $\delta^{\prime}$. (2) It is then required that the $\theta, \eta$ curve just touch and be tangential. to this locus at the single point of contact. This requirement is deduced by differentiating the locus of inflection points (or any other condition) with respect to $\theta$ and setting $d n / d \theta$ in this expression equal to that for the $\theta, n$ curve which is given by

$$
\mathrm{d} \eta / \mathrm{d} \theta=-\left[B\left[1-\exp (-\Psi) \theta /\left(\delta^{\prime} f(\eta)\right)\right]^{-1}\right.
$$

tour review of this work tentatively suggests that the analysis by Thomas could be improved by eliminating what appears to be an unnecessary assumption. Also, we do not see where $\theta_{c}=2$ at the inflection point is assumed to derive the critical condition which we infer Gray and Lee to state. 
where $\Psi=\theta /(1+\beta \theta)$ and $f(n)=(1-\eta)^{n}(n>0)$. (3) The values of $\theta=\theta_{c}$ and $\eta=\eta_{c}$ at which the $\theta, \eta$ curve first contacts and is also tangential to the integral curve can be determined (usually numerically) for any given $\mathrm{n}, \mathrm{B}$, and $\delta^{\prime}$. from eq. $\mathrm{B}-55$ and the locus of inflection points.

(4) The integral of eq. B-55, the so called integral curve, is then computed numerically for suitable initial conditions (usually $n=0$ at $\theta=0$ ) to determine if the curve intersects $\eta_{c}$ at $\theta_{c}$, etc.

To see what the direct procedure means graphically for a $\theta, t$ inflection point, a Semenov diagram appropriate to the case where $f(\eta)$ is not constant (only a single decomposition is taking place) has been constructed in figure B-2 for a first order reaction. $\dot{q}_{1}$ and $\dot{q}_{2}$ have been plotted in the vertical direction or $Z$ axis, $\theta$ on the $X$ axis, and $n$ (which varies from 0 to 1 ) in the $Y$ axis. The rate of heat generation, $\dot{q}_{1}$ in terms of eq. $B-15$ and $15 a$ can be described by

$$
\dot{\mathrm{q}}_{1}=\mathrm{B} \frac{\mathrm{d} \eta}{\mathrm{d} t}=\frac{B}{\tau_{I}} \exp \left(\frac{\theta}{1+\beta \theta}\right)(1-n)
$$

Thus, $\dot{q}_{1}$ is a curved surface that intersects the $x=0$ plane in the line $\dot{q}_{1}=\left(B / \tau_{r}\right)(1-\eta) \cdot \dot{q}_{2}$ the rate of heat loss, is then defined by

$$
\dot{q}_{2}=\frac{\theta}{\tau_{q}}=\frac{B \theta}{\tau_{r} \delta}
$$

$\dot{q}_{2}$ is a plane that has a slope $B /\left(\tau_{r} \delta^{\prime}\right)$ in the $Y=0$ plane and passes. through the $Y$ axis. The $\dot{q}_{2}$ plane intersects the $\dot{q}_{1}$ surface along the solid line I whose projection on the $z=0$ plane or $\theta, n$ plane is the line 1. Along $I$ or $i, d \theta / d t=0$ and this defines the maximum temperature the system can attain. Two integral curves (i.e. the integral of eq. B-55) have been plotted in the $Z=0$ or $X-Y$ plane as $J_{1}$ and $J_{2}$. Consider the meaning of the inflection in terms of these two integral curves. Assume $J_{1}$ is subcritical. Draw a vertical from any point on $J_{1}$ before its intersection 
$\dot{q}_{1}, \dot{q}_{2}$

(zaxis)

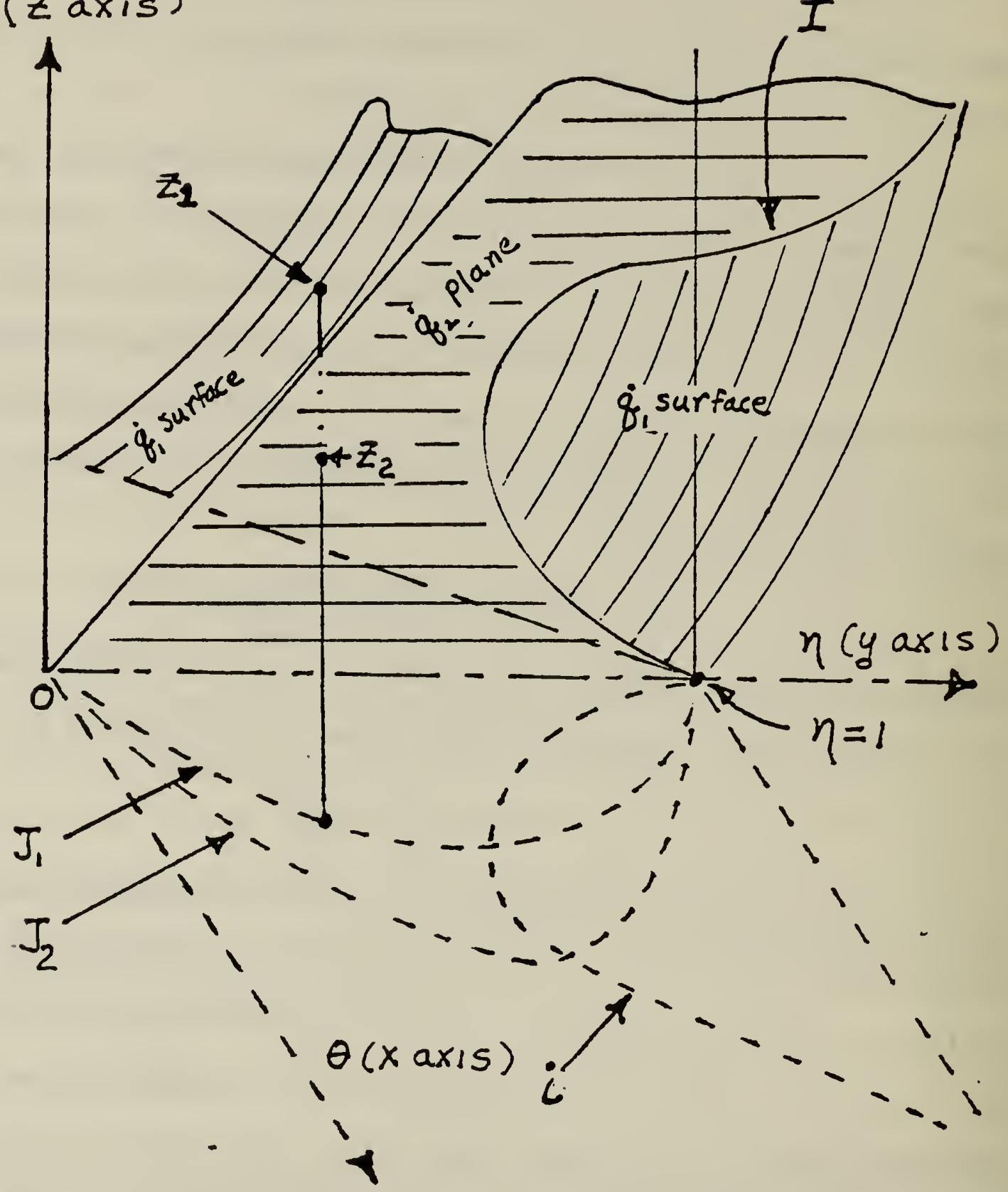

Figure B-2. Semenov Diagram; $f(n)=(1-\eta)$

$I$, intersection $\dot{q}_{1}$ surface and $\dot{q}_{2}$ plane; $i$, projection of $I$ on $z=0$ plane; $J_{1}, J_{2}$, integral curves; $Z_{2}$, intersection of vertical with $\dot{q}_{2}$ plane; $z_{1}$, intersection of vertical with $\dot{q}_{1}$ plane. 
with line 1 . It will intersect the $\dot{q}_{2}$ plane at the vertical height $\mathrm{z}_{2}$ and the $\dot{q}_{1}$ surface at the height $\mathrm{z}_{1}$. For the integral curve $J_{1}$ to be subcritical, the distance $z_{2}-z_{1}$ must always decrease as point defining the state of the system progresses along the $J_{1}$ curve from $\eta=0$ up to its intersection with line 1 . For the integral curve $J_{2}$ to be supercritical, the distance $\mathrm{z}_{2}-\mathrm{z}_{1}$ must decrease and then Increase as the point defining the state of the system moves along $J_{2}$ from $\eta=0$ to its intersection with the line 1 . The direct procedure outlined above shortens the procedure by determining, In step 1 , a line analogous to line 1 in the $X-Y$ plane which the integral curve must cut in order for $\mathrm{Z}_{1}-\mathrm{Z}_{2}$ to decrease and then increase. The remainder of the procedure constitutes finding that particular integral curve that is fust tangent to this inflection locus line.

There are other criteria for an explosion from a $\theta, t$ inflection point, however, and they are not less valid. As soon as one concedes that $f(n)$ is not a constant, one must redefine what constitutes an explosion either in terms of the character of the $\theta, t$ curve or the $\theta, n$ curve. Also, one need not use the direct procedure outlined above to determine critical conditions appropriate to any definition of an explosion. For example, Tyler and Wesley's [39] procedure differs from the direct procedure in that $d \theta / d t$ and $d n / d t$ are integrated numerically (initial conditions $\eta=0$ at $\theta=t=0$ ) for a given value of $n, B, B$, and a range of values $\delta^{\prime}$ as a function of time. Criticality for a particular set of $n, B$, and $\beta$ is identified as the value of $\delta^{\prime}$ which if increased by $1 \%$ doubles the maximum value of $\theta$ in the simple model or the maximum value of $\theta$ at the center of the material in the more complex models in which temperature gradients in the sample are taken into account. The critical value of $\theta$ is the value of $\theta$ (or $\theta$ in the center of the material) at $.99 \delta_{c}^{\prime}\left(.99 \delta_{c}\right)$. 
The equations for the simple model that were integrated by Tyler and Wesiey are as follows:

$$
\begin{aligned}
\frac{d \eta}{d \tau} & =\frac{\delta}{B}(1-\eta)^{n} \exp \left(\frac{\theta}{1+\beta \theta}\right) \\
\frac{d \theta}{d \tau} & =\delta(1-\eta)^{n} \exp \left(\frac{\theta}{1+\beta \theta}\right)-A \theta \\
& =\delta\left[(1-\eta)^{n} \exp \left(\frac{\theta}{1+\beta \theta}\right)-a \theta\right]
\end{aligned}
$$

These are written down because they are the ones most commonly used except in the Russian literature for comparison of the results of the simple model with those of models in which the material has a simple symmetrical geometry (sphere, infinite cylinder, or infinite slab), the boundary conditions are uniform and the temperature gradient varies in only one direction. The translation of the notation of eqs. B-56 and B-57 to ours is as follows

Eqs. B-56, B-57

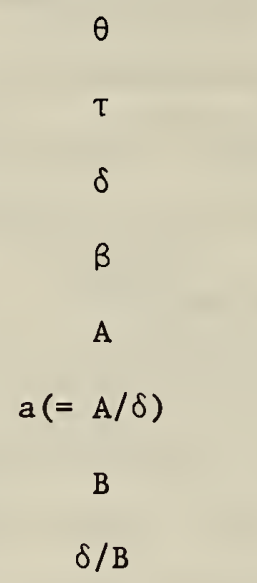

\section{Our notation}

$\theta$

$t /\left(\mathrm{cr}^{2} / \lambda\right)$

$\delta / f\left(n_{m}\right) ; \delta$ is in eq. B-31

B

$\mathrm{h}^{\prime} \mathrm{r}^{2} /(\mathrm{V} \lambda) ; \mathrm{g}_{1} / \mathrm{g}_{2}$ if $\mathrm{Bi}=\infty_{\infty}+$

$1 / \delta^{\prime}$; see eq. $\mathrm{B}-47^{++}$

B

$\operatorname{cr}^{2} \mathrm{k}_{\mathrm{T}_{\mathrm{o}}} / \lambda=\operatorname{cr}^{2} /\left(\lambda \tau_{\mathrm{r}}\right)$

The most important result of Tyler and Wesley is that the numerical values of $e / g_{2}$ for $B \geq 25$ and $\beta<0.1$ agree within $3 \%$ of those computed using the values of $1 / g_{2}$ cited previously in section $B .3$ for the more complex models of the infinite slab, infinite cylinder, and sphere cited in section B.3. In other words, the critical conditions can be evaluated

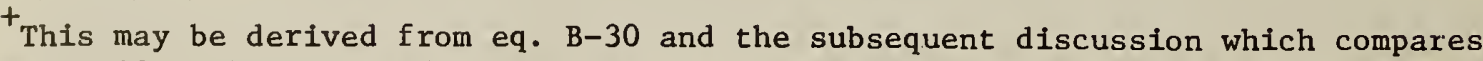
eq. B-30 with the steady state results of the same complex models and the simple mode1.
}

${ }^{+} \delta^{\prime}$ of this section is $\delta^{\prime}$ of $B .3$ and $B .4$ divided by $f\left(\eta_{m}\right)$. 
without taking into account the temperature gradients in the sample ${ }^{+}$. (If $\delta^{\prime}>\delta_{c}^{\prime}$ one cannot, however, talk about when an explosion takes place because the phenomena of the ignition regime occur as described in section B.4. Also the simple model cannot be used if the environment temperature is non uniform) ${ }^{++}$.

Numerical values of $\delta_{c}^{\prime}$, or $\delta_{c}$ for these more complex models, were within $0.2 \%$ of the corresponding critical value for an inflection point in the $\theta, t$ curve with $B \geq 100$ and $1.5 \%$ of the value at $B=25$. No critical condition (in their definition) occurs for $B \leq 14$ at $\beta=0$ and $B \leq 25$ at larger $\beta$, though in some of the cases inflections in the $\theta, t$ curve did occur. They found their numerical values for $\left(e \delta_{c}^{\prime}\right)^{-1}$ could be fitted to an expression of the form eq. B-54 (i.e. $\left(e \delta_{c}^{\prime}\right)^{-1}=a_{1}+a_{2}(n / B)^{2 / 3}$ within $2 \%$ for $B \geq 25$ for $n=1$ and $B \geq 100$ for $n=2^{H+}$ with values of $a_{1}$ near 1 and $a_{2}$ near 2.4. The value of $\theta_{c}$ could be expressed to within $3 \%$ by an expression of the form $a_{3}+a_{4}(n / B)^{2 / 3}$ where $a_{3}$ is near one and $a_{4}$ is between 5 and 13. Numerical values of $a_{1}$ through $a_{4}$ given by Tyler and Wesley are listed in table B-3 along with the other data relevant to this review. For example, $\theta_{c}$ for the various geometries do not differ greatly from $\theta_{C} \sim 1$ of the simple model when $n=0$ but they increase with increasing $\mathrm{n} / \mathrm{B}$.

Induction times to explosion are continuous as $\delta^{\prime}$ increases from below to above $\delta_{c}^{\prime}$, in contrast to the behaviour of the discussion in section B.4., and reactant consumption becomes very large as B gets small, in agreement with the qualitative argument of section B.4.

\footnotetext{
That is, correction for the effect of temperature gradients can be accomplished using the arguments leading to eq. $B-30$ and $B-31$ in steady state theory.
}

H non uniform ambient temperature would require that temperature gradients be taken into account explicitly rather than by the aposteriori approach leading to eq. B-30 and eq. B-31. 
Table $B-3$

Results of Tyler and Wesley [39]

$$
(n=1,2 ; B \geq 25 ; B 1=\infty ; n=0 \text { at } \theta=0)
$$

$$
\underbrace{(\delta e)^{\prime}}=a_{1}-a_{2}(n / B)^{2 / 3} ;
$$

$\begin{array}{lcc}\text { B } & a_{1} & a_{2} \\ .00 & 1.000 & 2.28 \\ .025 & 0.973 & 2.35 \\ .050 & 0.944 & 2.41 \\ .075 & 0.916 & 2.49 \\ .100 & 0.885 & 2.56\end{array}$

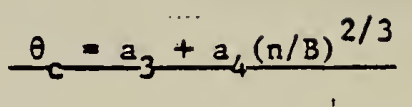

$\begin{array}{ll}a_{3} & a_{4} \\ 0.880 & 5.58 \\ 0.930 & 6.54 \\ 0.985 & 7.93 \\ 1.045 & 9.98 \\ 1.110 & 13.1\end{array}$

\section{Steady State Calculations}

$\begin{array}{lcc}\text { geomerty } & 8_{1} /\left(g_{2} e\right), \text { see eq. } B-30 & \theta_{c}^{\prime}(0)^{+} \\ \text {slab } & 0.8783 & 1.164 \\ \text { eylinder } & 2.000 & 1.376 \\ \text { sphere } & 3.3218 & 1.585\end{array}$

$+{ }^{\prime}{ }_{c}^{\prime}(0)$ is critical value at center of material of steady state analysis; for nonstationary analysis it is calculated from $\theta_{c}^{\prime}=\theta_{c} \theta_{c}^{\prime}(0)$.

Induction Times

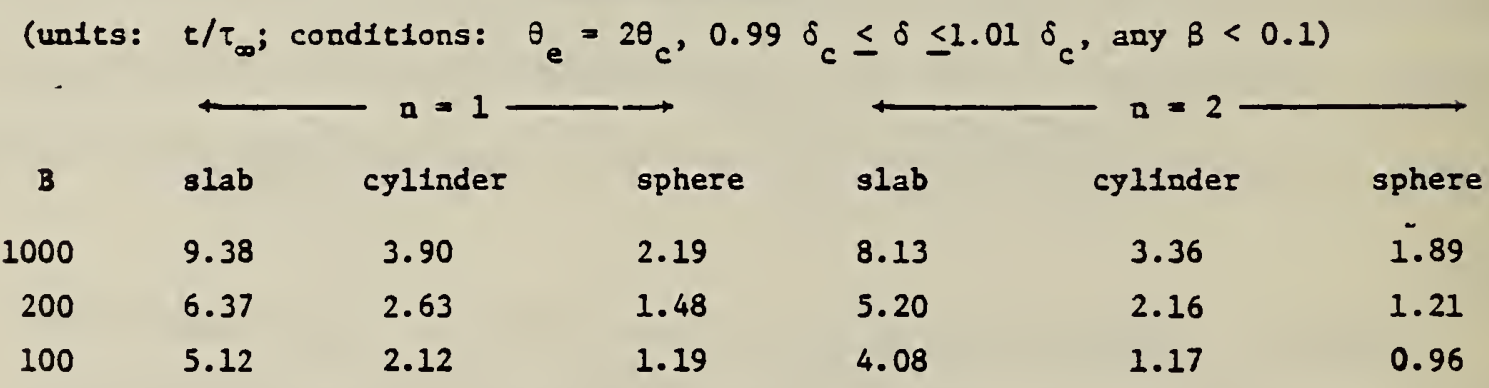

Percent Reactant Consumption in Sphere

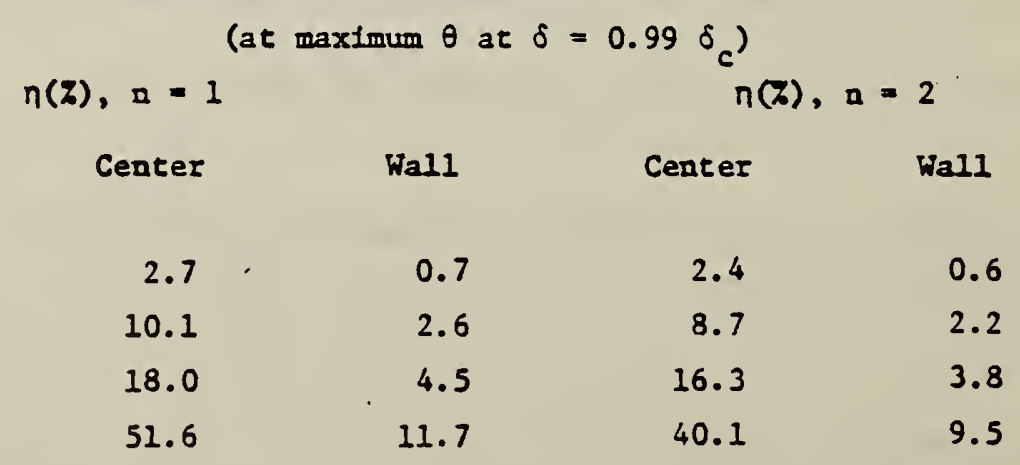


Gray and Sherrington, using the "direct" procedure (see [40], p. 438) showed that as $Y=1 / B$ gets small for $n=1$ and $\beta=0$, that $\theta_{c}$ approaches 1 at the critical condition for an inflection in the $\theta, t$ curve prior to the maximum value of $\theta$ in the $\theta, t$ curve.

Adler and Enig [41] have deduced the critical conditions for an inflection point in the $\theta, n$ curve by the direct procedure for $\beta=0$ and $n$ $=1,2,3,4,5$, and 10 . They have shown that criticality in this sense requires and necessarily implies that an inflection in the $\theta, t$ curve has already occurred if there is an inflection in the $\theta, n$ curve. Physically, this means that the subcritical region in the $a=1 / \delta^{\prime}$ versus $\gamma$ plane is larger for their criterion than for a $\theta, t$ inflection, as Gray and Sherrington (see $[40]$, p. 448) have pointed out.

A comparison of $a=1 / \delta^{\prime}$ versus $\gamma$ results of Tyler and Wesley, Gray and Sherrington, Adler and Enig, and the earlier results of Thomas are given in curves (a), (b), (c) and (d), respectively of figure B-3 for a first order reaction. The curve (a) from Tyler and Wesley is for the range of validity of their formula $(\gamma \leq 0.04)$ and the computed curve from Thomas, (d), is also valid only for small $\gamma$. (Curves (e) and (f) are discussed later. Below $\gamma=0.05$ curves (a) through (c) are essentially indistinguishable and their difference from curve (d) is remarkably small considering the approximations Thomas made in his analysis ${ }^{+}$. The agreement between curves (b) and (c) in this range is to be expected because Tyler and Wesley noted that according to their criterion of criticality $\delta_{c}^{\prime}$ occurs approximately when the maximum value of $\theta$ is 2 which is the requirement on $\theta_{c}$ of Adler and Enig's criteria for an inflection point in the integral

\footnotetext{
We estimate that the net error in the approximations made by Thomas to convert the right side of $\mathrm{B}-15 \mathrm{a}$ to a form that can be integrated analytically is of the order of $20 \%$ or so. In this sense, reasonable agreement should be expected.
} 


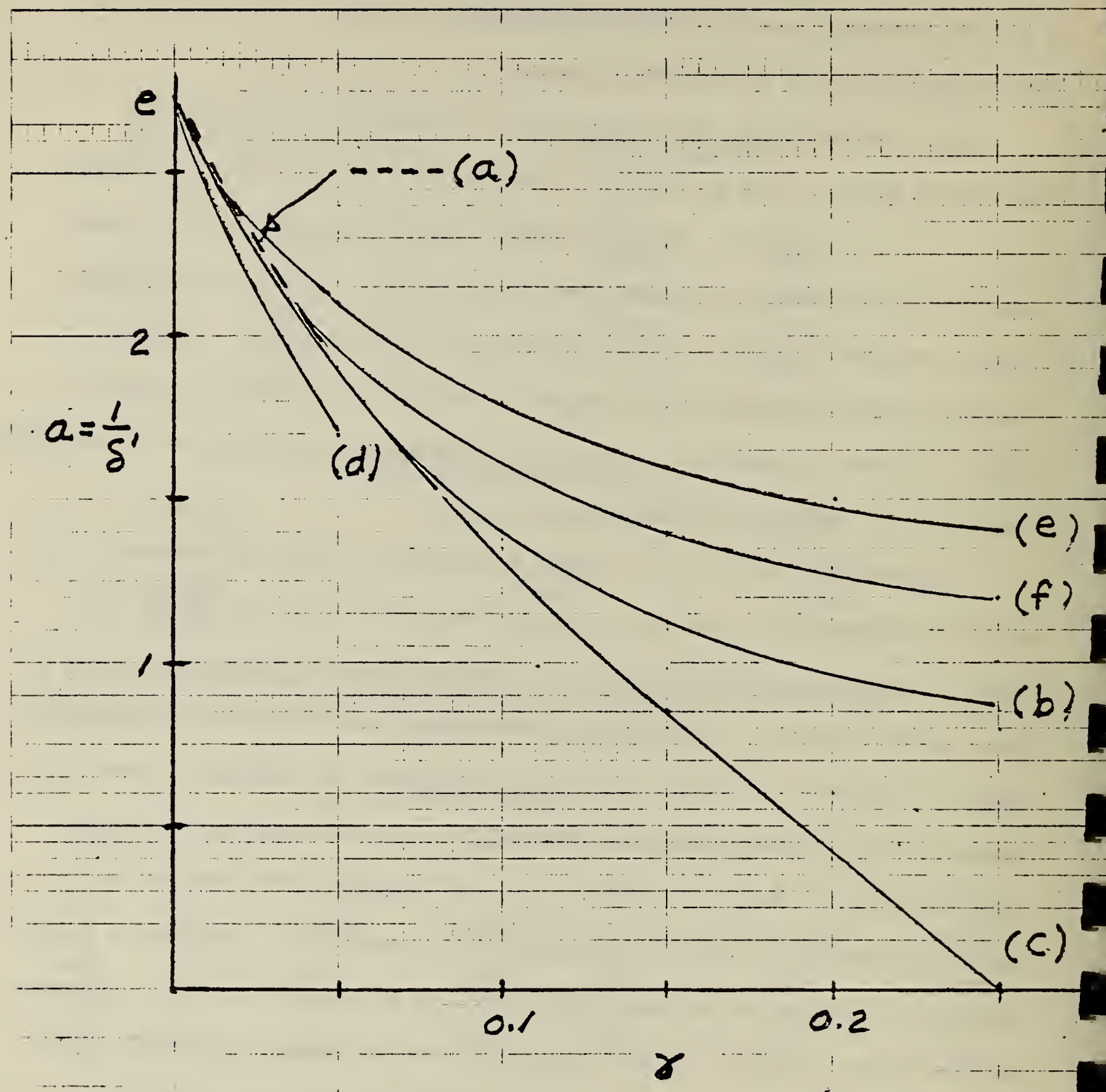

Figure $B-3$. Sketch of $1 / \delta^{\prime}=$ a versus $\gamma=1 / B, n=1, \beta=0$

(a) Tyler and Wesley. [39]; (b) $d^{2} \theta / d t^{2}=0$, Gray and Sherrington [40];

(c) $d^{2} \theta / d n^{2}=0$, Adler and Enif [41]; (d) $d^{2} \theta / d t^{2}=0$, Thomas [34];

(e) First trial function, Gray et al [38]; (f) Second trial function, Gray et al [38].

B. 5-14 
curve when $\mathrm{n}=1(\beta=0)^{+}$. However, Tyler and Wesley noted that their value of was within a few percent of that required to first produce $a, t$ inflection point. Hence the agreement is not coincidental.

Outside the range $\gamma \leq 0.05$ curves (b) and (c) diverge rapidly. Further, curve (a) cannot be realistically extrapolated because Tyler and Wesley's criterion for criticality is no longer valid. The reason for the latter statement can be seen from a rough sketch, figure B-4 of $\theta_{m}(\theta$ at $\mathrm{d} \theta / \mathrm{dt}=0$ ) as a function of $\delta^{\prime}$ for various $\gamma$ taken from Merzhanov et al [42] for $n=1, \beta=0$. For $\gamma=0.05$ it is still possible to obtain a large change in $\theta_{m}$ for a small change in $\gamma$ identifying, thus, a critical value of $\delta_{c}^{\prime}$. Also, the magnitude of $\theta_{m}$ for a large value of $\delta^{\prime}$ is quite large. However, for $\gamma \geq 0.1, \delta_{c}^{\prime}$ by Tyler and Wesley's criteria becomes meaningless and the maximum value of $\theta_{m}$ at large $\delta^{\prime}$ gets progressively smaller. A representative value for the maximum temperature rise above ambient, $T_{m}-T_{0}$, is calculated for an activation energy of $30 \mathrm{kcaimol}^{-1}$ and $\mathrm{T}_{0}=600 \mathrm{~K}(327 \mathrm{C})$ to put the figure into a less abstract perspective. It is clear from figure B-4 that for $\gamma \geq 0.1$, Tyler and Wesley's criterion breaks down. It is for this reason that Merzhanov et al [14], for example, state that there is no critical condition for large $\gamma$.

The sketch in figure $B-4$ is too inaccurate ${ }^{+}$to determine $\theta_{m}$ corresponding to $\delta_{c}^{\prime}$ for $d^{2} \theta / d t^{2}=0$ and $d^{2} \theta / d n^{2}=0$ but some rough estimates can be made and they are shown by the dotted lines. It seems fairly clear (but this must be checked) that both of these criteria would not consider a maximum,

\footnotetext{
The criteria of Tyler and Wesley and Adler and Enig are not exactly the same. An inflection in the $\theta, n$ curve (for $n=1$ ) occurs whenever the integral curve passes thru $\theta_{c}=1$ and $1-n_{c}=2 \operatorname{aexp}(-2)+2 / B . \theta_{c}$ and $1-n_{c}$ are not the same as the maximum values of $\theta$ and $\eta$ at the maximum $\theta$ when $\mathrm{d} \theta / \mathrm{d} \eta=\mathrm{d} \theta / \mathrm{dt}=0$.

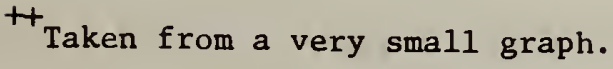




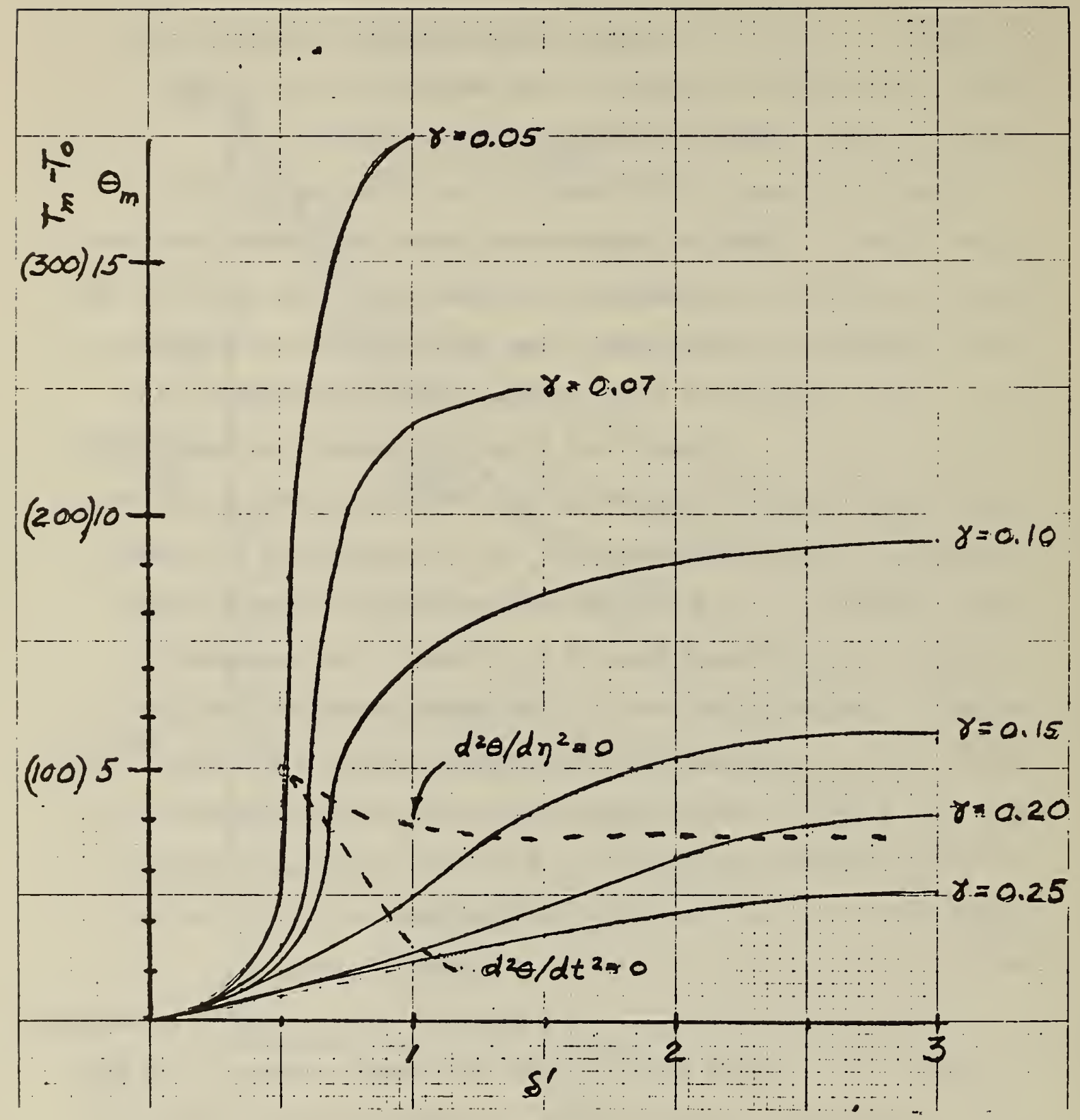

Figure B-4. Sketch of $\theta_{\mathrm{m}}\left(\theta\right.$ for $\mathrm{d} \theta / \mathrm{dt}=0$ ) versus $\delta^{\prime}$ (after Kerzhanov et al [42])

$\mathrm{T}_{\mathrm{m}}-\mathrm{T}_{0}$ for $\mathrm{E} / \mathrm{RT} \mathrm{T}_{\mathrm{o}}=25, \mathrm{~T}_{\mathrm{o}}=600 \mathrm{~K} ; \mathrm{n}=1, \beta=0$. 
$\theta_{m}$, of the order of 5 to be an explosion at $\gamma=.05$ and this would constitute a temperature rise above ambient of $100 \mathrm{C}$ if $\mathrm{T}_{0}$ is $600 \mathrm{~K}(327 \mathrm{C})$ and $46 \mathrm{C}$ if $\mathrm{T}_{0}$ is $373 \mathrm{~K}(100 \mathrm{C})$. Approximately $5 \%$ of the original material would be left by the time $\theta=\theta_{m}$ ! It seems relevant to point out that if the decomposition products were gases and the material is in a closed container an explosion would occur in the real world at least in terms of container rupture. Using the $\theta, n$ inflection criterion one finds that even at the time $\theta=2$, roughly one half of the sample is decomposed. Thus it is evident that an explosion criteria of the type being discussed is of primary interest to the shipping agent only if gaseous products are not liberated (i.e. this is an important part of the hazard rating that theory cannot take into account explicitly. Implicity, it is done through the statement $\eta \neq 0)$.

Recently, Gray and Sherrington [40], have started an investigation aimed at setting up an explosion criterion that has some physical foundation that is not purely intuitive. Their arguments, as we interpret them, starts from the second observation noted at the beginning of this section (see (b). of the first paragraph): 1) Because stability analysis alone can be used to deduce all the results of the steady state theory, it should be possible to deduce critical conditions when $f(n)$ is not constant by time dependent stability analysis. 2) The time dependent stability analysis should be selected so all the critical conditions of the steady state theory are generated as a particular case. Otherwise the limits of applicability of the results of the steady state results will not be known. 3) The preceding particular case or conditions are that in the limit when $\gamma=$ $1 / B$ goes to zero, $\theta_{c}=1$ and $\delta_{c}^{\prime}=1 /$. This corresponds to the experimental fact that as $\gamma \rightarrow 0$ it is found that the steady state results apply with $f(n)$ taken to be constant. 
Gray [43] has recently pointed out that if the third argument is

correct it should be true that our original time dependent equations must have the same critical conditions as those given in section B.3 in the limit when $\gamma=1 / B \rightarrow 0$. Referring to eq. B-14 and eq. B-15a one can write them as

$$
\begin{gathered}
\frac{1}{B} \frac{d \theta}{d t}=\gamma \frac{d \theta}{d t}=f(\eta) \exp (\psi)-\frac{\theta}{\delta} ; \psi=\frac{\theta}{1+\beta \theta} \\
\frac{d \eta}{d t}=\frac{f(n)}{\tau_{r}} \exp (\psi)
\end{gathered}
$$

The problem is then to prove that critical conditions for the solutions of the limiting case, $\gamma \rightarrow 0$ :

$$
\begin{aligned}
0 & =f(\eta) \exp (\psi)-\frac{\theta}{\delta} \\
\frac{d n}{d t} & =\frac{f(\eta) \exp (\psi)}{\tau_{r}}
\end{aligned}
$$

are $\theta_{c}=1$ and $1 / \delta_{c}^{\prime}=e$, for example, when $\beta=0$, for a first order reaction and $1 / \delta_{c}^{\prime}=e / 4$ when $\beta=0$ for a first order autocatalytic reaction. It is surprising but nevertheless true that this was proved only recently [44].

We are in disagreement with the idea that the last set of equations is the Semenov problem discussed in sections B. 3 and B.4, however. (We may be inferring something Gray did not mean.) The equations of B. 3 and B. 4 are those solely for inexhaustible reactant (or starting material which for large B make the change in $f(\eta)$ about some preselected value of $n=\eta_{m}$ very small. We see no reasons for replacing one unreal situation (inexhaustible reactant and preselected $f\left(\eta_{m}\right)$ ) with an equally unreal situation of inexhaustible reactant with zero relaxation time ${ }^{+}$.

$\overline{t_{\delta}^{\prime}=B \tau_{q} / \tau_{r}}=\tau_{q} /\left(\gamma \tau_{r}\right)$ for fixed $\delta^{\prime}$ and $\tau_{r}, \tau_{q} \rightarrow 0$ as $\gamma \rightarrow 0$ 
So far, results in regards to developing a stability analysis for critical conditions consistent with the three arguments advanced by Gray and Sherrington must be regarded as preliminary. A stability analysis using the direct procedure ${ }^{+}$of Liapunov with a Liapunov function that is quadratically dependent on the perturbations in $\theta$ and $\eta$, but otherwise is time independent, has been carried out [38] for a first order reaction for two forms of the quadratic. The results are shown as curves (e) and (f) of figure $B-3$; curve (f) is the better of the two estimates of the subcritical region using this type of Liapunov function. Recently Gray [45] has proposed a more restrictive stäbility criterion but no numerical results have been presented yet. It is, as of now, pure conjecture but it seems likely on physical grounds that stability analysis will yield a $a=1 / \delta$ versus $\gamma$ curve lying close to the $\theta, t$ inflection point curve.

\footnotetext{
This is not the direct procedure referred to previously. It involves the idea of, for example, guessing a function $V(E, S, t)$ where $E$ and $S$ are the perturbations in $\theta$ and $\eta$, respectively, such that $V$ is positive definite and $d V / d t<0$ for all $t$ as the locus of stability in step 1 of our direct procedure and then determining critical conditions.
} 


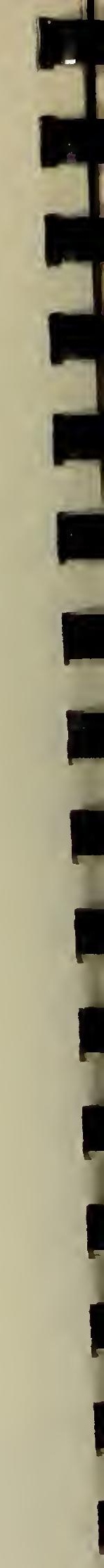




\section{B. 6 Temperature Gradients in Material, Various Geometries}

The heat balance equation assuming the material is a continuous solid, heat transfer is by conduction only, and the heat generated power unit volume under isothermal conditions is everywhere the same is

$$
c \frac{d T}{d t}=Q \frac{d \eta}{d t}+\lambda \nabla^{2} T
$$

All the symbols have been defined in section B.3-5 except $\nabla^{2}$, which is the Laplacian operator, and now $T$ is a function of position, $x$. For the infinite slab, infinite cylinder, and sphere

$$
\nabla^{2} T=\frac{d^{2}}{d x^{2}}+\frac{n d}{x d x}
$$

where $\mathrm{n}^{\prime}=0,1$, and 2 respectively. In dimensionless notation the heat balance equation becomes for these geometries

$$
\begin{gathered}
\frac{d \theta}{d\left(t / \tau_{\infty}\right)}=f(n) \exp \left[\frac{\theta}{1+\beta \theta}\right]+\frac{1}{\delta}\left(\frac{d^{2} \theta}{d \varepsilon^{2}}+\frac{n \dot{d} \theta}{\varepsilon d \varepsilon}\right) \\
\frac{d \eta}{d\left(t / \tau_{\infty}\right)}=\frac{1}{B} f(\eta) \exp \left[\frac{\theta}{I+\beta \theta}\right]
\end{gathered}
$$

where all the symbols have been defined previously (e.g., $\delta$ is defined by eq. B-31) except $\varepsilon$ which is $x / r$ where $r$ is the half width of the slab, or radius of the cylinder or sphere. For these geometries, the boundary conditions are:

$$
\begin{gathered}
\varepsilon=0 ; \partial \theta / \partial \varepsilon=0 \text { (i.e. center of symmetry) } \\
\varepsilon=1 ; \partial \theta / \partial \varepsilon=-B i \theta \text { (i.e. surface of material) } \\
t / \tau_{\infty}=0 ; \theta_{i n} ; \theta_{i n}=E\left(T_{i n}-T_{0}\right) / R T_{0}^{2} \leq 0 ; \\
\eta=0
\end{gathered}
$$

When $\mathrm{Bi} \rightarrow \infty$ the boundary condition at $\varepsilon=1$ is $\theta=0$ and the material is in perfect thermal contact, with the container. However, if $\mathrm{Bi} \rightarrow 0$ the heat loss term becomes

$$
\operatorname{Limit}_{\mathrm{Bi} \rightarrow 0} \frac{1}{\delta}\left(\frac{\partial^{2} \theta}{\partial \varepsilon^{2}}+\frac{\mathrm{n} \partial \theta}{\varepsilon \partial \varepsilon}\right)=\frac{\theta}{\delta}
$$


where again $\delta^{\prime}$ is defined in eq. B-31a. The simple model discussed in detail in sections B3.-B5. is the exact model (see [13], p. 64). (This is true for any geometry but does require that the rate of heat generation be the same everywhere in the material under isothermal conditions.) That is, as the heat loss becomes small, the temperature gradient in the material also becomes small. Physically, one can see if one has heterogeneous heat generation in the form of reactions at or around surfaces of particles, the same result will apply to the extent that the individual particle volume is small in comparison to the total volume of the material.

\section{Steady State Analysis $(d \theta / d t=0)$}

One can write the critical conditions in the form

$$
\begin{aligned}
& \theta_{c}=\theta_{c}{ }^{\circ} \phi_{\theta}(B i) \phi_{\theta}(\beta) \\
& \delta_{c}=\delta_{c}{ }^{0} \phi_{\delta}(B i) \phi_{\delta}(\beta)
\end{aligned}
$$

where $\theta_{c}{ }^{\circ}, \delta_{c}{ }^{\circ}$ are the critical temperature and critical FK (Frank-Kamenetski) parameter when $B i$ is infinite and $\beta=0$. The. $\phi^{\prime}$ 's are the corrections for finite $B i o t$ number and $\beta=\mathrm{RT}_{\mathrm{o}} / \mathrm{E}$, resfectively. Strictly speaking these $\phi$ corrections are a function of the specific geometry. Merzhanov et al [14], p. 281) have pointed out that to within $10 \%, \phi_{\delta}(B i)$ is the same for the infinite slab, infinite cylinder, and sphere (see footnote, p. B-17). They also indicate that, to a good approximation (no error limits stated), $\phi_{\theta}(\beta) \sim 1+2 \beta \ldots$ and $\phi_{\delta}(\beta) \sim 1+\beta \ldots$ in analogy with the simple model of section B.2.

A detailed discussion of exact values for $\delta_{c}{ }^{\circ}$ for the infinite slab, infinite cylinder, and sphere are given, respectively, in ref. [13] on pages 39,42 , and 44 respectively and values summarized on page 45 . The corresponding exact values of $\phi_{\theta}(B i)$ and $\phi_{\delta}(B i)$ are discussed in ref. [13] Q. 64-69. Exact values of $\delta_{c}{ }^{\circ} \phi_{\delta}(\beta=.01)$ are known for the curbe and regular circular cylinder - see table on page 63 of ref. [13]. 
Approximate methods of calculation of $\delta_{c}{ }^{\circ}$ for more complicated geometries are discussed in ref. [13] on pages $51-63$ and are summarized on page 63; a brief summary is found in ref. [14], p. 282 .

Methods of relating the critical parameters of the infinite slab, infinite cylinder, and sphere to those of the simple model are discussed in detail in ref. [13], p. 47 and p. 69-74. (Our extension of the simple model to these geometries is essentially the same as that of Barzykin and Merzhanov [46] - summarized on p. 73-74 of ref. [13]).

Assymetric boundary conditions for the infinite slab and infinite cylindrical annulus have been discussed in detail in ref. [13], p. 74-87.

A tabulation of more recent articles (i.e. since the publication of ref. [13], [14]) is given below:

More (or less) Exact Methods

$\underline{\text { Ref. }}$

1. Mathematical properties of steady state equations $(\beta \neq 0)$

2. $\infty$ slab (symmetric and asymetric boundary conditions)

3. $\infty$ slab (periodic variation of temperature with position).

4. Sphere, solid and hollow cylinder (heat flux prescribed at boundaries).

Approximate methods

5. Simple geometries $(B i=\infty)$

6. Slab, solid and hollow cylinder, solid and hollow sphere $(B i \neq \infty)$

7. Rectangular parallel piped, finite right cylinder, cone

$$
(B i=\infty)
$$

8. Arbitrary shape (arbitrary Bi) 
9. Forced convection (references to other papers)

10. Hot gas bubble in cool reacting liquid

\section{Non Steady State Solutions}

A detailed discussion of the earlier work (analytical and numerical) for the case where $f(\eta)$ is constant is given in ref. [13], p. 134-150. The discussion in ref. [14], though published at the same time, updates the discussion of ref. [13] in terms of the extensive advances made by Russian investigators. It includes the case where $f(\eta)$ is not constant, autocatalytic decomposition, temperature-time variations of the environment other than a step jump, convective heat transfer, etc. 


\section{B.7 Autocatalytic, Multiple, and Heterogeneous Reactions}

The rate expression given in eq. B-22 (see section B.3.) is the simplest phenomological representation of the catalysis of a reaction by a reaction product (see $[58])$. The reactions is self-accelerating under isothermal conditions but cannot explode as in the case of some branched chain reactions (see [12], chapter 1): $\eta_{0}$ must be of the order of 0.1 or less for the reaction be considered autocatalytic ${ }^{+}$; for chain reactions described by this macroscopic decomposition expression $n_{0} \sim 10^{-4}$ to $10^{-6}$ [59]. Both Gray and Lee (see [13], p. 151-167) and Merzhanov et al (see $[14]$, p. 284) have reviewed the earlier analysis of the heat balance and rate expressions for this form of $f(n)$.

Mielentiev and Todes [60] carried out an analysis of the problem using a method ${ }^{+}$involving the integral curve (i.e. $n$ vs. $\theta$ ) that yields results Identical to that of a Semenov diagram analysis (see section B. 3 ).

$$
\begin{gathered}
1 / \delta_{c}^{\prime}=e\left(1+n_{0}^{2}\right) / 4 ; n_{0} \ll 1 \\
\theta_{c}=1
\end{gathered}
$$

The method assumes the temperature dependence of $\eta_{0}$ can be ignored, which has been proved by Todes [61] (see [59], p. 1063). Merzhanov and

If $n_{0}$ is 1 , the reaction is effectively zero order from the point of view of a thermal explosion criterion.

H Gray and Lee [13] discuss this in detail. It is simpler to understand by using the method for $a$ first order reaction. The locus of $\mathrm{d} \theta / \mathrm{dt}=0$ is $1-\eta \sim\left(\theta / \delta^{\prime}\right) \exp (-\theta)$. A plot of this locus shows it intersects $\eta=0$ at two values of $\theta$ (one less than 1 and the other greater) if $\delta^{\prime}<1 / e$. No explosion can occur. If $\delta^{\prime}>1 /$ e the locus cannot intersect $\eta=0$ and explosion will occur. The locus is tangent to $\eta=0$ at $\delta^{\prime}=1 / e, \theta=1$, the critical conditions.

$H_{n_{0}}=\left(A_{1} / A_{2}\right) \exp \left[-\left(E_{1}-E_{2}\right) / R T\right]$ where $A_{1}$ and $E_{1}$ are the preexponential factor and activation energy of the first order reaction forming the catalytic product, respectively. $A_{2}$ and $E_{2}$ apply to the bimolecular reaction of this product with the original material. 
Dubovitskii [59,62] have developed a more detailed and physically more useful interpretation, eq. B-14 and eq. B-15a, using the exponential approximation. They have shown that if $\delta^{\prime}$ is in the range between $1 /\left(\right.$ en ${ }_{0}$ ) and $4 /\left[e\left(1+n_{0}^{2}\right)\right]$ (i.e. $\delta>\delta_{c}^{\prime}$ but is not so large that the initial reaction rate meets the explosion criteria) the system slowly self heats to $\theta=1$ prior to explosion. This slow self-heating can be described to a good approximation by setting $d \theta / d t$ equal to zero in eq. B-15a. Acccording to Merzhanov [14], the equations involving heat conduction in the material for the infinite slab, infinite cylinder, and sphere have been integrated by Barzykin et al [63] for autocatalytic kinetics for various values of the Biot numbers. Ref. [64] also deals with the cylinder, $B i=\infty$ ). The work of Barzykin et al [63] is cited by Merzhanov et al [14] to confirm the fact that eq. B-14 and eq. B-15a may be used with a modification developed by Kuydaev [65] to calculate induction times for these more complex models.

The application of $\mathrm{B}-14$ and $\mathrm{B}-15 \mathrm{a}$ with $\mathrm{B}-22$ to heterogeneous systems (explosives that evolve gases) has been made by Dubovitskii et al. [66, 67] and Merzhanov et al [68]. In ref. [66, 67] the increase in decomposition rate due to a linear decrease in volume in the initial material (due to gas evolution) and the autocatalytic acceleration of a reaction due to the formation of a eutectic ${ }^{+}$of the condensed reaction products with the initial material are examined. In ref [68] an attempt is made to take into account the increase in heat transfer coefficient, ${ }^{\prime}{ }^{\prime}$, due to gas evolution.

\footnotetext{
The phenomena is associated with the idea that the decomposition rate is accelerated by dissolution of the initial material in the product. Rates of decomposition are higher in solutions than solids. Evidently in some cases, if the gaseous products are allowed to escape, no autocatalysis occurs (see [50], for example).
} 
A general method of steady state analysis (i.e. $f(\eta)$ in rate expressions is held constant as in section B.3) has been used to determine critical conditions for chain-thermal explosions, periodic cool flames, and two stage ignitions [69-75]. While this work deals with reactions in gases, the technique is generally applicable to multiple step reactions (the general situation of interest here) though it requires the temperature in the sample be uniform. Moreover, the technique is relatively simple mathematically to apply (see [19], p. 277 and [76]).

Five relatively recent papers have been found that deal with the effect of reactant consumption on the rate expressions for multistep or heterogeneous reactions. Bowes [77] has applied the $\theta, n$ inflection criteria to two independent exothermic reactions, one zero order and the other first order autocatalytic (i.e. eq. B-22). The results seem to have been applied with considerable success [78] to the prediction of ignition of wood sawdust-vegetable oil mixtures for both small samples and large samples. Walker et al [79] claim to have developed an equation to predict ignition temperatures for porous solids undergoing slow heterogeneous (gaseous) oxidation (in accordance with a specified rate law) that apparently corrects for consumption of reactant without going through a non-stationary analysis (i.e. as in section B.5). Two numerical non-stationary analyses dezling with heterogeneous multistep reactions have been located $[80,81]$ but have not been studied. 


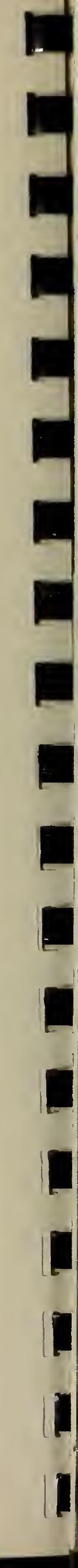




\section{B.8 Thermal Explosions in Linear Heating}

The earlier work on the subject, ref. [82-85] has been reviewed by Merzhanov et al (see [14], p. 285-286). A review by Barzykin [88] includes some later work, ref. [86-87]. An assessment of the results in terms of procedures and assumptions used in thermal analysis has been made by Barzykin et al [89]. These three reviews are the basis for this section.

Linear heating differs from the problems discussed in sections B. 4 . to B.7. in that the uniform ambient temperature, $T_{p}$ increases linearly with time at a constant rate $w$ from some initial value, $\mathrm{T}_{\text {in }}$, where $\mathrm{k}_{\mathrm{T}}$ is negligible. In sections B.4. to B.7., the time dependent problem is for the case where $T_{p}$ is constant and then is step-jumped (i.e. increased at an infinite value of $w$ ) to a constant value $T_{0}$. In both cases, the temperature of the sample is assumed to be uniform and equal to $\mathrm{T}_{\text {in }}$ before the time origin.

The description of the thermal explosion process proceeds in the same manner as that described in section B.I except that now the sequence of explosion temperature experiments with progressively larger values of $T_{0}$ is replaced by a sequence of scanning experiments in which $w$ is progressively increased (i.e. but other parameters are held constant). For very small values of $\mathrm{w}$ it is found that $T-T_{p}$, where $T$ is the mean sample temperature, goes from an initial value of zero to a constant negative value, $\Delta T^{K}$, which does not change with time until $T_{p}$ is near the critical bath temperature, $T_{*}$, in the explosion temperature test. At $T_{p} \lesssim T_{*}, T-T_{p}$ increases, from $\Delta T^{K}$ to a small positive value. After the sample has decomposed, $T-T_{p}$ returns to a negative value. The maximum of $\mathrm{T}-\mathrm{T}_{\mathrm{p}}$ is the order of what is called the Semenov warmup in the step 
jump problem (i.e. $\mathrm{RT}_{0}^{2} / \mathrm{E}$ of eq. $\mathrm{B}-6$ where $\mathrm{T}_{0}$ is now $\mathrm{T}_{*}$ ). It is assumed that the sample container is closed throughout the experiment. For an experiment with $w$ above some critical value, ${ }_{c}$, the value of $T-T_{p}$ rises abruptly near $T_{\star}$ to a maximum that can be much larger than the Semenov warmup. A thermal explosion has occurred. w plays essentially the same role in a scanning experiment that $T_{0}$ does in the explosion temperature test.

There is, however, a basic difference (see [88], p. 32) between development of a thermal explosion in linear and step-jump heating. In step-jump heating, the explosion develops because of an inbalance between heat generation and heat loss. The effect of reactant consumption is merely to add a quantitative correction to the critical condition for this inbalance. In the case of linear heating, thermal explosion occurs when the reactant consumption cannot decrease $f(n)$ at a rate sufficient to compensate for the increase in the rate of heat generation due to the linear increase in the environment temperature ${ }^{+}$. In this sense, heat transfer plays a secondary role.

- An alternative explanation is as follows. If $\mathrm{w}$ is made small, a large amount of material is decomposed prior to the time $T_{p}$ reaches $T_{*}$. Thus, $\delta_{c}^{\prime}$ of eq. $B-48$ is less than $1 /$ e because $f\left(\eta_{c}\right)$ is very much less than its maximum value, $f\left(\eta_{m}\right)$, which is used to calculate $T_{\pi^{*}}$. Thus, no thermal explosion occurs at $\mathrm{T}_{\star}$. However, a thermal explosion, could occur at some higher value dictated by equations $B-47$ and $B-48$. If sample decomposition is always sufficiently large enough so that the calculated thermal explosion temperature exceeds the actual material temperature, then no thermal explosion occurs.

\footnotetext{
Thus, a zero order reaction mechanism always results in a thermal explosion under linear heating.
} 
Numerical solutions in the references reviewed here are for the 1sothermal equations, eqs. B-14 and B-15a, and the unidimensional conduction equations, eqs. B-59 through B-6lc. The reaction mechanisms are for a first order and simple autocatalytic form of $f(\eta)$ (1.e. eqs. $B-21$ for $\mathrm{n}=1$ and $\mathrm{B}-22)$. $\theta$ in eqs. $\mathrm{B}-14, \mathrm{~B}-15 \mathrm{a}$, and $\mathrm{B}-59$ to $\mathrm{B}-61 \mathrm{c}$ is defined in terms of $T_{\star}$ which is the critical value of $T_{0}$ of sections B.4. through B.7. That is

$$
\theta=\frac{E}{\mathrm{RT}_{\star}^{2}}\left(\mathrm{~T}-\mathrm{T}_{\star}\right)
$$

Time is replaced by the variable $\theta_{p}$ defined by

$$
\theta_{p}=\frac{E}{R_{*}{ }^{2}}\left(T_{p}-T_{*}\right) ; T_{p}=T_{1 n}+w t
$$

This yields for eqs. B-14, B-15a, for example

$$
\begin{gathered}
\Omega{\frac{d \theta}{d \theta_{p}}}_{p}=\exp \left(\frac{\theta}{1+\beta_{\star} \theta}\right) f(n)-\frac{1}{\delta}\left(\theta-\theta_{p}\right) \\
\Omega \frac{d \eta}{d \theta_{p}}=\gamma_{\star} \exp \left(\frac{\theta}{1+\beta_{\star} \theta}\right) f(n)
\end{gathered}
$$

where

$$
\begin{aligned}
& \Omega=\frac{C W}{Q A \exp \left(-E / R T_{\star}\right)}=\frac{C W}{Q A \exp \left(-E / R T_{\star}\right)} \\
& B_{*}=\frac{R_{*}}{E} \\
& \delta_{*}^{\prime}=\frac{1+\beta_{\star}}{\operatorname{ef}\left(\eta_{c}\right)}=\frac{Q^{\prime}}{h} \frac{E}{R_{\frac{\hbar}{2}}{ }^{2}} \exp \left(-E / R T_{\star}\right) \\
& \gamma_{*}=\frac{1}{B_{*}}=\frac{R_{*}}{E} \frac{C}{Q}=B_{*} T_{\star} \frac{C}{Q}
\end{aligned}
$$

The same variables appear in eqs. $B-58$ to $B-61 C$ except that $C, Q^{\prime}$, and $\delta_{*}^{\prime}$ are replaced there by $c, Q$, and $\delta_{*^{*}} \delta_{*^{\prime}}$ is given by

$$
\delta_{\star}=\frac{1}{\mathrm{ef}\left(n_{c}\right)} \frac{g_{1}}{g_{2}}\left(1+B_{\star}\right)\left(\frac{B 1}{B 1+g_{1}}\right)=\frac{g \gamma^{2}}{\lambda} A \frac{E}{\mathrm{RT}_{\star}{ }^{2}} \exp \left(\frac{-E}{\mathrm{RT}_{\star}}\right)
$$


Shortly after $T_{p}$ starts increasing, the material goes into what is called a quasistationary state (known in heat transfer literature as the quasi-steady state) in which the rate of change of temperature everywhere in the material is equal to $w$. In this state, the lag of the surface temperature of the material behind $T_{p}, \Delta T_{\text {ext }}$, and the lag of the center temperature (i.e. for the infinite slab, infinite cylinder, and sphere) behind the surface temperature, $\Delta \mathrm{T}_{\text {int }}$, are given by eqs. B-68. These equations apply only when $\mathrm{dh} / \mathrm{dt}$ is negligible.

$$
\begin{array}{r}
\Delta \mathrm{T}_{\text {ext }}=\frac{w C}{\alpha_{o} S}=\frac{w \gamma V}{B 1 K S}=\frac{w r^{2} g_{2}}{B 1 K} \\
\Delta T_{\text {int }}=\frac{w C}{\lambda S /(r / 2)}=\frac{w r V}{2 K S}=\frac{w r^{2} g_{2}}{2 K}
\end{array}
$$

In eqs. $\mathrm{B}-68, \mathrm{~K}$ is the thermal diffusivity. It is evident that since $\Delta \mathrm{T}_{\text {ext }} / \Delta \mathrm{T}_{\text {int }}$ equals $2 / \mathrm{Bi}$ that if $\mathrm{Bi}$ is $20,90 \%$ of the total temperature lag occurs within the material while if $\mathrm{BI}$ is $0.2,90 \%$ of the total temperature lag is between the surface of the material and its surroundings. It is useful to note that $\Delta \mathrm{T}_{\text {int }}$ increases as the square of the characteristic dimension $r$ while $\Delta \mathrm{T}_{\text {ext }}$ increases linearly with $r . \Delta T_{\text {ext }}$ is independent of $\lambda$ or, equivalently, the heat transfer coefficients inside the material. $\Delta \mathrm{T}_{\text {int }}$ is independent of $\alpha_{0}$. Finally, it is useful to note that the time, $\tau_{q s}$, required for the temperature lags to reach $99 \%$ of the values given by eq. B-68 is independent of $w$ and is given by

$$
\tau_{q s}=3 \frac{c}{h^{\top}}=3 C\left[\frac{1}{\alpha_{0} s}+\frac{1}{\lambda S /(r / 2)}\right]=\frac{r^{2}}{K} \frac{3}{2 g_{2}}\left(1+\frac{2}{B i}\right) \quad B-68 c
$$


As soon as heat generation becomes appreciable, the material still remains in a approximately quasistationary state except during a thermal explosion (if it occurs). That is for most of the scanning experiment $d \theta / d \theta_{p}$ is very close to one. Numerical solution of eqs. $B-62$ and $B-63$, or the equations corresponding to eqs. B-58 to B-6lc show that the maximum $\theta-\theta_{p}, \Delta \theta_{\text {max }}$, depends primarily on $\Omega$ and $\gamma_{*}$ and is insensitive to the magnitude of $\mathrm{Bi}^{+}$. Curves of $\Delta \theta_{\max }$ versus $\Omega$ have the same $S$ shape and variation with increasing $\gamma_{*}$ shown by $\theta_{\mathrm{m}}$ vs $\delta^{\prime}$ in fig. 3 of section B. . $^{++}$

Using a criterion for a thermal explosion analogous to that used by Tyler and Wesley [39], discussed in section B.5., one finds that $\Omega$ must exceed a critical value $\Omega_{c}$ that is insensitive to $B i$ and to a first approximation is linearly proportional to $\gamma_{\star^{*}}$. The proportionality constant, $g_{3}$, depends upon the type of mechanism and varies with $\beta_{*}$ (as well $\gamma_{*}$ and $\eta_{0}$ for an autocatalytic reaction). For example, the numerical results for a first order reaction can be represented by

$$
\Omega_{c}=g_{3} \gamma_{*} ; g_{3}=5.23\left[1-2 \beta_{\star}+139\left(\beta_{\star}\right)^{2}\right]\left[1+10 \gamma_{\star}\right]
$$

For $\gamma$ less than $0.01, \mathrm{~g}_{3}$ is constant to within $10 \%$. This value of $\Omega_{c}$ can be predicted within $50 \%$ or better by solving eqs. B-62 and B-63 with $d \theta / d \theta_{p}$ and $\beta_{*}$ set equal to one and zero, respectively. The resulting value of $\eta$ from eq. $B-63$ is put into eq. $B-62$ and the value of $\theta$ that makes $d\left(\theta-\theta_{p}\right) / d \theta_{p}$ a maximum is determined ${ }^{H} \cdot \Omega_{c}$ is determined from the condition that the corresponding value of $\theta_{p}$ be a maximum (i.e. $d \theta_{p} / d \Omega(0)$.

\footnotetext{
tApparently, by contrast $\Omega \mathrm{d} \eta / \mathrm{d} \theta_{\mathrm{p}}$, whether a local or a volume average
value, is sensitive to $\mathrm{Bi}$.

H In other words, thermal explosions become degenerate or difficult to detect when $\gamma_{*}$ is large (e.g. $\gamma_{*} \geq 0.1$ ).

+ At present, we are unable to justify this step adequately on physical or analytical grounds. It gives good semiquanitative results.
} 
Assuming $\Omega_{c}$ is strictly proportional to $\gamma_{*}$, one can derive the form of $w_{C}$ as follows. From eqs. B-64, B-66, and B-68 one has

$$
\Omega=\frac{w C / h^{\prime}}{\delta_{*}^{\prime} R T_{*}{ }^{2} / E}=\frac{1}{\delta^{*}} \frac{\left(\Delta T_{\text {int }}+\Delta T_{\text {ext }}\right)}{{R T_{*}}^{2} / E} \quad B-69
$$

Replacing $\Omega$ by $g_{3} \gamma_{*}$, one has

$$
\mathrm{w}_{\mathrm{c}}=\delta_{\star}^{\prime} \mathrm{g}_{3} \frac{\mathrm{h}^{\prime}}{\mathrm{Q}^{\prime}}\left(\frac{\mathrm{RT}^{2}}{\mathrm{E}}\right)^{2}
$$

To eliminate $T_{*}$, one notes that $\beta_{*}$ varies slowly with $T_{*}$ so:

$$
\begin{gathered}
w_{c}=\delta_{\star}^{\prime} g_{3} \frac{h^{\prime}}{Q}(E / R)^{2}\left(B_{\star}\right)^{4} \\
1 / B_{*}=\ln \left(\frac{Q^{\prime}}{h} \frac{A}{\delta_{*}^{\prime}} \frac{E}{R_{*}{ }^{2}}\right)
\end{gathered}
$$

The dependence of $\mathrm{h}^{\prime}$ on $\mathrm{Bi}$ can be written as

$$
\mathrm{h}^{\prime}=\alpha_{0} \mathrm{~s} /\left[\mathrm{Bi} / \mathrm{g}_{1}+1\right]
$$

Because the material remains to a first approximation in the quasistationary state throughout the scanning experiment if $w \leq w_{c}$, it seems reasonable to propose that the effect of a thin metallic container similar in shape to that of the material will be to alter $w_{c}$ by changing $c$ to the heat capacity of the material plus container and chainging $h$ ' to

$$
h^{\prime}=\alpha_{0} S /\left[B i / 2+\alpha_{0} S / \alpha_{e} S_{e}+1\right]
$$

$\alpha_{e}$ is the external heat transfer coefficient per unit area of a container of total area $\mathrm{S}_{e}$. (Abstracts of the articles indicate that the effect of the sample container has been studied by Grigor'ev et al $[90,91]$ at least in the step jump case of appendices B.1. through B.7.) 


\section{B.9 Experiments on Gaseous Reactions}

The most common experimental method is to admit reactant gases to a thermostatted pyrex sphere fitted with a pressure transducer and containing one (usually movable) or more fine ( $.040 \mathrm{~mm}$ diam.) Pt-PtRh thermocouples. The couples are coated, if required by the chemistry, with silica and arranged to measure temperatures at positions along a diameter of the sphere (to verify thermal explosion theory; at one position to detect the onset of a thermal explosion). Gas at varying pressures is admitted to the vessel at a fixed temperature to determine the critical pressure, $P_{*}$, for explosion. Data are analyzed using eq. $\mathrm{B}-31^{+}$for simple order reactions. Induction times to explosion are viewed as being unsuited to exact analysis [94c]. Precise control to maximize reproduceability of experiments is of key concern. The use of diluents, particularly gases having small heat capacities, to determine the order of reaction from the variation of the critical pressure with thermal conductivity is unreliable. This has been shown $[94 a, c]$ to be due to the compressional heating of the inert gas which can lead to a transient temperature rise above that of the reaction vessel of $200^{\circ} \mathrm{C} !^{++}$Reliable difference calibration of thermocouples can be carried out in place from the temperature change caused by expansion of nitrogen gas (i.e. assuming the expansion is adiabatic and reversibie) [94a]. Thermal conductivities of reactant gases can be calculated, apparently with reasonable accuracy, from thermal diffusivities measured during the warmup of the gas after an adiabatic expansion [95].

troting that $A \sim Z\left(P_{*} / R T_{*}\right)^{n}$, a plot of $\ln \left(P_{*}^{n} / T_{*}^{2+n}\right)$ vs $1 / T_{*}$ has a slope of $-(E / R) n$. Also, $\left(\hat{P}_{*}\right)^{n}$ at constant $T_{*}$ is proportional to $\lambda / r^{2}$.

The dependence on $\lambda$ has problems (see above). The course of the reaction is either followed optically or with the pressure transducer.

Hhe heating effect decreases as the gas heat capacity increases, increases as the pressure increases up to some maximum and then stays constant, and in the case of some gases is accompanied by an initial cooling effect. The transient rise decays in about one second or less for vessels of five cm diameter.

B. 9-1 
The various predictions of thermal explosion theory involved in the method have been verified in detail for simple order reactions when convective heat transfer is excluded. See ref. [94c], in particular. An anomalous critical temperature distribution observed in the early stages of $\mathrm{H}_{2} / \mathrm{Cl}_{2} / \mathrm{NOCl}$ decomposition is attributed to a shift from a heterogeneous to homogenous initiation step in the reaction mechanism [92]. The supercritical temperature distribution corresponding to the ignition regime in methyl nitrate [93] appears to be anomalous, however, since a temperature distribution corresponding to the normal explosion regime is not observed. Raleigh numbers less than 600 are required for convective heat transfer to have a negligible effect on pre-explosion temperature distributions [92].

Recent work in technique development $[96,97]$ has shifted to development of a stirred gas vessel to take advantage of the simplifications in analysis when there is a uniform temperature distribution in the bulk of the gas. The aim of this work is indirect measurement of heat evolution (i.e. product of temperature difference between gas and container times an empirical heat transfer coefficient). 


\section{B.10 Experimental Work on Explosives in the U.S.S.R., to 1970}

The kinetics of decomposition of Tetryl (N-methyl-N, 2,4,6 tetranitroaniline, m.p. 130C) and DINA (dinitroxy diethyl nitramine, m.p. 52.5 C) were determined by measuring heat evolution, weight 1oss, and for DINA also by pressure increase. The experiments were carried out at various constant temperatures in the ranges 130-155 C for Tetryl [98] and 150-170 C for DINA [99]. The mass to volume (available to product gas) ratio, $m / v$, was the same in both methods for Tetryl because of the known variation of the rate constants due to product catalysis ${ }^{+}$(see $[102,103]$ and later discussion). The measurements were made under conditions as nearly isothermal as possible; heat evolution measurements were evidently carried out in a differential calorimeter ${ }^{+}$. The results of the various methods agreed within their combined experimental errors.

The validity of the applicability of thermal explosion theory to DINA [100, 101] was verified by the agreement of the measured and the calculated critical ambient temperatures, $T_{*}$, critical temperature increments and induction times ${ }^{++}$to explosion. Thermocouple measurements of the

The corresponding phenomenon for DINA (see [110]) was evidently unknown then; it is probable that $m / V$ was small for all methods.

+ The reference, identical for both DINA and Tetryl, was unavailable. From ref. [98], the measured temperature difference between thermostat and substance was kept less than $0.5 \mathrm{~K}$ by, apparently, keeping sample weights small enough (e.g. $200 \mathrm{mg}$ ) that heat evolution was less than 23 mW. It seems probable the instrument was not a DTA apparatus since the electrical current strength was altered in steps to give the experimental curve in the form of separate points. This procedure alone would certainly have been insufficient for DTA measurements on DINA.

Induction times to explosion for DINA were calculated by numerical integration. 
temperature onset of explosion were made on 70 to 260 grams of sample contained in cylindrical glass vessels whose outer surfaces were thermostatted by flowing liquid. The samples were kept isothermal by stirring the liquid with a propeller $\left(\mathrm{T}_{*} \sim 150^{\circ} \mathrm{C}\right)$. Measurements were made using five different inner diameters, d, of vessels ranging from 3 to $6 \mathrm{~cm}$ with the sample depths, $l$, equal to or double the diameter. The procedure evidently follows the explosion temperature test described in ref. [8]. The calculated values are for zero order ${ }^{+}$kinetics and the isothermal model parameters are expressed on a unit volume basis. Heat and kinetic ${ }^{+}$data are from ref. [91]. The value of $\alpha^{\prime+}$ was determined by electrical calibration below $T_{*}$ and agrees within $10 \%$ with the value calculated from the thermal conductivity and thickness of the glass vessel wall. Similar agreement was obtained between measurements made on Tetryl [101] using a ring stirrer $\left(T_{*} \sim 150 \mathrm{C}\right)$ and calculations using quasistationary theory with autocatalytic decomposition parameters from ref. [98].

Quanitative methods for studying thermal explosions in small amounts of material, whether they are solid or liquid, should, according to ref. [104], avoid designs that aim at making heat transfer between the material and its environment as large as possible. Small volumes under this condition mean high critical temperatures which can lead to foaming, swelling, etc., that can restrict the applicability of the results. Larger sample

\footnotetext{
Fef. [91] indicates simple first order kinetics. However, the volume of initial reactant decreases as $1-\mu \eta$ where $\mu$ is 0.95 . Thus, in a cylinder, $h^{\prime}$ varies as $1-0.84 \eta$ for $\ell / d$ equal to 2 while $h^{\prime} / V$ varies much more slowly as $(1-0.84 n) /(1-0.95 n)$. Division of $Q^{\prime} \mathrm{d} n / \mathrm{dt}$ by $\mathrm{V}$ yields $\mathrm{Qk}_{\mathrm{T}}(1-n) /$ $(1-\mu \eta)$. Thus, if $\eta$ were 0.1 at explosion, then $h^{\prime}$ and $f(n)$ have decreased by $8.4 \%$ and $10 \%$, respectively, while $h^{\prime} / V$ and $f(\eta) / V$ have increased by $1 \%$ and $0.6 \%$, respectively.

${ }^{+}{ }^{\prime}{ }^{\prime}=h^{\prime} / \mathrm{s}$ or the net heat transfer coefficient per unit area of sample.
} 
volumes reduce the critical temperature but increase errors caused by the increased (generally unknown) temperature gradients in the material. Both critical temperatures and temperature gradients in the sample are reduced by the opposite procedure -reducing heat transfer by introduction of an insulating layer ${ }^{+}$between the sample and a thermostat. Biot numbers of one or less for solids apparently can be achieved for one to five gram samples with cylindrical glass Dewar vessels whose inner diameters (i.e. of the reaction space) are 1 to $2 \mathrm{~cm}$, and outer dianeters (i.e. inner face of thermostat), are twice as large as the inner ( $\alpha \sim 1.3-2.1 \times 10^{-4}$ $\mathrm{WK}^{-1} \mathrm{~cm}^{-2}$ ). Sample depths are of the order of four inner diameters.

Validation of the approach was carried out by demonstrating agreement between measurements and calculations for DINA and Tetryl [104] as in ref. [101] as well as measurements and calculations of a number of induction times under supercritical conditions for Tetryl. Thermocouple temperature difference measurements showed a maximum internal difference in the material of $1 \mathrm{~K}$ for a self-heating increment (i.e. $\mathrm{T}_{c}{ }^{-T_{*}}$ ) of $10 \mathrm{~K}$. $h^{\prime}$ is determined for each sample depth since heat exchange parallel to the vessel walls is not negligible.

A standard size reaction vessel for one gram samples was developed for critical temperature (and induction period) measurements for absolute ranking of thermal instability [104]. Absolute ${ }^{+}$in this context means that $\dot{h}^{\prime}$ of eq. $(B-9)$ is kept at the same known value for all materials

\footnotetext{
This is apparently not common practice in thermography; it corresponds to the analysis of the possible errors in our own experimental work. of necessity, however, the layer must not have a long thermal time constant.

${ }^{+}$Values of $E / R$ can be derived from critical temperature measurements if they are made for different values of $h^{\prime}$-differing $\alpha^{\prime}$ or $S / V$ with due allowance for end effects. From eq. B-9, E/R is the slope of a plot of $\ell_{\eta} T_{*}^{2} h^{\prime} / V$ vs $1 / T_{*}$. QA can be determined from the intercept of the plot if $\delta$ is known (i.e. order, mechanism etc.).
} 
and abnormal swelling or foaming of the material has been eliminated. Consequently, the results can be used as a part of calculations for different conditions. Values of $T_{*}$ and critical induction times for seven explosives are cited and compared with flash point temperature ${ }^{+}$ rankings.

This type of reaction vessel was shown to be suitable for use in single calorimeter scanning (i.e. linear heating or cooling with the thermostat) as well as static (i.e. constant temperature) experiments. This was demonstrated by the agreement between kinetic parameters obtained in the two types of experiment for the decomposition of Tetry1 [106] and the polymerization of styrene [106, 108]. Maximum temperature differences in the materials during scanning experiments were $0.2 \mathrm{~K}$ for Tetryl and $0.3 \mathrm{~K}$ for styrene. The measurement procedure is based on eq. B-3 written in the forms

$$
\begin{gathered}
Q^{\prime} \frac{d \eta}{d t}=c \frac{d T}{d t}+h^{\prime}\left(T-T_{0}\right) \\
Q^{\prime} n=C\left[T(t)-T\left(t_{i}\right)\right]+\int_{i}^{t_{h}} h^{\prime}\left(T-T_{0}\right) d t
\end{gathered}
$$

In these equations, $C$ is the sun of the heat capacity of the material, which varies with $\eta$, and that of the adjacent wall of the reaction container. Besides temperature measurements of $T-T_{0}$ and $T_{0}$, auxiliary measurements are required to determine $C$ and $h{ }^{\prime H}$ and their dependence on $n$ and/or $T$.

This appears to be some variant of the explosion temperature test discussed in ref. [8].

${ }^{H}{ }^{\prime}$ is ordinarily measured as described in ref. [104]. Variations of ${ }^{\prime}$ with $\eta$ and $T$ occur though $S$ and $\alpha$, respectively. In ref. [108], $\alpha \sim T_{0} 0.4$ for a glass Dewar. For cylindrical vessels $h^{\prime} / v$ will be nearly independent of $n$ if the decrease in sample volume, $\mu$, is small (e.g. for Tetryl, $\mu$ is 0.24 ) and, $\ell / d$ is large. If $\mu$ is large, a metal reaction vessel is introduced to make $h$ ' constant and keep the time constant of the "insulator" small throughout the reaction. This procedure works to the extent that heat exchange parallel to (i.e. within) the walls of the Dewar flask is negligible and to the extent that $\alpha_{e_{0}} \ll s / s_{e}$;here $\alpha_{e}$ is the heat transfer coefficient between the thermostat and a unit area of the metal tube having a surface $\mathrm{S}_{\mathrm{e}}$. 
The variation in $C$ with $\eta$ is neglected when its contribution to eq. $B-7$ is small. The variation in $h^{\prime}$ can be eliminated if the reaction vessel wall is made of metal (i.e. large thermal conductivity) rather than glass. Measurements at various scanning rates, w, or constant temperatures are analyzed by setting up plots of $\mathrm{dn} / \mathrm{dt}$ versus $\eta$ for various temperatures ${ }^{+}$.

It is of interest to note that linear heating is recommended for decompositions with large initial rates while a program of linear cooling followed by constant temperature is recommended for strong self-accelerating reactions. Dilution of the sample by an inert solvent, such as alumina powder as a diluent for pyroxylin [112], can be used to increase $\gamma$ (and thus degenerate the thermal explosion) to extend the upper temperature limit of static experiments. The same apparent effect is produced by a sample container of large heat capacity as mentioned in ref. [109]. This does not achieve an identical result since $\gamma$ for a unit volume of sample remains unchanged (unless heat transfer between container and sample is much greater than between container and thermostat).

Apparently an appreciable number of explosives decompose by an autocatalytic mechanism over fairly large ranges of $\eta$ and the degree of autocatalysis increases as the fractions of gaseous products in the reactant is increased. Examples are tetryl [102, 103], pyroxylin [105], and flour [109].

$\bar{t}_{k_{T}}$ is determined from plots of $\mathrm{d} \eta / \mathrm{dt}$ versus $1 / \mathrm{T}$ at constant $\eta$. 
DINA changes kinetics from first order. when no gaseous products remain in the reactant to an autocatalytic type of mechanism when product gases are not allowed to escape [110]. A 50 percent increase in $Q$ and a 100 fold increase in decomposition rate also occur. Measurements [111] of $k_{\mathrm{T}}$ of the DINA decomposition reaction at small $\eta^{+}$, when treated as first order reaction, give values of $A$ and $E$ identical to earlier work [99], apparently when autocatalysis is minimal, and a value of $E$ at pressures above 1 atm identical to that in ref. $[110]^{H}$ for small $n$. Confusing these observations is the fact that an analysis of critical temperature data at $1 \mathrm{~atm}$ in references [110], [104], and [100] by a $\operatorname{lnT}{ }_{*}{ }^{2}{ }^{\prime} / V$ vs $1 / T_{*}$ plot yield an $E$ and $A$ of $35 \mathrm{kcal} \mathrm{mol}^{-1}$ and $2.5 \mathrm{x}$ $10^{13} \mathrm{sec}^{-1}$ instead of $45 \mathrm{kcal} \mathrm{mol} \mathrm{m}^{-1}$ and $3.8 \times 10^{-18} \mathrm{sec}^{-1}$, respectively, used in calculations of $\mathrm{T}_{*}$ in refs. [104] and [100]. One can show that $\mathrm{T}_{*}$ calculated from the first pair of $\mathrm{E}, \mathrm{A}$ is only $3.7 \mathrm{~K}$ larger than that from the second pair. Thus it may be that the only significant problem is the disagreement between ref. [110] and the original data from ref. [99]. An additional complexity in the case of DINA is the decrease in the Semenov warmup as $T_{0}$ is progressively raised above $T_{*}$ when external pressures are 0.4 atm or less! This behaviour and the experimental Semenov plot are consistent with the heat absorption effect of vaporization of reactant in product gas bubbles [113].

\footnotetext{
$t_{B y}$ virtue of $\mu=0.95$, measurements of $T-T_{0}$ times aS/VQ yield $k_{T}$. Apparently $\mathrm{T}-\mathrm{T}_{0}$ were maximal values corresponding to small $\eta$.

Hhe isothermal experiments of ref. [110] were analyzed in terms of first order reaction with $E$ and $A$ varying as a function of $n$.
} 
The experimental work on flour [109] demonstrates the need for studying heat evolution kinetics for various types of mass exchange to establish the gross features of decomposition mechanisms.

The dependence of $\delta_{c}$ on the Rayleigh number, $R a$, needed to account for the effect of free convection in liquids on critical conditions, has been established experimentally for plane parallel and cylindrical geometries [114]. This was done by comparing calculated and measured values of critical temperatures for various known $\mathrm{Ra}$ with $\mathrm{HDI}^{+}$in octyl, heptyl, and benzyl alcohol at large Bi. It is found, for reactions of integral order and these geometries, that $\delta_{c}$ is given by eq. B-77 when Bi is large or equivalently, and that $\delta$ ' in eq. B-3la should be divided by $\phi(\mathrm{Ra})$ after $g_{1} / B i$ is set equal to zero.

$$
\begin{aligned}
& \delta_{c}=\frac{1}{e} \frac{g_{1}}{g_{2}}\left(1+\frac{R T_{o}}{E} \ldots\right) \frac{\phi(R a)}{f\left(n_{c}\right)} \\
& \phi(R a)=1+0.062 \mathrm{Ra}^{1 / 3} ; 10^{5}<R_{a}<10^{8} \\
& f\left(n_{c}\right)=1-2.7(n \gamma)^{2 / 3}
\end{aligned}
$$

The predicted effect of the addition an insulating layer of heat transfer coefficient per unit area, $\alpha_{0}$, outside the material on eq. B-3la would then $\mathrm{be}^{++}$:

$$
\delta^{\prime}=\frac{r^{2} g_{2}}{\lambda g_{1}}\left(\frac{g_{1}}{B i}+\frac{1}{\phi(R a)}\right) A f\left(\eta_{m}\right) \frac{E}{R T_{0}^{2}} \exp \left(\frac{-E}{R T_{0}}\right)
$$

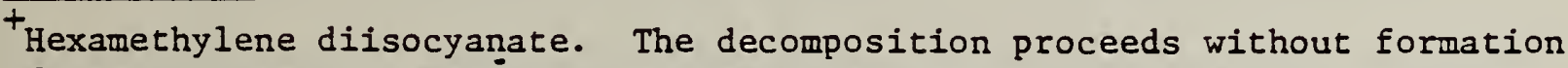
of gas bubbles by a second order mechanism with $E$ and $A$ varying with the solvent; the parameters were determined thermographically. In the formula for $R a, \Delta T$ is set equal to the measured critical Semenov warmup and the viscosity at criticality is the measured value.

HAn alternative form of the terms on the right side preceding $A$ is $\operatorname{Qrg}_{2}\left(\frac{1}{\alpha \phi(R a)}+\frac{1}{\alpha_{0}}\right)$ where $\alpha$ is defined in eq. $B-25$.
} 
The agreement of $T_{*}$ based on the Semenov model with measured values of $\mathrm{T}_{*}$ when $\mathrm{Bi}$ is small (i.e. 2.4-6) but $\mathrm{Ra}$ is large $\left(10^{5}<\mathrm{Ra}<5 \times 10^{5}\right)$ is consistent with eq. B-80 [114]. The applicability of eqs. B-77 to B-80 depends upon the induction time to thermal explosion being much less than a characteristic time required to set up the free convection temperature distribution. The applicability of the equations is also limited to high Rayleigh numbers when decomposition products form gas bubbles [115]. At small values of the Rayleigh number, free convection caused by bubble formation dominates for DINA which results in $\delta_{c}$ passing through a minimum as $\mathrm{Ra}$ increases. 


\section{B.11 Hazard Evaluation Experimental Work (Ref. 116-140)}

Hazard tests are generally classified as sensitivity tests or damage potential tests (see [123], section 2.; [124]; [126]; section 1, [128] par. 2, 17; [129], section $2-2.2)^{+}$. The former assess how easily some external stimulus may provoke an accident or, for self-reaction hazards, initiatedecomposition. The latter assess the consequences or damage potential of the accident.

The applied stimuli or initiating impulse in sensitivity tests can be classified as being mechanical, thermal ${ }^{+}$, or an electric discharge. Mechanical stimuli are impact, friction, and shock. Thermal stimuli can be, for example, different constant ambient temperatures, ambient temperature that increases with time, exposure to a constant radiant heat flux, exposure to flame, or exposure to a hot surface. Associated with each classification of stimuli are the ideas of variations in strength or magnitude of energy input degree to which the energy input is 1ocalized spatially, and the time duration of energy input. Boyars [128], for example, recommends usage of "susceptibility" in place of sensitivity and use of the term "sensitivity tests" to refer to stimuli of short term duration and "stability test" for stimuli of long term duration.

The structure of the content of the cited references of this section is consistent with the explosion research concept that, whatever the type of stimulus, initiation of decomposition is ultimately thermal in

\footnotetext{
The distinction is termed initiation and results of initiation in ref. [129].

Hadiant flux is included because in transport accidents it is thermal
in origin.
} 
origin. (This is explicitly identified in ref. [130], for example, which is concerned with fire and explosion hazard evaluation of materials in general, not just materials designed to function as explosives.) In other words, the result of any initiating impulse is to produce an elevated temperature in a material. The magnitude and spatial distribution of this elevated temperature and the length of time the material is exposed to this temperature distribution would, in principle, be sufficient to characterize the effect of the stimulus [17]. However, the detailed, quantitative mechanisms of the degradation of the energy of the various mechanical initiating stimuli into heat is, in general, unknown.

Damage potential tests assess either directly or indirectly the amount and rate of liberation of energy and gas per unit of mass of decomposing material ([128], paragraph 2) and the possible dependence of these parameters on the total mass of material. They are power tests. Apparently, current practice aims at distinguishing between what are called the three types of chemical explosions. In order of increasing violence, these are usually called homogenous explosions, deflagrations, and detonations. The phenomenology of the three types is discussed clearly in ref. [122]. The distinction betwen the first two types ${ }^{+}$, as described, is less important than between the first two and the last type from a practical hazards point of view.

A homogenous explosion corresponds to the spatially uniform thermal spiral and associated decomposition events when the isothermal model of thermal explosion theory applies to the material throughout the course of

\footnotetext{
The distinction is not artificial in the design of thermal tests. For example dilution thermography aims, by limiting the adiabatic temperature rise, at producing a degenerate homogenous explosion.
} 
the decomposition reaction (i.e. when conditions are supercritical).

This type of explosion would evidently apply, to a first approximation, to exothermic homogeneous decompositions of liquids and gases that are initiated by a uniform elevated ambient temperature and that are present in bulk size (large Rayleigh numbers).

Deflagration is characterized by a distinct reaction zone which propagates through the unreacted material during the course of the decomposition. Propagation is by heat and mass transport; thus, the propagation rate is less than the speed of sound (at most, tens of meter per second). It is evident that this type of explosion can be associated with the thermal wave that ensures under supercritical conditions in the thermal explosion theory model involving heat transfer by conduction when $B i$ and $\gamma$ are large (see section B.2.). It would apply to this model for any value of $B i$ if a supercirtical condition is produced by local heating rather than a uniform ambient temperature (i.e. ignition regime). Deflagration initiated by an elevated uniform ambient temperature would be associated with solids and heterogeneous kinetics, in particular. In practice, hazard tests are primarily oriented towards detecting pressure sensitive deflagrations and conditions, such as degree of confinement, that might cause a transition to detonation or direct initiation of detonation.

Detonation differs from deflagration in that the propagation rate is supersonic $(1-9 \mathrm{~km} / \mathrm{s})$. A shock wave leads the reaction front; the reaction products are gaseous. Detonations can be initiated by the shock or fragments produced by detonating neighbor charges or by spark, friction, etc.

Lack of a quantitative description of the decay of non-thermal stimuli into heat requires that there be a sensitivity test for each stimulus. In transporation accidents, these stimuli are usually considered [116] to be collision, fire, and direct ignition of material spilled from broken containers. From the preceding discussion, the sensitivity tests must assess the ease with which these stimuli can

$$
\text { B. 11-3 }
$$


initiate an explosion, deflagration, or detonation and the power tests must assess the damage potential of these events once they are initiated.

A variety of the mechanical stimulus test in refs [116, 124, and 128] are listed in Table B-4. Although not all of them were designed for testing for transportation hazards, all can be explained in terms of this scheme of accident stimulus and type (i.e., sensitivity or power). The reader is cautioned that the tests 1isted in Table B-4 are examples. Many of them are not included among those permitted in the U.S.A. for testing for transportation hazards. Nor do they match the United Nation Recommendations. Table B-4 shows that this scheme converts an otherwise bewildering array of tests into a more rational assembly. It should be noted that positive results (i.e. material can detonate) in tests 4 to 7 are of significance even if sympathetic detonation is not an issue (i.e. no multiple stores). Positive results mean the possibility of detonation by transition from deflagration must be investigated since this is the more 1ikely course of events in an accident ([122], section 6.2).

Table B-5 lists some of the thermal tests in the cited references of the section in the same way used in table B-4. The 1ist, as is the case with table $\mathrm{B}-4$, is not intended to be complete. It does include, however, many of the thermal tests used in combination with mechanical tests in table B-4 to evaluate the self-reaction hazard of a material. For example, tests 8 through 11, tests analogous to 12 and 13 (see later discussion), and test 16 are used in combination with tests $1,2,5,6$, and 7 by $\operatorname{RARDE}^{+}$ [124] to construct a laboratory scale picture of the hazard of the material. Tests, $8,10,11,14,15$ and a burning test (unlisted) in combination with tests 1, 3, and 4 are recommended by Boyars [128] for evaluating the selfreaction hazard profile of organic peroxides. The results of these tests are organized for purposes of regulation of transport, etc. so as to indicate the degree of hazard of material and both if and how it is to be shipped. One illustration of how such a categorization can be developed is given in ref. [128] (see pp. 26-33 in particular).

\footnotetext{
Royal Armament Research and Development Establishment; the official B.11-4 organization concerned with hazard evaluation. In Great Britain.
} 


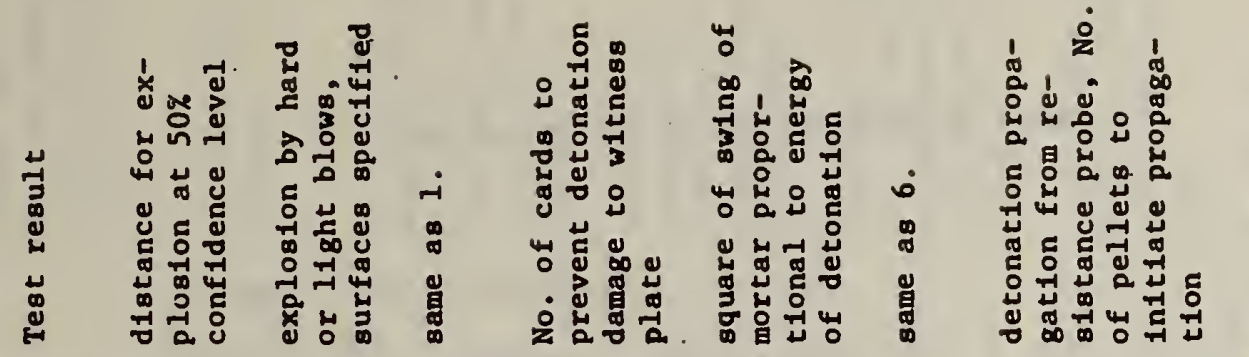

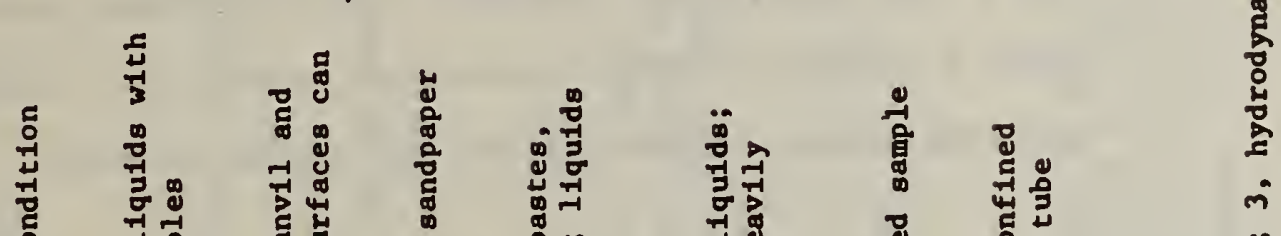

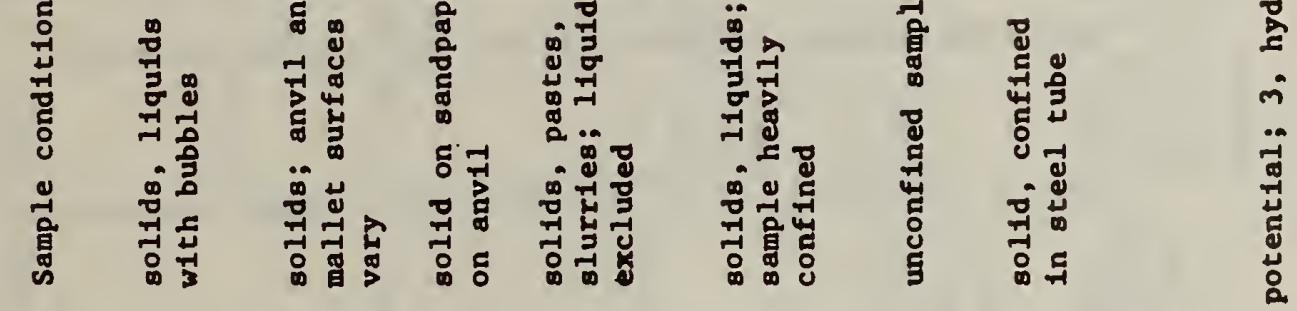

高

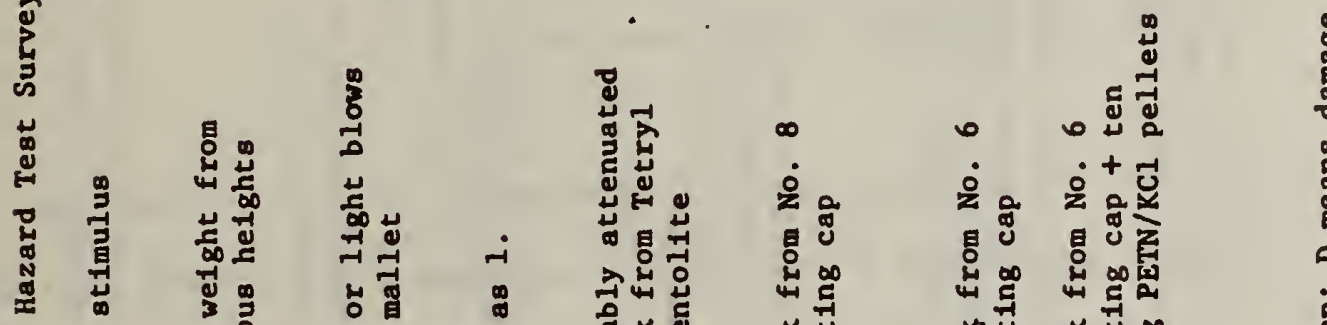

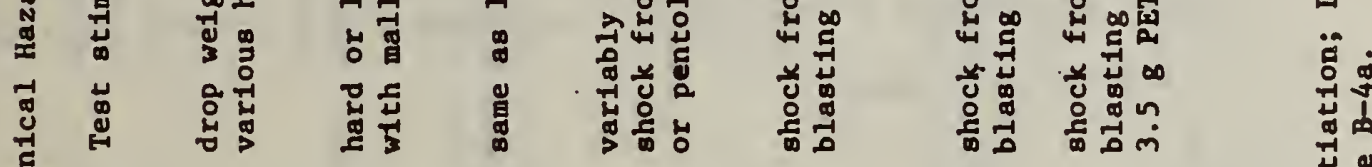

I

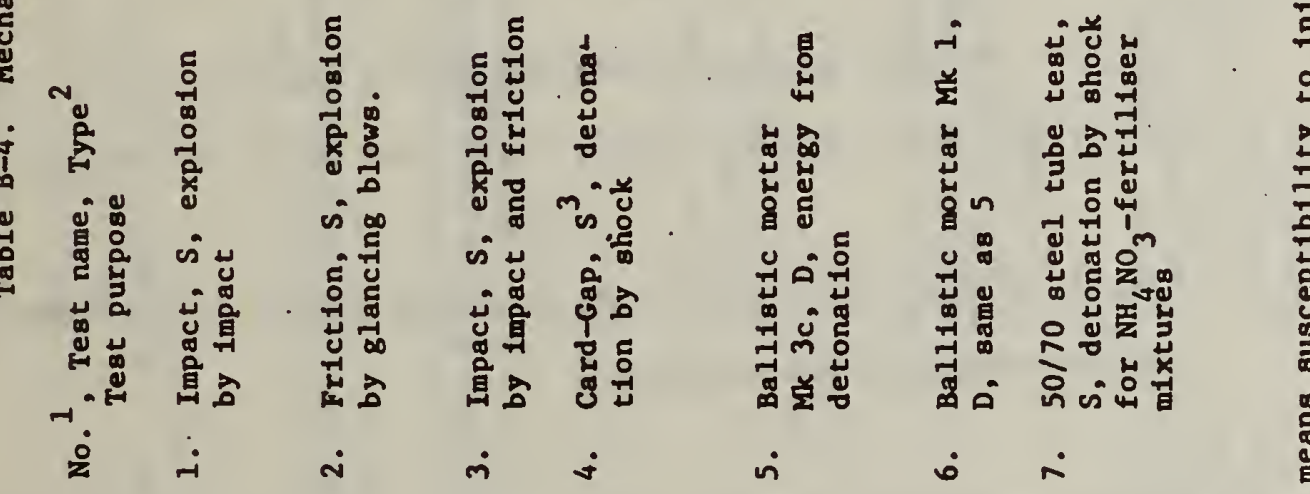

:

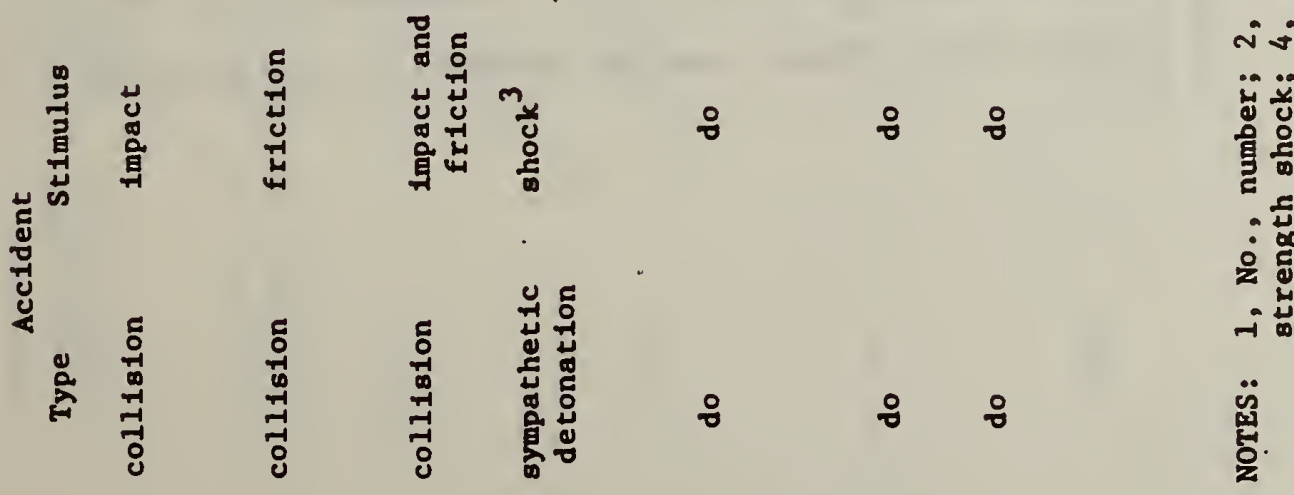


Table B-4a References, Comments, Table B-4

Test No.

References and Comments

1. [8], p. 50, [116]; [123], critique; [124] RARDE description;

[128], p. B5-10, explosion detection by noise; [134], explosion detection by gas evolution; [129], force gauge on anvil.

2. [8], p. 57; [116]; [123] critique; [124] RARDE description.

3. $[8]$, p. $57 ;[123] ;[128]$, par. 48, p. B-3.

4. [8], p. 45; [128], par. 48 indicates D, par. 36,37 indicates

S. As distinct from blasting caps, this test involves only pure hydrodynamic shock.

5. [8], p. 60 , test evaluation misleading-see Appendix A, this report, . and [116] (sect. 6.1.4), [124], [128]; see [123] for critique.

6. [116]; [123]; [124]. This text evaluates effect of containment in determining response to shock.

7. [124]; see [123], section 3, for comments; [122], section 5.4.1 describes a similar test for ammonium nitrate solutions. 


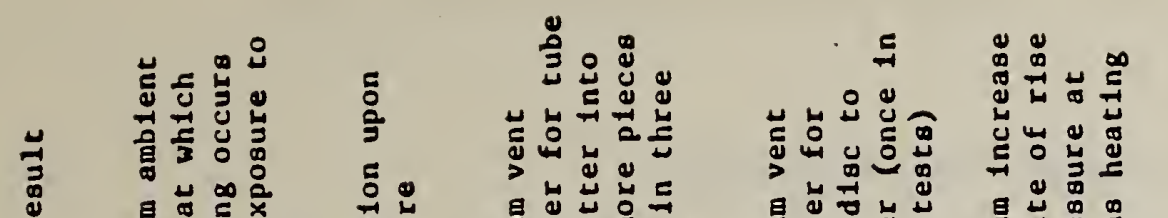

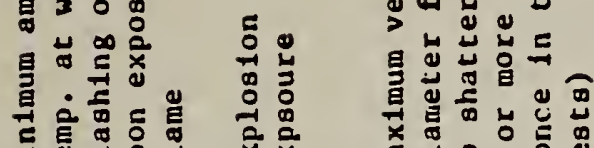

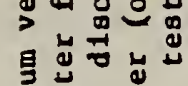

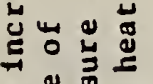

至点

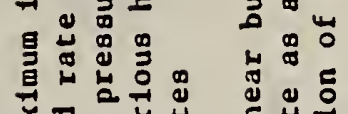

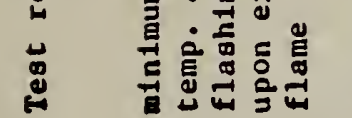

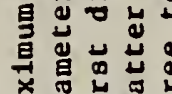

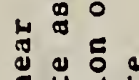

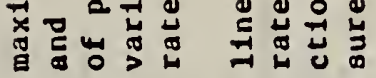

垔

范

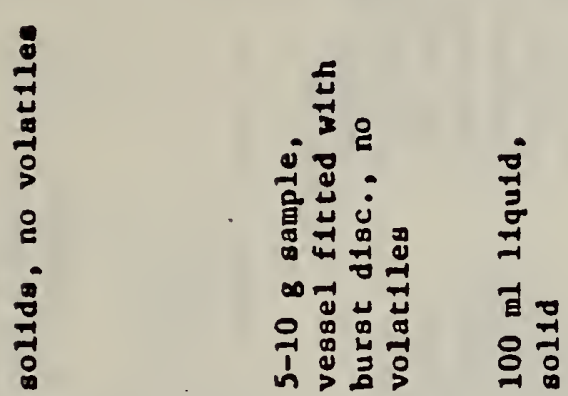

우ㅇㅛㅛ

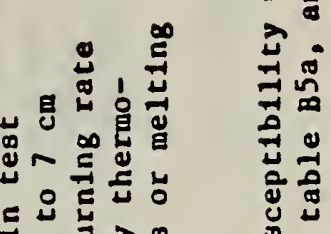

올

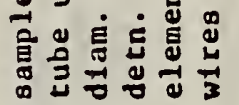

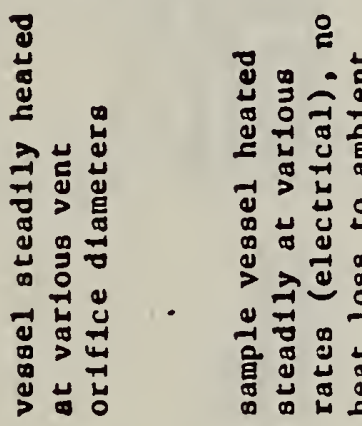

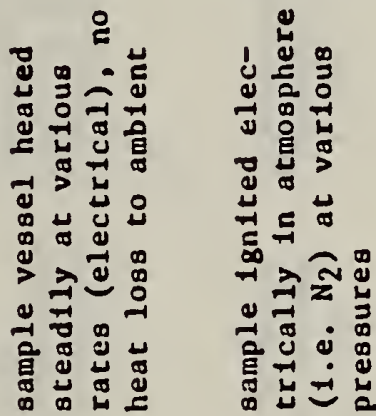

离首

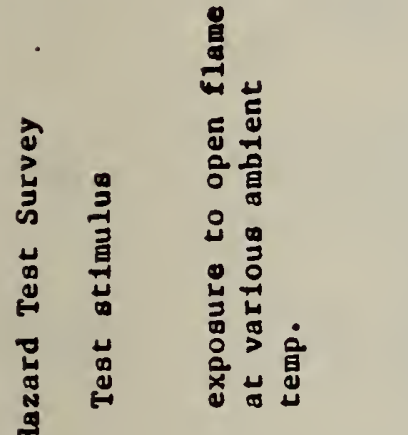

票

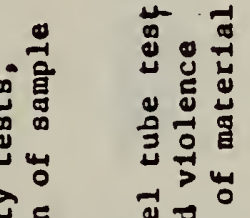

㐘

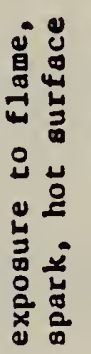

1

$\stackrel{1}{2}$

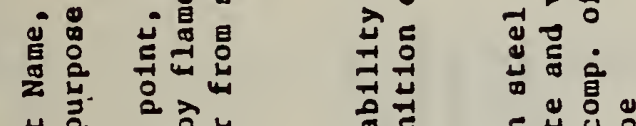

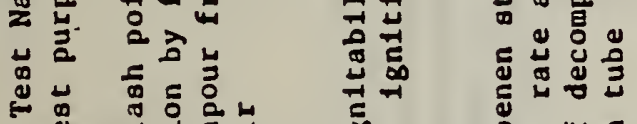

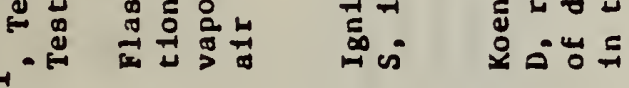

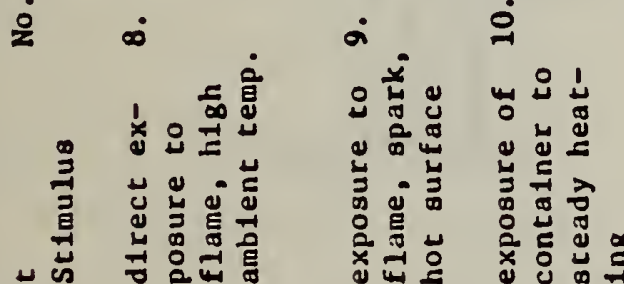

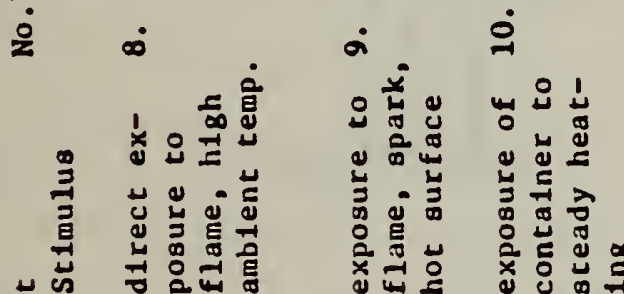

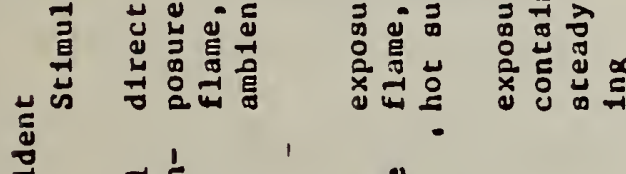

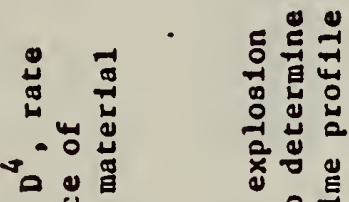

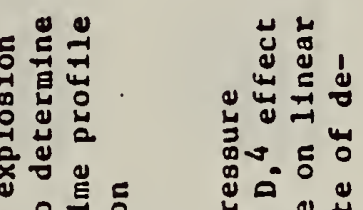

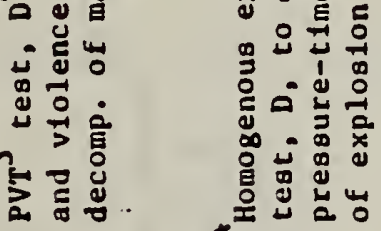

茫荡

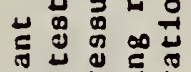

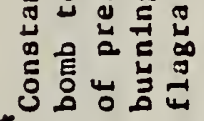

कि

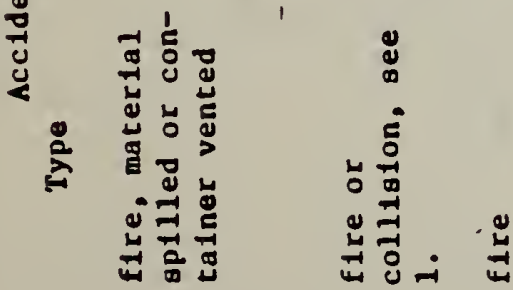

$\stackrel{2}{\approx}$

ت.

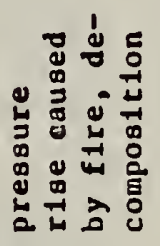

चี

ฐึ

放

总

淟

ปै

岗爵定

飠的

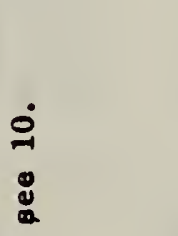

๓

$\rightarrow$

$\stackrel{\circ}{\square}$

* ट्

望莒

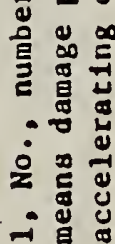

$\ddot{\text { : }}$ 


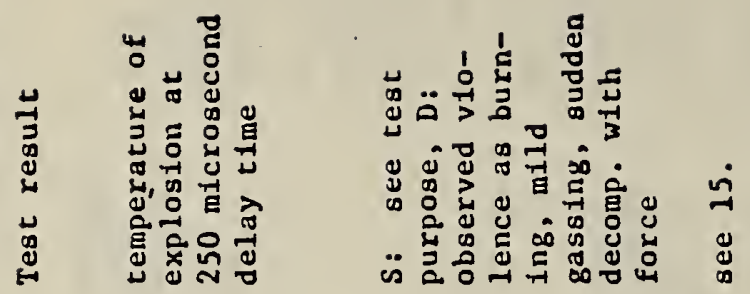

年
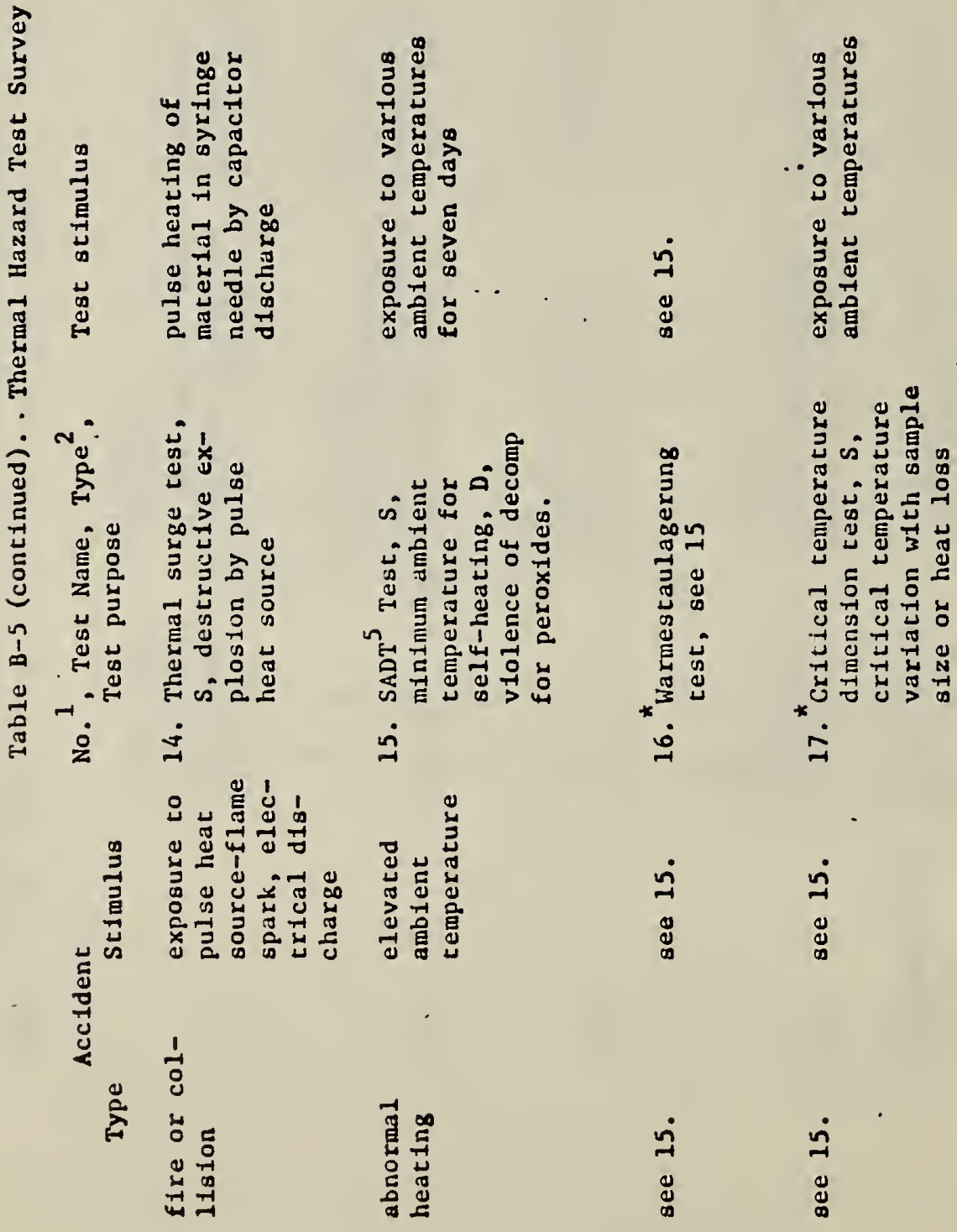
8. [116]; [117]; [124]; [128], par. 21, 23 ASTM Tests D92-66, D93-71, and D1310-67

9. $[116] ;[124] ;[128]$, par. 48 .

10. $[116] ;[124] ;[128]$, par. $17,48[133]$.

11. [116]; [117]; [124]; [128] par. 17, 24, 48; [133].

$\therefore$ 12. [8], p. $35 ;[122]$.

13. [122].

14. [8], p. 25; [128] par. 35, 48, [129], sect. 2-6; [135]; [136].

15. [117]; [128], par. 21, 48.

16. $[116] ;[124]$.

17. [8] p. 37; [119]; [120]; [126]. 
Because of the importance of thermal tests in establishing and developing thermal instability, the following additional commentary on table B-5 seems relevant. Tests 10 and 11 illustrate the attempt to duplicate actual accident conditions in a laboratory test. Evidently, the tests assess the possibility that a violent deflagration or detonation can be set up by confinement and destroy the sample container. In other words, it seems clear that neither of these tests are a simple damage potential test, as Noller et al [117] uses the PVT test. Rather they are a combination of a power and sensitivity test as Boyars (see [128], par. 19) suggests.

Tests 12 and 13 may be viewed as the Dutch (RVO/TNO) version of the second generation of tests 10 and 11 . Tests 12 and 13 attempt to distịnguish between types of chemical explosions, which test 10 or 11 cannot do, and test 13, in particular, attempts to quantify conditions that may lead to violent deflagration and possibly detonation. Tests 12 and 13 have aspects that are characteristic of a third type of hazard test (which can be either a sensitivity test, damage potential test or both) which is called a fundamental parameter test [130]. The distinction is that the physical properties measured in this type of test are used to calculate, for example, sensitivity or power for any hazard situation. It assumes one knows the engineering formula for sensitivity or power. In this particular case, then, the results can be used to evaluate the possibility that decomposition will burst any vessel rather than a specific container as in test 10 or 11 .

\footnotetext{
The U.K. (RARDE) versions of test 12 and 13 seem to be the Time/Pressure [116, 124] and Time/Temperature/Pressure [124] tests. The test stimulus in the Time/Pressure test is ignition of the material via a match head which ref. [124] equates with an accident stimulus of hot spot ignition rather than the equivalent of fire engulfment as in test 12 . However, both the Time/Pressure test and test 12 are damage potential tests, so the difference will be unimportant for many cases.
} 
Test 14, the thermal surge test, can be viewed as a pulse calorimetric (see [137]) version of the Koenen Steel Tube Test with the vent in the latter omitted. Heating of the sample container (syringe tubing with an O.D. and I.D. of the order of $0.36 \mathrm{~mm}$ and $0.18 \mathrm{~mm}$, respectively) by an electric current pulse produces high temperatures in times that are apparently assumed to be short enough to neglect heat loss by the container to its external environment. However, heating of the material is not adiabatic. For large B, the relation between the time to explosion, 'tex' and the container temperature, $T_{0}$, should be approximately that of appendix $B .4$. or the numerical results of Zinn and Mader [33]. This necessarily means that the slope of a plot of $\ln t_{\text {ex }}{ }^{+}$versus $1 / T_{0}$ is not equal to $E / R$. Wenograd, in his original work on the thermal surge test [138], incorrectly states the opposite (i.e. the slope is $E / R$ ) but observes that the values of $E$ so obtained are smaller by half or more than the values of $E$ obtained in other methods. Zinn and Rodgers [139] point out that the relation between $t$ ex and $T_{0}$ in either the thermal surge test or the explosion temperature test (see [8], p. 27 or [140]) is dictated by the condition for rupture of the sample container. Zinn and Rodgers also show that the experimental results of the explosion temperature test (i.e. results of ref. [33] and [139]) and the thermal surge test (i.e. results of ref. [138] on TNT, PETN, and tetryl) are consistent with the relation between $t_{\text {ex }}$ and $T_{0}$ predicted by Zinn and Mader. The values of $\mathrm{E}$ are in reasonable agreement with those obtained by other techniques. Neither Kendall and Rosen [135] nor Janswoude and Pasman [136] mention this second result. Janswoude and Pasman cite (1.e. no derivation given) the following expression for $t_{\mathrm{ex}}$ :

\footnotetext{
tstrictly, in the adiabatic case a plot of $\ln \left(t_{\mathrm{ex}} / \mathrm{T}_{0}^{2}\right)$ versus $1 / \mathrm{T}_{0}$ would
have a slope of $\mathrm{E} / \mathrm{R}$.
} 


$$
t_{e x}=-\tau_{q} \ln \left(1-\tau_{\infty} / \tau_{q}\right)
$$

This result is not consistent with the analysis given in section $B .4$ or the numerical results of $\mathrm{Zinn}$ and Mader and, in our opinion, seems to be in error. Various other possible problems associated with the thermal surge test are discussed by Janswoude and Pasman $^{+}[136]$. It seems evident that the potential. of the thermal surge test to be a fundamental parameter test has yet to be confirmed in the sense of verifying and/or extending Zinn and Rodgers second conclusion.

The SADT test, number 15, (developed.for organic peroxides) detects self-heating by onset of an increase in the temperature of the material (i.e. up to $140 \mathrm{~kg}$ ) in its actual shipping container relative to ambient temperature. As such, the SADT temperature, which may be less than the critical temperature for thermal explosion for the system, has the undesirable attribute that it is directly dependent on the signal to noise ratio of the output of the thermocouple-amplifier detection system. This is not true for a critical temperature test. The SADT test illustrates the following points of general signficance. Since the test is made directly on the material in its container, no scale-up of the results from laboratory sizes is required. The effect of the container is also automatically evaluated. As Clancey [123] points out, the role of the container should, in general, prove to be as decisive for borderline hazardous materials as for explosives. (Test results for the effect on the hazard potential of a material due to a difference in the type of commercial container is given in the applications section of ref. [124] and also in ref. [117], p. 25).

\footnotetext{
${ }_{\text {Effects associated abnormally high critical temperatures of the material }}$ (or model distortions) in this test are mentioned in ref. [136]. See also section B.10. The test was originally deliberately devised to produce high temperatures (see [138]) to examine the hot spot explanation of initiation of explosion by impact.
} 
Elimination and/or evaluation of the scaling problem does not eliminate the other major obstacle in relating laboratory tests to accidents. This bottleneck is the characterization of the mechanical or thermal stresses (and other factors) that may be encountered in an accident. Evidently there is no quantitative description of stress available [123] suitable for transportation conditions.

Test 16 was listed, despite lack of information on details, to serve as link between test 15 and test 17, which can be viewed as a laboratory scale, fundamental parameter version of test 15 . Test 17 illustrates an experimental approach to evaluation of the effect of size on heat evolution kinetics. The test rationale is the extension of the thermal instability test developed by Russian investigators to replace "flash point" measurements discussed in the text and a footnote of section B. 10 .

In test 17 , developed by Bowes, the procedure is to measure the critical ambient temperature, $T_{*}$, as a function of various amounts of material in vessels of different size but the same geometry. The critical temperatures are correlated using eqs. B-30 and B-31 written in the form

$$
\ln \left(\delta_{c} \frac{T_{*}^{2}}{r^{2}}=\frac{-E^{\prime}}{R} \frac{1}{T_{*}}+C_{1} ; C_{1} \sim \ln \left[\frac{Q A O E}{\lambda} \frac{E}{R} E\left(n_{c}\right)\right]\right.
$$

$\delta_{c}$ is calculated for the particular geometry, a circular cylinder of radius $r$ in refs. [119, 120], using measured values of the sample dimensions and heat transfer characteristics of the material in the initial stages of decomposition and of the container. $E$ ' is an apparent activation energy since it will include the $I$ and $T^{*}$ dependence of $\delta_{c}$ through the Rayleigh number (i.e. $\phi(R a)$ of eq. B-77) and mixing of the material by 
gas bubbles (see end of section B.10). $C_{1}$ is a constant which would have the form indicated in the absence of free convection or mixing by gas bubbles. $f\left(n_{c}\right)$ is the correction for reactant consumption prior to explosion. Separate measurements of $\mathrm{E}$ and $\mathrm{A}$, when free convection and the effects of mixing are small, are apparently recomnended to obtain some feel for possible limitations in using the extrapolation equation for other material sizes, geometries, or heat transfer conditions.

There are a number points about Bowes' work on test 17 that deserve clarification because of its importance.

(1) Because measurements were made on benzoyl peroxide, the value of $\mathrm{BI}$, as defined in eq. $\mathrm{B}-3 \mathrm{la}$, is effectively infinite. Only succinic peroxide, of the organic peroxides, (see [117]) shows appreciable decomposition in the solid phase before melting. Under these conditions the dependence of $\delta_{c}$ on Biot number would still be given by eq. B-30 provided Bi is redefined with $\alpha_{0}$ replaced by $\alpha_{e}$, the heat transfer coefficient per unit area of the sample container with respect to the external world ${ }^{+}$. Values of $T_{*}$ comparable to those encountered in real packaging are produced by keeping $\alpha_{e}$ small. This avoids so called "model distortions" mentioned in section B.IO and emphasizes the fact that avoiding these distortions is of practical, as well as experimental importance.

(2) The value of $\delta_{c}$ for a circular cylinder can be evaluated with sufficient accuracy for the purposes of the extrapolation equation by writing eq. B-30 in terms of $\tau_{q}$ defined in eq. B-17 using equations B-23 to $\mathrm{B}-25$ as:

$$
\delta_{c} \sim \frac{k r^{2}}{e \tau_{q}} g_{4}
$$

\footnotetext{
This can be derived by multiplying numerator and denominator of $\delta_{c}$ in the footnote at the end of section B.3. by $\alpha_{e} / \alpha_{0}$ and assuming $\alpha_{0} / \alpha_{e}$ and $\left(t_{c} \lambda g_{1}\right) /$ $\left(r \lambda_{c} g_{c}\right)$ are small in comparison to one.
} 
$K$ is the thermal diffusivity of the material and $g_{4}$ is the correction factor for the error in assuming that $\tau_{q}$ is the reciprocal of the smallest eigenvalue of eq. B-58 with the boundary conditions appropriate to the situation indicated in comment (1). In this case one deduces from heat transfer texts that $k / \tau_{q}$ is given by

$$
r^{2} K / \tau_{q}=\mu^{2}+(\gamma D / L)^{2}
$$

where $\mu$ and $\gamma$ are the smallest values that satisfy

$$
\begin{aligned}
\mu J_{1}(\mu)= & B i^{\prime} J_{0}(\mu) ; B i^{\prime}=\alpha_{e} r / \lambda \\
& \gamma \tan \gamma=B i^{\prime} D / L
\end{aligned}
$$

$D$ is the diameter of the cylinder and $L$ is the height of material in the cylinder and $J_{0}$ is a Bessel function of the first kind. $g_{4}$ can be estimated as the ratio of the known value of $\delta_{c}$ for infinite $B i$ and zero $D / L$ divided by the predicted value of $\delta_{c}$ (i.e. 2.00/2.13). An alternative would be to use eq. B-30 directly with $g_{1} / g_{2}$ replaced by $g_{4}\left[\mu^{2}+(\pi r / L)^{2}\right]$ where $\mu$ is the smallest root of $J_{0}(\mu) . \quad g_{1}$ can be evaluated from $g_{1} / g_{2}$ and $g_{2}$ which equals $[2(1+r / L)]^{-1}$.

(3) In the case of experiments of benzoyl peroxide paste the value of $g_{1} / B i$ was determined directly from thermocouple measurements of the temperature at the center of the sample, $T_{S}$, the container, $T^{\prime}$, and the surrounding constant oven temperature, $T_{0}$, at criticality. Using eq. B25 , one has for the steady state heat balance equation per unit area of container:

$$
\left(\mathrm{T}^{\prime}-\mathrm{T}_{0}\right)=\frac{\mathrm{g}_{1}}{\mathrm{Bi}},\left(\mathrm{T}_{s}-\mathrm{T}^{\prime}\right) ; \mathrm{Bi}{ }^{\prime}=\alpha_{\mathrm{e}} \mathrm{r} / \lambda
$$


In the event that the material did not melt one could still determine $g_{1} / \mathrm{Bi}^{\prime}$ directly by replacing $\mathrm{T}_{s}-\mathrm{T}^{\prime}$ with a measurement of $\mathrm{T}_{s}{ }^{-\mathrm{T}_{s}}$ ' where $\mathrm{T}_{s}$ is the temperature of the surface of the material. $T_{S}{ }^{\prime} T^{\prime}$ divided by $T^{\prime}-T_{0}$ would yield $\alpha_{\mathrm{e}} / \alpha_{\mathrm{o}}$ and $\delta_{\mathrm{c}}$ would be estimated from

$$
\delta_{c} \sim \frac{1}{e} \frac{g_{1}}{g_{2}} \frac{1}{\left[1+\frac{g_{1}}{B 1}\left(1+\alpha_{e} / \alpha_{0}\right)\right]}
$$

This extension of the method would apply to homogenous reactions. It might apply to heterogenoeous reactions but no experimental or theoretical work dealing explicitly with this question has been found.

(4) The induction time to explosion of benzoyl peroxide paste ( $35 \%$ dimethylphthalate) is roughly a factor of 100 shorter than that of dry benzoyl peroxide under essentially indentical conditions of $\delta_{c}$ and $r . T_{*}$ is however, identical (i.e. within $7 \mathrm{~K}$ ). Both the damage potential indicator of the SADT test (see [117]) and Bowes' observations on the nature of the explosion, once initiated, (see [120], p. 293) indicate that the damage potential of the dry peroxide is much greater than for the diluted peroxide. These facts indicate that induction times to explosion are only indirectly related to either susceptibility to thermal explosion, the damage potential of the explosion, or the degree of self-reaction hazard based on a composite of the preceding. Induction times are more relevent to an emergency response system to prevent an explosion by cooling the material once the container has been exposed to ambient temperatures near the critical value. 
(5) The results of Bowes' work in combination with the Russian work [113-115] on mixing by free convection, gas bubbles, and evaporation suggests that Groothuizen et al's comment (see [122], p. 240) that analytical methods are not applicable to these complex situations should be restricted to the qualifications given by the authors. That is, the statement applies to the question of analytical versus numerical calculation of the hazard implications of a fundamental parameter test.

A number of fundamental parameter thermal tests in the cited references for this section are not listed in tables $B-4$ and $B-5$. These are analyzed along with similar types of methods in Appendix $C$. It should be noted in this regard that the autoignition or self-ignition temperature test discussed by Gerlicki [130], Hilado and Clark [131], and Setchkin [132] could be converted into a fundamental parameter test for initiation of combustion of vapours in air. In view of current work on gaseous reactants (see section B.9), it seems probable that results obtained by Bowes' method would be more reliable if stirred reactors of various sizes were used $^{+}$. There are some suggestions (see [116]) that hazards posed by the thermal explosion of vapours from condensed phases with boiling points well above normal ambient temperatures are not important (in comparison to explosions in the condensed phase).

\footnotetext{
tExtrapolation to practical situations involving free convection would then be estimated using eqs. B-77, 78, 79 for the dependence of $\delta_{C}$ on $\mathrm{Ra}$.
} 


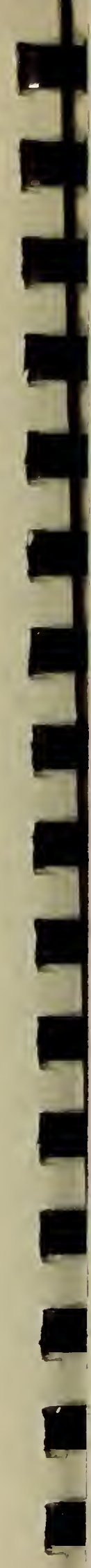

.

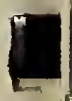

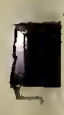




\section{Fundamental Parameter Tests}

\section{C.1. Adiabatic Calorimetry}

Three existing tests, the adiabatic self-heating test developed at NBS (see [8], p. 21 and [142-146]), the adiabatic storage test (see [8], p. 29, [122], [133]), and the ARC test [147] involve adiabatic calorimetry. The first two tests have been evaluated in the second NBS study (see [8]) and the following comments are to correct, where needed; and amplify our first evaluation of these tests.

The purpose of the adiabatic self-heating test is to determine values for $Q A / C$ and $E$, assuming it is found that the Arrhenius form of $k_{T}$ is found to be valid for the initial stages (i.e. $\eta \sim 0$ ) of a decomposition reaction. This test is carried out in an adiabatic calorimeter in which air or another gas is blown past the surfaces of the sample which is suspended below a fan. Fan and sample are mounted in a glass dewar. Depending upon its integrity during the initial stages of decomposition, the sample is suspended unenclosed in a wire basket [144], or in an open beaker [145]. During adiabatic operation, the circulating gas is heated to be equal to the temperature at the center of the specimen. Heat is supplied to a guard cylinder around the dewar to minimize temperature gradients outside the vicinity of the sample. The remaining parts of the test procedure is to determine $C$, the total heat capacity of the sample, by the method of substitution using a glass dewar, stirred water, isoperibol calorimeter. The calorimeter can be calibrated electrically [144, 145] and is operated at room temperature. The thermal conductivity, $\lambda$, of the material is determined for example, by a heat flow meter type of thermal conductivity apparatus (see [144]) that is calibrated with materials of known $\lambda$. Relatively large quantities of material are involved in all 
three tests (i.e. 50-100 gm or more). Thermal instability is evaluated from plots of calculated critical temperatures as a function of the radius of a sphere of the material. $\mathrm{Bi}$ is taken equal to zero.

The adiabatic storage test, in practice, seems to have the same purpose as the adiabatic heating test (i.e. see [122], p. 126, first part of section 5.1.4) and not primarily that given in our first appraisal of the test (1.e. [8], p. 29). As indicated in the latter, the quantity of sample is larger than in the adiabatic self-heating test. Some provision for heat capacity measurement is evidently included (see [122], the ordinate of figure 7 requires one to know heat capacities).

A commercial adiabatic calorimeter, called the ARC, has recently become available that is designed to study exothermic reactions. The instrument is marketed by Columbia Scientific and is based on the design of a unit by D. Townsend, H. Kohlbrand, and coworkers at Dow Chemical [147]. In the commercial instrument [148], samples, nominally of five gram size, are contained in a high pressure vessel. The temperature of the outer surface of the bomb and a surrounding adiabatic shield are measured by thermocouples. Heat is supplied to the shield to keep the two temperatures equal. An additional feature is the ability to monitor the pressure in the vessel.

The main idea behind the adiabatic method is that upon eliminating external heat losses, the temperature of the material undergoing a homogenous exothermic reaction becomes uniform. Thus, from eq. B-58 and the discussion following eq. B-61, one has for a single reaction on a unit volume basis eq. C-I 


$$
c \frac{d T}{d t}=Q \frac{d \eta}{d t}=Q f(n) k_{T}
$$

or for the entire sample eq. C-2

$$
C \frac{d T}{d t}=Q^{\prime} \frac{d n}{d t}=Q^{\prime} f(n) k_{T}
$$

Equation C-2 will apply to a heterogeneous decomposition reaction to the extent that the rate of heat generation is approximately spatially uniform.

If the material, as is the usual case, is placed in a container, neither eq. C-1 or C-2 applies exactly if the adiabatic condition is to minimize heat loss from the container. Heat is supplied to the container by the sample and this introduces temperature gradients in the sample unless it is a well stirred liquid. When the temperature gradient introduced into the material is sma11, one can replace eq. C-2 by eq. B-3 where $T$ in the latter is now interpreted as an average temperature of the material. For the case of heat transfer inside the container solely by conduction, h' can be approximated by eqs. B-23 to B-25. Assuming the temperature of the container and its external environment are uniform and equal to $T_{0}$ and $T_{e}$, respectively, one has for the heat balance equation for the container

$$
C_{o d t} \frac{d T_{0}}{d t}=h^{\prime}\left(T-T_{0}\right)+h_{e}\left(T_{e}-T_{0}\right)
$$

In eq. $C-3 C_{0}$ is the heat capacity of the container and $h_{e}$ is the heat transfer coefficient between the environment and the container. Using eq. $\mathrm{C}-3$ and eq. $\mathrm{B}-3$ one has in place of eq. C-2:

$$
C \frac{d T}{d t}+C_{0} f \frac{d T}{d t}=Q^{\prime} \frac{d \eta}{d t}=Q^{\prime} f(\eta) k_{T}
$$

where $f$ is given by

$$
\begin{array}{llll}
f=0 & , \text { no container, } & T_{e}=T & C-5 a \\
f=1 & , \text { container, } & T_{e}=T_{0} & c-5 b \\
f=1 /\left(1+h_{e} / h^{\prime}\right) & \text {, container, } & T_{e}=T & C-5 c
\end{array}
$$


Equation C-5a applies, for example, to an adiabatic self-heating test where there is either no container or a wire mesh support whose heat capacity is negligible in comparison to that of the sample. In in eq. C$5 c$ is close to one if, as usual, the value of $h_{e} / h^{\prime}$ is much less than one. $C_{0}$ is not necessarily small in comparison to $C$. For example, from the adiabatic storage test results cited in ref. [22] one estimates $C_{0}$ is of the order of $0.25 \mathrm{C}$. Clearly, two heat capacity determinations are required -one for the $C$ of each sample and one for $C_{0}$.

Data analysis is greatly simplified if one can assume that $\mathrm{dT} / \mathrm{dt}$ equals $\mathrm{dr}_{\mathrm{o}} / \mathrm{dt}$ or, equivalently, that material and container are in a slowly varying quasi-steady state. Intuitively, one can see this assumption will hold during the initial stages of the decomposition reaction in terms of time in an adiabatic self-heating experiment. An approximate error analysis confirms this and shows that effect of an error in the asusmption that $\mathrm{dT} / \mathrm{dt}$ equals $\mathrm{dT}_{0} / \mathrm{dt}$ can be expressed as follows. Writing eq. $\mathrm{C}-4$ in the form

where

$$
\mathrm{DT}=\frac{\mathrm{P}}{\mathrm{C}+\mathrm{C}_{0}}(1+\varepsilon)
$$

$$
D=d / d t ; P=Q ' D n
$$

one has for $\varepsilon$ when $C$ is constant

where.

$$
\varepsilon=+\frac{d P}{d T} \frac{\tau_{1}}{\left(C+C_{0}\right)}
$$

$$
\begin{aligned}
& \tau_{1}=\frac{1}{h} \frac{C C_{0}}{\left(C+C_{0}\right)} \\
& h=h^{\prime}, T_{e}=T_{0} \\
& h=h^{\prime}+h_{e}, T_{e}=T
\end{aligned}
$$


This follows from the solution of eqs. $\mathrm{C}-3$ and $\mathrm{C}-4$ which can be expressed, when $C$ is constant, as

$$
\begin{aligned}
D T-D T_{0} & =\int_{0}^{t} \exp \left[(-t+X) / \tau_{1}\right] \frac{d P}{C d X} d X \\
& \sim \frac{\tau_{1}}{C} D P, D P \sim \text { constant } \\
& \sim \frac{\tau_{1}}{C} \frac{P}{\left(C+C_{0}\right)} \frac{d P}{d T}, D T \sim D T T_{0} \text { and } D P \sim \text { constant }
\end{aligned}
$$

$\varepsilon$ in eq. $\mathrm{C}-7 \mathrm{a}$ is proportional to $\mathrm{P}$ since $\mathrm{dP} / \mathrm{dT}$ is given by

$$
\frac{d P}{d T}=P\left[\frac{\left(C+C_{0}\right)}{Q^{\prime}} \frac{d \ln f(n)}{d \eta}+\frac{d \ell n k}{d T} T\right]
$$

One can see from eq. C-8 that when $k_{T}$ is of the Arrhenius form and $f(n)$ is for an reaction of integral order, eq. $B-21$, that $d P / d T$ is small compared to $P$. $\varepsilon$ is small when $P$ is small which occurs during the Initial stages of a decomposition reaction.

In the adiabatic self-heating test, data analysis is carried out by plotting $\ln (d T / d t)$ versus $1 / T$ for the initial stages of the decomposition reaction. The slope is equated to $-E / R$ and the intercept to $\ln \left[Q{ }^{\prime} A f(0) /\right.$ $\left.\left(c+c_{0}\right)\right] . \quad f(0)$ is $f(n)$ at the beginning of the decomposition. It is evident that for this particular type of analysis to be valid, the followirg conditions must be met:

a) only a single reactinn must be occuring

b) $\mathrm{k}_{\mathrm{T}}$ is of the Arrhenius form

c) $h^{\prime}$ must be much larger than $h_{e}$ (i.e. $T_{e}$ equals $T$ )

d) $\left(C+C_{0}\right) \Delta T / Q^{\prime}$, where $\Delta T$ is the temperature range of the plot, must be much lesis than one.

Condition d) is the requirement that both $C$ and $f(n)$ be constant for the data plot. In both the adiabatic self-heating and adiabatic storage tests, the material is not enclosed in a pressure tight vessel so $C$ is 
not necessarily constant. That is, $C$ is of the form

$$
C \sim c_{r}(1-n)+c_{p} n
$$

where $C_{r}$ and $C_{P}$ are the total heat capacity of the initial material and the portion of the products that are condensed, respectively.

In the work of Townsend et al [147], or in the commerical ARC calorimeter mentioned above, the material is enclosed in a pressure container. In this case $C$ is nearly constant because $C_{p}$ in the preceding relation is now the heat capacity of all the products and because now $\left(C_{p}-C_{r}\right) / C_{r}$ is much less than one. In data analysis condition b) is replaced with the assumption that $k_{T}$ has the form $\operatorname{AT}^{2} \exp (-E / R T)$. This makes little difference since $\Delta \mathrm{T}$ is small $^{+}$and has the advantage that eq. C-6a, with $\varepsilon$ set equal to zero, becomes simply integrable. Townsend et al extend the data analysis to larger values of $\Delta T$ when $f(n)$ is not constant by finding a satisfactory fit of the temperature-time data with an assumed form of $f(n)$. $\eta$ is evaluated as $\left(C+C_{0}\right)\left(T-T_{i}\right) / Q^{\prime}$ where $T_{i}$ is the initial temperature in a self-heating experiment when $\eta$ is zero. $\left(C+C_{0}\right) / Q^{\prime}$ can be evaluated as the total temperature rise in the calorimeter caused by complete decomposition of the material.

It is evident that the extension of the data analysis to temperatures where $f(n)$ is not constant is practical only when $C$ is constant. Also it seems clear that the extension requires not only that condition a), given above, be satisfied but also that DT remains nearly equal to $\mathrm{DT}_{0}$ and that the assumptions implicit in the use of eq. B-3 remain satisfied. + It

\footnotetext{
The maximum deviation of a $\ln (d T / d t)$ versus $1 / T$ plot from a straight line occurs near the mean $1 / T$ of the plot, $1 / \bar{T}$, and is of the order of $1 / 4$ $(\Delta \mathrm{T} / \overline{\mathrm{T}})^{2}$ in magnitude.

Hris seems, intuitively, to limit the extension of the analysis to some value of $\eta$ less than that when $D T$ is a maximum.
} 
seems condition a) is, at least in part, a required condition because there is no direct way to test the assumption that $P$ is separable into a product involving $\eta, f(n)$, and $T, k_{T}$. A satisfactory fit of the data is a necessary but not a sufficient condition for this condition be be met. The condition that DT equal $D T$ is desirable both for simplicity and because $h^{\prime}$ will change during the course of the reaction. It should be noted that because the assumption is made that $T$ equals $T_{0}$ in eq. C6a, (as well as DT equals DT ${ }_{0}$ ) in the work of Townsend et al, the value of $\varepsilon$ in eq. C-7a must be replaced by

$$
\varepsilon \sim\left(\frac{C_{0}}{c_{0}+c}\right)^{2} \frac{1}{h} \frac{d P}{d T}
$$

The adiabatic method clearly mimics the hazard situation involved in, for example, loss of cooling of a chemical reactor. It does not, however, exactly mimic the hazard transportation situations mentioned in section B.11 (nor need it do so). 


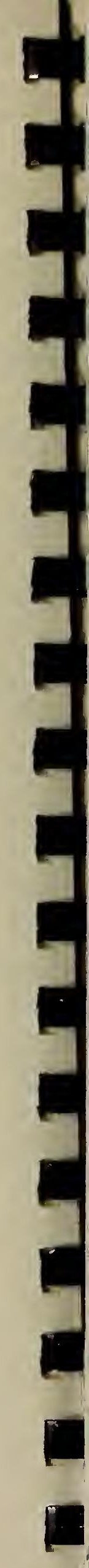

D 


\section{2 Conduction Calorimetry Thermal Instability Tests}

Table C-1 lists some hazard tests that involve heat conduction calorimeters. Tests 1 to 4 involve large sample sizes while test 5 involves (with one exception, the last entry of table C-2) small sample sizes and sensitivities corresponding to commercial DTA apparati that are given in the fourth and fifth columns of table $\mathrm{C}-1$, respectively. The term DTA is used generically in column 1. It is used in a more specific way in column 2 (see entry 5). Nomenclature for DTA, QDTA, and DSC in the second column are based on ref. [154] in the sense that DTA refers to classical DTA, QDTA to Boersema DTA, and DSC to compensated instruments ${ }^{+}$. The definition of the term QDTA given in ref. [154] has not been extended to include twin calorimeters in which a thermopile is interposed between each cell and the common block. The calorimeter in test 1 is essentially that of test 2 but with reference cell omitted. Table C-2 is a sumary of the purposes of the tests listed in table $\mathrm{C}-1$ and can be viewed as supplement to and/or revision (where applicable) to the discussion on pp. $16-20,31-34,39-40$ of ref. [8]. The following comments are made in explanation of the third column of table 7 .

Exact details of the various purposes of tests 1 and 2 are unavailable. The method of data analysis in test 3 seems to be to assume the isothermal model applies and to use eq. B-75 divided by $C$ :

$$
\frac{d T}{d t}+\frac{h^{\prime}}{C}\left(T-T_{0}\right)=\frac{Q^{\prime}}{C} \frac{d \eta}{d t}=\frac{Q^{\prime}}{C} \operatorname{Af}(\eta) \exp (-E / R T)
$$

$h^{\prime} / C$ is determined from observed values of $d T / d t$ and $T-T_{0}$ when $d n / d t$ is negligible. Values of $Q^{\prime} \mathrm{d} n / C d t$ are computed from observed values of $\mathrm{dT} / \mathrm{dt}$ and $\mathrm{T}-\mathrm{T}_{0}$ between the exotherm onset and peak temperatures and

\footnotetext{
This nomenclature is adopted for clarity and is not an endorsement.
} 

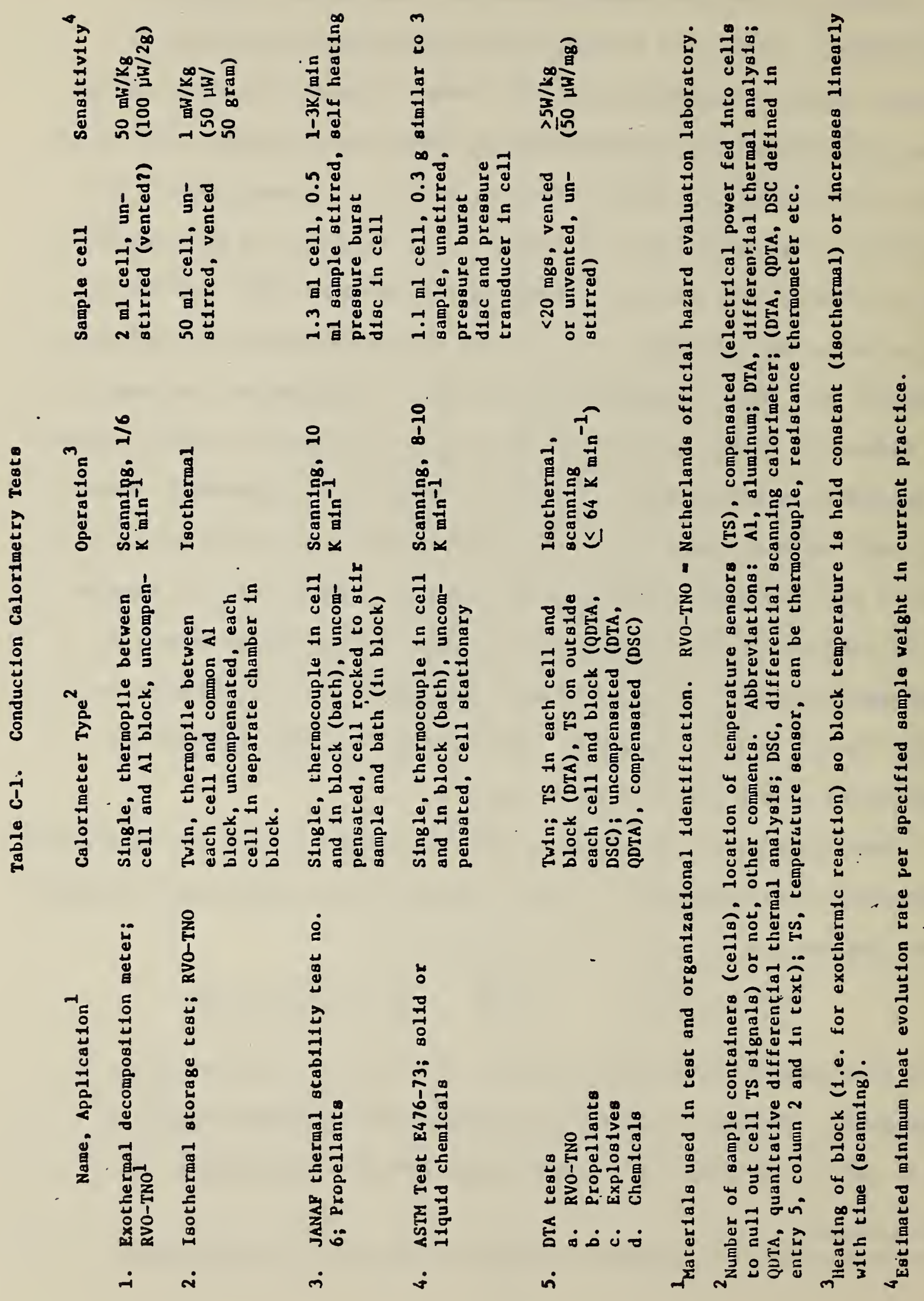

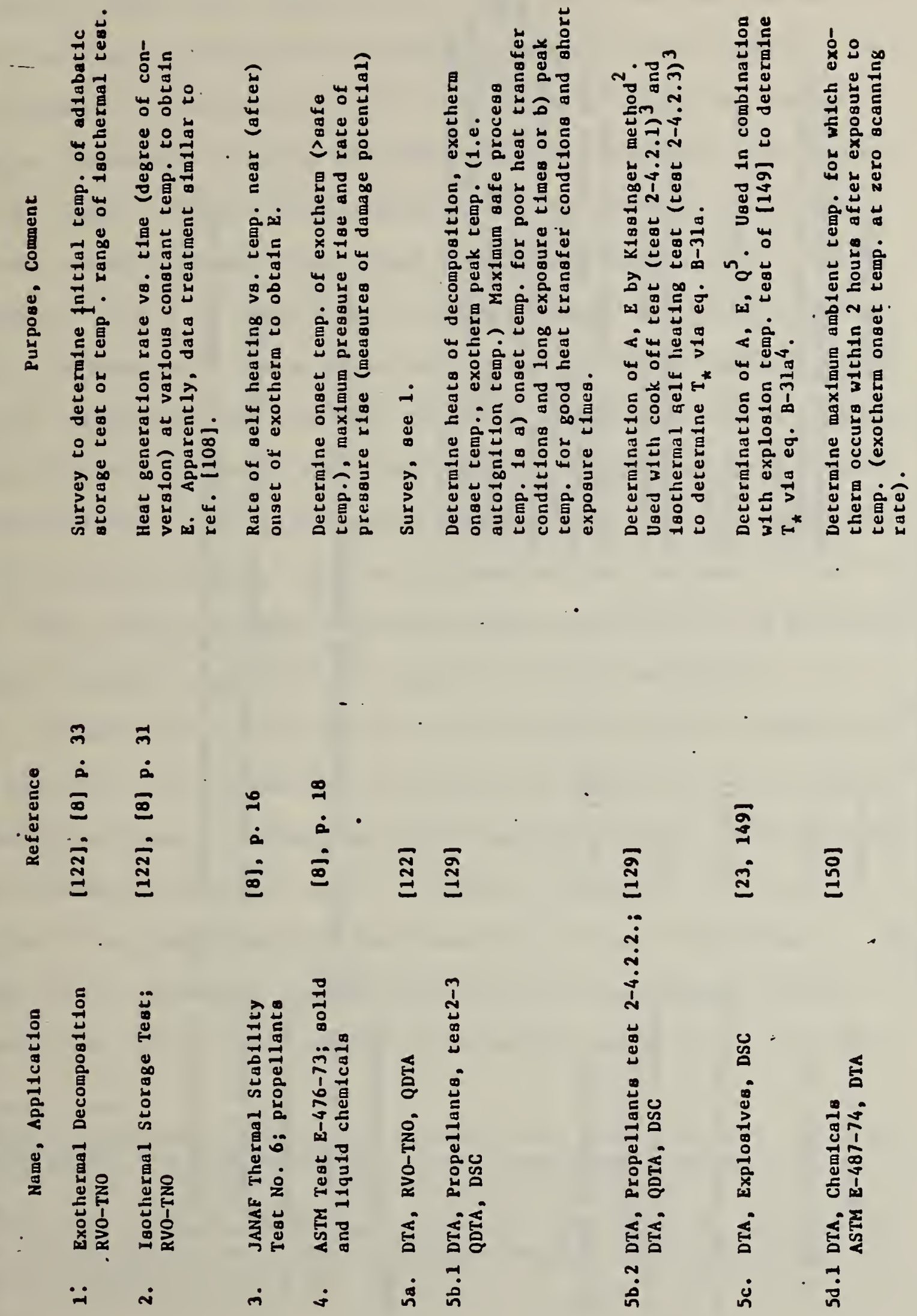


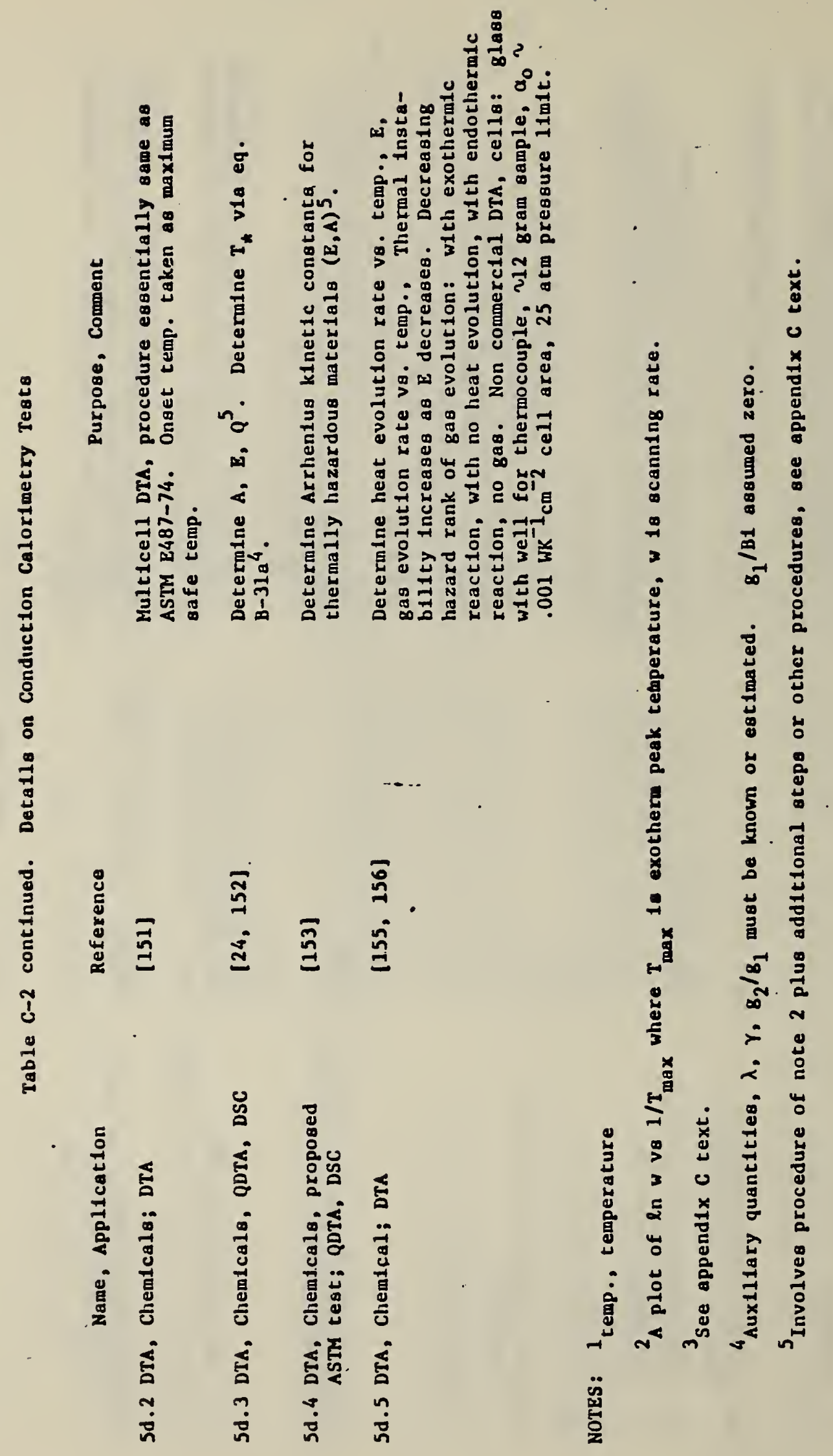


plotted versus $1 / T$. Near the onset temperature (since $\eta \sim 0$ ), the plot should be linear with a slope of $E / R$. While test 4 is based on test 3 it does not seem to incorporate the preceding method of data analysis. This is assumed to be because test 4 is also applied to solids and has no design procedures attempting to insure that the isothermal model applies to the sample. (Test 3 does so although demonstration of the efficacy of the procedure is not given. Test 3 would be relevant only to liquid samp.les.) Test 4 is signfiicant in identifying gas evolution as being part of a measure of damage potential. Also, in specifying that "safe" sample temperatures are less than the onset temperature, test 4 indirectly takes into account that a small sample $(20.3 \mathrm{~g})$ under good heat transfer conditions has a much higher explosion temperature than that appropriate to the material in bulk. This is confirmed by the statement in test 4 to the effect that measurements on small samples would have to be made under nearly adiabatic conditions ${ }^{+}$to obtain $\mathrm{T}_{\star}$ for bulk conditions.

In test 5b.1, the definition of the term auto-ignition temperature ${ }^{+}$ is inferred. The maximum process temperature is stated to be either of those given minus an unspecified safety margin. This is consistent with the preceding comments on test 4. Test $5 \mathrm{~b} .2$ determines the values of $\mathrm{A}$, $E$, and $Q$ from measurements on small samples ( $\leq 20 \mathrm{mg}$ of propellant). "The cook off and isothermal self-heating tests are large sample explosion temperature tests. The former ${ }^{H}$ is evidently similar to the type of

This is consistent with section B.10 and from refs. [88] and [89]. One would expect the peak rather than onset temperature to be near $T_{\star}$.

"The term is used to denote an "explosion" temperature in test 2-5 of ref: [129], and in ref. [150]. The same usage is used in autoignition tests mentioned in App. B.11.

${ }^{+}$Reference containing details was unavailable. 
explosion temperature test used by Bowes (varying propellant sample size under constant heat transfer environment) while part of the latter seems to involve measurement of the Semenov warmup (i.e. under critical conditions $\left.\mathrm{T}-\mathrm{T}_{0} \sim \mathrm{RT}_{0}^{2} / \mathrm{E}\right)$. Test $2-4$ of ref. [129] clearly identifies that there is a scaling problem and that the three parts of the test procedure apply to propellants that decompose by a single reaction and are both homogenous and without voids. (The latter insures that eq. B-3la applies).

Test $5 c$ is significant in that ref. [23] demonstrates that for a number of explosives the measured $T_{*}$ for $40 \mathrm{mg}$ of sample confined in an aluminum blasting cap is in good agreement with that predicted from eq. B-3la (with $g_{1} / B i$ equal to zero ${ }^{+}$) and kinetic constants (E,A) determined

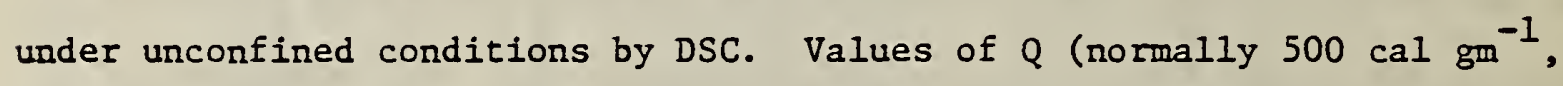

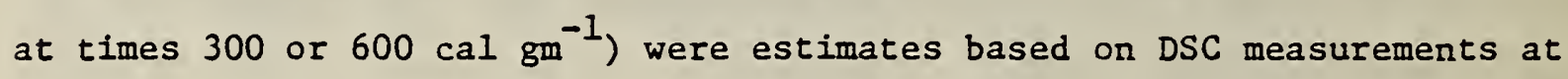
various degrees of confinement. Values of $\lambda$ (between 0.8 and $5 \mathrm{~mW} \mathrm{~cm} \mathrm{~K}^{-1}$ ) were sestimated or taken from the literature. The critical value of $\delta_{c}$ (see eq. B-30) is 0.88 (i.e. the infinite slab approximates this test arrangement). Test $5 c$ is consistent with test $2-4$ of ref. [129] for propellants in recommending that a separate explosion temperature test be made. dowever, test $5 c$ seems to suggest that it will turn out that for many explosives the scaling problem in regards to $\mathrm{T}_{*}$ is not serious ${ }^{+}$. Tests $5 \mathrm{~d} .1$ and $5 \mathrm{~d} .2$ are similar in that both are in effect DTA sample size and heat transfer conditions versions of a time to. explosion and/or explosion temperature test. The ASTM Test E-487-74 correctly

\footnotetext{
With the exception of TATB, the explosives cited in ref. [23] decomposed at their maximum rate in the liquid phase.

\#his would not apply to explosion time measurements. Values of $T_{*}$ are quite high $(200-300 \mathrm{C})$ so it is not unexpected that $E$ and A determined from DSC measurements are consistent with these $T_{\star}$ measurements. However, we are unaware of $T_{*}$ measurements on larger samples or small sample measurements under poor heat transfer conditions that can be compared with predicted values to further clarify the $T_{*}$ scaling problem.
} 
identifies the fact that the value of $T_{*}$, so determined, may not apply to bulk size or heat trasnfer cunditions by stating that $\mathrm{T}_{*}$ is neither the safe storage or processing temperature. The ASTM test uses the values of $T_{*}$ to order materials with respect to their thermal stability.

Tests $5 \mathrm{~d} .3$ and $5 \mathrm{~d} .4$ represent the current status of the state of the art of thermal instability evaluation using comercial small sample apparati. These tests are discussed in detail below and in section $V$.

The last test listed under 5d differs from the other DTA tests in that it involves relatively large sample sizes and happens to be designed so that temperature gradients in the sample due to self-heating are reduced $^{+}$(i.e. since the cell is in poor thermal contact with the block). Identification of gas evolution as an important factor in hazard evaluation is consistent with the aims of test 4 .

An analysis of the third column of table C-2 clearly indicates that a defensible common test purpose for the tests would be to determine those heat evolution kinetic parameters that can predict $T_{*}$ for materials in the bulk under their storage or processing conditions and to measure gas evolution as means to assessing damage potential. Thermal stability is greater as $\mathrm{T}_{\star}$ is larger. Explosion temperature tests under wel1 defined heat transfer conditions can serve as an independent check of the heat evolution measurements if these conditions are the same as the latter. They can be used to evaluate scaling problems if conditions are closer to those in bulk.

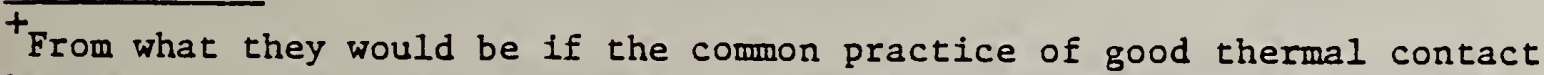
between block and cell were used. The particular design was adopted in an effort to increase the DTA signal.
} 


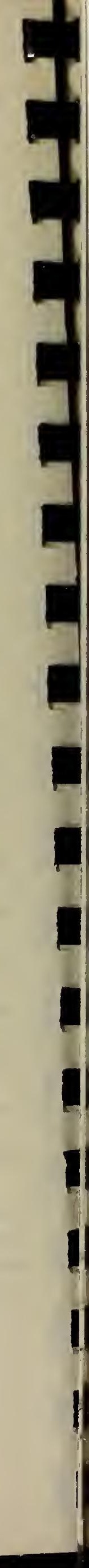


C.3. Simplified Measurement Theory of Quantitative Differential Analysis (QDTA) and Differential Scanning Calorimetry (DSC)

The nomenclature in regards to DTA, QDTA, and DSC is that specified for colum 2 of table C-1 in Appendix C.2. Only QDTA and DSC types of apparati are discussed and of these one specific example of each: the QDTA apparatus described by Baxter [173] and the comercial DSC apparatus used in our experimental work, to be referred to as the DSC-2, that is . described in refs. [154, 174-178]. This presentation supplements the comparison of the operation of the two types of instruments worked out by Gray [179]. It extends the cumparison to provide a complete set of equations for analyzing the experimental data. In particular, our purpose here is to show how $d \eta / d t$ and $\eta$ are determined from the measured output signals, assuming only a single decomposition reaction is taking place. The QDTA measurement equations are summarized first in Sections C.3.1. and C.3.2. The corresponding DSC equations are discussed in section C.3.3. Calibration of the energy and temperature scales are sumarized in sections C.3.4. and C.3.5.

\section{C.3.1. Measurement Equations for QDTA}

A simplified cross sectional diagram of the QDTA apparatus to be analyzed is given in figure $\mathrm{C}-1$. The hollow cylindrical block, whose temperature can be programmed to be constant, increase, or decrase, is in good thermal contact with the constantan sheet, CS. The chromel wires, $\mathrm{Cr}$, are attached directly to the sheet and, form a difference couple with the constantan for determining the difference in temperature, $T_{s}$, of the area of the sheet immediately below the sample container, 1 , and the temperature, $T_{r}$, of the area of the sheet beneath the reference 


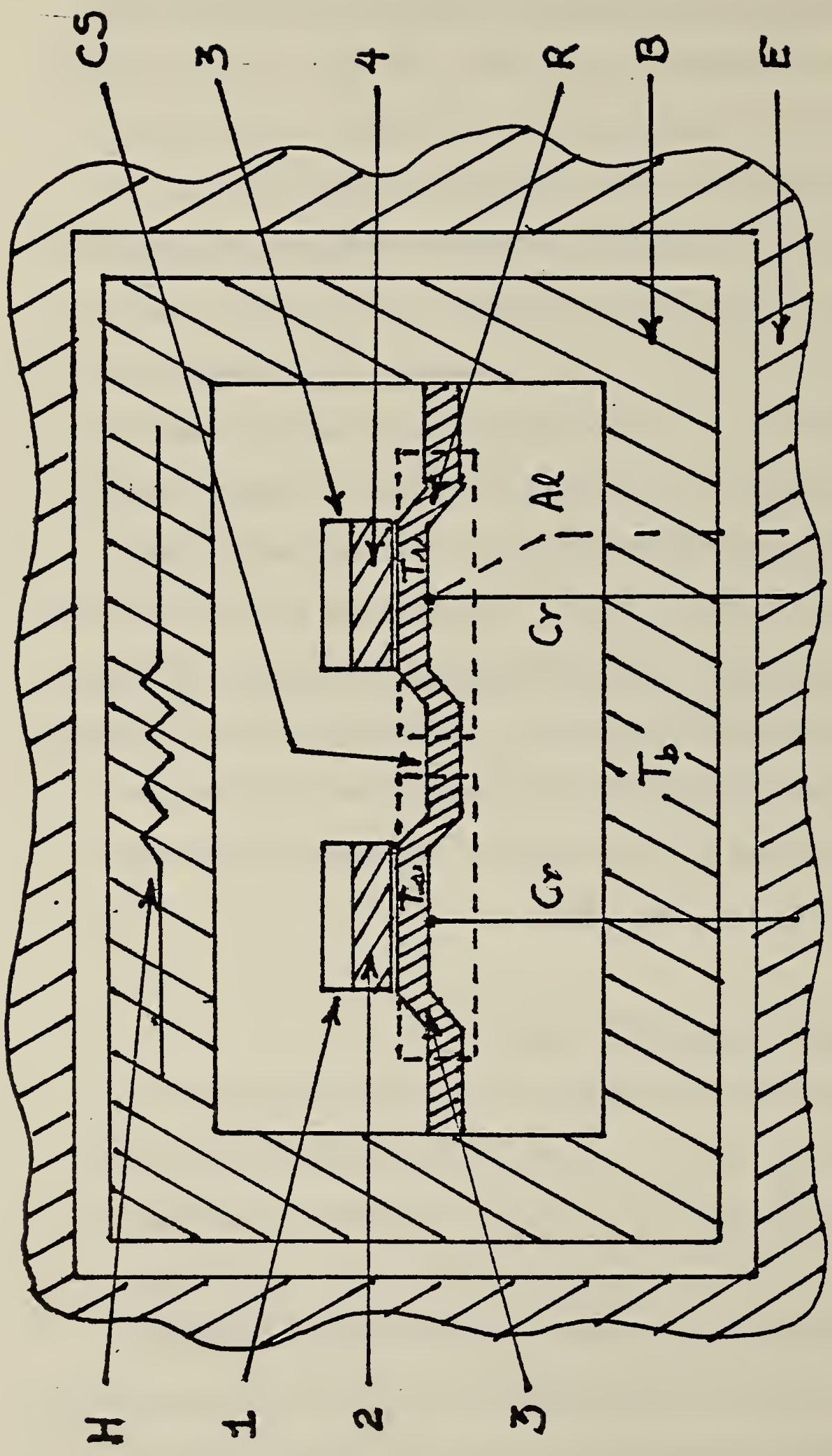

$\stackrel{4}{-1}$

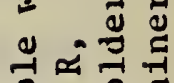

둘..오 오

뇽

글

द्र

\&

I1 4

-

山象

○ 눙

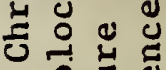

4 D

-

... त् म्न

ก 글

ก

I 일 ....

y है

d)

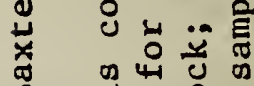

( )

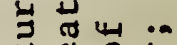

ปี

‡

ㄷำ

बढ़्ठ

山 4 잉

कै Uै है

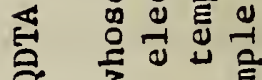

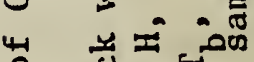

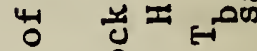

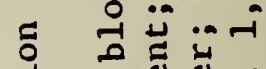

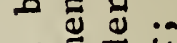

๑

... 낙윽

ข

니로

उ) है

() ह ह

न्मू का हत

फु

㐘 凹

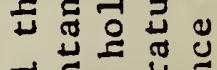

का ซี क म

芶苟魚㫕

त्र 0 ป

रंध 
material container, 3. The single alumel-chromel pair forms one junction of the two junctions of a thermocouple to determine the absolute value of $T_{r}$. The symbols $S$ and $R$ of the diagram refer to portions of the constantan sheet that.are assumed to have uniform temperature, $T_{S}$ and $T_{r}$, respectively, for the purpose of a simple lumped constant or finite element analysis (i.e. see $0^{\prime} \mathrm{Neill}$ [176] or David [180]). The corresponding electrical diagram for this analysis is given in figure $\mathrm{C}-2$. The node potentials are temperatures, capacitors are heat capacities, and the ideal current source of magnitude $P_{2}$ is the total rate of evolution of heat by the material. The ideal voltage source of potential $\mathrm{T}_{\mathrm{b}}$ is the temperature of the block. The conductance from nodes $j$ to $i$ is a heat transfer coefficient $h_{i j}$ and figure 15 assumes that $h_{i j}=h_{j i}+$. Sample and reference materials, their containers, and sample and reference holders are all assumed to have uniform but different temperatures. In effect, we automatically assume there is no calorimetric error due to non-equivalent sources (i.e. see [181]).

The energy balance equations corresponding to figure $\mathrm{C}-2$ are summarized in table C-3. D is the ordinary differential operator in time, $d / d t$, and the remaining symbols and subscripts for entries 1 through 5 of table $\mathrm{C}-3$ are defined in the key to figure C-2. Entries (i.e. row) I; 2, and 3 are the energy balance equations for sample, sample container; and sample holder, respectively. Entry 4 is the sum of entriés 1 through 3 (and assumes heat transfer coefficients are symetric). Entry 5 corresponds to entry 4 for the reference material, container and holder. Entry 6 is entry 5 minus entry 4. Entry 6 contains the approximation

\footnotetext{
This will not be the case where convective transport is concerned unless turbulene dominates laminar flow in the gas flusing the outside of the sample and reference containers and in the gaseous products of decomposition of the sample.
} 

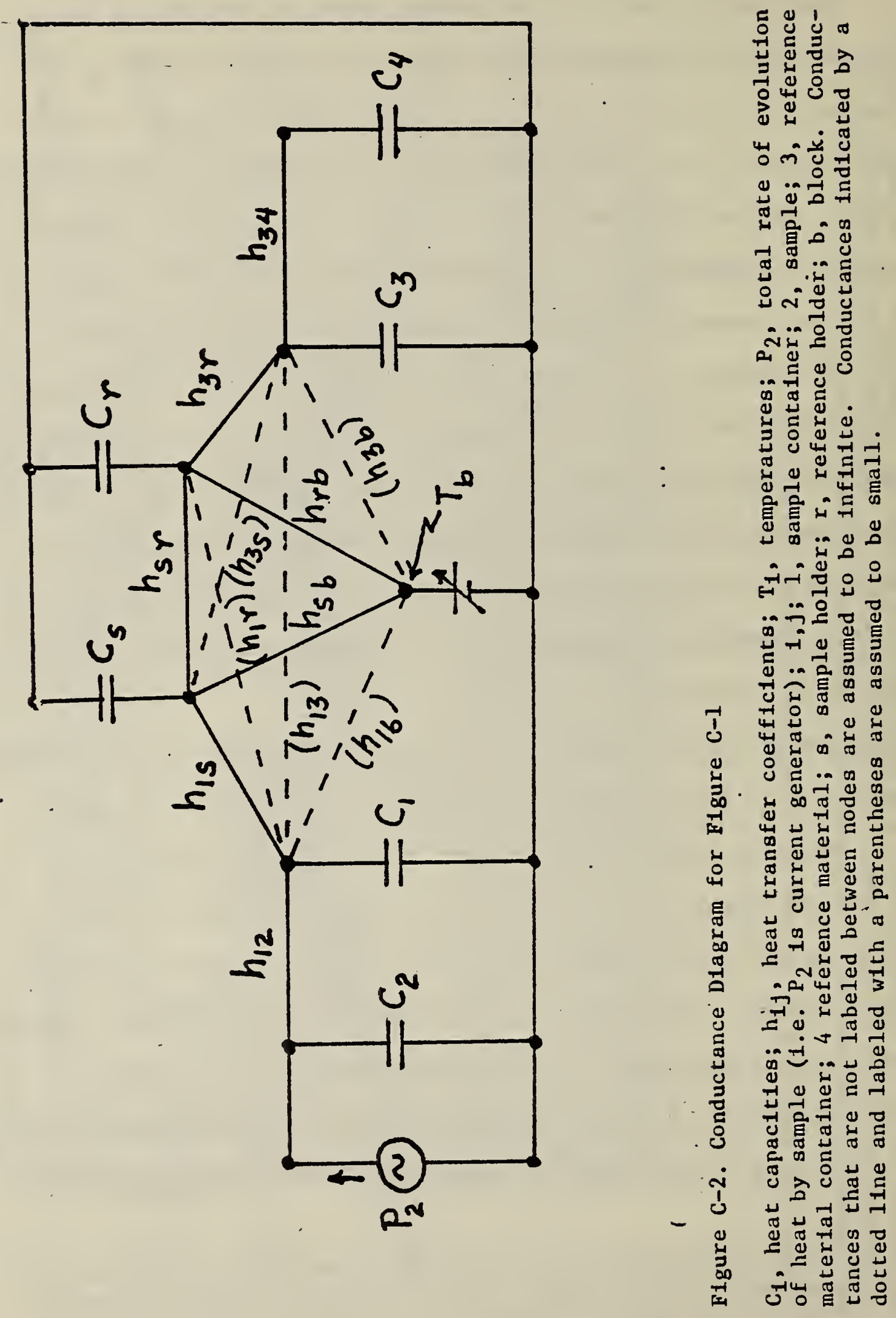


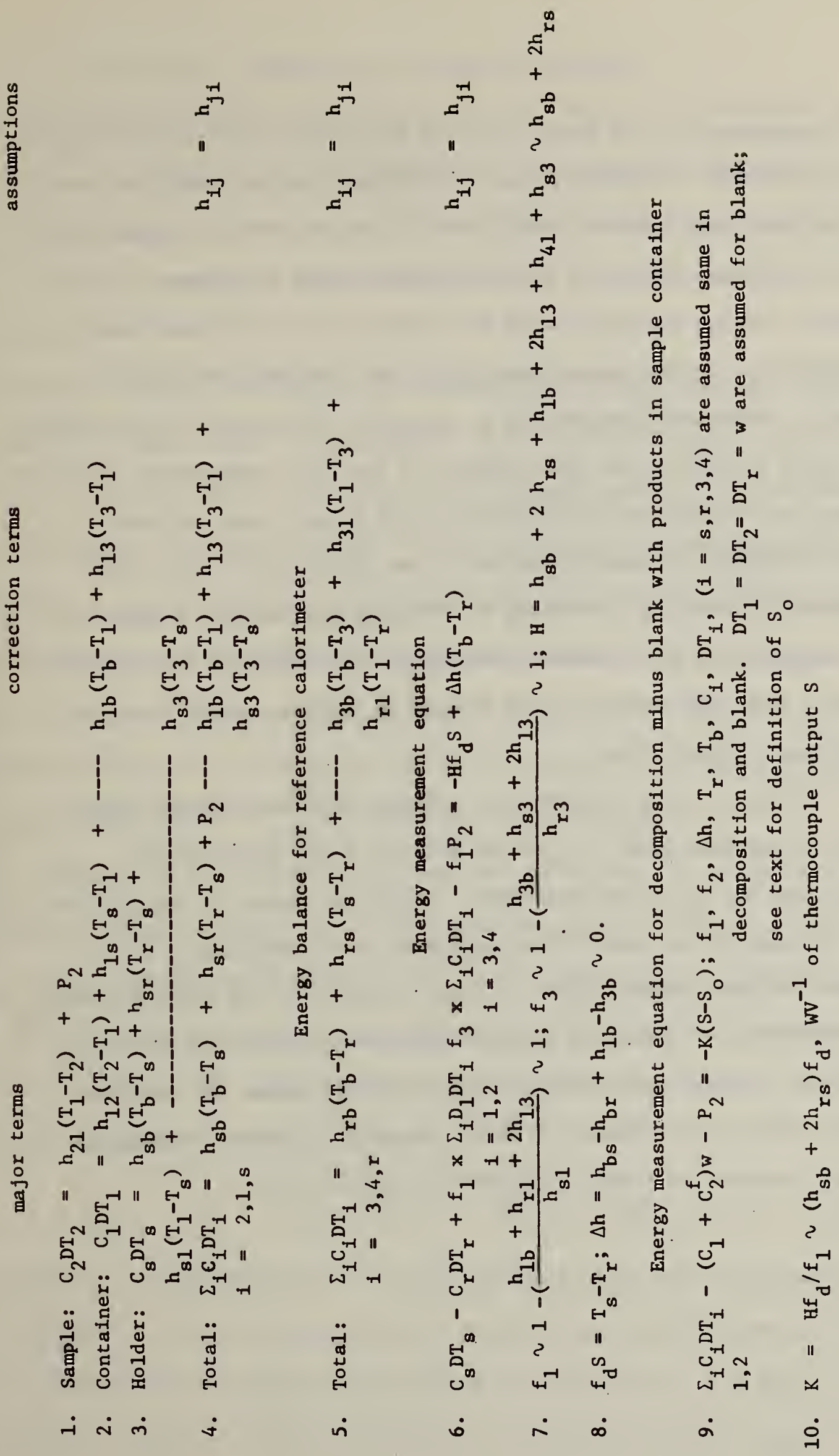


that temperatures in the terms involving small heat transfer coefficients can be estimated by values derived from the heat balance equations when all the small heat transfer coefficients of figure $\mathrm{C}-2$ are assumed to be zero. The values of $\mathrm{f}_{1}, \mathrm{f}_{3}, \mathrm{f}_{\mathrm{d}}, \mathrm{H}$, and $\Delta \mathrm{h}$ of entry 6 are given in entries 7 and 8 . The first value for each is for the aforementioned approximation and the second value is for the case where small heat transfer coefficients are assumed to be zero at the outset of the analysis.

Entry 6 neglects the time constant of the block temperature regulation system (i.e. detector-electronics-electrical heaters plus the heat balance equation for heaters and block) and thus will not apply until transients in starting a scanning or step jump program have decayed out. In a sprogram, $\mathrm{T}_{\mathrm{b}}$ is increased or decreased with time at a constant rate w. In a step jump program, $\mathrm{T}_{\mathrm{b}}$ is changed as rapidly as possible to a new lower or higher value.

$S$ or $\left(T_{s}-T_{r}\right) / f_{d}$ of entry 6 is the instantaneous ordinate output signal of the instrument. $f_{d}$ is the product of the thermoelectric coefficient of the chromel-constantan difference couple of fig. C-1 in $\mathrm{K} \cdot \mathrm{V}^{-1}$ divided by the gain of the difference signal amplifier. The observed ordinate output signal, 0 , lags $S$ because of the thermal inertia of the difference couple and the finite response time of the difference amplifier. In the next section. C.3.2., we shall assume the relation between $S$ and 0 , the ordinate transfer function, is known. Discussion of this transfer function is postponed to section C.3.4. 


\section{C.3.2. QDTA and DSC Equations for Kinetic Anaiysis}

The energy measurement equation for our DSC, which is discussed in section C.3.3., is similar in form to entry 6 of table C-3. Also the temperature equations are similar in form to those to be derived below. Thus the following summary of determining kinetic parameters applies to both instruments.

In kinetics one is interested in obtaining $P_{2}$, since this is equal to Qdn/dt, and the temperature of the sample, $T_{2}$. To obtain $P_{2}$, it is first necessary to subtract out the effects of the sample holder and the reference calorimeter and its contents. It is convenient to generate the blank signal, $S_{o}$, by rerunning the temperature program for $\mathrm{T}_{b}$ used in the decomposition run with everything the same except now only the sample products, having a heat capacity $\mathrm{C}_{2}{ }^{f}$, are in the sample container. The measurement equation for this blank run will be entry 6 of table C-3 with $C_{2}, S$, and $P_{2}$ replaced by $C_{2}{ }^{f}, S_{0}$, and zero, respectively.

The products of the reaction are assumed to undergo no transition or phase change during the blank run. Also, we assume $D T_{S}=D T_{T}=w$ and that both $T_{b}$ and $T_{I}$ increase with time as wt. This automatically requires $D T_{i}$ $=w$ for $i=1,2,3,4$ so long as the heat capacities and heat transfer coefficients of fig. C-2 change slowiy with temperature. Hence, the measurement equation for the blank run is

$$
\left[C_{s}-C_{I}+f_{1}\left(C_{1}+C_{2}^{f}\right)-f_{3}\left(C_{3}+C_{4}\right)\right] w=-H f_{d} S_{0}+\Delta h\left(T_{b}-T_{L}\right) \quad C-10
$$

Subtracting eq. C-10 from entry 6 and rearranging with the assumptions that $f_{1}, f_{3}, \Delta h, T_{r}, T_{b}, D T_{i}$, and $C_{i}(i=s, r, 3,4)$ are the same for both blank and decomposition runs yields entry 9 of table $\mathrm{C}-3 . \mathrm{K}=\mathrm{Hf} \mathrm{d}_{\mathrm{d}} / \mathrm{f}_{1}$ is the calibration constant of the instrument and will vary with temperature. 
The second step is to evaluate $\mathrm{C}_{1} \mathrm{DT}_{1}+\mathrm{CDT}_{2}$. Three approaches are presented in Table C-4. Following Brennan et al [182], the Method 非 is to assume $T_{1}=T_{2}=T_{s}$. This method assumes the sample, sample container, and sample holder are in perfect thermal contact. $\mathrm{C}_{2}$ is assumed to vary linearly with $n$ as given by entry lb (i.e. row b of column $\#_{1} 1$ ) of table $C-4$. $\mathrm{C}_{2}^{i}$ is the heat capacity of the sample before the decomposition starts and $\mathrm{C}_{2}^{f}$ is the heat capacity of the products left in the sample container after the decomposition has ceased.

- Entry $1 c$ is derived by inserting entries $1 \mathrm{a}$ and $1 \mathrm{~b}$ of table $\mathrm{C}-4$ into entry 9 of table $\mathrm{C}-3$ to obtain

$$
\left(c_{2}^{i}-c_{2}^{f}\right)(1-\eta) w-P_{2}=-K\left(s-s_{0}\right)
$$

Denoting $K, S$, and $S_{0}$ before the decomposition has started by $K^{i}, s^{i}$, $s_{0}^{i}$, one has

$$
\left(c_{2}^{i}-c_{2}^{f}\right) w=-k^{i}\left(s^{i}-s_{0}^{i}\right)
$$

Multiplying eq. $C-11$ by $(1-n)$ and subtracting it from the preceding equation gives entry $1 c$ of table $\mathrm{C}-4$.

The reltion between $d n / d t$ and $P_{2}$ is given in entry $1 d$ of table $c-4$. $-Q\left(T_{R}\right)$ is the enthalpy of decomposition referred to some arbitrary reference temperature $T_{R}, \quad C_{2}^{p}$ is the heat capacity of all the products of the reaction whether they remain in the sample container or not. When the sample container is closed, $c_{2}^{p}=c_{2}^{f}, c_{2}^{i}$ and $c_{2}^{f}$ are constant volume heat capacities, and $-Q\left(T_{R}\right)$ is the energy of decomposition referred to $\mathrm{T}_{\mathrm{R}}$. If one integrates $-\mathrm{P}_{2}+\mathrm{C}_{2} \mathrm{DT}_{2}$ from a time $\mathrm{t}_{i}$ before the decomposition starts to a time $t_{f}$ after the decomposition is over, one obtains for the 


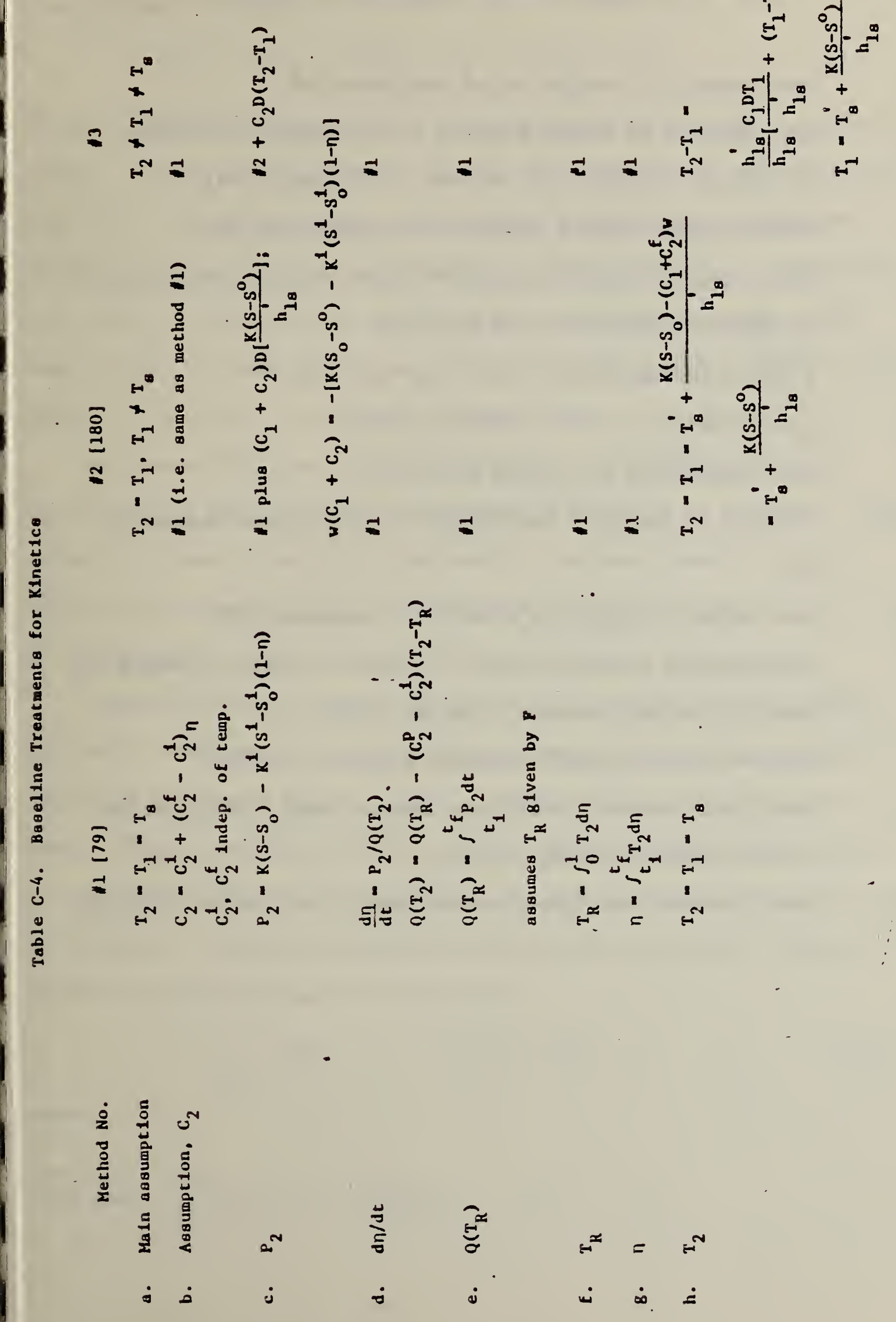


Table C-4a Definitions and Comments, Table C-4

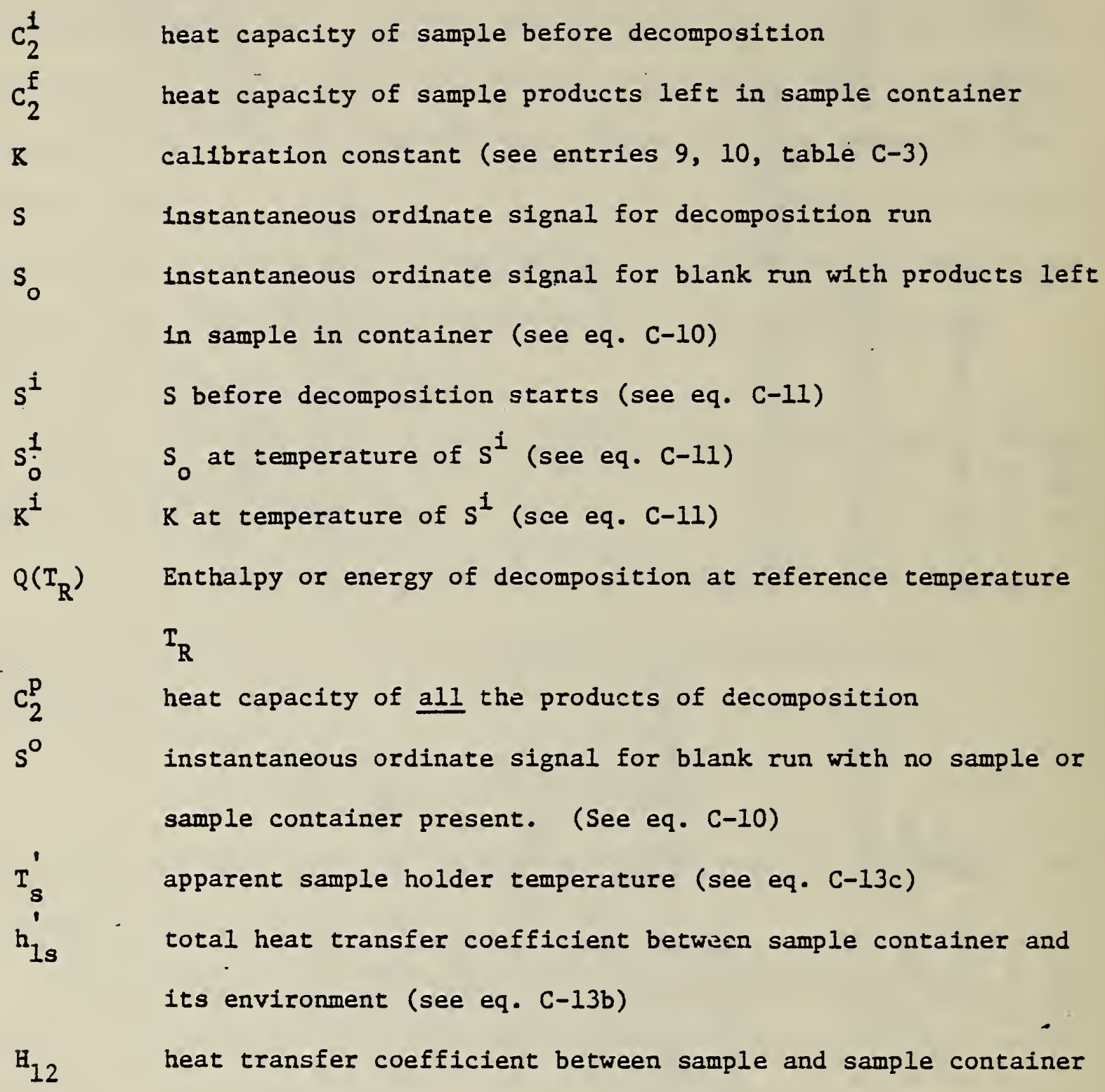


total change in energy of the sample, $\Delta E_{2}$, as expected ${ }^{+}$

$$
\Delta E_{2}=-Q\left(T_{R}\right)+C_{2}^{P}\left(T_{w}^{f}-T_{R}\right)+C_{2}^{i}\left(T_{R}-T_{2}^{i}\right) \quad C-12
$$

$T_{2}^{f}$ and $T_{2}^{i}$ are the temperatures of the sample at times $t_{f}$ and $t_{i}$, respectively. If the sample container is open and products are lost quasistatically, $C_{2}^{f}<c_{2}^{p} ; C_{2}^{p}, C_{2}^{f}$, and $C_{i}$ are constant pressure heat capacities; and $-Q\left(T_{R}\right)$ is the enthalpy of decomposition referred to $T_{R}$. Integrating $-\mathrm{P}_{2}+\mathrm{C}_{2} \mathrm{DT}_{2}$ as before gives the enthalpy of decomposition, $\Delta \mathrm{H}_{2}$. The equation has the same form as eq. $C-12$ for $\Delta E_{2}$ plus $-\left(C_{2}^{p}-C_{2}^{f}\right)\left(T_{2}^{f}-T_{R}\right)$ added on the right to account for product loss.

As Speros [183] has shown, the integral of $P_{2}$ from $t_{i}$ to $t_{f}$ is equal to $Q\left(T_{R}\right)$ entry $1 e$, if $T_{R}$ is defined by entry if of table $C-4$. The value $\eta$ is given by entry $1 \mathrm{f}$. Ordinarily, the heat capacity term is small in comparison to $Q\left(T_{R}\right)$ so that one is required to solve $1 \mathrm{c}$, $1 \mathrm{~d}$, and If iteratively.

General practice aims (or should aim) at insuring that the sample and sample container are in much better thermal contact than the sample container and sample holder. Thus, a somewhat better approximation to entry $1 \mathrm{la}$ is to assume that $T_{1}=T_{2}$, as do Heuvel and Lind [184]. The corresponding entries for this method, $\$ 2$, are listed in entries $2 \mathrm{a}$ to $2 \mathrm{~h}$ of table $\mathrm{C}-4$. To derive entry $2 \mathrm{c}$ one must derive entry $2 \mathrm{~h}$. Adding entries 1 and 2 of table $C-3$ one obtains

$$
\sum_{1,2} C_{i} D T_{i}-P_{2}=h_{I s}^{\prime}\left(T_{s}^{\prime}-T_{1}\right) \quad C-13 a
$$

where

†ne uses: $\int_{T_{2}^{2}}^{T_{2}^{f}} \eta d T_{2}=T_{2}^{f}-\int_{0}^{1} T_{2} d \eta$. 


$$
\begin{gathered}
\hat{h}_{1 s}^{\prime}=h_{1 s}+h_{1 b}+h_{13} \\
T_{s}^{\prime}=T_{s}+h_{1 b}\left(T_{b}-T_{s}\right)+h_{13}\left(T_{3}-T_{s}\right)
\end{gathered}
$$

$\mathrm{T}_{\mathbf{S}}^{\prime}$ is the apparent temperature of the environment of the sample container. Eliminating $\Sigma_{i} C_{1} D T_{i}+P_{2}$ from eq. C-13a and using entry 9 of table $C-3$ gives the first line of entry $2 \mathrm{~h}$ of table $\mathrm{C}-4$. To obtain the second line of entry $2 \mathrm{~h}$, suppose one carries out a new blank run in which neither sample and sample container are present. Replacing $C_{1}, C_{2}, P_{2}$ of entry 6 , table $\mathrm{C}-3$, with zero and assuming $\mathrm{DT}_{S}=\mathrm{DT}_{\mathrm{I}}=w$, etc., as in deriving eq. C-10, one obtains

$$
\left[C_{s}-C_{r}-f_{2}^{\prime}\left(C_{3}+C_{4}\right)\right] w=H^{\prime} f_{d} S^{0}+\Delta h\left(T_{b}-T_{r}\right) \quad C-14
$$

where

$$
\begin{aligned}
& f_{2}^{\prime}=f_{2}+2 h_{13} / h_{r 3} \\
& \mathrm{~B}^{\prime}=\mathrm{B}-\mathrm{h}_{1 \mathrm{~b}}-2 \mathrm{~h}_{13}-\mathrm{h}_{\mathrm{rl}}
\end{aligned}
$$

Subtracting eq. C.-14 from eq. C-10 and assuming $f_{2}^{\prime}-f_{2}$ and $H^{\prime}-H$ are small yields

$$
\left(C_{1}+C_{2}^{f}\right) w=-K\left(s_{0}-s^{o}\right)
$$

Substituting eq. C-15 into the first line of entry $2 \mathrm{~h}$ yields the second line of $2 \mathrm{~h}$. Differentiating the second line of entry $2 \mathrm{~h}$, assuming $D T_{s}^{\prime}=$ $w$, and substituting inco entry 9 of table $C-3$ gives entry $2 c$ of table $C-4$.

.It is useful to note that a third blank run in which no sample is present and for which the instantaneous ordinate signal is $\mathrm{S}_{\mathrm{e}}$ provides a check on the assumption that $C_{2}^{f}$ is independent of temperature if the sample container is closed. One can show

$$
-C_{2}^{f}=-K\left(S_{o}-S_{e}\right)
$$


Since no corresponding measurement of $\mathrm{c}_{2}^{1}$ is possible, the assumption that both $C_{2}^{i}$ and $C_{2}^{f}$ are constant is still necessary in table $C-4$.

The second method requires that one not only determine $K$ over the temperature range of interest but also determine $h_{1 s}^{\prime}$ for the sample container. Usually, $h_{1 s}^{\prime}$ is sufficiently large and the experiment is run so that the correction to $\mathrm{T}_{\mathrm{s}}$ is small. In this case, $\mathrm{h}_{1 \mathrm{~s}}^{\prime}$, for disposable containers of nearly uniform type, can be determined from the melting point-enthalpy of fusion calibration runs of metals used to determine $T_{S}$ and to calibrate $\mathrm{K}$ as a function of $\mathrm{T}_{\mathrm{S}}$. The method is summarized later. To obtain some feeling from the nature of the errors in method 2 , it is useful to tabulate the measurement equations when the temperature of the sample materials, container, and holder are each assumed to be uniform but unequal. These are given in table $\mathrm{C}-4$ under method 3 . The addition to entry $3 \mathrm{~b}$ is $\mathrm{C}_{2} \mathrm{D}\left(\mathrm{T}_{2}-\mathrm{T}_{1}\right)$ where $\mathrm{T}_{2}-\mathrm{T}_{1}$ is given in entry $3 \mathrm{~h}$. It is evident that for a given $\left(\mathrm{C}_{1} \mathrm{DT}_{1} / \mathrm{h}_{1 \mathrm{~s}}^{\prime}+\mathrm{T}_{1}-\mathrm{T}_{\mathrm{s}}\right)$ that $\mathrm{T}_{2}-\mathrm{T}_{1}$ is made smaller as $\mathrm{h}_{1 s^{\prime} / \mathrm{h}_{12}}$ decreases. Another way of examining entry $3 \mathrm{~h}$ is to note that $\mathrm{T}_{2}-\mathrm{T}_{1}$ is given by

$$
T_{2}-T_{1}=\frac{P_{2}-C_{2} D T_{2}}{h_{12}}=\frac{V\left(Q d \eta / d t-C_{2} d T_{2} / d t\right)}{A \alpha_{0}}
$$

$Q$ and $c_{2}$ are the heat of reaction and heat capacity, respectively, per. unit volume of sample. $V$ is the volume of sample and $A$ is the area of contact of sample and sample container having a heat transfer coefficient per unit area of $a_{0}$. Assuming $Q$ and $c_{2}$ are constant and that the sample is a thin layer of thickness $2 \mathrm{r}$ and area $A, \mathrm{~T}_{2}-\mathrm{T}_{1}$ can be minimized by reducing $2 r / \alpha_{0}$. This is done in the proposed ASTM E-27, test [153] by sandwiching a thin layer of sample between the container bottom and lid 
so $\mathrm{V} / \mathrm{A \alpha} \alpha_{0}$ equals $\mathrm{r} / \alpha_{0}$. Since $\mathrm{T}_{1}-\mathrm{T}_{\mathrm{s}}$ is given by

$$
\mathrm{T}_{1}-\mathrm{T}_{\mathrm{s}}=\frac{\mathrm{P}_{2}}{\mathrm{~h}_{1 \mathrm{~s}}^{\prime}}-\frac{\mathrm{C}_{1} \mathrm{DT} \mathrm{T}_{1}+\mathrm{C}_{2} \mathrm{DT} \mathrm{T}_{2}}{\mathrm{~h}_{1 \mathrm{~s}}^{\prime}}
$$

reducing $h_{1 s}^{\prime}$ increases $T_{1}-T_{s}$ which reduces temperature gradients in the sample as discussed in Appendices B.6., B.10., and C.1. The main problem in adopting the procedure of reducing $h_{1 s}^{\prime}$ is that the inaccuracy with which $h_{1 s}^{\prime}$ is known must correspondingly decrease. Preferably a procedure must be devised to determine $\hat{h}_{1 s}^{\prime}$ of each sample container with the sample in place prior to the decomposition run.

Method 3 of table C-4 clearly cannot be used to evaluate $\mathrm{T}_{2}$ unless $\mathrm{h}_{12}$ is known during the decomposition. There is no method for determining $\mathrm{h}_{12}$ from $\mathrm{s}$ under these conditions.

\section{C.3.3. Measurement Equations for DSC}

The instrument used in our experimental work is a commercial unit made by Perkin-Elmer Corporation, Model DSC-2. It consists of two separate calorimeters $^{+}$which are mounted on supporting tubes in separate cylindrical cavities in an aluminum block, E of figure 4 (reproduced here for convenience). The instrument differs from the Boersema type (heated block) of twin scanning calorimeter of figure 3 in that the block is thermostatted at or near room temperature while the calorimeters are operated at the temperature or in the temperature range of interest. In other words, if the temperature of the instrument is set at $500 \mathrm{~K}$, the temperature difference between either calorimeter and its immediate environment is of the order of $500-300=200 \mathrm{~K}$. This temperature difference

\footnotetext{
The sample calorimeter is sample, sample container, and sample holder.
} 
produces larger temperature gradients in both calorimeters than would be present if the block temperature was nominally equal to that of the calorimeters. However, these temperature gradients can automatically be taken Into account to a good first approximation by calibrating the energy and temperature scales of the instrument using the same thermal geometry inside the calorimeter (i.e. same sample containers, same holder lids, etc) for both the calibration and "unknown" experiments" . This first design feature permits the calorimeters to be cooled, other things being equal, more rapidly than in the Boersema design (i.e. the latter requires cooling of the block also.)

The second difference in the design of this instrument from the typical commercial unit based on the Boersema design is that the difference between the sample holder temperature, $\mathrm{T}_{S}$, and reference holder temperature, $T_{I}$, which are sensed by the platinum resistors $S_{T h}$ and $R_{T h}$, respectively, is not the ordinate output signal, $\mathrm{S}$, of the calorimeter. Instead, electrical power is supplied to the sample and reference calorimeter heaters, $S_{h}$ and $R_{h}$, respectively, to keep $T_{S}$ and $T_{I}$ nominally equal. $S$ is proportional to the difference between the power supplied to the calorimeters to maintain $T_{S}$ and $T_{r}$ nominally equal. An additional increment of electrical power is supplied to both calorimeters to keep $T_{s}$ and $T_{r}$; nominally equal to the program temperature, $T_{p}$, of the instrument.

To accomplish this type of operation when an exothermic reaction takes place in the sample calorimeter requires some way of withdrawing heat rapidly from this calorimeter. The large temperature difference takes care of this ${ }^{+}$.

\footnotetext{
${ }_{\text {This }}$ is an intuitive observation based on the study cited in Appendix C. 4. + Rapid cooling is achieved in some heated block (Boersema type) calorimeters by using a Peletier cooler.
} 
The particular features of the design of the instrument that are relevant to how an experiment is carried out are as follows. Referring to figure 4 , each calorimeter holder consists of a cup, $S_{c}$ or $R_{c}$, with a platinum lid, $S_{e}$ or $R_{1}$, that can be removed to insert samples, 2 and 4, enclosed by their containers, 1 and 3, into the sample and reference holders. The heater and temperature sensor of each holder are sandwiched between metal plates that form the bottom of the cup of each holder. Accnrding to the patent description [174], these plates, the wall of the cup, and the tube supporting the calorimeter are made of $\mathrm{Pt}-20 \%$ Ir which are electron-beam welded into a single unit. The heater and sensor consist of identical resistors made by winding (nonbifilarly) $0.005 \mathrm{~cm}$ diameter platinum wire on a thin rectangular chip of alumina. The wire is insulated from the adjacent metal plates by thin alumina cloth. Thermal contact of the resistor wires with the cup occurs through the cloth. Possible air gaps between the cloth and wire or cloth and plates, which would reduce thermal contact of the wires with the cup, is avoided by welding the plates in position after the sensor and heater have been compressed between the plates. thus, the construction reduces the temperature gradients in the cup and insures that the temperature of the sensor and heater are. near that of the cup. The use of platinum, platinum iridium alloy, and alumina produce an oxidation resistant, physically stable (i.e. nearly uniform coefficient of expansion) calorimeter.

In the usual experiment, the sample container is in good thermal contact with the sample, and, also, it is in much better thermal contact with the calorimeter cup than the calorimeter lid. Thus, to a first approximation, sample, sample container, calorimeter cup, calorimeter heater, and calorimeter temperature sensor all are near the same temperature. 


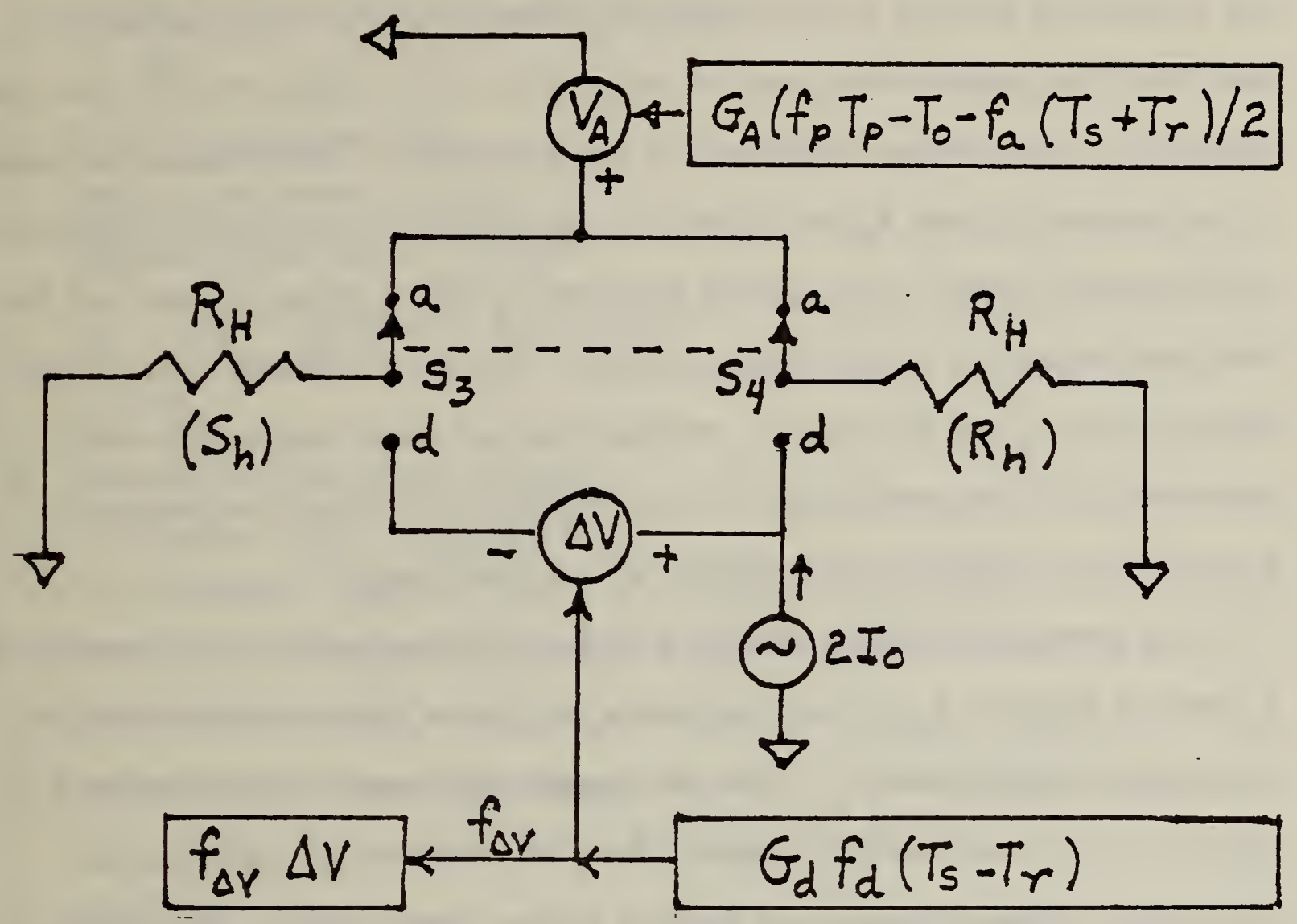

Figure C-3. Simplified Sketch of DSC-2 Output Circuitry

a, heaters connected to average amplifier circuit; d, heaters connected to difference amplifier circuit; $f_{a}$, proportionality factor of average temperature detection circuit; $f_{p}$, adjustable parameter of program temperature source; fd, proportionality factor of difference temperature detection circuit; $f_{\Delta V}$, proportionality factor of energy (ordinate) output circuit; $G_{A}$, gain of average amplifier circuit; $G_{d}$, gain of difference amplifier circuit, $2 I_{0}$, magnitude of constant current source; $R_{y}$, heater resistance; $\left(R_{h}\right)$, reference holder heater; $S_{3}$ gauged heater switches; $\left(S_{h}\right)$ sample holder heater; $T$, program temperäture; $T$, adjustable parameter of program temperature source; $I_{s}$, sample holder temperature; $T$, reference holder temperature; $\bar{\Delta} \mathrm{V}$, disference cirucit voltage source ${ }^{\Gamma} \mathrm{V}_{A}$, average circuit voltage source. 
An understanding of how the compensation circuitry works can be developed by first considering the operation of the output power circuitry and neglecting details of the input or temperature detection circuitry. Combining the appropriate parts of refs. $[154,175,178]$, one can view the operation of the output circuitry as the continuous, simultaneous switching of the heaters $S_{h}$ and $R_{h}$ of figure $C-3$ alternately to the power output of an averaging circuit (e.g. ganged switches. $S_{3}$ and $S_{4}$ up as shown) and to the power output of a difference circuit. $2 I_{0}$ is a constant D.C. current source while $V_{A}$ and $\Delta V$ are D.C. voltage sources whose magnitudes are represented by the expressions in the appropriate "input" rectangles. $\mathrm{f}_{\Delta \mathrm{V}} \Delta \mathrm{V}$ is the ordinate output signal of the instrument.

The averaging circuit detects a signal proportional to the average of $T_{s}$ and $T_{r}, f_{a}\left(T_{S}+T_{r}\right) / 2$, and subtracts it from a signal proportional to the program temperature $T_{p}$. The net signal is Iinearly amplified by a factor $G_{A}$ and supplied as voltage across both heaters to minimize the difference between program and average holder temperatures. The corresponding time average power, $\mathrm{P}_{A}$, suppplied to each holder by the average circuit, assuming $\mathrm{V}_{\mathrm{A}}$ is an ideal source and the resistance of each heater. equals $R_{H}$, is given by entry 1 of table $C-5$. The parameters $f_{p}$ and $T_{0}$ of the input signal are adjusted in calibrating the temperature scale while the parameter $f_{a}$ is, in effect, controlled to be independent of temperature. The difference circit detects a signal proportional to $T_{S}{ }^{-T}$ that is linearly amplified by a factor $G_{d}$ and appears as a voltage in series with the holder heaters to keep the difference in holder temperatures to a minimum. 
Table C-5. Equations or S1mplified DSC Measurement Analysis

1. Average Amplifter Power

$P_{A}=\frac{1 V_{A}^{2}}{2 R_{B}} ; V_{A}=G_{A}\left[f_{P} T_{P}-T_{0}-E_{a}\left(\frac{T_{S}+I_{I}}{2}\right)\right]$

2. Difference Amplffier Power

Sample holder power: $P_{B}-P_{D} / 2$

Reference holder power: $P_{B}+P_{D} / 2$

$P_{B}=\frac{1}{2}\left[I_{0}^{2}+\frac{\Delta V^{2}}{4 R h}\right] R_{H} ; \Delta V=G_{d} E_{d}\left(T_{S}-T_{I}\right)$

$P_{D}=\Delta V I_{0}$

3. Energy balance for sample contafner
a. sample: $C_{2} D T_{2}=h_{21}\left(T_{1}-T_{2}\right)+P_{2}$
b. container: $C_{1} D T_{1}=h_{12}\left(T_{2}-T_{1}\right)+h_{1 s}\left(T_{s}-T_{1}\right)+h_{1 l}\left(T_{2}-T_{1}\right)$
c. bolder 11d: $C_{2} D T_{2}=h_{2 s}\left(T_{s}-T_{l}\right)+h_{21}\left(T_{1}-T_{2}\right)+\dot{Q}_{l}$
d. holder cup: $C_{s} D T_{s}=h_{s l}\left(T_{\ell}-T_{s}\right)+h_{s l}\left(T_{s}-T_{1}\right)+\dot{Q}_{s}+P_{A}+P_{B}-\frac{1}{2} P_{D}$

4. Energy balance: sample calortmerer

$$
\Sigma_{1} \quad C_{1} D T_{1}-P_{2}=\ddot{Q}_{3}+P_{A}+P_{B}-\frac{1}{2} P_{D} ; \ddot{Q}_{3}=\dot{Q}_{S}+\dot{Q}_{2}
$$

$1=1,2,3,2$

5. Energy balance: reference calortmeter

$$
\Sigma_{1} \quad C_{1} D T_{1}=\ddot{Q}_{I}+P_{A}+P_{B}+\frac{1}{2} P_{D} ; \ddot{Q}_{I}=\dot{Q}_{I}+\dot{Q}_{D}
$$

$1 \cdot 3,4,5, m$

6. Energy measurement equation

$$
\Sigma_{1} \quad C_{1} D T_{1}-\Sigma_{1} \quad C_{1} D r_{1}-P_{2}=\ddot{Q}_{s}-\ddot{Q}_{E}-R S ; S=\Delta V \varepsilon_{\Delta V} ; R=\frac{I_{0}}{E_{\Delta V}}
$$

$1=1,2, s, 2$

$$
3,4,5, m
$$

7. Energy equation for blank run with decomposition products in sample container

$$
\left[\Sigma_{1} c_{1}+c_{2}^{f}-\Sigma_{1} c_{1}\right]_{w}=-k S_{0}+\ddot{Q}_{s}-\ddot{Q}_{T}
$$

$$
1=3,2,1 \quad r, m, 3,4
$$

8. Energy measurement equation for decomposition run minus blank with products

$$
\begin{aligned}
& \left.\Sigma_{1} C_{1} D T_{1}-\left(C_{1}+C_{2}^{f}\right) w-P_{2}=-K\left(S-S_{0}\right) ; D T_{1} \text { (decomp. }\right)=D T_{1} \text { (blank) }=w ; \\
& 1,2 \text { 1 } 1=8, I, 3,4, l, m \\
& \ddot{Q}_{s}-\ddot{Q}_{5} \text { same in decomp. and blank }
\end{aligned}
$$


The corresponding time average power, $\left(P_{B} \pm P_{D}\right) / 2$, supplied to each holder by the difference circuit is given in entry 2 of table C-5. It is assumed both $\Delta V$ and $2 I_{0}$ are ideal sources and again that the resistances of the heaters are equal to $R_{H}$. The parameter $f_{d}$ of the input signal is temperature dependent.

The basic measurement equations are obtained as in the QDTA instrument analysis. The energy balance equations for the sample, sample container, sample holder lid, and sample holder cup are given in entries $3 a, 3 b, 3 c$, and $3 \mathrm{~d}$, respectively. $C_{\ell}$ and $T_{\ell}$ are the heat capacity and temperature respectively, of the sample holder 1 id. $h_{1 \ell}$ and $h_{\ell s}$ are the heat transfer coefficients between the 1id and sample holder and the lid and the sample holder cup, respectively. $-\dot{Q}_{\ell}$ is the rate of heat loss to the block from the lid. The subscript $s$ is used to denote the sample holder cup; $-\dot{Q}_{s}$ is the rate of heat loss to the block from the cup. Definitions of the other heat capacities and heat transfer coefficients are the same as in table C-3 for the QDTA instrument.

The total energy balance for the entire sample holder plus contents is given in entry 4. The corresponding energy balance for the reference holder is given in entry 5 . The subscripts $m$ and $r$ refer to the sample holder lid and cup, respectively.

Subtracting entry 5 from entry 4 yields entry 6 . Entry 6 of table C-5 is formally identical with entry 6 of table C-3 for the QDTA instrument. $C_{s} D T_{s}, C_{s} D T_{I}, E_{1}, f_{3}$, and $H f_{d}$ of the QDTA equation are replaced by $C_{s} D T_{s}+$ $C_{\ell} \mathrm{DT}_{\ell}, C_{r} D T_{I}+C_{m} D T_{m}, 1,1$, and $k$, respectively. In the expression for the calibration constant, $K$, of the DSC, $f_{\Delta V}$ represents the gain of the electrical circuit. Since both $I_{0}$ and $f_{\Delta V}$ are constant, $K$, in this 
instrument is independent of the program temperature $T_{p}$. Subtracting the energy equation for a blank run with sample products in the sample container, entry 7, from entry 6 yields entry 8 which formally corresponds to entry 9 in table $\mathrm{C}-3$.

The assumption that $\mathrm{DT}_{\ell}$ and $\ddot{Q}_{S}-\ddot{Q}_{I}$ are the same for the decomposition and blank runs in entry 9 are interrelated since $\dot{Q}_{s}^{\prime}=\dot{Q}_{s}+\dot{Q}_{\ell}$ and $\dot{Q}_{2}$ depends upon $\mathrm{T}_{\ell}$, the temperature of the sample holder lid. Qualitatively, the assumption that $\mathrm{DT}_{\ell}$ and $\dot{Q}_{\ell}$ are the same in both runs requires that the rate of heat transfer between, the holder lid and sample container be much less than the total rate of heat transfer between the holder Iid and the holder cup and the holder lid and the block.

The equations in table C-4 apply equally well to the DSC instrument. The only essential differences are that now ' ${ }_{s}$ ' $h^{\prime}{ }_{1 s}$, and the energy equation for $S^{\circ}$ (see columns 2 and 3 ) are:

$$
\begin{aligned}
& h_{1 s}^{\prime}=h_{1 s}+h_{1 l} \\
& \text { C-16 } \\
& T_{s}^{\prime}=T_{s}+\frac{h_{1 \ell}}{h_{1 s}^{\prime}}\left(T_{\ell}-T_{s}\right) \\
& {\left[\Sigma_{i} C_{i}-\Sigma_{i} C_{i}\right] w=-\mathrm{K}\left(S-S^{0}\right)+\ddot{Q}_{S}-\ddot{Q}_{I}} \\
& \text { C-16c } \\
& \mathrm{s}, \mathrm{l} \quad \mathrm{r}, \mathrm{m}, 3,4
\end{aligned}
$$

As for the QDTA instrument, eq. 6 of table $C-5$ and the equations of table C-4 apply only after starting transients have decayed out.

\section{C.3.4. Transfer Functions and Temperature Calibration}

The relation between $S$ (instantaneous signal) and 0 (observed signal) for the DSC instrument was first investigated by Flynn [190] who measured the time response of 0 caused by exposing the empty sample holder cups of an earlier model to infrared heat pulses. It was found that 


$$
S=\left(1+\tau_{d} D\right) 0
$$

$\tau_{d}$ is the longest time constant of the two holder cups and lids, holder heaters and difference power circuit to the extent that the rise time of $P_{2}$ caused by the pulses is small in comparison to $\tau_{d}$. (An assumption of this type or appropriate modeling is always required to relate the known power input to $\mathrm{P}_{2}$ ) Another method is to determine the time behaviour of 0 caused by the very rapid rate of liberation of heat caused by the freezing of a supercooled liquid metal [191, p. 33-39]. The assumption is that $S$ due to freezing has a rise time much less than $\tau_{d}$. The preferred direct method of determining the ordinate transfer function is to determine the time response of 0 due to electrical heating.

For calorimeters having considerably greater stability and precision than our instrument, an expression of the form

$$
S=\left(1+\Sigma_{i} \tau_{i} D^{i}\right) 0 \quad C-17 b
$$

is used. The more detailed experimental procedures and analyses required for eq. C-17b are discussed in references [192-196]. Equation C-17b does not seem to be warranted for the relatively slow changes in 0 encountered in our decompositions run in the scanning mode and the present uncertainties in realizing assumptions of procedures or commercial instruments discussed here. In addition, the methods seem inappropriate because - electrical calibration experiments would be very difficult for the commercial apparati considered here and also modeling would be required since the thermal time constant of the electrical calibration heater system could not be neglected in comparison to $\tau_{d}$. 
The instantaneous abscissa signal of the DSC and QDTA instruments described here are proportional to $\mathrm{T}_{\mathrm{p}}$ and $\mathrm{T}_{\mathrm{r}}$, respectively. For the DSC, following Flynn [189], we assume $\mathrm{T}_{s}^{\prime}$ is related to $\mathrm{T}_{\mathrm{p}}$ in a scanning experiment by

$$
\mathrm{T}_{s}^{\prime}-\mathrm{T}_{\mathrm{p}}=\Delta \mathrm{T}_{\mathrm{STAT}}-\tau_{\mathrm{a}} \mathrm{w}
$$

where $\Delta \mathrm{T}_{\mathrm{STAT}}$ is the static difference between $\mathrm{T}_{\mathrm{S}}^{\prime}-\mathrm{T}_{\mathrm{p}}$ when the calorimeter is operated in the isothermal mode $(w=0) . \tau_{a}$ is the longest time constant of the holder cups and lids, holder heaters, and the averaging power circuit.

$\Delta \mathrm{T}_{\text {STAT }}$ can be determined directly [185] by determining the smallest nominal temperature, $T_{p}\left(t_{1}\right)$, for which a melting or transition temperature standard begins to absorb heat if the temperature is stepped to $T_{p}\left(t_{2}\right)$ where $T_{p}\left(t_{2}\right)-T_{p}\left(t_{1}\right)$ is the resolution of the programer of the instrument $(0.1 \mathrm{~K}$ for the DSC-2).

$$
\Delta T_{\text {STAT }}, \tau_{a}+\tau_{d} \text {, and } h_{1 s}^{\prime} \text { (needed to evaluate entry } 2 h \text { of table } c-4 \text { ) }
$$
can be determined from variation of the apparent melting or transition point, the so-called extrapolated onset temperature, of the material as a function of scanning rate. The extrapolated onset temperature, $T_{\text {on }}(w)$, is constructed from a sketch of the observed output signal, 0 , from an Indium melting point standard in figure $\mathrm{C}-4$. The argument used to interpret the variation of $\mathrm{T}_{\text {on }}(w)$ is as follows. Suppose first that the sample both before and during the transition is in good thermal contact with its container so that $\mathrm{T}_{2}=\mathrm{T}_{1}$. Then entry $2 \mathrm{~h}$ of table $\mathrm{C}-4$ will apply provided we replace $s$ by $s^{i}$ and $c_{2}^{f}$ with $c_{2}^{i}$ :

$$
\mathrm{T}_{2}=\mathrm{T}_{1}=\mathrm{T}_{\mathrm{s}}^{\prime}+\frac{\mathrm{K}}{\mathrm{h}_{1 \mathrm{~s}}^{\prime}}\left[\mathrm{S}-\mathrm{S}^{i}\right]-\tau_{\mathrm{s}}^{i} \mathrm{w}_{\mathrm{s}}^{i}=\frac{\mathrm{C}_{1}+\mathrm{c}_{2}^{i}}{\mathrm{~h}_{1 \mathrm{~s}}}
$$




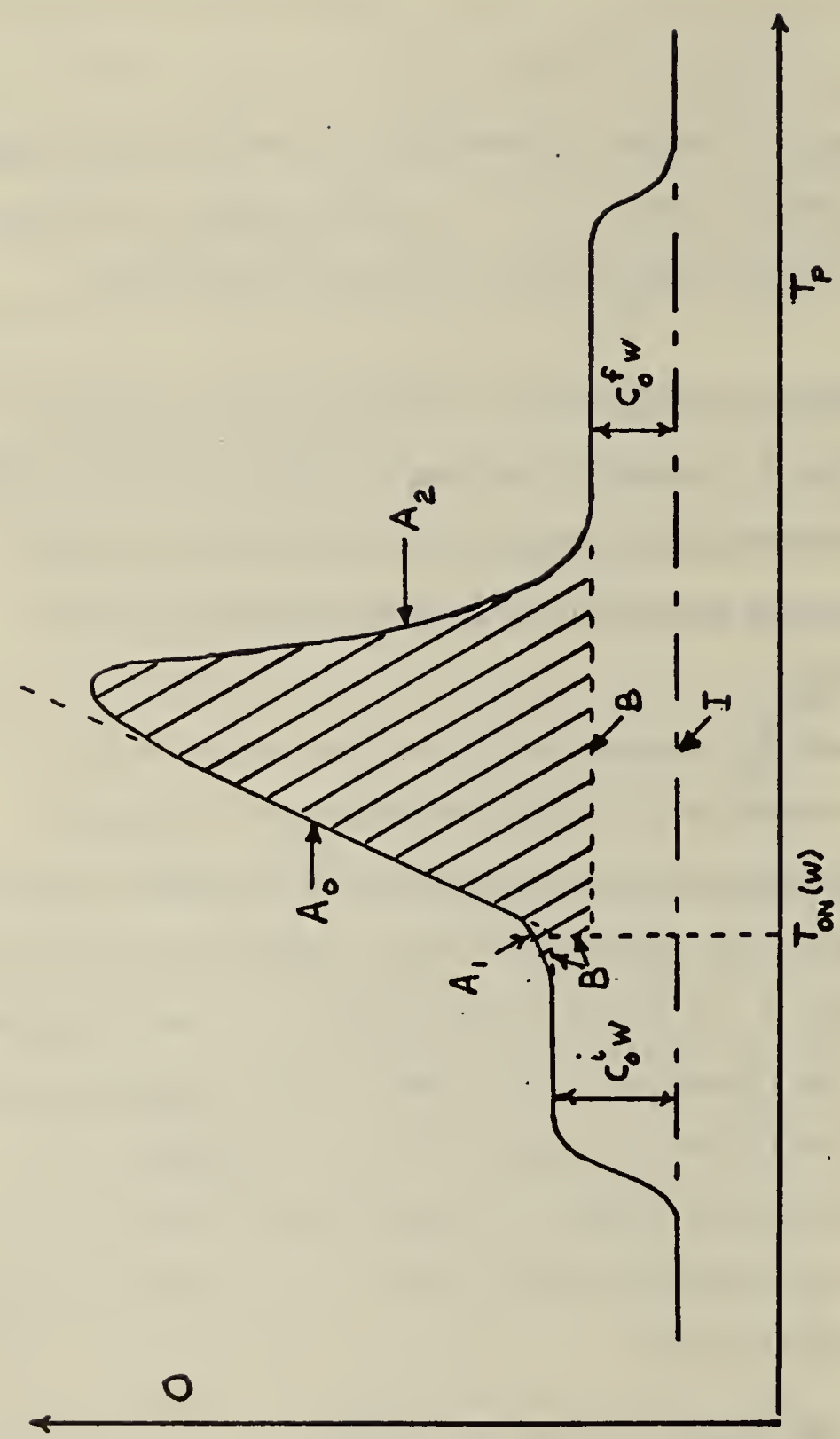

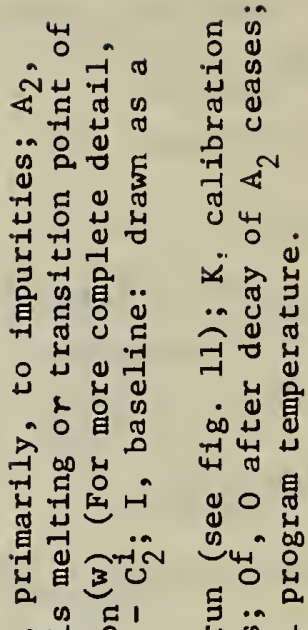
की संफुत मे की ना

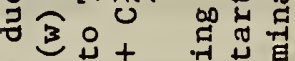

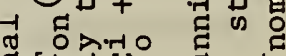

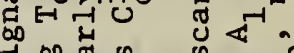

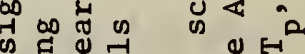

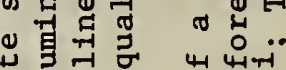

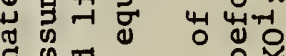
की

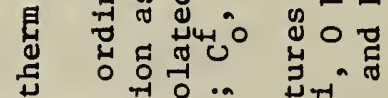

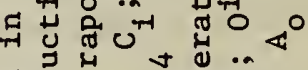

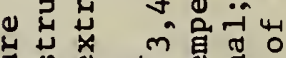
出 ग 0 एँ

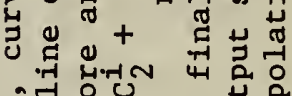
ज氙艺 光

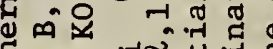

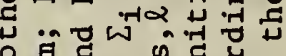
ठृ

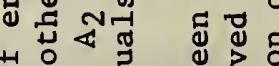

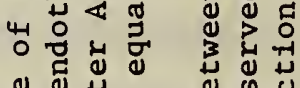
य ग पृ山्यु ( 4

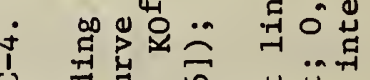

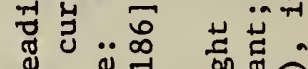

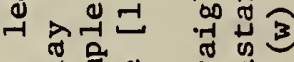

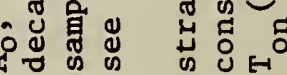


Inserting eqs. $\mathrm{C}-17 \mathrm{a}$ and $\mathrm{C}-18$ into eq. $\mathrm{C}-19$ gives

$$
T_{2}=T_{p}+\Delta T_{S T A T}-\tau_{a} w+\frac{K}{h_{1 s}^{\prime}}\left(1+\tau_{d} D\right)\left(0-0^{i}\right)-\tau_{s}^{i}
$$

Just after melting starts $\mathrm{K}\left(0-0^{i}\right)$ is observed to decrease linearly with time at a rate $\alpha$ so

$$
K\left(0-0^{i}\right)=-\alpha\left[T_{p}-T_{\text {on }}(w)\right] ; T_{p}>T_{\text {on }}(w)
$$

Inserting the last relation with eq. C-20a gives

$$
T_{2}=T_{p}+\Delta T_{S T A T}-w \tau_{a}-\frac{\alpha}{h_{1 s}^{\prime}}\left[T_{p}-T_{\text {on }}(w)\right]-\tau_{d} \frac{\alpha}{h_{1 s}} w-\tau_{s}^{1} w ; T_{p} \geq T_{\text {on }}(w)
$$

Assuming $\mathrm{T}_{2}$ is constant and equal to the transition or fusion temperature, $\mathrm{T}_{\text {tr }}$, for $\mathrm{T}_{\mathrm{p}} \geq \mathrm{T}_{\text {on }}(\mathrm{w})$, one has from the condition that $\mathrm{DT}_{2}=0$ that $\alpha$ equals $h_{1 s}^{\prime}$. Inserting this value for $\alpha$ in eq. $\mathrm{C}-20 \mathrm{~b}$ and rearranging we have the results

$$
\begin{array}{cc}
\alpha=h_{1 s}^{\prime} & c-21 a \\
T_{\text {OD }}(w)=T_{t I}-\Delta T_{\text {STAT }}+\tau_{0} w & c-21 b \\
\tau_{0}=\tau_{a}+\tau_{d}+\tau_{s}^{\perp} & c-21 c
\end{array}
$$

Thus, $h_{1 s}^{\prime} w$ is the slope of the leading edge of $0-0^{i}$ divided by $k, \Delta T_{S T A T}$ equals $T_{t r}-T_{\text {on }}(0)$, and the slope of a plot of $T_{\text {on }}(w)$ versus $w$ should be constant and equal to $\tau_{a}+\tau_{d}+\tau_{s}$. These results were first cited by Flynn [189]. Experimentally, it is found that for very pure metal meltIng point standards (e.g. see $[187,188,189])$, the slope of $T_{\text {on }}(w)$ does vary linearly with $\mathrm{w}$ and the value of $\Delta \mathrm{T}_{\text {STAT }}$ seems to agree within a few tenths of a Kelvin with the direct method cited above. 
The value of $\tau_{s}^{i}$ can be eliminated by determining $\tau_{0}$ for a cooling run (see [189]) or by determining the decay constant, $\tau_{s}^{f}$, of the observed decay curve of the endotherm. Since $\tau_{s}^{f}$ for $T_{1}=T_{2}$ is

$$
\tau_{s}^{f}=\frac{c_{1}+c_{2}^{f}}{h_{1 s}}
$$

$\tau_{s}^{f}$ equals $\tau_{s}^{i}$ if $c_{2}^{f} \ll C_{1}$, the usual situation in practice.

Another method for determining $\tau_{a}$ is given in the next section.

To evaluate the temperature in a decomposition run one uses eq. $20 \mathrm{a}$ with $\tau_{s}^{i}$ replaced by $\tau_{s}^{f}$ but now $C_{2}^{f}$ is the heat capacity of the decomposition products and $0^{1}$ replaced by $0_{0}$

$$
\begin{gathered}
T_{2}=T_{1}=T_{p}+\Delta T_{\text {STAT }}-\left(\tau_{a}+\tau_{s}^{f}\right) w+V \\
V=\frac{K}{h_{1 s}^{\prime}}\left(1+\tau_{d} D\right)\left(0-0_{0}\right)
\end{gathered}
$$

$\nabla$ is called the correction for self heating. In our decomposition runs, $\mathrm{T}_{2}$ is calculated for the value of $\mathrm{T}_{\mathrm{p}}$ when $0-\mathrm{O}_{0}$ is a maximum or when $\mathrm{D}(\mathrm{O}-$ $\left.0_{0}\right)=0$. Inserting this condition into eq. C-22b yields eq. 16 of the text since in our decomposition runs $c_{2}^{f} \ll c_{1}$.

It is pertinent to note that if sample containers of the same type and weight are used in both the decomposition runs and the dynamic temperature calibration procedure discussed above (so that $C_{1}$ and $h_{1}^{\prime}$ are the same in both types of run), some economy in determining the corrections on the right side of eq. $c-22 a$ can be achieved. If $c_{2}^{i}, c_{2}^{f}$ $\ll \mathrm{C}_{1}$ in the temperature runs, $\Delta \mathrm{T}_{\mathrm{STAT}}$ from eq. $\mathrm{C}-21 \mathrm{~b}$ can be inserted in eq. C-22a to obtain

$$
T_{2}=T_{1}=T_{p}+\left[T_{t r}-T_{o n}(w)\right]+\tau_{d} w+V
$$


Thus, if $\tau_{d} w$ is small in comparison to the other corrections, $T_{2}-T_{p}-V$ is the value of $T_{t r}-T_{\text {on }}(w)$ at $T_{p}$. This value is taken from a plot of $\mathrm{I}_{\mathrm{tr}} \mathrm{T}_{\text {on }}(\mathrm{w})$ versus the nominal temperature constructed from the temperature calibration runs for the $w$ corresponding to that of the decomposition run. This procedure is essentially that of ASTM method E 474 which is a part of the proposed ASTM Kinetic Method.

\section{C.3.5. Determination of $\mathrm{K}$}

Calibration of both instruments is essentially the same. The details will be different since $\mathrm{K}$ for the $\mathrm{DSC}-2$ does not vary more than a percent or so with temperature while it does for the QDTA instrument.

As discussed by Flynn [185], the value of $\mathrm{K}$ can be determined independent of time constant considerations by determining the time integral of 0 from one constant nominal temperature, $T_{p}^{i}$, to another temperature $T_{p}^{f}$ of the sample holder for a material whose enthalpy increment, $\mathrm{H}_{2}\left(\mathrm{~T}_{2}^{\frac{f}{2}}\right)$ $\mathrm{H}_{2}\left(\mathrm{~T}_{2}^{i}\right)$ is known. If one subtracts the corresponding time integral of a blank signal, $o_{e}$, when only the container is in the sample holder, one obtains from entry 6 of table $C-5$ and eq. C-17a

$$
\begin{gathered}
\int_{i}^{t}\left[C_{2} D T_{2}-P_{2}\right] d t=H_{2}\left(T_{2}^{f}\right)-H_{2}\left(T_{2}^{i}\right)=-K \int_{t_{i}}^{t}\left(0-\theta_{e}\right) d t \\
T_{2}^{k}=T_{p}^{k}+\Delta T_{S T A T}^{k}, k=i, f
\end{gathered}
$$

It is assumed that the baseline has been adjusted so $0^{f}-0_{e}^{f}-\left(0^{i}+0_{e}^{i}\right)$ is zero. Also, $T_{p}$ should be varied between $T_{p}^{i}$ and $T_{p}^{f}$ in the same way for both runs so that $\ddot{Q}_{S}^{\prime}-\ddot{Q}_{r}^{\prime}$ for blank and sample runs cancel ${ }^{+}$. Operation of

It is also assumed that the integral of the starting and stopping transients not shown in entry 6 of table $\mathrm{C}-5$ cancel between measurement and blank runs. 
the instrument in this way is called the step jump procedure. Both heat capacity standards (e.g. sapphire) or materials whose heat of fusion or transition, $\Delta \mathrm{H}_{\mathrm{tr}}$, may be used to determine $\mathrm{K}$. For a heat capacity calibration

$$
\Delta \mathrm{H}_{2}=\mathrm{H}_{2}\left(\mathrm{~T}_{\mathrm{p}}^{\mathrm{f}}\right)-\mathrm{H}_{2}\left(\mathrm{~T}_{\mathrm{p}}^{i}\right)+\Delta
$$

while for a fusion or transition run

$$
\Delta H_{2}=\Delta H_{t r}+C_{2}^{i}\left(T_{t r}-T_{p}^{i}\right)+C_{2}^{f}\left(T_{p}^{f}-T_{t r}\right)+\Delta
$$

where

$$
\Delta=C_{2}^{f} \Delta T_{S T A T}^{f}-C_{2}^{i} \Delta T_{S T A T}^{i}
$$

$\Delta \mathrm{T}_{\text {STAT }}$ need not be known when $\Delta$ is small in comparison to the nominal enthalpy increment. Neither $\Delta \mathrm{T}_{\mathrm{STAT}}$ or $\mathrm{C}_{2}$ need be known for fusion or transition measurements if $\mathrm{C}_{2}^{k}\left(\mathrm{~T}_{\mathrm{p}}^{\mathrm{f}}-\mathrm{T}_{\mathrm{p}}^{i}\right), \mathrm{k}=\mathrm{i}, \mathrm{f}$, is small in comparison to $\Delta \mathrm{H}_{\mathrm{tr}}$.

$\mathrm{K}$ can also be determined independent of time constant considerations and the temperature scale calibration by using fusion or transition standards and running the container with sample and empty container in the scanning mode through the transition. As outlined by Flynn [185] and Gutmann and Flynn [186], the times $t_{i}$ and $t_{f}$ of eq. C-23a refer, respectively, to times before and after the transition in which the material has reached a quasi-steady state. The added assumption is that the temperature corrections due to running in the scanning mode of the sample container and the sample in both runs at times $t_{i}$ and $t_{f}$ (and only these times) contribute negligible error in evaluating eq. C-23b. This occurs if $C_{1}$ is arranged to be much greater than $C_{2}^{i}$ or $C_{2}^{f}$, or if $C_{2}^{i}$ nearly equals $C_{2}^{f}$. The alternative procedure is to evaluate the cross hatched area of figure C-4. As Gutmann and Flynn [186] have shown, this area equals, $\Delta \mathrm{H}_{t r}$ If the onset of the transition occurs at $\mathrm{T}_{\text {on }}(w)^{+}$.

\footnotetext{
This can be derived using eqs. C-19, C-17a.
} 
An alternative method for determining. $\tau_{\text {a }}$ that does not depend on the dynamics of a transition or fusion has been developed by Richardson et al [187, 188]. They determined the temperature lag of different weights of sapphire (heat capacity standard) from the area under the total area of a $0-O_{e}$ versus $T_{p}$ curve and the area under the stopping transient when heat capacities are measured by the step jump procedure outlined previously. No container is used, material is in the form of rods, and it is arranged that $h^{\prime}{ }_{l s}$ is as large and constant as possible for each sample weight. $\tau_{\mathrm{a}} \mathrm{w}$ is obtained by extrapolating the temperature lag of the various sample weights at the same $w$ to zero sample weight. This method gives results that are consistent with the dynamic method cited in section C. 3.4 . 


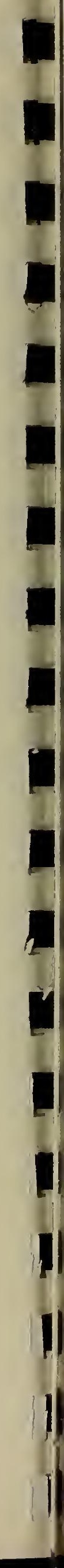




\section{C.4. Instrument Calibration \\ C.4.1. Main Experimental Results}

The temperature scale of the DSC-2 instrument was calibrated with solid holder covers in place using disposable unsealed crimped containers (Perkin-Elmer type 0041) containing melting point standards and using either the normal block lid (see El of Fig. 4) or a new type of vented block lid (supplied by the manufacturer) which reduces the effect of deposition of decomposition products on the lid upon the operation of the calorimeter. The vented block was obtained after the polyacrylonitrile studies were completed. A summary of the temperature scale calibrations and time constant studies using the dynamic method discussed in appendix C.3.4. is given in table $\mathrm{C}-6$ and the accompanying key. The vented block was involved in experiments having condition $B$ or $C . T_{\text {on }}(w)$, the onset temperature of melting, was determined as specified in fig. C-4 from a chart record with correction for the lag between the pens indicating 0 , the signal output, and $T_{p}$, the programmed temperature. A typical plot of $\mathrm{T}_{\text {on }}(\mathrm{w})$ versus $\mathrm{w}$ is given in $\mathrm{fig} . \mathrm{C}-5$; the estimated imprecision in $\mathrm{T}_{\text {on }}(0)$ is about $0.02 \mathrm{~K}$. Graphs of $\Delta_{\mathrm{T}}^{\mathrm{T}}$ STA are given in $\mathrm{fig} \cdot \mathrm{C}-6$. The $\mathrm{Pb}$ and $\mathrm{Zn}$ corrections, expt. $8 \mathrm{~b}$ and expt. 48 , are unrealiable and were not used. A better calibration curve than fig. C-6 is clearly desirable.

The difference in $T_{\text {on }}(0)$ between experiments $11 \mathrm{a}$ and $11 \mathrm{~b}$ and between $12 \mathrm{a}$ and $12 \mathrm{~b}$ are due to removing deposits of decomposition products of polyacrylonitrile on the normal block lid after experiment a of each pair of experiments. It is evident that $T_{\text {on }}(0)$ seems to be stable to $\pm 0.02 \mathrm{~K}$ for condition $\mathrm{A}$ so that the difference between condition $\mathrm{A}$ and $B$ of $0.1 \mathrm{~K}$ is real. The difference in $\tau_{0}$ due to the output filter of about 0.016 min (i.e. compare experiments $1,2 a$ with $11 a, 11 b$ and experiment 24 with 25) agrees with the longest time constant of the two pole

$$
\text { C.4-1 }
$$




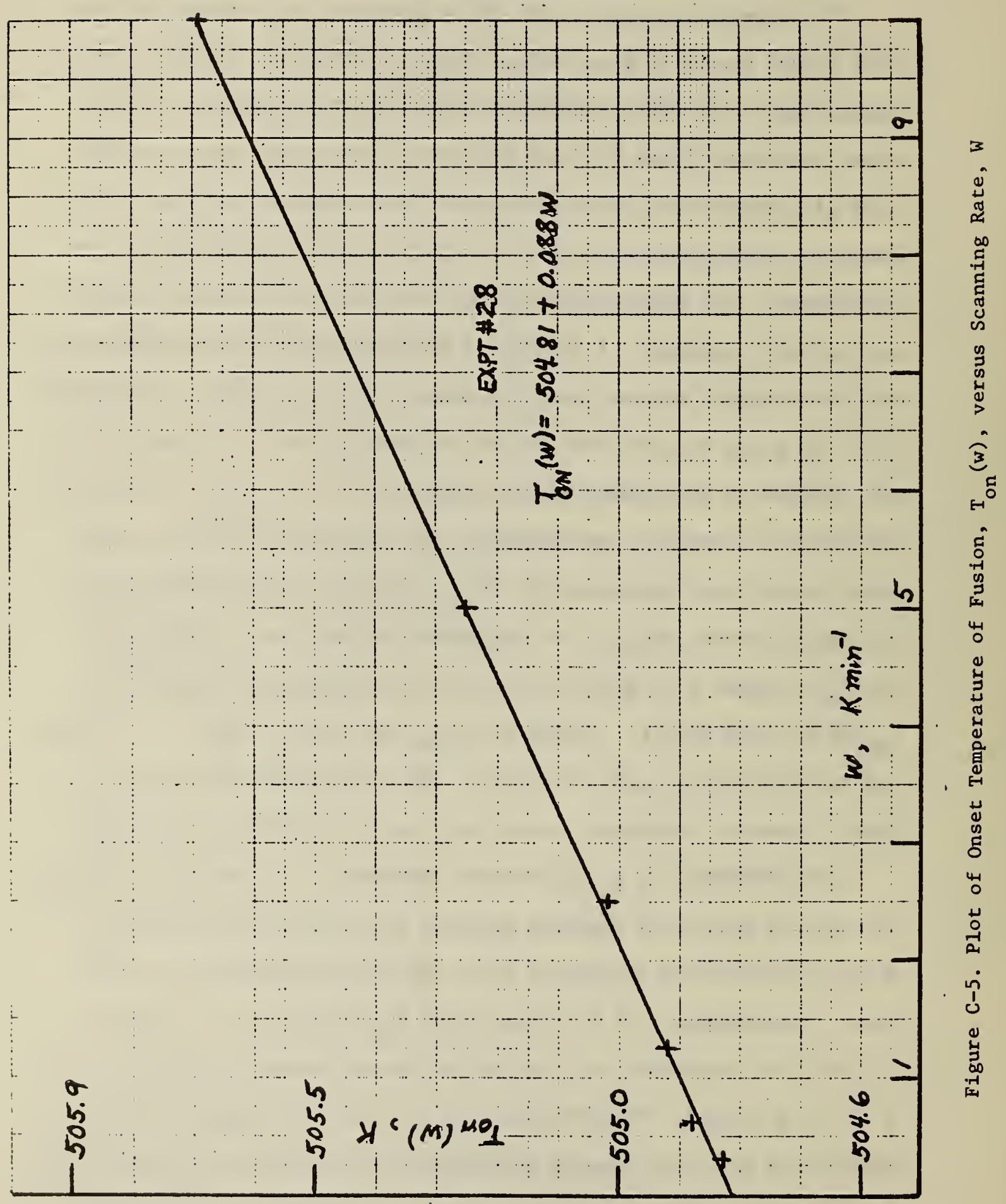

C. 4-2 


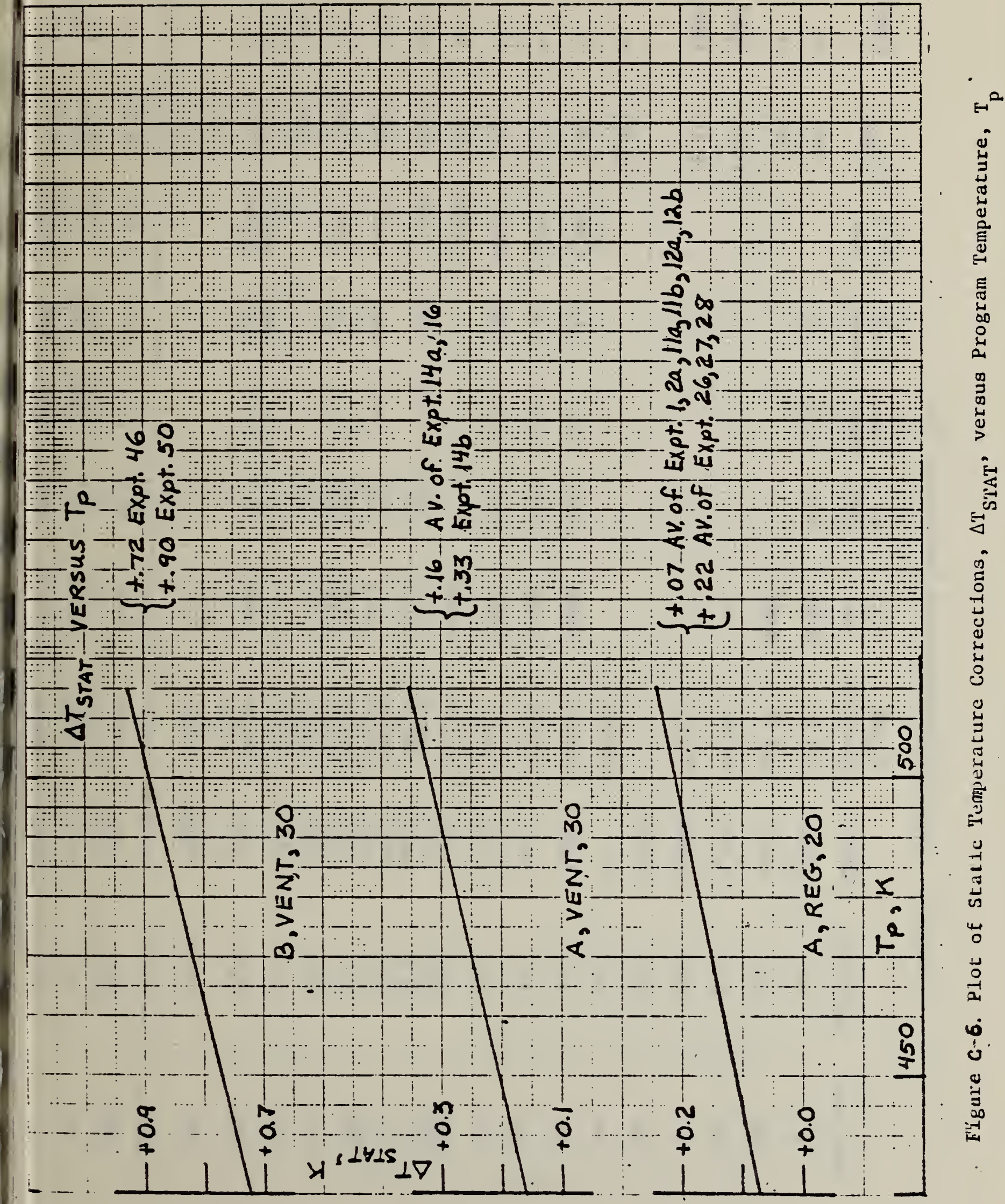




$$
\begin{aligned}
& \text { कั }
\end{aligned}
$$

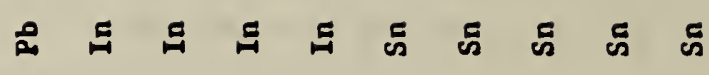

$$
\begin{aligned}
& \text { 동 } 5
\end{aligned}
$$

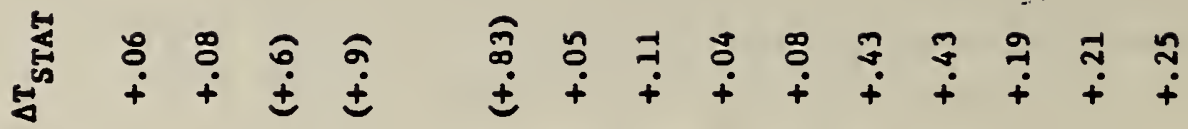

$$
\begin{aligned}
& \text { † 首 }
\end{aligned}
$$

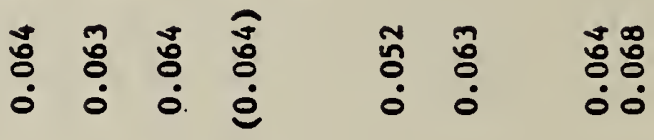

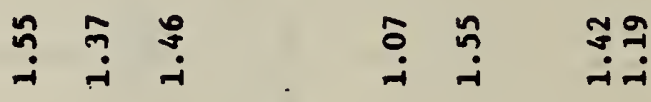

$$
\begin{aligned}
& \text { そै ๆี }
\end{aligned}
$$

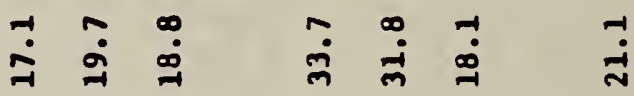

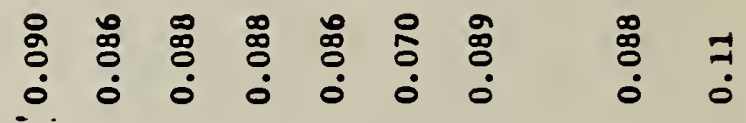

$$
\begin{aligned}
& \text { ஜ: }
\end{aligned}
$$

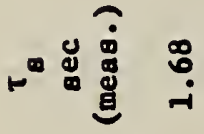

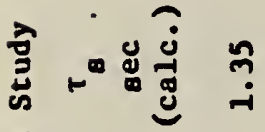

$$
\begin{aligned}
& =\underset{\text { 音 }}{\stackrel{0}{0}}
\end{aligned}
$$

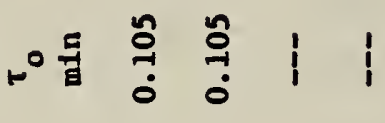

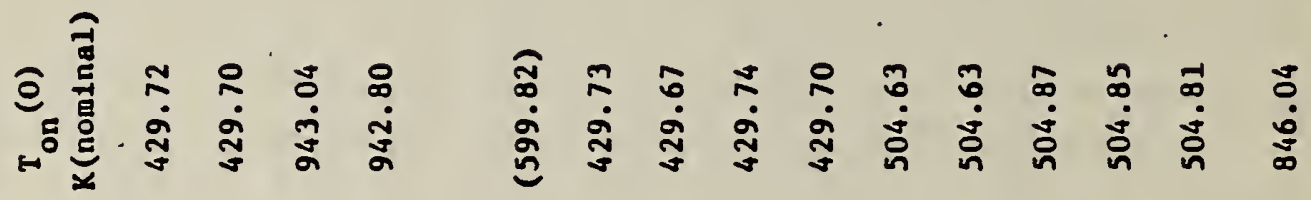

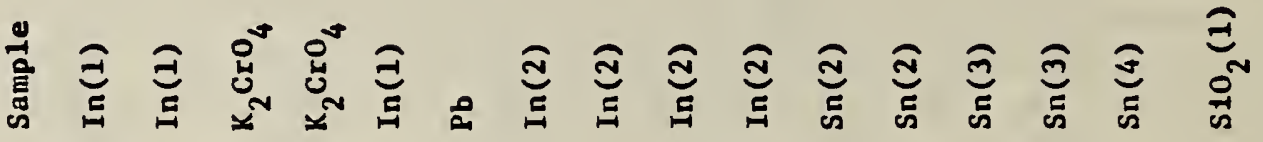

$$
\begin{aligned}
& \text { ఏ̊ }
\end{aligned}
$$

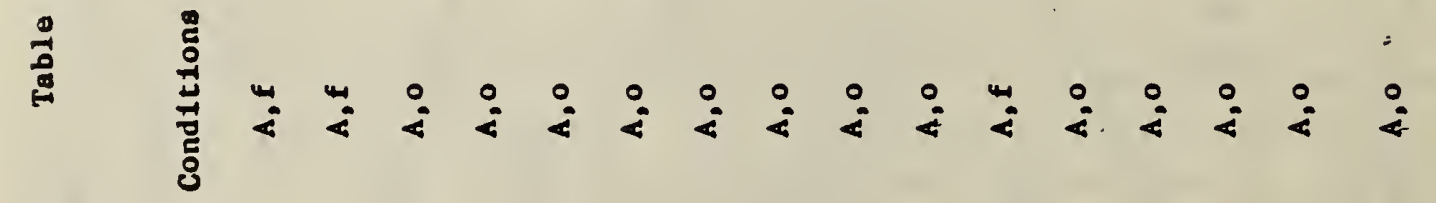

$$
\begin{aligned}
& \dot{g} \\
& \text { 渵 }
\end{aligned}
$$




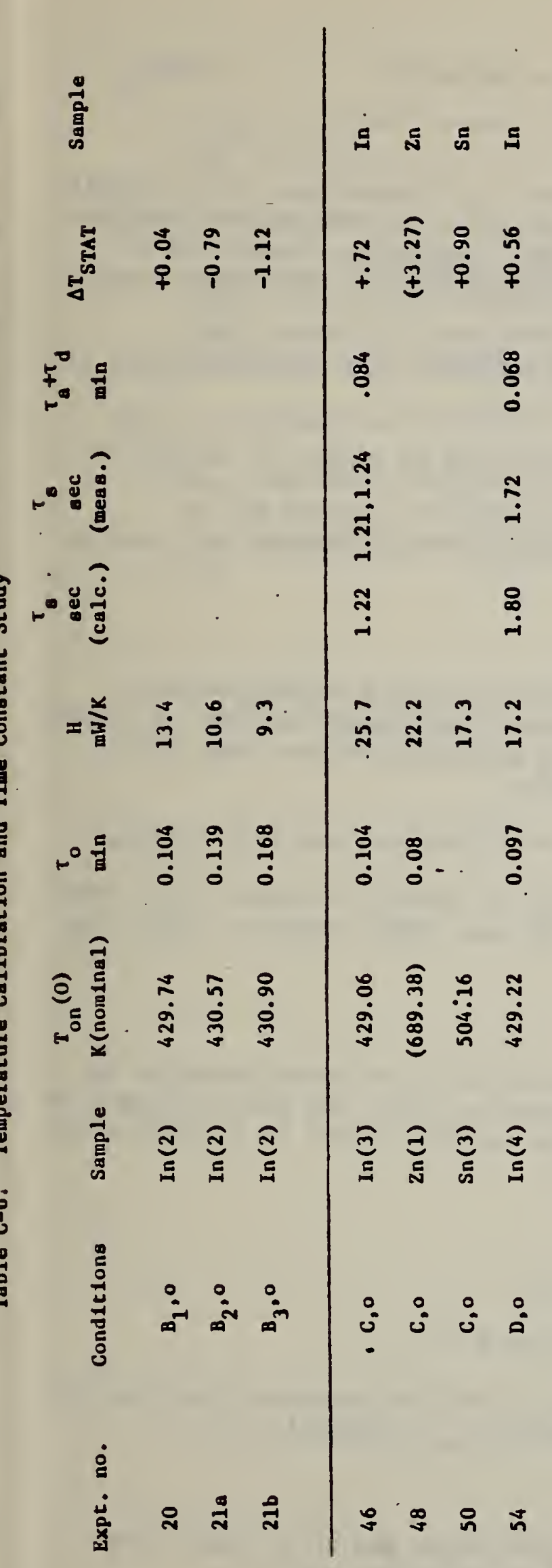




\section{Conditions}

A: Normal block lid, 20 psi (input) $\mathrm{N}_{2}$ flushing gas, solid sample holder cover and specific settings of the temperature calibration controls (temperature zero, temperature range, and $\Delta \mathrm{T}$ balance) determined procedures specified in instrument manual (supplied January (1977)).

o: Ordinate signal output filter bypassed (i.e. $\pm 50 \mathrm{mV}$ signal of section $9 \mathrm{H}$ of manual).

f: Ordinate signal filter in (i.e. $\pm 10 \mathrm{mV}$ signal of section $9 \mathrm{H}$ of manual).

B: Same as A except vented block lid, 30 psi (input) $\mathrm{N}_{2}$ flushing gas.

$\mathrm{B}_{1}, \mathrm{~B}_{2}, \mathrm{~B}_{3}:$

Same as B except the following are placed between melting point standard (in its container) and sample holder: B pyrex covers lip, $B_{2}$ inverted empty container bottom (type 0041), and $\mathrm{B}_{3}$ preceding plus coverslip.

C: Same as B but new temperature calibration controls settings.

D: Same as C except Indium sample in stated container (type 0062) as described in proposed ASTM test [155]; see also [194] fig. $2 b$ or [195], fig. 3 .

Sample formula (No.):

No. indicates various preparations of standards supplied by instrument manufacturer except for $\mathrm{Sn}(2)$ and $\mathrm{SiO}$ which are RM 758 supplied by NBS and ICTA and $\mathrm{Sn}(4)$ which is 0.999999 pure tin supplied by OSRM at NBS.

$T_{\text {on }}(0):$

$T_{\text {on }}(w)$ extrapolated to $w=0$.

$\tau_{0}:$ slope of a plot of $\mathrm{T}_{\text {on }}(\mathrm{w})$ versus $\mathrm{w}$.

$\mathrm{H}$ : $\mathrm{H}_{\mathrm{W}}$ is slope of leading edge of melting isotherm, see fig. $\mathrm{C}-4$, sêction C.3.4. H is nominally $h_{1 s}^{\prime}$ of appendix C.3.4. $\tau_{s}(\operatorname{calc}):$

$\left(C_{1}+C_{2}{ }^{f}\right) / H ; C_{1}$ is a measured value and $C_{2}^{f}$ is taken from [196]; see later summary of measurements of $C_{1}$. 


$$
\tau_{s} \text { (meas.) : }
$$

Slope of $\ln \left(0-0^{f}\right)$ versus time of decay curve (i.e. $A_{2}$, fig. C-4, section C.3.4.)

$$
\tau_{a}+\tau_{d}: \tau_{0}-\tau_{s} \text { (meas): }
$$

$\tau_{a} w$ is the lag of the apparent sample holder temperature behind the nominal program temperature, $T_{p} \cdot \tau_{d}$ is the longest time constant of instrument's holders, holder heaters, and difference power system (see Appendices B.3.3. and C.3.4.)

$$
\Delta \mathrm{T}_{\text {STAT }}: \mathrm{T} \text { (transition or fusion) }-\mathrm{T}_{\text {on }}(0) \text { : }
$$

The temperature correction added to $T$ to obtain the temperature of the sample container in the isothermal mode of operation of the instrument, see Appendix C.3.4. 
output filter ${ }^{+}$. The assumption that $\mathrm{H}$ is equal to $\mathrm{h}^{\prime}$ ss or, equivalently, that the sample and sample container temperatures are equal during the intilal stages of melting was checked by comparing the calculated value of $\tau_{s}, \tau_{s}($ cald.) (see key) with the time constant of the final part of decay curve of the melting endotherm, $\tau_{s}$ (meas.). These two time constants should be equal if $\mathrm{H}=\mathrm{h}_{1 \text { s }}^{\prime}$ as is discussed later in appendix C.4.2. With the exception of experiment 28 . (where $\tau_{s}$ (meas) decreases rather than increases as 0 approaches $0^{f}$ and experiment $14 \mathrm{~b}$, the agreement is reasonable. The value of $\tau_{a}+\tau_{d}$ seem roughly constant for a given condition ( $A, B, C$, or $D$ ) and nearly independent of $T_{p}$.

$\tau_{d}$ was measured by the analysis of a cooling curve produced by a partially supercooled exotherm of indium assuming the initial part of the exotherm is given by the dotted line of the insert in figure C-7 (see [191], section e). The initial part of observed exotherm does not fit the model as can be seen from figure $C-7$ but it seems reasonable to conclude that $\tau_{d}$ is $0.5 \mathrm{sec}$ or less. The resulting value of $\tau_{a}$ of about $0.06 \mathrm{~min}$, obtained by subtracting $\tau_{d}$ from $\tau_{a}+\tau_{d}$, is in agreement with that determined by Richardson, et al (see [188], fig. 1, the slope of their $\delta \tau_{0}$ line is $0.06 \mathrm{~min}$ ) using the heat capacity lag method summarized in Appendix C.3.5.

Some preliminary measurements aimed at determining the extent to. which the sample holder cup and lid depart from temperature uniformity were made in experiments 2C, 2la, and 21b (see key, table C-6a). The inverted uncrimpled sample container raises the Indium melting standard

\footnotetext{
the filtered output signal, 0 , is related to the filter input signal, 0 , by $\left(0.35 \sec ^{2} D^{2}+1.4 \sec D+1\right) 0=0^{\prime}$.
} 


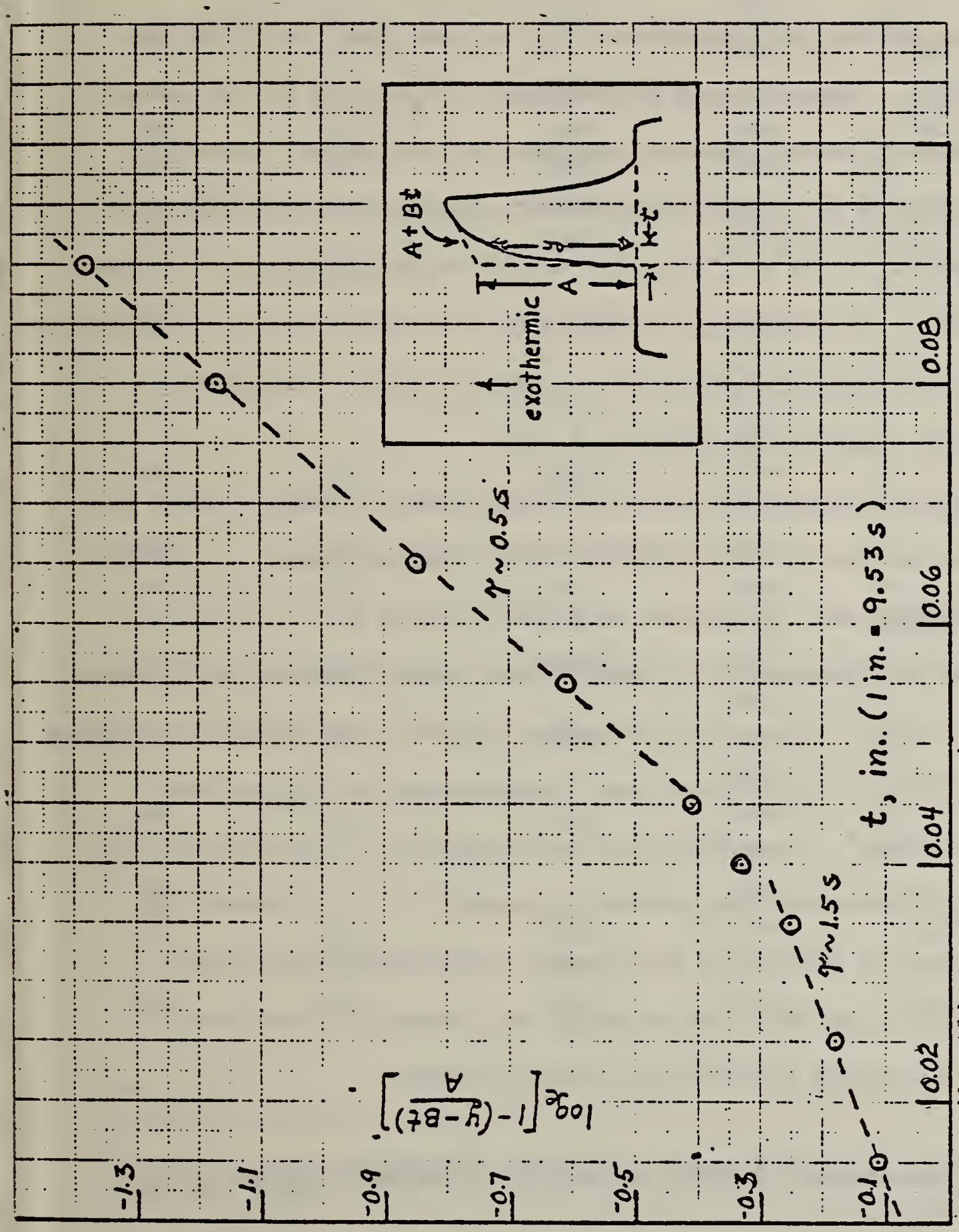


in its container to within about $0.03 \mathrm{~cm}$ of the sample holder lid. From the discussion of Appendix C.3.4., one should expect $\Delta T_{\text {STAT }}$ to decrease or $\mathrm{T}_{\text {on }}(0)$ to increase in proceeding from condition $\mathrm{B}$ or $\mathrm{B}_{1}$ to conditions $B_{2}, B_{3}$ if the holder lid temperature, $T_{\ell}$, is less than the holder cup temperature, $T_{s}$. These changes are observed. $T_{s}-T_{\ell} \gtrsim 1 \mathrm{~K}$. In spite of this the value of the calibration constant, $K$, for expts. $21 a$ and $21 b$ discussed below do not differ significantly from the other values of $K$ for conditions A, B, or C. Thus, for normal thermal geometry (i.e. conditions $A, B, C)$ it seems reasonable to conclude that effects of temperature gradients can certainly be taken in account by normal calibration procedures as was suggested in Appendix C.3.3.

A summary of energy scale calibrations using the single sample plus container run and base line analysis of ref [186] mentioned in Appendix C.3.5. for indium and tin fusions is given in table $c-7$. To confirm that $\mathrm{K}$ should be independent of temperature, a heat capacity calibration using SRM $720 \mathrm{Al}_{2} \mathrm{O}_{3}$ was carried out using the step jump procedure outlined in Appendix C.3.5. Initial and final temperatures are listed under "temperature range". No corrections were applied to these temperatures (1.e. $\Delta \mathrm{T}_{\mathrm{STAT}}$ corrections are assumed to cancel ${ }^{+}$). It is evident that the variation in $\mathrm{K}$ with $\mathrm{T}_{\mathrm{p}}$ is $1 \%$ or less. (Areas were calculated numerically from the chart record using the trapezoidal rule with an uncertainty estimated to be of the order of $0.5 \%$ ).

\footnotetext{
The uncertainty caused by this assumption is estimated to be of the order of $0.5 \%$.
} 
Table C-7 Energy Calibration

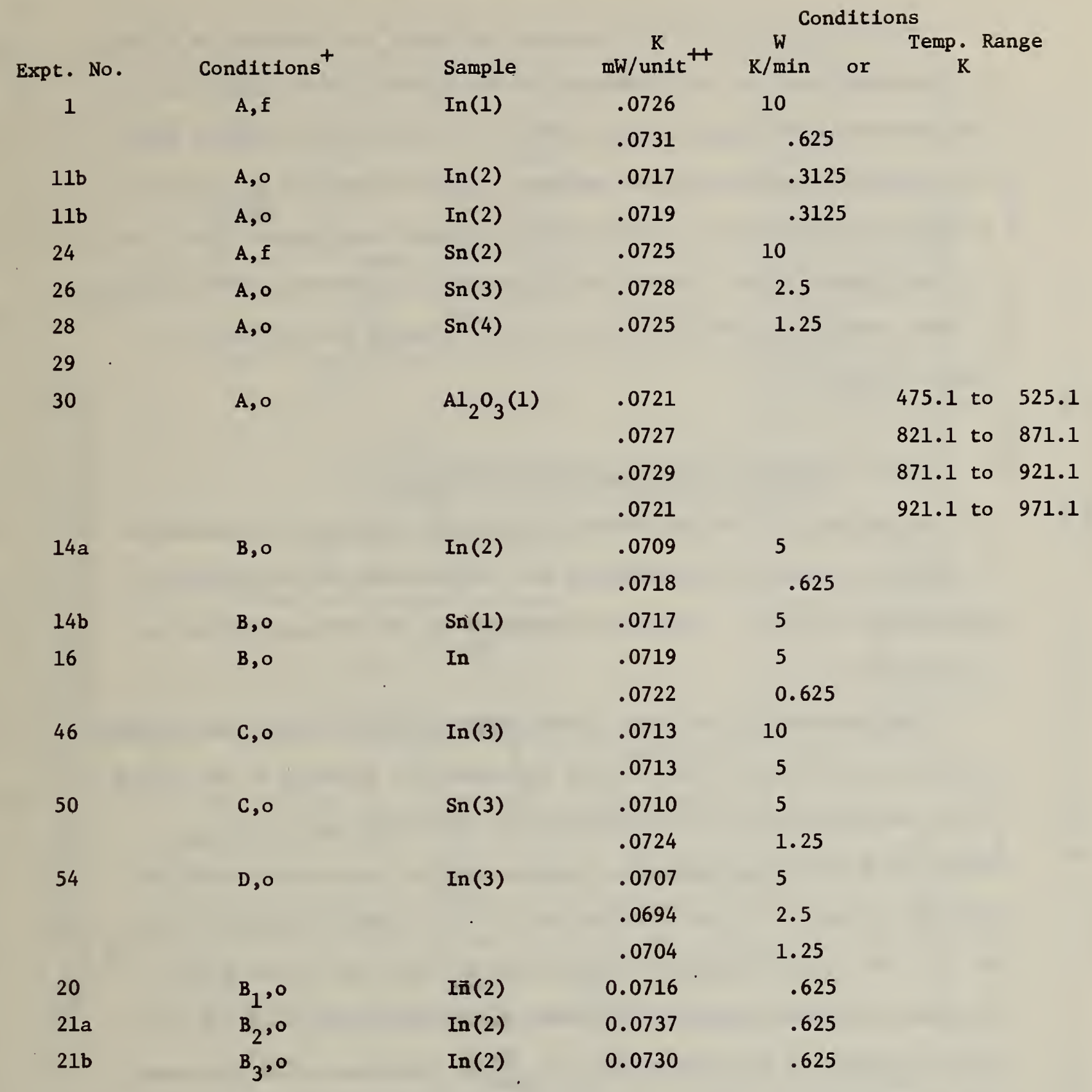

\footnotetext{
Tee key to table 24 .

${ }^{+} 1$ unit on chart recorder when setting of instrument is " $1 \mathrm{mcal} / \mathrm{sec}$, chart $0.1 \mathrm{mV}$ full scale.
} 
The heat capacity of the aluminum containers was measured as a part of two experiments on the decomposition of nitrocellulose using the dynamic method described in ref. [199], p. 17, with the exception that a heat capacity standard run was omitted. (w was assumed to be exact and an average $\mathrm{K}$ from table $\mathrm{C}-6$ was used). Although experimental conditions in experiments 14 and 16 were far from optimal, reasonably good agreement of both experiments with the data for pure aluminum was obtained as shown in $\mathrm{fig} . \mathrm{C}-8$.

\section{C.4.2. Analysis of the Calibration Procedures}

An analysis of the performance of the DSC apparatus was undertaken to provide a basis for interpreting the calibrations and decomposition experimental results. This is an extension of the treatment given in Appendix C.3.

The operation of the input temperature detection circuit was analyzed. Calculations indicated that, if the instrument is adjusted at the In and $\mathrm{K}_{2} \mathrm{CrO}_{4}$ melting points as specified in the instrument manual, $\Delta \mathrm{T}_{\text {STAT }}$ should have the form of smooth, roughly paroblic, curve with values of zero at 429 and $943 \mathrm{~K}$ and a maximum of 1 or $2 \mathrm{~K}$ midway between 429 and $943 \mathrm{~K}$. The qualifications on this result are that the In and $\mathrm{K}_{2} \mathrm{CrO}_{4}$ melting points are assumed to be known on the IPTS scale to $0.1 \mathrm{~K}$ and the instrument is adjusted so that $\Delta \mathrm{T}_{\text {STAT }}$ is less than $0.1 \mathrm{~K}$, at these two melting points. In the calculations, the resistance-temperature characteristics of the platinum resistance thermometers were assumed to be those for strain-free platinum and the measured programmed variation in the supply voltage of our DSC was used. The results of experiments $8 \mathrm{~b}(\mathrm{~Pb})$ and $50(\mathrm{Zn})$ of table C-6 were not included in Fig. C-6 because 


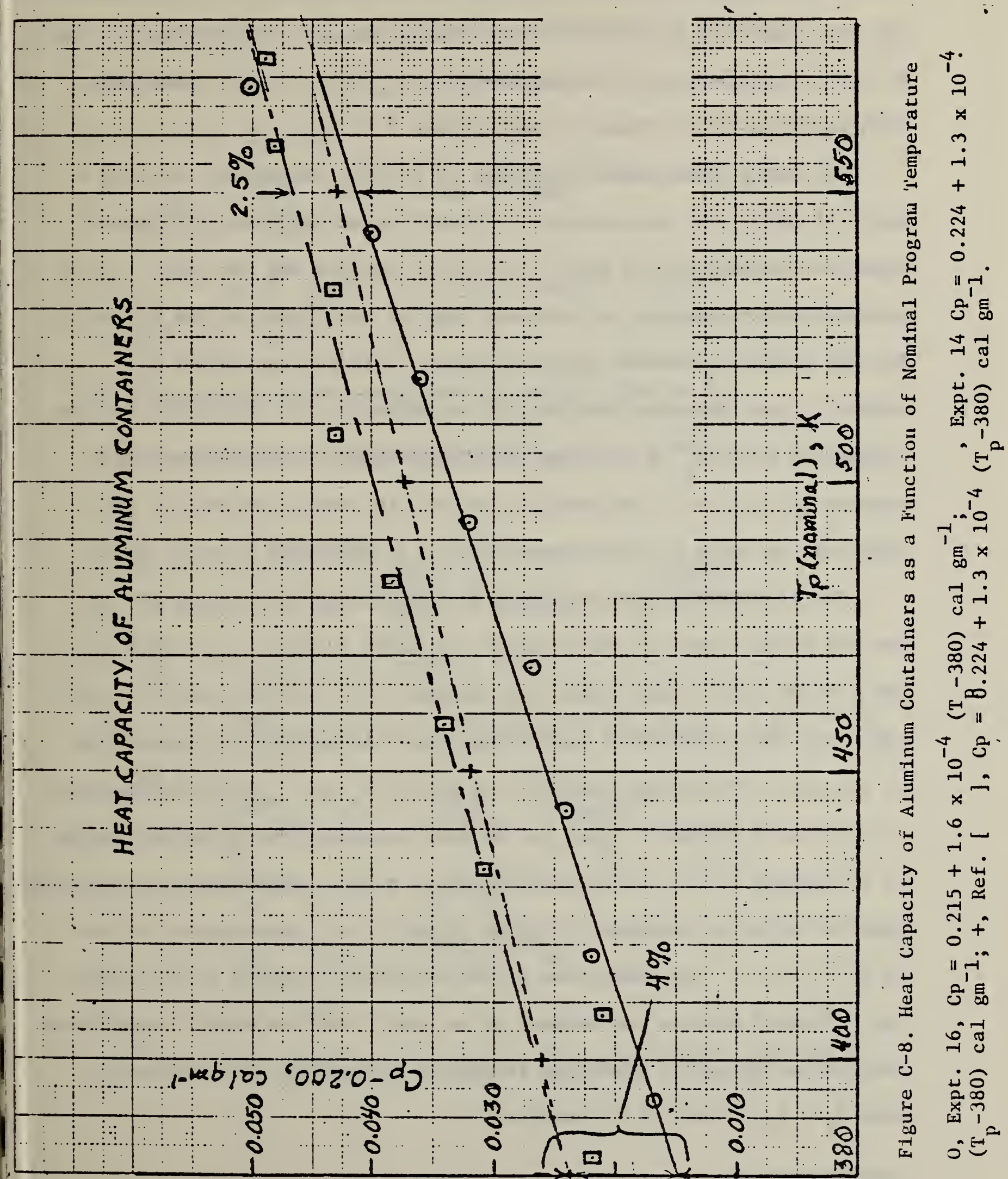


of the abrupt shift that they would be caused in the plot of $\triangle T_{\text {STAT }}$ versus $T_{P}\left(\Delta T_{S T A T}\right.$ for the tin melting point is reliable). This decision was also supported by indications of premelting due to impurities in the $\mathrm{Pb}$ and $\mathrm{Zn}$ endotherms and the agreement of $\mathrm{T}_{\text {on }}(0)$ for $\mathrm{SiO}_{2}$, expt. 29, with measurements of others to better than $1 \mathrm{~K}$.

The energy measurement equations of the DSC instrument, entries 4 and 5 of table $\mathrm{C}-5$, were solved to obtain the approximate differential equations satisfied by $S$ and $\left(T_{S}+T_{I}\right) / 2$. The aim was two fold: (1) to provide apriori support for and some idea of the limits of the eq. C-17a for the ordinate transfer function and eq. C-18 for the lag of $\mathrm{T}_{\mathrm{s}}$ behind $T_{p}$ in a scanning run, and (2) to determine the conditions for the assumption that $\mathrm{DT}_{S}^{+}$is the same for a measurement and blank run which reduces to $\mathrm{DT}_{S}=\mathrm{w}$. This equality for $\mathrm{DT}$ is used to derive all the equations in table $\mathrm{C}-4$ and appendices C.3.4. and C.3.5.

The assumptions used to simplify entries 4 and 5 of table C-5 for the initial part of the analysis are given in entry 1 of table $\mathrm{C}-8 \mathrm{a}$. The lid and cup of each holder are assumed to be uniform, entry la, and the total heat capacity of each holder plus its contents is assumed to be the same. Following $O^{\prime}$ Neill et al [176], $\ddot{Q}_{S}$ and $\ddot{Q}_{r}$ were approximated by entry lc. $\dot{Q}_{j}(0), j=s, r$, is the rate of absorption of either holder at a constant initial temperature $T_{j}(0), j=s, r$. Experimental measurements show the error in assuming $\dot{Q}$ varies linearly with temperature is about $2 \%$ if $\theta \leq 25 \mathrm{~K}$. The resistance of the electrical heaters of the sample and reference holders are assumed to be equal, and the manual temperature calibration procedure discussed previously is assumed to, in effect, make $\mathrm{f}_{\mathrm{a}}=\mathrm{f}_{\mathrm{p}}$ of entry 1 of table $\mathrm{C}-5$.

$t_{D}=d / d t$ 
Table C-8a. Approximate Equations for DSC, Formulation of Equations

1. Assumptions
a) $T_{\ell}=T_{S}, T_{m}=T_{r}$.
b) $\quad \sum_{j} C_{j}=\sum_{j, l, 1,2} C_{j}=C ; C$ indep. of temp.
c) $\ddot{Q}_{j}=\dot{Q}_{j}(0)-h_{0} \theta_{j} ; j=s, r ; h_{0}$ indep. of temp.
$\theta_{j}=T_{j}-T_{j}(0)$

d) electrical heater resistance of each holder is $R_{H}$.

e) temperature calibration adjusts $f_{p}=f_{a}, T_{0}=0$

\section{Energy Balance Equations}
a) $t=0: \quad 0=\ddot{Q}_{j}^{\prime}(0)+\frac{V_{A}(0)^{2}}{2 R H}+1 / 2 I_{0}^{2} R_{H}+N_{j} \frac{I_{0} \Delta V(0)}{2} ; j=s, r$
$N_{j}=-1, j=s ; N_{j}=+1, j=r$
b) $t>0: \operatorname{cD} \theta_{j}=-h_{0} \theta_{j}+k_{a}\left(Y-\theta_{s}-\theta_{r}\right)+N_{j} k_{j d}\left(\theta_{s}-\theta_{r}\right)+p(t)+W_{j}$;
$j=s, r$
c) $k_{a}=\frac{V_{A}(0)}{2 R_{H}} G_{a} f ; k_{j d}=\frac{I_{0} G_{d} f_{d}}{2}-\frac{\Delta V(0)}{4 R_{H}} G_{d} f_{d} N_{j} ; j=s, r$
d) $p(t)=k_{a}^{\prime}\left(Y-\theta_{s}-\theta_{r}\right)^{2}+k_{d}^{\prime}\left(\theta_{s}-\theta_{r}\right)^{2}$
e) $k_{a}^{\prime}=\left(G_{a} f_{a}\right)^{2} / 8 R_{H} ; k_{d}^{\prime}=\left(G_{d} f_{d}\right)^{2} / 8 R_{H}$
f) $Y=2\left[T_{p}-T_{p}(0)\right]$
g) $\mathrm{W}_{s}=\mathrm{P}_{2}-\mathrm{C}_{1}\left(\mathrm{DT} \mathrm{T}_{1}-\mathrm{DT} \mathrm{T}_{\mathrm{s}}\right)-\mathrm{C}_{2} \mathrm{DT} \mathrm{T}_{2}-\mathrm{C}_{2}^{1} \mathrm{DT}_{\mathrm{s}} ; \mathrm{W}_{\mathrm{r}}=-\sum_{i} \mathrm{C}_{i}\left(\mathrm{DT} \mathrm{T}_{i}-\mathrm{DT} \mathrm{T}_{\mathrm{r}}\right)$ 
Table C-8b. Approximate Equations for DSC, Solution of Entry 2, Table C-8a

1. Equation for $S-S(0)=\Delta S ; \Delta S=\left[k_{s d}+k_{r d}\right]\left[\theta_{S}-\theta_{r}\right] f_{\Delta V} / I_{0}$
a) $\left(\tau_{a}^{\prime} D+1\right)\left(\tau_{d}^{\prime} D+1\right) K \Delta S=\left(1+\tau_{a}^{\prime} D\right)\left(W_{s}-W_{r}\right)$
b) $\tau_{a}^{\prime}=\frac{c}{2 k_{a}\left(1+r_{a}\right)} ; r_{a}=\frac{h_{0}}{k_{a}}$
c) $\tau_{d}^{\prime}=\frac{c}{2 k_{d}\left(1+r_{d}\right)} ; r_{d}=\frac{h_{0}}{k_{a}} ; k_{d}=\left(k_{s d} k_{r d}\right) / 2$
d) $K=\frac{k_{s d}+k_{r d}}{f_{\Delta V}^{G} d^{f} d}\left(1+r_{d}\right)=\frac{I_{0}}{f_{\Delta V}}\left(1+r_{d}\right)$;

2. Equation for $\left(\theta_{s}+\theta_{r}\right)$ when $r_{a} \ll 1$
a) $\left(\tau_{a}^{\prime} D+1\right)\left(\tau_{d}^{\prime} D+1\right)\left(\theta_{s}+\theta_{r}\right)=\tau_{a}^{\prime}\left(1+\tau_{d}^{\prime} D\right)\left(w_{s}+w_{r}\right) / C+$

$$
\left(1+\tau_{d}^{\prime} D\right) Y+\left(1+\tau_{d}^{\prime} D\right)\left[\frac{k_{a}^{\prime}}{k_{a}}\left(Y-\theta_{s}-\theta_{r}\right)^{2}+\frac{k_{d}^{\prime}}{k_{a}}\left(\theta_{s}-\theta_{r}\right)^{2}\right]
$$

b) Rearrangement of $2 a$ when $r_{d} \ll 1$ :

$$
\begin{aligned}
& \left.\left\{\tau_{a}^{\prime} \tau_{d}^{\prime} D+\left[\tau_{a}^{\prime}+\tau_{d}^{\prime}\left(1+\frac{\Delta V_{A}}{V_{a}(0)}\right)\right] D+\left[1+1 / 2 \frac{\Delta \nabla_{A}}{V_{A}(0)}\right]\right)\right\}\left(Y-\theta_{s}-\theta_{r}\right)= \\
& +\tau_{a}^{\prime}\left(1+\tau_{d}^{\prime} D\right)\left(D Y-\frac{2 W_{r}}{C}\right)-\frac{1}{2 k_{a}}\left(1+\tau_{d}^{\prime} D\right)\left\{K \Delta S\left[1+\frac{K \Delta S}{4 R_{H} I_{0}^{2}}\right]+\right. \\
& \left.\tau_{d}^{\prime} D K \Delta S\right\} ; \\
& \Delta V_{A}=V_{A}-V_{A}(0)=k_{a} f_{a}\left(Y-\theta_{s}-\theta_{r}\right) / 2 .
\end{aligned}
$$


The static energy balance for the sample and reference holders, at time $t=0$, entry $2 a$, were subtracted from entries 4 and 5 of table $C-5$ to give a dynamic energy balance equation for each holder at time $t>0$, entry $2 b$. In entry $2 a, V_{A}(0)$ and $\Delta V(0)$ are the static output voltages of the average and difference amplifiers. The parameters $R_{H}, I_{0}, G_{a}$, $G_{d}, f_{a}$ and $f_{d}$ are defined in table $C-5$. The second through fourth terms on the right side of entry $2 \mathrm{~b}$ are the dynamic terms contributed by $\mathrm{P}_{\mathrm{A}}+$ $P_{B} \pm 1 / 2 P_{D}$ of entries 4 and 5 of table $C-5 . \quad-W_{S}$ and $-W_{r}$ are the rates of increase of the enthalpy of sample and reference containers and their contents minus the corresponding rates of increase assuming their temperatures equal $\mathrm{T}_{\mathbf{S}}$ and the heat capacity of the sample does not change. (The value of $\theta_{j}$ is assumed to be small enough that $C_{s}, C_{l}, C_{1}, C_{r}, C_{m}$, $\mathrm{C}_{3}$, and $\mathrm{C}_{4}$ are constant.)

Using operator algebra, entry $2 b$ of table $C-8 a$ can be rearranged to give entries $1 a$ and $2 a$ of table $C-8 b$. S is the ordinate output of the instrument if the longest time constant of the difference amplifier, $\tau_{d}^{\prime \prime}$, is zero. $S(0)$ is the value of $S$ at $t=0$. The relation between $S$ and $\theta_{s}-\theta_{r}$ follows from entries 2 and 6 of table $C-5$ and entry $2 c$ of table C-8a. Entry la of table C-8b corresponds to the DSC energy . measurement equation given in entry 6 of table C-5. Entry la indicates the ordinate transfer function is given by

$$
S=\left(\tau_{d}^{\prime \prime} D+1\right)\left(\tau_{d}^{\prime} D+1\right) 0 \sim\left[1+\left(\tau_{d}^{\prime}+\tau_{d}^{\prime \prime}\right) D\right] 0
$$

Entry $2 a$ is the expression for the average temperature of the sample and reference holders assuming $r_{a} \ll 1$. Using the definitions of $k_{a}^{\prime}, k_{a}, k_{d}^{\prime}$ and $k_{d}$ of table $C-8 a$, entry $2 a$ of table $C-8 b$ can be rearranged 
for $r_{d} \ll 1$ to give entry $2 b$. The nonlinear entries on the left side can be dropped since experimental measurements show $\Delta V_{A} / V_{A}(0)$ is of the order of .02 for $\theta \leq 25 \mathrm{k}$. The nonlinear term in $\Delta S$ can be dropped if $\mathrm{K} \Delta \mathrm{S} / 8$ is much less than the static power supplied by the constant current generator of fig. C-3, which is usually the case (e.g. at $355 \mathrm{~K}, 1 / 2$ $I_{0}{ }^{2} R_{\mathrm{H}}$ is $\sim 80 \mathrm{~mW}$ so $\mathrm{K} \Delta \mathrm{S}$ must be much less than $0.64 \mathrm{~W}$ ). Dropping the nonlinear terms from entry $2 b$, one has the result that $T_{p}$ leads ${ } T_{s}+$. $\mathrm{T}_{\mathrm{r}}$ )/2 by $\tau_{\mathrm{a}}^{\prime} \mathrm{w}+\Delta \mathrm{T}_{\mathrm{STAT}}$. After starting transients have decayed out and no thermal event is occuring in a scanning run (i.e. $\Delta S$ and $W_{r}$ are zero). In the DSC-2 an additional increment of constant power that is proportional to $w$ is supplied to each holder to null out the $\tau_{a}^{\prime}$ w temperature $\log$. Also one has the result that the absolute fractional error, $\left|D T_{s}-W\right| / W$ is less than $|D K \Delta S| / 4 \mathrm{kaW}^{+}$. The error is small because $\mathrm{k}_{\mathrm{a}}$ is large as O'Neill [176] has pointed out.

If $h_{0}, C$, and $R_{H}$ of the sample calorimeter exceed the corresponding quantities of the reference calorimeter by the fractional amounts of $\delta_{0}$, $\delta_{C}$, and $\delta_{R} / 2$, the following terms must be added to the right side of entry $1 \mathrm{~b}$ of table $\mathrm{C}-8 \mathrm{~b}$ if $\mathrm{r}_{\mathrm{a}}=\mathrm{r}_{\mathrm{d}}=0$

$$
\begin{gathered}
-\left[h_{0}\left(\delta_{R}+\delta_{0}\right)+c\left(\delta_{R}+\delta_{c}\right) D\right] Y / 2 \\
-\left[\tau_{a}^{\prime}\left(\delta_{R}+\delta_{c}\right) D\right]\left[k_{d}^{\prime}\left(\theta_{s}-\theta_{r}\right)^{2}+k_{a}^{\prime}\left(Y-\theta_{s}-\theta_{r}\right)^{2}\right.
\end{gathered}
$$

$\mathrm{K}$ is multiplied by $1-\delta_{\mathrm{R}} / 2$ and both $\tau_{\mathrm{a}}^{\prime}$ and $\tau_{\mathrm{d}}^{\prime}$ are multiplied by $1+\left(\delta_{\mathrm{c}}+\right.$ $\left.\delta_{R}\right) / 2$ if $\delta_{C}$ and $\delta_{R}$ are small. The first line of the added terms contributes

The equivalent condition for the QDTA instrument is $|\mathrm{DKS}| / \mathrm{h}_{\mathrm{rb}} \mathrm{w}$. 
a constant term of $\delta_{c} \mathrm{Cw}$ due to the mismatch in heat capacities and a term proportional to wt due to the mismatch in the dynamic rate of heat loss and heater resistances of both calorimeters. These terms support the idea that the linear extrapolation of base lines as shown in fig. C-4 correctly accounts for heat loss mismatch. The $\delta_{c} C_{w}$ term is what one should expect and explains why the time constant for $\delta_{c} C_{w}$ to appear in the ordinate signal is nearer to $\tau_{a}$ than $\tau_{d}$. Using an argument similar to that used to show that the nonlinear terms are small in entry $2 b$ of table $C-8 b$, one can show that nonlinear terms in the second line of added terms are smaller than those of entry $2 b$, table $c-8 b$, by a factor of $\delta_{r}+\delta_{c}$ and hence can ordinarily be dropped.

It should be pointed out that if the temperature of the holder cups is, as we showed, essentialiy independent of $W_{S}$ the effect of the lid can be accounted for by adding the analogous terms into $\mathrm{W}_{\mathrm{S}}$. The assumption that the sample holder $l i d \mathrm{dT} / \mathrm{dt}=\mathrm{w}$, which is basic to the measurement equaticns, can be solved and compared with suitable experiments.

This has not been done as of yet.

The major drawback to the model is that the time constants of the electrical heaters and resistance thermometers of the holders have not been explicitly taken into account. Physically, one can see that they are in fact the main components of $\tau_{a}$ and $\tau_{d}$. Introduction of the appropriate equations for these time constants produces a $6^{\circ} \times 6_{\text {matrix }}$ that has not been reduced to a form whose meaning is simple to analyze in physical terms. After this is done the limitations of the preceding or zero th order model of the DSC will then be known in a experimentally measurable form. 
The form of $W_{S}(t)$ for a melting endotherm is of interest for calibration of the temperature scale, determination of ' ${ }^{\prime}{ }_{1 s}$ ', and evaluation of time constants. If the restriction that the temperatures of the sample and sample container be equal is removed it can be shown that $\mathrm{W}_{\mathbf{S}}$ of entry $2 a$ and $2 b$ of table $C-8 b$ is

$$
W_{s}(t)=w^{f}\left(t-t_{m}\right)-w A\left(I-\exp \left[-\left(t-t_{m}\right) / \tau_{I}\right]\right) ; t>t_{m} \quad C-24 a
$$

where

$$
\begin{aligned}
& \mathrm{H}^{\mathrm{f}}=\frac{\mathrm{h}_{12^{\mathrm{h}} \mathrm{h}_{1 \mathrm{~s}}^{\prime}}^{\prime}}{\mathrm{h}_{12}^{\mathrm{f}}+\mathrm{h}_{1 \mathrm{~s}}^{\prime}} \\
& A=\left[C_{1} r^{f}+C_{2}^{i}\left(r^{f}-r^{i}\right)\left(1+r^{f}\right] /\left[\left(1+r^{f}\right)^{2}\right]\right. \\
& r^{f}=\frac{h_{1 s}^{\prime}}{h_{12}^{f}} ; r^{i}=\frac{h_{1 s}^{\prime}}{h_{12}^{i}} ; \tau_{1}=\frac{c_{1}}{h_{1 s}^{\prime}+h_{12}^{f}}
\end{aligned}
$$

The superscript $i$ and $f$ refer to before and after melting, respectively. $t_{m}$ is the time of melting (i.e. ${ }^{t} t_{m}$ apart from a constant is the program temperature at the melting point). The remaining symbols are as defined in Appendix C.3. One can see from eq. C-24b that $H_{f}$ is equal to $h_{1 s}^{\prime}$ only if $r^{f}$ of eq. C-24d is negligible. From the analysis of samplelimited melting given by $0^{\prime} \mathrm{Neill}$ [176], one concludes on physical grounds that for pure metals the melting point endotherm, $W_{S}$ should become progressively more concave down as melting proceeds. $\mathrm{wH}^{\mathrm{f}}$ is the initial slope as $t-t_{m}$ becomes small but still is greater than $\tau_{1}$ of eq. C-24d (if A is not negligible). For a pure substance there should be a. relatively sharp break in the output signal upon melting. $\quad\left(W_{S}=0\right.$ before melting and is given by eq. C-24a afterwards). Since $\tau_{d}$ is small any deviation from this behavior should be due to impurities as Flynn has implied [190]. 
This was verified by observation of a sharp break (i.e. curvature of break is consistent with $\tau_{d}<0.5 \mathrm{sec}$ ) in the output endotherm for very pure tin in experiment 28 of table 11.

The values of $\tau_{s}$ (calc.) and $\tau_{s}$ (meas) of columns 7 and 8 of table $C-6$, whlle agreeing within experimental uncertainty, should obey eq. C-25 if the melted sample and the sample container have different but uniform temperatures. One can show that $\tau_{s}$ (meas) and $\tau_{s}$ (calc) "box" the value of $\tau_{s}$, $\left(c_{1}+c_{2}^{f}\right) / h_{1 s}^{\prime}$, that prevails if the temperature of sample and sample container are uniform.

$$
\tau_{s} \text { (meas) }<\frac{c_{1}+c_{2}^{f}}{h_{1 s}^{\prime}}<\frac{\left(c_{1}+c_{2}^{f}\right)\left(1+r^{f}\right)}{h_{1 s}^{\prime}}=\tau_{s} \text { (calc) }
$$

If $\tau_{s}$ (meas) $=\tau_{s}$ (cald), then $r^{f}=0, H=h_{1 s}^{\prime}$, and the temperature of the sample after it starts melting and the temperature of the sample container are equal. It is evident from table C-6 that, experimentally, $\tau_{s}$ (cald) is nearly equal but slightly greater than $\tau_{s}$ (meas). One possible explanation is that the effect of $\tau_{d}$ is influencing the decay curve (i.e., the assumption that it does not nay be in error). In any event, it seems reasonable that $\mathrm{r}^{\mathrm{f}}$ is evidently negligible and $\mathrm{H}=\mathrm{h}_{1 \mathrm{~s}}^{\prime}$ as was mentioned earlier. 


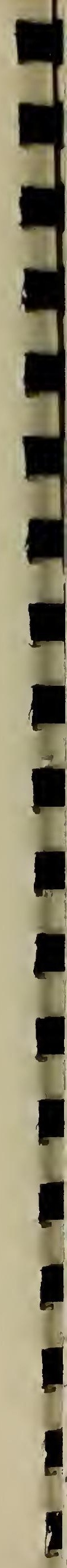


C.5. Error Analysis for Four Assumptions

In this appendix we consider four assumptions of the ASTM method:

(1) that the value of $g\left(\eta_{d}\right)$ at the temperature, $T_{d}$, of maximum rate is known and does not vary with the nominal scanning rate, w, (2) that

deviations of the rate of change of temperature of the sample from $w$ does not cause $g\left(\eta_{d}\right)$ to vary with $w$ or the temperature of the sample at maximum rate, (3) that maximum in the observed ordinate signal corresponds to the maximum in the rate of decomposition of sample, and (4) the rate of change of the sample temperature at $T_{d}$ equals $w$. The third and fourth assumptions are assumptions 1 and 2 of table 11 of the text and are discussed in the final section of this Appendix.

\section{C.5.1. The Value of $g\left(n_{d}\right)$ at the Maximum $\mathrm{d} n / \mathrm{dt}$}

The basic kinetic equation is

$$
\mathrm{dn} / \mathrm{dt}=f(n) \mathrm{k}(\mathrm{T})=f(\eta) \mathrm{A} \exp (-E / R T)
$$

The derivative with respect to time is set equal to zero at the maximum:

$$
d^{2} \eta / d t^{2}=0=(d f / d \eta)_{d}(d n / d t)_{d} k\left(T_{d}\right)+f(d k / d T)_{d}(d T / d t)_{d}
$$

Substitution of $w$ for $d T / d t$, and the expressions for $k(T)$ and $d n / d t y i e l d s$

$$
g\left(\eta_{d}\right) \equiv-(d f(n) / d \eta)_{d}=(w E / R A) T_{d}{ }^{-2} \exp \left(E / R T_{d}\right) \quad \ldots C-26
$$

The method assumes $w, E$ and $A$ are constant and $g\left(\eta_{d}\right)=1$ when $A$ is calculated using equation $\mathrm{C}-26$.

For the general rate law for simple reactions,

$$
f(n)=(1-n)^{n}
$$

$g\left(n_{d}\right)=1$ for first order reactions, by definition. For other values of $n$, $g\left(n_{d}\right)$ is nearly unity and almost independent of $T_{d}$. This is shown below. Later the question of other rate laws is considered. 
To show the approximation equation 11 is integrated to obtain $G(n)$ a readily calculable function and then the product $g\left(n_{d}\right) G\left(n_{d}\right)$ is reduced to an approximate numerical form.

$$
G(n) \equiv \int_{0}^{n} d \eta / f(n)=\int_{T_{0}}^{T} \frac{k_{T} d T}{d T / d t}=(A / w) \int_{T_{0}}^{T} \exp (-E / R T) d T \quad C-27 a
$$

where $G$ can be written as

$$
G(n)=(A E / w R)\left[P(X)-P\left(X_{0}\right)\right]
$$

w1th

$$
P(X) \equiv-\int_{\infty}^{X} x^{-2} \exp (-X) d X
$$

and

$$
X=E / R T ; X_{0}=E / R T_{0}
$$

An appropriate assymptotic expression for $P(X)$ is [161]

$$
P(x)=x^{-2} \exp (-x)\left[1-2 / x+6 / x^{2} \ldots \ldots\right]
$$

Combining $C-26$ and $C-27 b$, with $T_{c}$ chosen to make $P\left(X_{0}\right)$ negligible gives

$$
g\left(n_{d}\right) G\left(n_{d}\right)=x_{d}^{2} \exp \left(x_{d}\right) P\left(x_{d}\right)=1-2 / x_{d}+6 / x_{d}^{2}
$$

The product gG is evaluated using equation 8 to yield

$$
\begin{aligned}
g\left(n_{d}\right) G\left(n_{d}\right) & =\left(g\left(n_{d}\right)-n\right) /(1-n) ; n \neq 1 \\
& -\ln \left(1-n_{d}\right) ; n=1
\end{aligned}
$$

Thus, from equations $\mathrm{C}-29$ and $\mathrm{C}-30$

$$
g\left(n_{Q}\right) ; n=1
$$

where

$$
\begin{gathered}
\emptyset=2(1-n)\left(1 / x_{d}-3 / x_{d}^{2}+\ldots\right) ; n \neq 1 \\
\emptyset=0 ; n=1
\end{gathered}
$$


Since $x_{d}$ is large ( 30 or more) $g\left(n_{d}\right)$ is near one, and the effect on $A$ is small. $g\left(n_{d}\right)$ is nearly independent of temperature over the limited range encountered in the scanning experiments (that is, does not vary noticeably with w) but does change with reaction order. For example, with $\mathrm{X}=35$, $g\left(\eta_{d}\right)=0.95,1,1.05$ and 1.10 for $n=0,1,2$ and 3 .

Using the approximation given above, it can be shown that $D$ in equation

13

$$
D=1+2 R_{d} / E
$$

becomes

$$
D=1+2 / x_{d}+2(1-n)\left(x_{d}^{-2}-6 x_{d}^{-3} \cdots\right)
$$

again introducing only a small effect on the value for $E$ derived using the ASTM method.

Each functional form for $f(n)$ must be investigated separately in order to determine whether or not the assumptions about $g(n)$ hold. Consider $f(n)$ for an autocatalytic but "nearly" simple reaction:

$$
f(n)=\left(n+n_{0}\right)(1-n)
$$

whẹre $\eta_{0}$ is small and one can neglect its temperature variation over the texperature range of the scanning runs. In place of eqs. $\mathrm{C}-30 \mathrm{a}$ and $\mathrm{C}-30 \mathrm{~b}$ one then has

$$
g\left(n_{d}\right) G\left(n_{d}\right)=-2 y \ln \left[\frac{(1-y) n_{0}}{1+y}\right]
$$

where

$$
y=\frac{g\left(\eta_{d}\right)}{1+n_{0}} ; g\left(n_{d}\right)=2 n_{d}+n_{0}-1
$$


When $f(\eta)$ has its maximum value, $2 n_{d}=1+\eta_{0}$. Then $g\left(n_{d}\right)$ is not one (e.g. if $\eta_{0}=0.05$ and $\emptyset=0$, then $g\left(\eta_{d}\right) \sim 0.16$ ) and thus $A$ derived from eq. 15 would be a factor of five ton small. However, for purposes of calculation of the explosion temperature, what is required is $A f\left(n_{d}\right)$ where $f\left(\eta_{d}\right)$ is approximately $1 / 4$. Hence, the net error in assuming that $f\left(\eta_{d}\right) \sim$ 1 and $\mathrm{Ag}\left(\eta_{\mathrm{d}}\right) \sim \mathrm{A}$ would approximately cancel in calculating a critical explosion temperature, $T_{*} \cdot$ This is crue only if $T_{*}$ lies in the range of the scanning runs and, if not, to the extent that the temperature dependence of $\eta_{0}$ can be neglected. Intuitively, it seems clear that if the temperature dependence of $\eta_{0}$ cannot be neglected, one should expect to detect a temperature dependence in a plot of $\ln \left(w / T_{d}{ }^{2}\right)$ versus $1 / T_{d}$.

The preceding analysis is consistent with all the assumptions of the test method (e.g. uniform sample temperature, simple reaction that is not autocatalytic if one wishes to determine A) and identifies the fact that at least a necessary condition on an experiment is that error in $E$ caused by deviation of $\mathrm{dT} / \mathrm{dt}$ from a constant value of $\mathrm{w}$ be less than the imprecision in $E$.

The magnitude of the change in $E$ and $A$ produced when $n \neq 1$ so $\emptyset \neq 0$ in eq. C-31a is evaluated for our experimental data on m-azidobenzoic acid in footnote 6 of table 9. The least squares program (LSTSQR) of table 9 fits eq. C-26 with $g\left(n_{d}\right)$ given by eq. C-31 directly to the experimental values of pairs of $\mathrm{w}$ and $\mathrm{T}_{\mathrm{d}}$. The results in the main part of table 9 were calculated for $\mathrm{n}=1$ (see tables 6,7 , and 8 ) so that $\emptyset=0$ for these results. The form of the equation used, which is cited at the bottom of table 6 , can be derived as follows. Taking natural logarithms of both sides of eq. C-26 and rearranging with $g\left(\eta_{d}\right)$ as given by eq. C-31 yields 


$$
\frac{1}{T}=\frac{R}{E}\left[-\ln \left(\frac{R}{E}\right)+\ln A\right]-\frac{R}{E}[\ln w+2 \ln (1 / T)-\ln (1-\emptyset)]
$$

To eliminate round-off errors in the least squares procedure the above equation was modified to

$$
\begin{aligned}
1 / T-1 / T_{0}^{-} & =a-b\left[\ln \left(w / w_{0}\right)+2 \ln \left(T_{0} / T\right)-\ln (1-\emptyset)\right] \\
a & =-1 / T_{0}+b\left[\ln b+\ln A-\ln w_{0}-2 \ln \left(1 / T_{0}\right)\right] \\
b & =R / E
\end{aligned}
$$

$1 / T_{0}$ and $\ln w_{0}$ can be selected arbitrarily; we let $1 / T_{0}$ be the average of values $1 / \mathrm{T}_{\mathrm{d}}$ and $\ln \mathrm{w}_{\mathrm{o}}$ be the average of $\ln \mathrm{w}$ of the imput experimental data. The nonlinear least squares problem was linearized using the formulation of Deming [201] using $1 / T-1 / T_{0}$ and $\ln \left(w / w_{0}\right)$ as the variables and the initial values of $\mathrm{a}$ and $\mathrm{b}$ computed assuming $\emptyset=0$. The random error in $w$ is assumed to be zero. The variance of $A$ was computed from the variances of $\mathrm{a}$ and $\mathrm{b}$ and their covariance and the differential of the relation for the parameter a given above.

\section{C.5.2. Assumption of Constant Scanning Rate in Evaluating $g\left(\eta_{d}\right)$}

The assumption of a constant scanning rate over the entire exotherm is used in equation $\mathrm{C}-27 \mathrm{a}$ and underlies the treatment defining the position of the maximum. This assumption cannot hold exactly because self-heating increases the rate of temperature rise and the amount of reaction that occurs before the peak. Only the temperature regime near $\mathrm{T}_{\mathrm{d}}$ is important. An error bound is developed below.

Equation 11 in the form

$$
\ln \left(w / T_{d}^{2}\right)=\ln \left(g\left(n_{d}\right)\right)-E / R T_{d}-\ln (E / R A)
$$


is differentiated with respect to $1 / \mathrm{T}_{\mathrm{d}}$

$$
d \ln \left(w / T_{d}{ }^{2}\right) / d\left(1 / T_{d}\right)=-E / R+\left(1 / g\left(n_{d}\right)\right)\left(d g\left(n_{d}\right) / d\left(1 / T_{d}\right)\right)
$$

The second term on the right hand side is evaluated using a procedure similar to that used earlier in this appendix for equations C-27 thru C-29 except that $G\left(n_{d}\right)$ in eq. $C-27 b$ is redefined to allow for self-heating

$$
G(n)=(A E / w R)\left[P(X)-I-P\left(X_{0}\right)\right]
$$

where the new term, I, is the self-heating correction

$$
I=(R / E) \int_{T_{0}}^{T} \exp (-E / R T) d V
$$

and $V$ is defined in eq. C-22a.

$$
T=T_{p}+\Delta T_{S T A T}-\left(\tau_{a}+\tau_{s}\right) w+V
$$

$V$ is the correction to $T_{p}$, the nominal programmed temperature, due to self heating. Integrating eq. $\mathrm{C}-35 \mathrm{~b}$ by parts yields

$$
I=\frac{R}{E}\left[V \exp \left(-x_{d}\right)-\int_{X_{0}}^{X_{d}} V \exp (-X)(-d X)\right]
$$

since $V$ at $X_{0}$ is zero in our experiments (because the heat capacity of both reactants and products are small in comparison to the heat capacity of the sample container).

Using eq. $\mathrm{C}-27$, ignoring $\mathrm{P}\left(\mathrm{x}_{0}\right)$, the analog to eq. $\mathrm{C}-29$ becomes

$$
g\left(n_{d}\right) G\left(n_{d}\right)=1-e^{x_{d}} x_{d}{ }^{2} I-\frac{2}{x_{d}}+\frac{6}{x_{d}{ }^{2}} \ldots .
$$

Assuming the general rate law for simple reaction applies, $G\left(n_{d}\right)$ in eq. C35 can be eliminated for $\mathrm{n} \neq 1$ using eq. $\mathrm{C}-30 \mathrm{a}$ to obtain:

$$
\text { - } g\left(n_{d}\right)=1-\emptyset-\psi
$$

where $\emptyset$ is defined by eqs. $C-31 b$ and $C-31 c$ and $\psi$ is

$$
\begin{array}{ll}
\psi=(1-n) e^{X_{d} x_{d}{ }^{2} I} & n \neq 1 \\
\psi=0 & n=1
\end{array}
$$


Thus, again, there is no correction to $g\left(n_{d}\right)=1$ for a first order reaction. Using eqs. C-36a, b, c, the second term on the right side of eq. C-34 becomes

$\left(1 / g\left(\eta_{d}\right)\left(d g\left(n_{d}\right)\right) d\left(1 / T_{d}\right)=\left[-d \phi / d\left(1 / T_{d}\right)-d \psi / d\left(1 / T_{d}\right)\right] /(1-\phi-\psi) \quad C-37 a\right.$ where

$$
\begin{array}{cc}
-d \phi / d\left(1 / T_{d}\right)=\frac{E}{R}(1-n) \delta_{\phi} & c-37 b \\
\delta_{\phi}=2 / X_{d}-12 / X_{d}^{3} & c-37 c \\
-d \psi / d\left(1 / T_{d}\right)=-\frac{E}{R}(1-n) \delta \psi & c-37 d \\
\delta_{\psi}=\frac{V_{d}}{T_{d}}(1-Y) X_{d}\left(1+\frac{2}{X_{d}}\right)-\left(\frac{d V}{d T}\right)_{d} & c-37 e \\
Y=\int_{X}^{X_{d} \frac{(V)}{\left(V_{d}\right)} \exp \left(-X+X_{d}\right)(-d X)} & C-37 f
\end{array}
$$

The quantity $\nabla$ is the correction for self heating.

Inserting eqs. $37 \mathrm{a}, \mathrm{b}$, and $\mathrm{d}$ into eq. C-34 yields

$$
\operatorname{den}\left(w / T_{d}{ }^{2}\right) / d\left(1 / T_{d}\right)=-\frac{E}{R}\left[1+\frac{(n-1)\left(\delta_{\psi}-\delta_{\phi}\right)}{1-\phi-\psi}\right]
$$

Thus, the effect of self heating on $g\left(\eta_{d}\right)$ for simple order reactions is to decrease $\mathrm{E}$ for $\mathrm{n}>1$, etc. An upper bound to $\delta_{\psi}$ can be obtained from eq. $\mathrm{C}-37 \mathrm{~d}$. Since $(\mathrm{dV} / \mathrm{dT})_{\mathrm{d}}$ is positive and $\mathrm{Y}$ is positive (because both $\dot{V}$ and $\mathrm{V}_{\mathrm{d}}$ are positive), one obtains

$$
\delta_{\psi} \leq \frac{V_{d}}{T_{d}} x_{d} \leq \frac{x_{d}}{T_{d}} K \frac{\left(0-0_{o}\right)}{h_{1 s}^{\prime}} d
$$

$\left(0-0_{0}\right) d$ is $0-0_{0}$ in watts at $T_{d}$. For the experimental results given in table 9 of the text one finds the maximum value of $\mathrm{x}_{\mathrm{d}} / \mathrm{T}_{\mathrm{d}}$ is at most 0.09 so that if $n<2$, one can be assured that the error in $E$ will be less than 
$2 \%$ if $V_{d}$ is always kept less than $0.2 \mathrm{~K}$ for all scanning rates (i.e. up to $20 \mathrm{kmin}^{-1}$ ). For our sample containers, $h_{1 s^{\prime}}^{\prime}$ is $\sim 20 \mathrm{mWK}^{-1}$ so if ordinate signal is kept less than $4 \mathrm{~mW}$ or $1 \mathrm{mCal} / \mathrm{sec}$. the error in will certainly be less than 2\%. Many of our experiments violate this condition but the condition of eq. - C-39 is clearly conservative.

\section{C.5.3. Corrections to Assumptions 1,2 of Table 11}

Assumption 1 is that the maximum in the observed signal corresponds to the maximum in the rate of decomposition. To determine a correction we first consider the condition that must be obeyed by the ordinate signal difference $0-\mathrm{O}_{0}$ at the maximum value of $\mathrm{dn} / \mathrm{dt}$. Then we expand $0-_{0}$ in a power series in the program temperature difference, $T_{p}-\left(T_{p}\right)_{m}$, where $\left(T_{p}\right)_{m}$ is $T_{p}$ when $0-O_{0}$ is a maximum, $\left(0-0_{0}\right)_{m}$. Inserting this relation into the condition on $0-0_{0}$ when $\mathrm{d} / \mathrm{dt}$ is a maximum determines the nominal program temperature $\left(\mathrm{T}_{\mathrm{p}}\right)_{\mathrm{d}}$ at which this $\mathrm{d} n / \mathrm{dt}$ occurs. The correction that must be added to $\left(0-0_{0}\right)_{\text {m }}$ to evaluate the self heating correction at $\left(T_{p}\right)$ is then evaluated. The latter plus $\left(T_{p}\right) d^{-\left(T_{p}\right)}$ is the correction needed for assumption 1. The correction for assumption 2 is generated as a part of the analysis for assumption 1.

Define $\tau_{s}$ by

$$
\tau_{s}=\left(c_{1}+c_{2}\right) / h_{1 s}^{\prime}
$$

where $C_{2}$ is defined by entry $2 c$ of table $C-4$

$$
c_{2}=c_{2}^{i}+\left(c_{2}^{f}-c_{2}^{i}\right) \eta ; c_{2}^{i}, c_{2}^{f} \text { independent of temperature }
$$

One can then express the rate of heat evolved by the sample, $P_{2}$, from entry $2 c$ of table $c-4$ in terms of the observed ordinate signals $0,0^{i}, 0_{0}^{i}$ corresponding to $\mathrm{S}, \mathrm{S}^{i}, \mathrm{~s}_{0}^{i}$ using the ordinate transfer function, eq. C-17a, as

$T_{\text {We assume }} D O^{i}=D O_{0}^{i}=0$ over the limited temperature range in questionn;
$D=d / d t$. 


$$
P_{2}=K\left[1+\left(\tau_{d}+\tau_{s}\right) D+\tau_{s} \tau_{d} D^{2}\right](0-0)-K\left(0_{0}^{i}-0_{o}^{i}\right)(1-n)
$$

From entry 1 of table $C-4, P_{2}$ is related to $\mathrm{Dn}$ by

$$
P_{2}=\left[+Q\left(T_{r}\right)-\left(C_{2}^{P}-C_{2}^{i}\right)\left(T_{2}-T_{R}\right)\right] D \eta
$$

The symbols of eqs. $C-40 a, b$ have been defined in Appendix C.3.2. It should be noted that $C_{2}^{p}$ is the heat capacity of all the products of the reaction and is greater than $C_{2}^{f}$, the heat capacity of the products left in the partially open sample containers in our experiments.

At the maximum of $\mathrm{D} n, \mathrm{D}^{2} \eta=0$ so that $\mathrm{DP}_{2}$ at the maximum of $\mathrm{D} \eta$, $(D n)_{d}$, is given by $\left(D P_{2}\right)_{d}$ obtained from eq. $C-40 b$

$$
\left(D P_{2}\right)_{d}=-\left(C_{2}^{P}-C_{2}^{i}\right)(D n)_{d}\left[w+\frac{K}{h_{1 s}^{1}}\left(D+\tau_{d} D^{2}\right)(0-0)\right]_{d}
$$

Differentiating eq. $C-40 a$, using again $\left(D^{2} n\right)_{d}=0$, and assuming $C_{1}$ and $\mathrm{h}_{1 \mathrm{~s}}^{\prime}$ are independent of temperature gives

$$
\begin{aligned}
\left(D P_{2}\right)_{d}= & K\left[D+\left(\tau_{d}+\tau_{s}\right) D^{2}+\tau_{s} \tau_{d} D^{3}\right]\left(0-0_{o}\right)_{d}+w\left(C_{2}^{f}-C_{2}^{i}\right)(D n)_{d}+ \\
& R\left(D C_{2} / h_{1 s}^{\prime}\right)\left(D+\tau_{d} D^{2}\right)\left(0-00_{o}\right) d
\end{aligned}
$$

Equating eqs. $C-41 \mathrm{a}$ and $\mathrm{C}-41 \mathrm{~b}$, one has for the condition on $0-0$,

$$
\left[\left(1+\frac{z^{\prime}}{h_{1 s}^{\prime}}\right) D+\left[\tau_{s}+\tau_{d}\left(1+\frac{z^{\prime}}{h_{1 s}^{\prime}}\right)\right] D^{2}+\tau_{s} \tau_{d} D^{3}\right] K(0-0)_{d}=z w
$$

where

$$
z=\left(C_{2}^{P}-C_{2}^{f}\right)(D n)_{d} ; z^{\prime}=-z+2\left(C_{2}^{f}-C_{2}^{i}\right)(D n)_{d}
$$

Equation $\mathrm{C}-42 \mathrm{a}$ indicates that $\left(0-0_{0}\right) \mathrm{d}$ occurs at a program temperature before $T_{p}=\left(T_{p}\right)_{m} \cdot$ (Both $Z$ and $Z^{\prime}$ are positive. Since $D^{2}\left(0-0_{0}\right)_{d}$ is negative for a fairly large range of $T_{p}$ below and above $\left(T_{p}\right)_{m}, D\left(0-0_{0}\right)_{d}$ must be positive rather than negative for eq. C-42a to be satisfied.) Eq. $C-42 a$ can be simplified since $z^{\prime} / h_{1 s}$ is small in comparison to one. One can show $z^{\prime} / h_{1 s}^{\prime} \ll 1$ by writing $z^{\prime} / h_{1 s}^{\prime}$ in the form 
writing $z^{\prime} / h_{1 s}^{\prime}$ in the form

$$
\frac{z^{\prime}}{h_{1 s}^{\prime}}=\left[\frac{c_{2}^{p}-c_{2}^{i}+c_{2}^{f}-c_{2}^{i}}{c_{1}}\right] f
$$

where $f$ can be approximated by

$$
f \sim\left[\frac{K\left(0-0_{0}\right)}{h_{1 s}^{\prime}}\right]\left[\frac{{ }^{\prime} C_{1}}{h_{1 s}^{\prime}}\right]\left[\frac{h_{1 s}^{\prime}}{w Q\left(T_{R}\right)}\right]
$$

For nitrocellulose, the measured value of $Q\left(T_{R}\right)$ is about $2.2 \mathrm{~kJ} \mathrm{~g}^{-1}$.

Using the numerical values for nitrocellulose in table 4, values have been calculated for $f$ in Table $c-9$; $f$ is less than 0.02 . Since $c_{2}^{i}, c_{2}^{f}$, $c_{2}^{p} \ll c_{1}$ in our experiments $z^{\prime} / h_{1 s}^{\prime} \ll 1$. The term involving $z$ cannot be neglected at this stage.

Suppose now that $0-0_{0}$ is expressed as a power series in $T_{p}$ about its maximum $\left(0-0_{0}\right)_{m}$, for $T_{p} \leq\left(T_{p}\right)_{m}$ then

$$
\mathrm{k}\left(0-0_{0}\right)=\mathrm{K}\left(0-0_{0}\right)_{m}+a_{1} \mathrm{x}-\mathrm{a}_{2} \mathrm{x}^{2}+\mathrm{a}_{3} \mathrm{x}^{3} \ldots ; \mathrm{x}=\mathrm{T}_{\mathrm{p}}-\left(\mathrm{T}_{\mathrm{p}}\right)_{\mathrm{m}}<0 \quad \mathrm{C}-43
$$

Since $D\left(0-0_{0}\right)_{m}=0, a_{1}=0$, and since $D^{2}\left(0-0_{0}\right)_{m}<0, a_{2}>0$.

Assuming that the cubic term can be dropped in eq. C-43, one obtains upon substituting eq. $\mathrm{c}-43$ into eq. $\mathrm{c}-42 \mathrm{a}$ with $\mathrm{z}^{\prime}=0$ :

$$
\left(T_{p}\right)_{d}-\left(T_{p}\right)_{m}=x_{d}=-\frac{z}{2 a_{2}}-w\left(\tau_{s}+\tau_{d}\right)
$$

Numerical values of $w\left(\tau_{s}+\tau_{d}\right)$ and $a_{2}$ are listed in table $c-9$. The values of $\mathrm{a}_{2}$ were determined from the intercept of a plot of $\left[\mathrm{K}\left(0-0_{0}\right)_{\mathrm{m}}-\mathrm{K}\left(\mathrm{O}_{-}\right.\right.$ $\left.\left.0_{0}\right)\right] /(-x)^{2}=a_{2}+a_{3}(-x) \ldots$ versus $(-x)$. To determined the condition for dropping 2 , one notes that

$$
z \approx\left(\frac{\mathrm{C}_{2}^{\mathrm{p}}-\mathrm{C}_{2}^{\mathrm{f}}}{\mathrm{C}_{1}}\right) \frac{\mathrm{fh}_{1 \mathrm{~s}}^{\prime}}{2 \mathrm{a}_{2}}
$$




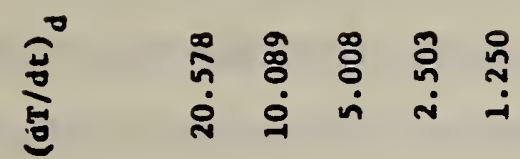

子

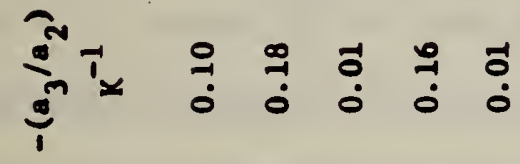

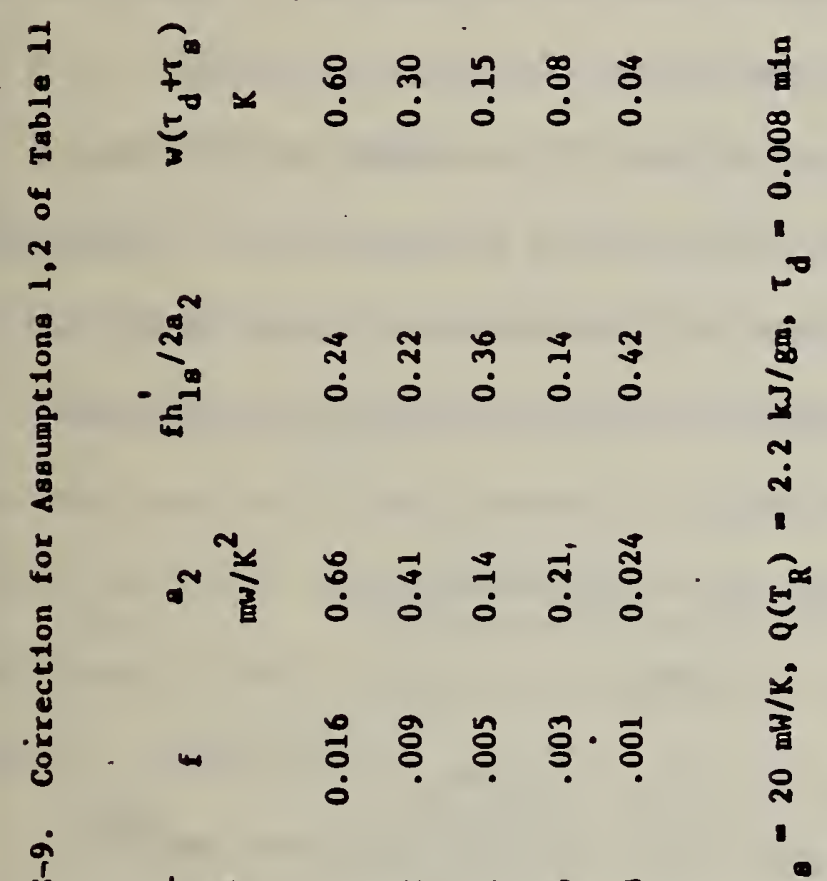

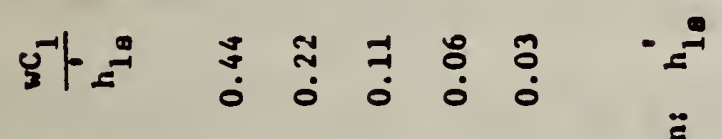

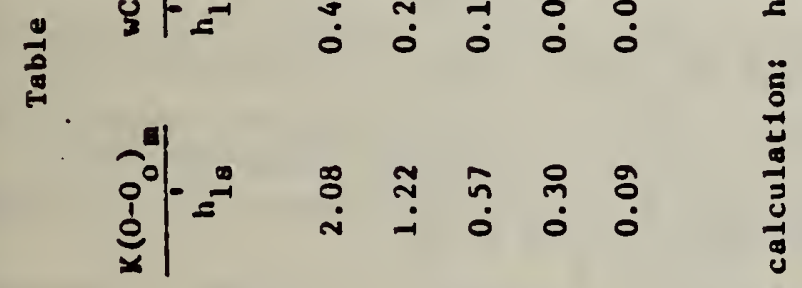

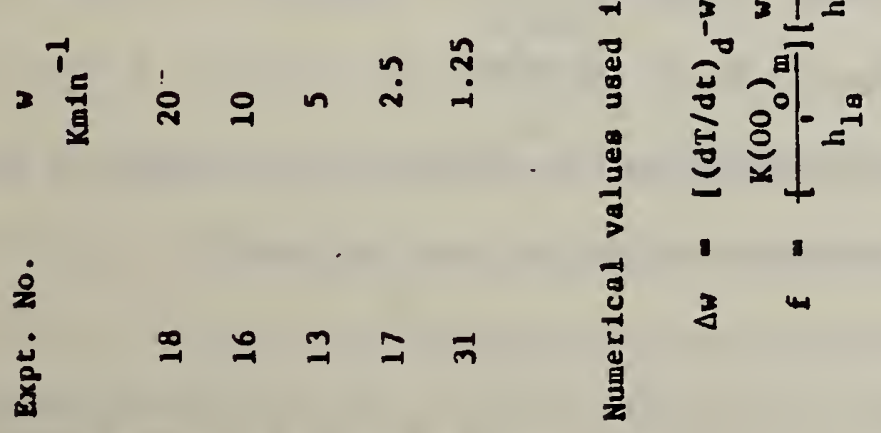


From the numerical values of $\mathrm{fh}_{1 \mathrm{~s}}^{\prime} / 2 \mathrm{a}_{2}$ in table $\mathrm{C}-9$, one concludes that for the term involving $\mathrm{Z}$ to be less than $0.05 \mathrm{~K},\left(\mathrm{C}_{2}^{\mathrm{P}}-\mathrm{C}_{2}^{\mathrm{f}}\right) / \mathrm{C}_{1}$ must be less than $0.2^{+}$. Hence, the term involving $Z$ can certainly be dropped. Approximate values of $a_{3} / a_{2}$, listed in table $c-9$, show that dropping the cubic term in eq. C-43 when determining $x_{d}$ is warranted.

The correction to the self heating term is given by

$$
\frac{k\left(0-0_{0}\right) d}{h_{1 s}^{\prime}}=\frac{k(0-0) m}{h_{1 s}^{\prime}}-\frac{a_{2}}{h_{1 s}^{\prime}}\left(w\left(\tau_{s}+\tau_{d}\right)\right)^{2}
$$

Since this leads to a maximum correction of $0.01 \mathrm{~K}$, it can be dropped in our experiments. Thus the net correction for assumption $I$ is given by eq. C-43. If we add this correction to eq. C-22c, we have the result that numerical values of $T_{d}$ determined by the ASTM method are too large by $\tau_{s} w$. From column 8 in table $C-9 \Delta T=0.4 \mathrm{~K}$ for $w=20 \mathrm{Kmin}^{-1}$. Assumption 2 is that the rate of change of temperature of the sample (at the maximum is equal to $w$. The corrected value of $(d T / d t) d$ is obtained by differentiating eq. C-22a and $c-22 b$ :

$$
\begin{aligned}
(D T)_{d} & =w+\frac{D K\left(0-0{ }_{0} d+\tau_{d} D^{2} K(0-0)^{\prime} d\right.}{h_{1 s}^{\prime}} \quad c-47 \\
& =w+\frac{2 a_{2} w^{2} \tau_{s}}{h_{1 s}^{\prime}} \cong w\left(1+\frac{2 a_{2}}{\left.h_{1 s}^{\prime} \frac{w C}{h_{l s}^{\prime}}\right)} \quad c-48\right.
\end{aligned}
$$

Numerical values of $\left(\mathrm{DT}_{2}-\mathrm{w}\right) / \mathrm{w}=\Delta \mathrm{w}$ and $\mathrm{DT}_{\mathrm{d}}$ are listed in table $\mathrm{C}-9$. It should be noted that the value of $D T,(D T)_{m}$, at $\mathrm{K}\left(00_{0}\right)_{m}$ using eq. C-47, with $\left(0-0_{0}\right)_{d}$ in place of $\left(0-0_{0}\right)_{m}$ and $D K(0-0)_{m}=0$ is

$$
(D T)_{m}=w+\frac{\tau_{d}^{2 a_{2} w^{2}}}{h_{1 s}^{\prime}}
$$

The curvature of the ordinate signal is to first approximation the same at its maximum value and at the value for the maximum in $\mathrm{d} n / \mathrm{dt}$.

\footnotetext{
For scanning rates above $1.25 \mathrm{k} / \mathrm{min}$, this is the condition that the term involving $Z$ be less than $0.1 \mathrm{w}\left(\tau_{s}+\tau_{d}\right)$ which is $.06 \mathrm{~K}$ at $20 \mathrm{~K} / \mathrm{min}$. Corrections less than $0.05 \mathrm{~K}$ are certainly not warranted in our experiments.
} 


\section{C.6. Design of a Closed Reusable Capsule}

The closed reusable capsule consists of a flat cylindrical cup and a screw cap lid made from 750-X Inconel which are sealed by means of a gold disc. The cup and lid are made from 750-X Inconel. Their dimensions are given in the cross section sketches in fig. C-9.

The gold disc is a flat plate of $0.25 \mathrm{~mm}$ thickness and $6.71 \mathrm{~mm}$ diameter. The disc is located in the top of the screw cap as indicated in $\mathrm{fig}$. C-9, (A). To place the lid in this position and also clear the internal screw threads, the disc is punched from an annealed gold sheet in the form of a shallow dish of $6.55 \mathrm{~mm}$ diameter. After annealing, the dish is placed in the inside top of the screw cap and flattened into a plate. The depth of the original gold dish is adjusted so the final diameter of the final plate just matches that required to fit the screw cap lid.

The lid is screwed on to the cup hand tight using jigs that grip the base and cup. The assembled height of the capsule is $3.49 \mathrm{~mm}$. Since the inner height of the DSC sample holder is $3.28 \mathrm{~mm}$, new sample and reference holder covers were fabricated from 0.15 mm thick platinum sheet. Dimensions are given in the cross section sketch in fig. C-10.

Further development work on the capsule is required to achieve the following three aims: (1) the capsule should have a blowout disc to prevent rupture of the cup or lid (i.e. due to overloading the cell with a material which produces gaseous decomposition products). (2) The capsule temperature shold be uniform. (3) The sample should be placed in the capsule in such a way that the temperature of the sample is kept as unfform and as close to the capsule temperature as possible.

The first of these aims can probably be accomplished by drilling a hole in the lid. The hole diameter and disc thickness would set the blowout pressure. 
The necessity of the second aim can be established as follows.

Suppose that to a good approximation the temperature of the screw cap and cup are uniform but different and that the sample temperature is uniform and equal to the cup temperature. Also, assume that the transfer of heat between the capsule cup and the sample holder lid is negligible. If $\mathrm{C}_{5}$ and $\mathrm{T}_{5}$ are the heat capacity and temperature, respectively, of the capsule lid and $C_{1}, T_{1}$ are the corresponding quantities for the capsule cup, one can show the equations for column $\# 2$ of table C-4 are valid provided the following changes are made:

a) $\mathrm{C}_{1}$ is replaced by $\mathrm{C}_{1}+\mathrm{C}_{5}$

b) $h_{i s}^{\prime}$ is defined as

$$
h_{1 s}^{\prime}=h_{1 s}+h_{\ell 5}+h_{s 5}
$$

c) $T_{S}^{\prime}$ is defined as

$$
\begin{aligned}
& T_{s}^{\prime}=T_{s}+\frac{h_{\ell 5}}{(1+r) h_{1 s}^{\prime}}\left(T_{\ell}-T_{s}\right)+\frac{\left(C_{5} D T_{5}+C_{2} D T_{2}-P_{2}\right) r}{(1+r) h_{1 s}^{\prime}} \\
& r-\left(h_{\ell 5}+h_{s 5}\right) / h_{15}
\end{aligned}
$$

$h_{1 s}$ and $h_{15}$ are the heat transfer coefficients between the cup and sample holder and the cup and capsule 1id, respectively. $h_{s 5}$ and $h_{15}$ are the heat transfer coefficients between the capsule lid and sample holder and capsule lid and sample holder lid, respectively. $T_{1}$ is the temperature of the sample holder lid. In order for the DSC temperature scale calibration to apply to a decomposition run, $r$ must be as small as possible and, thus, $h_{15} \gg h_{15}+h_{s 5^{\circ}}$. This is equivalent to making $T_{5}$ and $T_{1}$ equal.

It should be noted that one cannot prove that $r$ equals zero by showing that a plot of the onset temperature of a transition versus the scanning rate is linear. We have not found a sufficient test.

Method and tests for achieving the third aim given above have been discussed in section 5.6 . 

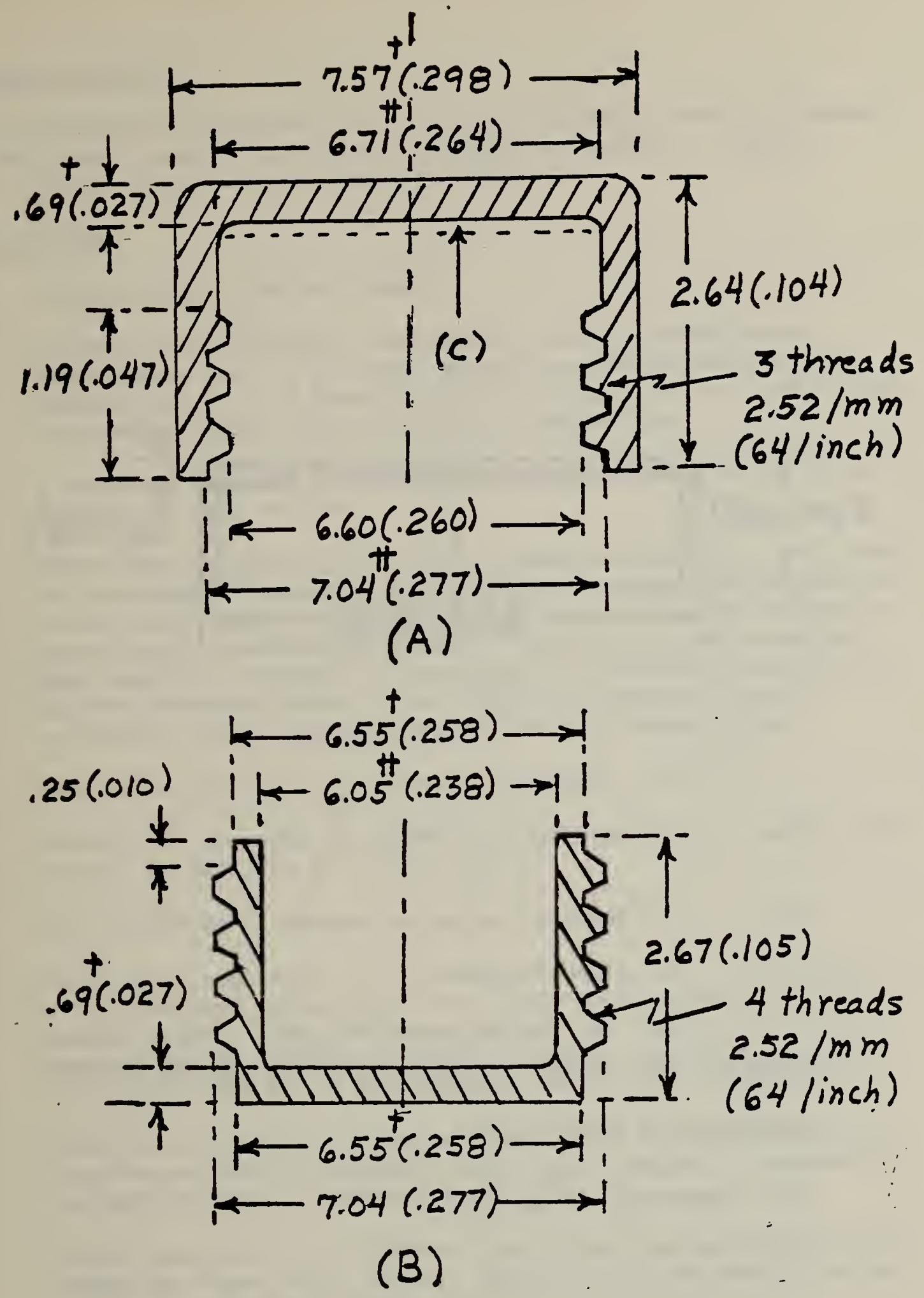

Figure C-9. Sketch of a Diametral Cross Section of Closed Reusable Capsule.

(A) Capsule screw cap (B) Capsule cup (C) Position of gold sealing disc. All dimensions in (in.) tolerance of -.00 , $+.03 \mathrm{~mm}$, tt tolerance of $+.00,-.03 \mathrm{~mm}$. 


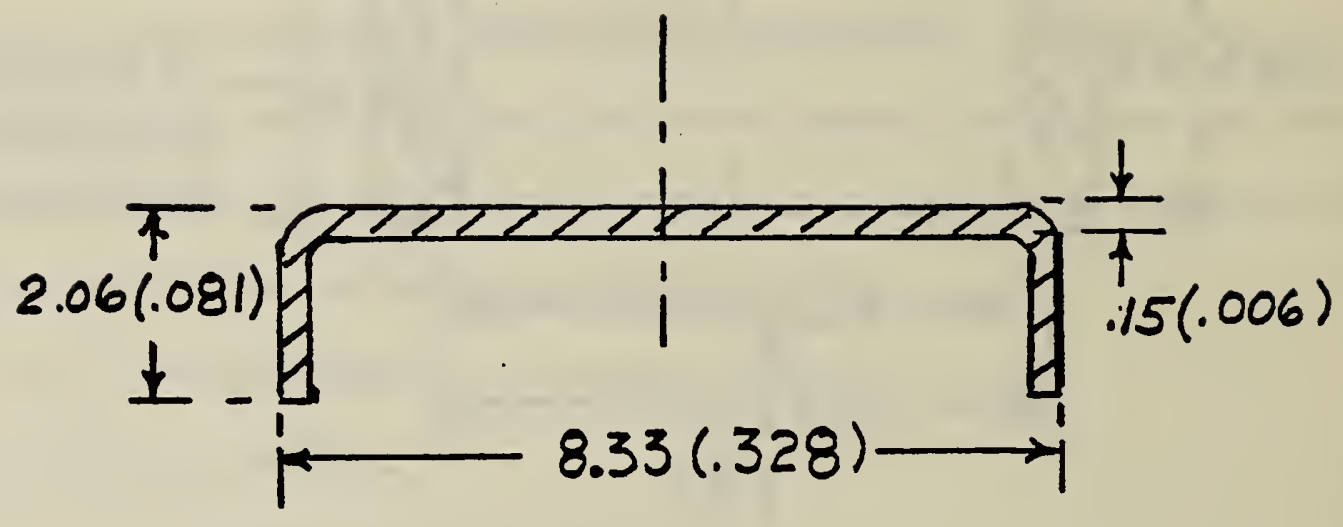

Figure $\ddot{C}-10$ Sketech of Diametral Crosṣ Section of Modified DSC Holder Lids.

All dimensions in m (in.).

C. 6-4 
Latin alphabet symbols are listed first (including symbols preceded by $\Delta$ ), the Greek symbols, and finally any special symbols. Symbols without a listed dimension are dimensionless.

\section{English Symbols}

A Preexponential factor, time $^{-1}$

Dimensionless adiabatic temperature rise: dimensionless temperature rise of material above its constant enviornment

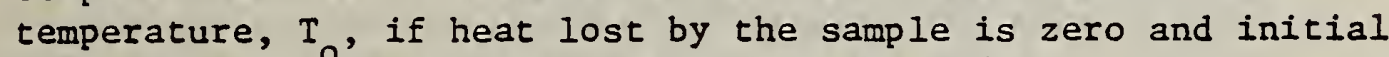
the sample temperature equals $T_{0}$. See eq. $6 a$.

$B$ when the constant enviornment temperature equals the critical value, $T_{\star}$. See eq. $6 \mathrm{~b}$.

$\mathrm{Bi}$

Biot number of material: ratio of heat transfer per unit area of the sample surface to its immediate environment divided by a mean heat transfer coefficient per unit area for conductive heat transfer within the sample. See eq. 5. The immediate environment is assumed to be either at a constant temperature in the isothermal model or to vary at a constant rate in programmed heating model (Appendix B.8); dimensionless.

Heat capacity per unit volume of material, $\mathrm{J} \cdot \mathrm{K}^{-1} \mathrm{~m}^{-3}$

Total heat capacity of material. Heat capacity of sample container in sections $5.4,5.5, \mathrm{~J} \cdot \mathrm{K}^{-1}$.

Heat capacity of sample container in Appendix $\mathrm{C} .1, \mathrm{~J} \cdot \mathrm{K}^{-1}$.

Total heat capacity; $j=1$, sample container; $j=2$, sample; $j$ $=3$, reference container; $j=4$, reference material; $j=s$, sample holder (QDTA) or sample holder cup (DSC); $j=r$, reference holder (QDTA) or reference holder cup; $j=l$, sample nolder lid (DSC); $\mathrm{j}=\mathrm{m}$, reference holder Iid (DSC); $\mathrm{J} \cdot \mathrm{K}^{-1}$.

$c_{2}^{i}$

Total heat capacity of sample just before the decomposition reaction or change in state occurs (e.g. fusion). Assumed to be independent of temperature. $\mathrm{J} \cdot \mathrm{K}^{-1}$.

$c_{2}^{f}$

Total heat capacity of sample just after the decomposition or change in state (e.g. fusion) is complete. Assumed to be independent of temperature in table $\mathrm{C}-4 . \mathrm{J} \cdot \mathrm{K}^{-1}$.

$\mathrm{C}_{2}^{\mathrm{p}}$

Total heat capacity of the products of a reaction. All of products may or may not be retained in the sample container. $\mathrm{J} \cdot \mathrm{K}^{-1}$.

Ordinary differential. Used as a subscript in section 5.2 and Appendix C.5. to denote temperature at which the rate of decomposition of the sample is a maximum. 
Ordinary differential operator with respect to time, $t, D=$ $\mathrm{d} / \mathrm{dt}$. Factor in proposed ASTM test method, see eq. 13. (Meaning clear from context.)

Arrenhius activation energy, $\mathrm{J} \mathrm{mol}^{-1}$.

$f(n) \quad$ Dependence of rate of decomposition on fraction, $n$, of a sample decomposed when sample temperature is uniform.

$f\left(n_{c}\right) \quad$ Functional dependence of $\delta_{c}^{\prime}$ or $\delta_{c}$ on reactant consumption. For sipple reactions, see eq. 9 , or see the dependence of $\left(\delta_{c} e\right)^{-1}$ of table $B-3$ on the order, $n$, of the reaction.

$f\left(n_{d}\right) \quad$ Value of $f(n)$ at the maximum value of the rate of decomposition of the sample in a programed heating experiment. .

$f\left(n_{m}\right) \quad$ Maximum value of $f(n)$.

$\mathrm{f}_{\mathrm{a}}$.

$\mathrm{f}_{\mathrm{d}}$

Proportionality façotr of the DSC average holder temperature detector (Appendix C.3.3.), $\mathrm{V} \cdot \mathrm{K}^{-1}$

Proportionality factor of the DSC differential holder temperature detector (Appendix C.3.3.). Product of the thermoelectric coefficient of the QDTA temperature difference couple divided by the gain of the difference signal amplifier (Appendix C.3.1.), $\mathrm{V} \cdot \mathrm{K}^{-1}$

f $\quad$ Adjustable proportionality factor of the DSC programer temperature (Appendix C.3.1.) $\mathrm{V} \cdot \mathrm{K}^{-1}$

$f_{i} \quad i=1,2,3$; Proportionality factors of QDTA energy measurement equation (see entry 7 of table $\mathrm{C}-3$ ).

$f_{\Delta V}$. Ratio of DSC instantaneous ordinate signal divided by output voltage supplied to sample and reference holder heaters by the differential power circuit amplifier (see entry 6 of table C5).

$g_{1}$ The ratio of the gradient of the temperature of the material at and normal to the surface of the material divided by ( $T$ $\left.T_{s}\right) / r . \quad T$ and $T_{s}$ are the average and the surface temperatures of the material, respectively, and $s$ is a characteristic dimension of the material. Heat transfer within the material is assumed to be by conduction only. (See discussion of eq. B-3la).

$g_{2} \quad$ Volume of the material divided by the product of its surface area and a characteristic dimension. (See eq. B-27).

$g\left(n_{d}\right)^{\cdot} \quad$ The negative of the derivative of $f(n)$ with respect to $n$ at the maximum rate of decomposition of the sample: $-[d f(n) / d n] d$. 
The integral: $\quad \int_{0}^{n} \mathrm{~d} n / f(n)$.

$G\left(n_{d}\right) \quad$ The value of $G(n)$ at the maximum rate of decomposition of the sample in programmed heating.

h

Heat transfer coefficient between the material and its container or environment if the temperature of the outer surface of the material and container or environment are equal. Used only in Appendix B.3. $\mathrm{W} \cdot \mathrm{K}^{-1}$

Overall heat transfer coefficient between the material and its environment in the isothermal model, Appendix B.3., in the programmed heating model (Appendix B.8.)). Heat transfer coefficient between sample and its container in adiabatic calorimetry (Appendix C.1.). $\mathrm{W} \cdot \mathrm{K}^{-1}$

Heat transfer coefficient between the sample container and its environment in adiabatic calorimetry (Appendix C.1.). $\mathrm{W} \cdot \mathrm{K}^{-1}$

Heat transfer coefficient between the surface of the material and its environment in the isothermal model, Appendix B.3.) and programmed heating model (Appendix B.8.), $\mathrm{W} \cdot \mathrm{K}^{-1}$

Heat transfer coefficient between $i$ and $j, i \neq j, h_{i j}$ is assumed to be equal to $h_{j i}$. The suoscript $i$ (or $j$ ) have the following meaning. $i=1$, sample container; $i=2$, sample; $i$ = 3, reference container; $i=4$, reference material; $i=s$, sample holder (QDTA) or reference holder cup (DSC); $i=I$, reference holder (QDTA) or reference holder cup (DSC); $i=b$, block (QDTA), $i=\ell$, sample holder cup (DSC); $i=m$, reference holder cup (DSC); $W \cdot K^{-1}$

Total heat transfer coefficient between the sample container and its environment (QDTA and DSC), $\mathrm{W} \cdot \mathrm{K}^{-1}$

Difference heat transfer coefficient of QDTA energy measurement equation (see entry 8 of table $C-3$ ), $W \cdot K^{-1}$

Total heat transfer coefficient of QDTA energy measurement equation (see entry 7 of table $\mathrm{C}-3$ ), $\mathrm{W} \cdot \mathrm{K}^{-1}$

$\mathrm{H}_{2}\left(\mathrm{~T}_{2}\right)$ Enthalpy of sample at uniform sample temperature $\mathrm{T}_{2}$, J

Io

Constant current supplied to each DSC holder by constant current generator, A.

$k(T)$ Specific rate constant of the decomposition reaction at a $k$, or uniform sample temperature $T$, time ${ }^{-1}$

k

Specific rate constant when uniform sample temperature equals to uniform constant environment temperature, time ${ }^{-1}$. 
Calibration constant of QDTA (Appendix C.3.2) or DSC (Appendix C.3.3.); $\mathrm{K}$ is temperature dependent for QDTA. Multiplication of the observed ordinate signal in volts by $K$ yields the rate of increase of energy of the reference holder plus contents minus the sample holder plus contents in units of watts; $\mathrm{W} \cdot \mathrm{V}^{-1}$. Also the unit of temperature.

Order of simple reaction, see eq. 8 .

Observed ordinate signal in a QDTA or DSC in a measurement (i.e. fusion, transition, decomposition experiment: difference between actual signal and baseline signal constructed as shown in Fig. 11, V.

$0^{k}$ $k=i, f . \quad 0$ just before (i) or after ( $j$ ) thermal event (i.e. fusion, transition, decomposition) takes place. In Appendix C. 3.4, $0^{k}$ is extrapolated, V, linearly as shown in Fig. 11.

$o_{j}$

$j=0, e$ Observed ordinate signal in a blank QDTA or DSC run with products of decomposition present in sample container (0) or with an empty sample container (e), V.

$0^{\circ}$

Observed ordinate signal in a blank QDTA or DSC run with empty sample holder, $V$.

$o_{j}^{k}$

$j=o, e ; k=i, f .0$ at temperatures just before $(k=i)$ or just after $(k=f)$ the thermal event of the corresponding measurement experiment, $V$.

$\left(0-0_{0}\right)_{m}$ The maximum $0-0_{0}$ in a decomposition run, $V$.

The rate of heat generation by the sample, $W$.

$P_{j}$

$P(X)$

$\dot{q}_{1}$

$j=A, B, D$. Components of electrical power supplied to DSC holders, see entries 1 and 2 of table $C-5, W$.

See Appendix C.5.1, eq. C-27c, X = E/RT.

Total rate of heat generation by sample in isothermal model, Appendix B.3., W.

Rate of heat loss by sample in isothermal, Appendix B.3., W.

Heat of decomposition per unit volume of sample, $\mathrm{J} \cdot \mathrm{m}^{-3}$.

Total heat of decomposition of sample, J.

Total heat of decomposition of sample referred to reference temperature $T_{R}$, J.

Rate of heat absorbed by DSC sample holder, $j=s$, or reference holder, $j=r$, from block, $W$. 
Rate of heat absorbed by DSC sample holder cup, $j=s$, sample holder 1id, $j=\ell$, reference holder cup, $j=r$, reference holder 1id, $j=m$; $W$.

Gas constant, $\mathrm{J} \cdot \mathrm{K}^{-1} \mathrm{~mol}-1$.

Resistance of electrical heater of each DSC holder, $\Omega$.

Surface area of sampie in Appendix $B, \mathrm{~m}^{2}$; instantaneous ordinate signal of DSC or QDTA (section 5, Appendices C. 3 to C.5); the observed ordinate signal lags $S$ and is related to $S$ by eq. C-17a to a good first approximation; V.

$k=i, f . \quad s$ corresponding to $0^{k}, k=i, f, V$.

$j=0, e ; s$ corresponding to $o_{j}, V$.

$\mathrm{S}$ corresponding to $0^{\circ}, \mathrm{V}$.

$j=o, e ; k=i, f . \quad s$ corresponding to $o_{j}^{k}, v$.

time, $s$.

Total time to explosion, isothermal model, Appendix B.4., s.

Time for temperature of material to rise from the environment temperature, $T_{o}$, to the explosion temperature, $T_{e}$, of the material see Appendix B.4., s.

Time for temperature of the material to rise from its initial value, $T_{\text {in' }}$, tot he environment temperature, $T_{0}$, see Appendix
B.4., S.

Temeprature of the material, $\mathrm{K}$.

Temperature of the block (QDTA), K.

Temperature of the material in isothermal model when the environment temperature, $T_{0}$, has its critical value, $T_{\star}$. See eq. $B-6, K$.

Temperature of the material at its maximum rate of decomposition, K.

Explosion temperature of the material, Appendix B.4. . Temperature of outer environment of adiabatic shield, Appendix C.1., K.

Initial uniform temperature of material at zero time of step jump case (i.e. isothermal model), Appendices B.1-7; initial uniform temperature of the material and environment, programmed heating, Appendix B. $8, \mathrm{~K}$.

Temperature of sample holder lid (DSC), K. 
Maximum value of the temperature of the material, isothermal model, Appendices B.1-B.7; temperature of the reference holder lid (DSC), Appendix C.3., K.

T Uniform temperature of sample container, Appendix C.1. only. Uniform-temperature of the environment, $K$.

$T_{\text {on }}(w)$ Observed onset temperacure (QDTA or DSC) of transition or fusion at scanning rate.w, $K$.

$T_{p}$

Instrument programmer temperature (DSC), temperature of the environment of the material in programmed heating, Appendix B. 8., K.

$\mathbf{T}_{\mathbf{r}}$

Temperature of reference holder (QDTA), reference holder cup (DSC), K.

$T_{R} \quad$ Reference temperature of the heat of decomposition, $K$.

$\mathrm{T}_{\mathrm{s}}$

Temperature of sample holder (QDTA) or sample holder cup (DSC), K.

$\mathrm{T}_{\mathrm{s}}^{\prime}$

Apparent environment temperature of sample container (QDTA, DSC), $\mathrm{K}$.

$\Delta T_{\text {STAT }}$ The static correction to be added to the abcissa or program temperature, $\mathrm{K}$.

$T_{i} \quad i=1,2,3,4$. Temperature of the sample container, sample, reference container, raference material for QDTA or DSC, K.

T * Critical explosion temperature: critical value of environment temperature, isothermal model; calculated from eqs. B-7, 8, 9 for no reactant consumption and eqs. $B-7,47,48$ for reactant consumption ( $f\left(n_{c}\right)$ would be given by eq. B-54b, for example). For the unidimensional heat conduction model, Appendix B.6., $T_{*}$ is calculated from eq. B-7, eq. B-30 divided by $f\left(\eta_{c}\right)$, and eq. B-31 with $f\left(\eta_{m}\right)$ deleted for the case of reactant consumption.

In the programmed heating model, $\mathrm{T}_{*}$ is a parameter having the same formula as $T_{*}$ for the isothermal model with reactant consumption taken into account, $K$.

Correction to the nominal programer temperature to take into account the effect of self-heating (or absorption of heat apart from heat capacity effects). See eq. C-22a, Appendix C. 3.4 ., K.

Rate of increase of environment temperature with respect to time: a) of sample environment in programmed heating, Appendix B.8. and b) of sample and reference holders in the QDTA and DSC instruments, see section 5 of text, Appendices C. 3 to C.5, $\mathrm{K} \cdot \mathrm{s}^{-1}$. 


\section{Greek Symbols}

$\alpha$

$\alpha_{e}$

$\alpha_{0}$

$\beta$

$\beta_{*}$

$\gamma$

$\gamma_{*}$

$\delta$

$\delta$

$\delta^{\prime}$

$\delta$

$\delta$

$\delta$

$\Delta$

n

$n$
Heat transfer coefficient h divided by surface area of the sample, $\mathrm{W} \cdot \mathrm{K}^{-1} \mathrm{~m}^{-2}$. Heat transfer coefficient $h_{e}$ divided by surface area of sample,
$W \cdot K^{-1} m^{-2}$.

Heat transfer coefficient $h_{0}$ divided by surface area of sample, $\mathrm{W} \cdot \mathrm{K}^{-1} \mathrm{~m}^{-2}$.

$\mathrm{RT}_{\mathrm{O}} / \mathrm{E}$.

$\mathrm{RT}_{\star} / \mathrm{E}$.

$1 / B$.

$1 / B_{\star} \cdot$

Critical parameter for isothermal model, sections B.1-B.7.

See eq. B-9 for definition for sections B.2 through B.4, eq.

B-47 for definition for section B.j.

Value of $\delta_{c}^{\prime}$ when the environment temperature is at its critical
value.

Dimensionless parameter in programmed heating model having the same formula as $\delta_{c}^{\prime}$.

Critical parameter for unidimensional heat conduction model

for thermal explosions; constant uniform environment temperature.

See eq. B-31, for no reactant consumption.

Value of $\delta$ when the environment temperature is at its critical value.

Dimensionless parameter in programmed heating model having the same formula as $\delta_{c}$.

$e-1 / \delta^{\prime}$

Fraction of initial material decomposed.

Value of $\eta$ when $f(\eta)$ is a maximum. 
Parameter in $f(\eta)$ for simple autocatalysis, see eq. B-22, p. $\mathrm{B}-14$, temperature dependence is ordinarily ignored. Actual dependence on temperature is given in footnote.

$$
\eta_{\mathrm{q}}^{\circ}
$$

K

$\lambda$

$\psi$

$\tau$

$\tau_{\mathrm{d}}$.

$\tau_{0}$

$\tau_{q}$

T

$\tau_{s}$ Fraction of material decomposed during time $t_{q}^{0}$, Appendix B.4
only.

Dimensionless temperature of material when its temperature is $\mathrm{T}$; isothermal model: $\mathrm{E}\left(\mathrm{T}-\mathrm{T}_{0}\right) / \mathrm{RT}_{0}^{2}$, programmed heating model: $\mathrm{E}\left(\mathrm{T}-\mathrm{T}_{\star}\right) / \mathrm{RT}_{\star}^{2}$.

$\theta$ when $T$ equals $T_{e}$, the explosion temperature, used in Appendix B. 3 only.

$\theta$ when $T$ equals $T_{\text {in }}$.

Maximum value of $\theta$ in isothermal model, used in Appendix B.3.

Diemsnionless temperature of environment in programmed heating model, Appendix B.8: $\mathrm{E}\left(\mathrm{T}_{\text {in }}+\right.$ wt $\left.-\mathrm{T}_{\star}\right) / \mathrm{RT}_{\star}^{2}$.

Thermal diffusivity, $\mathrm{m}^{2} \mathrm{~s}$.

Thermal conductivity, $\mathrm{WK}^{-1} \mathrm{~m}^{-1}$.

$\theta /(1+\beta \theta)$.

Time constant of the temperature $\operatorname{lag}, \tau w$, of the sample holder temperature behind the instrument programmer temperature (DSC) at a scanning rate of $\mathrm{W} \cdot \mathrm{Ks}^{-1}$; s.

Longest time constant of the differential power sircuit plus empty sample and refarence holders (DSC). The product of $\tau_{d}$ and the rate of change of the observed ordinate sigral equals the lag of the observed ordinate singla behind the instaneous ordinate signal, see eq. 17a, s.

Slope of a plot of the onset temperature of fusion or transition, $T_{\text {on }}(w)$, versus the scanning rate, w; the sum of $\tau_{a}, \tau_{d}, \tau_{s}^{i}$,
see eq. C-21c, Appendix $C .3 .3, s$.

q Thermal relaxation time of the material $\mathrm{c} / \mathrm{h}^{\prime}$ in notation of Appendices $\mathrm{B} .1$ to $\mathrm{B} .8\left(\mathrm{C}_{2} / \mathrm{h}_{1 \mathrm{~s}}\right.$ in terms of the notation of Appendix C. 3 to C.5., s. Reaction time when the sample temperature equals $T_{0} ; 1 / k_{T_{0}}$
where $k$ is the specific rate constant, $s$.

Time constant of the temperature lag, $\tau_{s} w$, of the sample container behind the sample holder temperature at a scanning rate of $\mathrm{w} \mathrm{Ks}^{-1}$ if $\mathrm{P}_{2}$ were zero,but everything else is the same (e.g. $\left.\left(C_{1}+C_{2}^{i}+\left(C_{2}^{\frac{\xi}{2}}-C_{2}^{i}\right) \eta\right) / h_{1 s}\right)$, s. 
$k=i, f_{f} \tau_{s}$ when the heat capacity of the sample is $c_{2}^{i}(k=$ i) or $C_{2}^{f}(k=f)$, respectively, $s$.

Adiabatic induction time, see eq. B-37.

Dimensionless scanning rate, programed heating model, Appendix B. 8 , see eq. B-64.

Critical value of $\Omega$. 
E. References

[ 1] Tsang, W. S., and Domalski, E. S., "An Appraisal of Methods for Estimating Self-Reaction Hazards," NBSIR 74-551, June 1974; DOT Report No. TES-20-74-8.

[ 2] Treweek, D. N., Clayton, C. R., and Seaton, W. H., Chem. Eng. Progr., Loss Prevention 7,21 (1973).

[ 3] Seaton, W. H., Friedman, E., and Treweek, D. N., ASTM Data Series Publication DS51 ASTM 1916 Race Street, Phailadelphia, PA 19103 (1974).

[ 4] Davis, A. J., and Ake, J. A., Chem. Eng. Progr., Loss Prevention $\underline{\text {, }}$ 28 (1973).

[ 5] Stull, D. R., Chem. Eng. Progr., Loss Prevention 4, 16 (1970).

[ 6] Stull, D. R., Chem. Eng. Progr. Loss Prevention 7, 67 (1973; J. Chem. Ed., 51, A21-25 (1974).

[ 7] Domalski, E. S., and Tsang, W., Proc. 4th Int. Symp. on Transport of Hazardous Cargoes by Sea and Inland Waterways, pp. 259-274, Jacksonville, FL, October 26-30, 1975 (U.S. Dept. of Transportation and U.S. Coast Guard, Washington, DC, 1975).

[ 8] Domalski, E. S., "A Second Appraisal of Methods for Estimating Self-Reaction Hazards," NBSIR 76-1149, March (1977).

[ 9] Domalski, E. S., Proc. of the Conference on the Standardization of Safety and Performance Tests for Energetic Materials, Volume 1, pp. 297-317; L. Avrami, H. J. Matsuguma, R. F. Walker, Editors, September 1977 (U.S. Army Armament Research and Development Command, Large Caliber Weapons System Laboratory, Dover, NJ, Special Publication ARLCD-SP-77004). 
[10] Engineering Design Handbook , "Principles of Explosive Behaviour," U.S. Army Material Command Pamphlet (AMCP 706-180), April 1972 (see Chapters $1,3,4,10,11$ ).

[11] Bowden, P. H., and Joffe, A., Fast Reactions in Solids, Butterworths Scientific Publications, London (1958).

[12] Frank-Kamenetskii, D. A., Diffusion and Heat Transfer in Chemical Kinetics, Second enlarged and revised edition. J. P. Appleton, Translation Editor, Plenum Press, New York (1969) (See Chapters 6, 7 , 9 in particular).

[13] Gray, P., and Lee, P. R., Thermal Explosion Theory, P. 1-183, Combustion and Oxidation Reviews, Volume 2, Elvesier Pub. Co., New York (1967) .

[14] Merzhanov, A. G., and Dubovitskii, F. I., Russian Chem. Rev., 35, 278 (1966).

[15] Thomas, P. H., "Ignition, Heat Release, and Non-Combustability of Materials," American Society for Testing Materials, STP 502, Philadelphia, PA (1972).

[16] Benson, S. W., "The Foundations of Chemical Kinetics," McGraw Hill, New York (1960). (See chapter 14).

[17] Macek, A., Chem. Rev., 62, 41-63 (1962).

[18] Merzhanov, A. G., and Averson, A. E., Combustion and Flame, 16, 89 . (1971).

[19] Berlad, A. L., Combustion and Flame 21, 275 (1973).

[20] Von Geel, J. L. C., Industrial and Eng. Chem. 58, (No. 1), 24 (1966).

[21] See ref. [10], chapter 10 and section 10-5.

[22] Merzhanov, A. G., Combustion and Flame 11, 201 (1967). 
[23] Rodgers, R. R., Thermochim. Acta 11, 131 (1975).

[24] Duswalt, A. A., "Analysis of Highly Exothermic Reactions by DSC," p. 313, "Analytical Calorimetry," R. S. Porter and J. F. Johnson, Editors, Plenum Press (1968).

[25a]Alexander, C. A., Hayland, J. R., and Treweek, D. N., "Evaluation of Computerized Techniques for Predicting Chemical Reactivity and Stability," Report No. CG-D-92-75, NTIS AD 009561 Department of Transportation, U.S. Coast Guard, April 1975.

[25b]Treweek, D. N., Pardue, W. M., Hoyland, J. R., Alexander, C. A., Seaton, W. H., and Freedman, E., "Estimation of Explosive Hazard by Computer", Ohio J. Sci. 78, 245-54 (1978).

[25b]DeHaven, E. S., "Using Kinetics to Evaluate Reactivity Hazards", Loss Prevention Symposium, 12th, Altanta, Ga., (AIChE, New York), pg. 41-44 (1978).

[26] Price, D., Chem. Rev. 59, 801 (1959).

[27] "Hazardous Chemical Data," 1973, NFPA No. 49, National Fire Protection Association, 470 Atlantic Avenue, Boston, MA 02210.

[28] Lothrup, W. C., and Handrick, G. R., Chem. Rev. 44, 419 (1949).

[29] Semenov, N. N., "Some Problems in Chemical Kinetics and Reactivity," Volume 2 (English translation by M. Boudart) Princeton University Press (1959).

[30] Todes, D. M., and Melent'ev, P. V., Zhur. Fiz. Khim. 13, 1594 (1939); 14, 1026 (1940).

[31] Bowes, P. C., Combustion and Flame 13, 521 (1969).

[32] Thomas, P. H., Trans. Far. Soc. 54, 60 (1958).

[33] Zinn, J., and Mader, C. T., J. Appl. Phys. 31, 323 (1960).

[34] Thomas, P. H., Proc. Roy. Soc. (London) A 262, 192 (1961).

[35] Rice, O. K., Allen, A. O., and Campbell, H. C., J. Am. Chem. Soc. 57, 2212 (1935). 
[36] Allen, A. O., and Rice, O. K., J. Amer. Chem. Soc. 57, 310 (1935).

[37] Campbell, H. C., and Rice, O. K., J. Amer. Chem. Soc. 57, 1044 (1935).

[38] Gray, B. F., and Sherrington, M. E., Combustion and Flame 19, 445 (1972) .

[39] Tyler, B. J., and Wesley, T. A. B., Eleventh International Symposium on Combustion, p. 1115 Pittsburgh (1967).

[40] Gray, B. F., and Sherrington, M. E., Combustion and Flame 19, 435 (1972).

[41] Adler, J., and Enig, J. W., Combustion and Flame $\underline{8}, 97$ (1964), $\underline{8}$, 342 (1964).

[42] Merzhanov, A. G., Zelikman, E. G., and Abramov, V. G., Dokl. Akad. Nauk SSSR, 180 (No. 3), 639 (1968).

[43] Gray, B. F., Combustion and Flame 21, 313 (1973).

[44] Gray, B. F., Combustion and Flame 21, 317 (1973).

[45] Gray, B. F., Combustion and Flame 24, 43 (1975).

[46] Barzykin, V. V., and Merzhanov, A. G., Dokl. Akad. Nauk. SSSR 121, 441 (1958).

[47] Bailey, P. B., Combustion and Flame 23, 329 (1974).

[48] Shouman, A. R., Donaldson, A. B., and Tsao, H. Y., Combustion and Flame 23, 17 (1974).

[49] Shouman, A. R., and Donaldson, A. B., Combustion and Flame 24, 203 (1974).

[50] Adler, J., Combustion and Flame 24, 269 (1975).

[51] Gray, P., Lee, P. R., and MacDonald, J. A., Combustion and Flame 11, 461 (1969).

[52.] Wake, G. C., Combustion and Flame 21, 119 (1973). 
[53] Hardee, H. C., Lee, D. O., and Donaldson, A. B., Combustion and Flame 18, 403 (1972).

[54] Hardee, H. C., Donaldson, A. B., and Lee, D. O., Combustion and Flame 13, 331-342 (1972).

[55] Boddington, T., Gray, P., Harvey, D. J., Phil. Trans. Roy. Soc. 270, 476 (1971).

[56] Adler, J., Combustion and Flame 24, 151 (1975).

[57] Zatushka, M. B., and Adler, J., Combustion and Flame 13, 13 (1969).

[58] Kondratiev, V. N., Chemical Kinetics of Gas Reactions, J. M. Crabtree and S. N. Carruthers, Trans., N. B. Slater, Editor, Pergamon, Oxford (1964).

[59] Merzhanov, A. G., and Dubovitskii, F. I., J. Phys. Chem. 34, (No. 10) 1062 (1960).

[60] Mielentiev, P. V., and Todes, O. M., Acta Physiochimica U.R.S.S. 14, 27 (1941).

[61] Todes. O. M., Zhur. Fiz. Khim. 13, 868 (1939); 13, 1954 (1939); 14, $1026(1940)$

[62] Merzhanov, A. G., and Dubovitskii, F. I., Dokl. Akad. Nauk. SSSR $\underline{120}, 1068$ (1958).

[63] Barzykin, V. V., Gontovskaya, V. T., and Merzhanov, A. G., Quoted in press by ref. [7] in Fiz. Goreniya Vzyrva (after 1966).

[64] Abramov, V. G., Gontovskaya, V. T., and Merzhanov, A. G., Izvest: Akad. Nauk SSSR, Ser. Khim., No. 1, 26 (1967).

[65] Khudyaev, S. I., Nauch.-Tekh. Probl. Goreniya Vzryva, No. 1, 70 (1965).

[66] Dubovitskii, F. I., Manelis, G. B., and Merzhanov, A. G., Dok1. Akad. Nauk SSSR 121, 121 (1958).

[67] Manelis, G. B., and Dubovitskii, F. I., Dokl. Akad. Nauk SSSR 126, (4), 813 (1959) 
[68] Merzhanov, A. G., and Dubovitski1, F. I., Dokl. Akad. Nauk SSSR 124, 65 (1959), 124, 362 (1959).

[69] Gray, B. F., and Yang, C. H., J. Phys. Chem. 69, 2747 (1965).

[70] Gray, B. F., and Yang, C. H., 11th Symposium on Combustion (1966), p. 1099 .

[71] Gray, B. F., Trans. Faraday Soc. 65, 1603 (1969).

[72] Yang, C. H., and Gray, B. F., Trans. Faraday Soc. 65, 1614 (1969).

[73] Gray, B. F., Trans. Faraday Soc. 65, 2133 (1969):

[74] Yang, C. H., and Gray, B. F., J. Phys. Chem. 73, 3395 (1969).

[75] Yang, C. H., J. Phys. Chem. 73, 3405 (1969).

[76] Davis, H. T., Introduction to Nonlinear Differential and Integral Equations, Dover, New York (1962).

[77] Bowes, P. C., Combustion and Flame 13, 521 (1969).

[78] Bowes, P. C., Combustion and Flame 19, 55 (1972).

[79] Walker, I. K., Harrison, W. J., Read, A. J., New Zealand Jour. Sci. 12, 302 (1969).

[80] Halstead, M. P., Prothro, A., and Quinn, C. P., Proc. Roy. Soc. (Ĺondon) A 322, 377 (1971).

[81] Lucquin, Combustion and Flame 15, 89 (1970).

[82] Merzhanov, A. G., Dokl. Akad. Nauk SSSR 140, 637 (1961).

[83] Merzhanov, A. G., and Struina, A. G., Nauch.-Tekh. Probl. Goreniya Vzryva, No. 1, 59 (1965).

[84] Struina, A. G., Merzhanov, A. G., and Maiofis, Z. B. Nauch.-Tekh. Probl. Goreniya Vzyrova, No. 2, 108 (1965).

[85] Struina, A. G., Gontovskaya, V. T., and Merzhanov, A. G., Fiz. Goreniya Vzryva, No: 3, 36 (1965). 
[86] Struina, A. G., Abramov, V. G., and Merzhanov, A. G., Fiz. Goreniya 1 Vzyrva 2, 2 (1966); Zhur. Fiz. Khim. 43, 1 (1969).

[87] Struina, A. G., Candidate's Dissertation, Institute of Chemical Physica, Academy of Sciences of the USSR (1967).

[88] Barzykin, V. V., Fiz. Goreniya i Vzyrva 9, 37 (1973).

[89] Baryzkin, V. V., Gontovskaya, V. T., Merzhanov, A. G., and Ozerkovskaya, N. I., Proceedings of the Fourth ICTA, Volume 1, Theory Inorganic Chemistry I. Buzas, Ed., Heyden and Sons Itd. (New York, 1975). [90] Grigor'ev, Yu. M., Merzhanov, A. G., and R. V. Pribytkova, Zhur. Prikl. Mekhan. L. Tekhn. Fiz. In press at time of publication of ref $[7]$.

[91] Grigor'ev, Yu. M., Maksimiov, E. I., and Merzhanov, A. G., Nauch.Tekh. Probl. Goreniya Vzryva, No. 1, 93 (1965).

[92] Ashmore, P. G., Tyler, B. J., and Wesley, T. A. B., Eleventh International Symposium on Combustion, p. 1133 Pittsburgh (1967).

[93] Gray, P., and Lee, P. R., Eleventh International Symposium on Combustion, p. 1123, Pittsburgh (1967).

[94] Fine, D. H., Gray, P., and Mackinven, R., a) Proc. Roy. Soc. London A $316 ; 223$ (1970); b) ibid. A 316,241 (1970); c) ibid. A 316,255 (1970).

[95] Ip, J. K. K., and Gray, P., Combustion and Flame 19, 117 (1972).

[96] Griffiths, J. F., Gray, P., and Kishore, K., Combustion and Flame 22, 197 (1974).

[97] Thompson, D., and Gray, P., Combustion and Flame 23, 319 (1974).

[98] Dubovitskii, F. I., Rubtsov, Yu. I., and Manelis, G. B., Izv. Akad. Nauk SSSR, Otdel Khim. Nauk 10, 1763 (1960). 
[ 99] Dubovitskii, F. I., Rubtsov, Yu. I., Barzykin, V. V., and Manelis, G. B., Izv. Akad. Nauk SSSR, Otdel Khim. Nauk 6, 1126 (1960).

[100] Dubovitshii, F. I., Barzykin, V. V., and Merzhanov, A. G., Izv. Akad. Nauk SSSR, Otdel. Khim. Nauk 6, 1124 (1960).

[101] Merzhanov, A. G., Barzykin, V. V., Abramov, V. G., and Dubovitshii, F. I., Russ. Jour. Phys. Chem. 35, 1024 (1961).

[102] Dubovitskii, F. I., Manelis, G. B., and Smirnov, L. P., Russ: Jour. Phys. Chem. 35, 255 (1961).

[103] Dubovitshii, F. I., Struinin, V. A., Manelis, G. B., and Merzhanov, A. G., Russ. Jour. Phys. Chem. 35, 148 (1961).

[104] Barzykin, V. V., and Merzhanov, A. G., Russ. Jour. ys. Chem. 38 , 1431 (1964).

[105] Samoilenko, N. G., Abramov, V. G., and Merzhanov, A. G., Russ. Jour. Phys. Chem. 41, 800 (1967).

[106] Merzhanov, A. G., Abramov, V. G., and Abramova, L. T., Russ. Jour. Phys. Chem. 41, 90 (1967).

[107]. Maksimov, E. I., Merzhanov, A. G., and Shkiro, V. M., Russ. Jour. Phys. Chem. 40, 251 (1966).

[108] Merzhanov, A. G., Abramov, V. G., and Abramova, I. T., Dok1. Akad. Nauk SSSR 171, 901 (1966).

[109] Abramova, L. T., Abramov, V. G., and Merzhanov, A. G., Russ. Jour : Phys. Chem. 43,647 (1969).

[110] Samoilenko, N. G., Vinokurov, A. A., Abramov, V. G., and Merzhanov, A. G., Russ. Jour. Phys. Chem. 44, 22 (1970).

[111] Shteinberg, A. S., Slutsker, B. M., and Merzhanov, A. G., Fiz. Gor. i Vzryva 4, 464 (1970). 
[112] Goncharov, E. P., Driatskaya, G. I., Merzhanov, A. G., and Shteinberg, A. S., Dokl. Akad. Nauk SSSR 197, 385 (1971).

[113] Merzhanov, A. G., Slutsker, A. B., and Shteinberg, A. S., Fiz. Gor. 1 Vzryva 4 , 540 (1968).

[114] Merzhanov, A. G., and Shtesse1, E. A., Fiz. Gor. 1 Vzryva 1,68 (1971).

[115] Barzykin, V. V., Shtessel, E. A., Dubovitskii, F. I., and Merzhanov, A. G., Fiz. Gor. i Vzryva I, 304 (1971).

[116] Clancey, V. J., R.A.R.D.E. Memorandum (X) 18/63, Tests Applied to Industrial Explosives, Part I., Summarized descriptions of tests.

[117] Noller, D. C., Mazurowski, S. J., Linden, G. F., De Leeuw, F. J. G., and Magel1, 0. L., Indust. Eng. Chem. $\underline{56}$ (12), 18 (1964); Paper presented at 20th Annual Meeting of the Reinforced Plastics Division, The Society of Plastics Industry Inc. 2/2/65, Chicago, IL.

[119] Bowes, P. C., Joint Fire Research Organization, Fire Research Note 626, July 1967.

[120] Bowes, P. C., Combustion and Flame 12, 289 (1968).

[121] Connor, J., R.A.R.D.E. Memorandum 15/74, The thermal decomposition of organic peroxides.

[122] Groothuizen, Th. M., Hartgerink, J. W., and Passron, H. J., Paper 10.2, pp. 239-251, ."Loss Prevention and Safety Promotion in Process Industries," C. H. Buschmann, Editor, Proceedings of the First International Loss Prevention Symposium, the Hague/Delft the Netherlands, 28=30 May 1974. Elvesier Scientific Publishing Company (1974). 
[123] Clancey, V. J., Paper 10.3, pp. 253-259, "Loss Prevention and Safety Promotion in Process Industries," see ref. 122.

[I24] Connor, J., Paper 10.4, pp. 261-270, "Loss Prevention and Safety Promotion in Process Industries," see ref. 122.

[125] Grewer, T., Paper 10.5, pp. 271-277, "Loss Prevention and Safety Promotion in Process Industries," see ref. 121.

[126] Bowes, P. C., Paper 10.6, pp. 279-285, "Loss Prevention and Safety Promotion in Process Industries," see ref. 121.

[127] Darling, J. A., Paper 10.7, pp. 287-29; "Loss Prevention and Safety Promotion in Process Industries," see ref. 121.

[128] Boyars, C., "An Evaluation of Organic Peroxide Hazard Classification Systems and Test Methods," Final Rept., NO LTR 72-63, Prepared for: Dept. of Transportation, Office of Hazardous Material (TSA20-72-1) Washington, DC 20590, February 1972.

[129] "Hazards of Chemical Rockets and Propellants Handbooks Vol. II. Solid Rocket Propellant Processing, Handling, Storage and Transportation," May 1972, AD 870258, CPIA/194.

[130] Gerlecki, C., "Fundamentals of Fire Explosion and Hazards Evalustion," AICHE Today Series, AICHE, 345 E. 47 Street, New York, NY, December 4-5, 1977.

[131] Hilado, C. J., and Clark, S. W., "Autoignition Temperatures of Organic Chemicals," Chem. Engr., p. 75, September 4, 1972.

[132] Stechkin, N. P., Jour. Res. NBS $\underline{53}, 49$ (1954).

[133] Mason, C. M., and Cooper, J. C., "Classification of Hazards of Materials -Water-Reactive Materials and Organic Peroxides," Prepared for: Dept, of Transportation, Office of Hazardous Material (TSA20-72-2) Washington, DC 20590, March 1972. 
[134] Guillet, J. E., and Meyer, F., Ind. Eng. Chem. Prod. Res. Develop. $\underline{1}$ (No. 4), 227 (1962).

[135] Kenda11, P. A., and Rosen, J. M., Rev. Sc1. Instr. 39, 992 (1968).

[136] Janswoude, J. J., and Pasman, H. J., "Determination of Kinetic Data by the Thermal Step Test," p. 249, Proceedings of the Conference on the Standardization of Safety and Performance Tests for Energetic Materials - Volume 1, Arrami, I., Matsuguma, R. J., and Walker, R. F., Editors, U.S. Army Armament and Research Development Command, Special Publication ARLCD-SP-77004, September 1977.

[137] Cezairliyan, A., and Beckett, C. W., Chapter 6 of Thermochemistry and Thermodynamics, Skinner, H. A., Editor, Butterworths (London, 1972).

[138] Wenograd, J., Trans. Faraday Soc. 57, 1612 (1961).

[139] Zinn, J., and Rodgers, R. N., Jour. Phys. Chem. 66, 2647 (1962).

[140] Henkin, H., and McGill, R., Ind. Eng. Chem. 44, 139 (1952).

[142] Raskin, W. H., and Robertson, A. F., Rev. Sci. Instr. 25, 541 (1954).

[143] Gross, D., and Robertson, A. F., Jour. Res. NBS 61, 413 (1958).

[144] Loftus, J. J., and Gross, D., NBS Report No. 6111, August 11, 1958 to Dept. of Navy, N.O.L., White Oak, MD 60921/5089/58.

[145] Loftus, J. J., and Gross, D., NBS Report No. 6548, September 23, 1959 to Dept. of Navy, N.O.L., White Oak, ID 60921/5089/58.

[146] Gross, D., and Amster, A. D., Eighth International Symposium on Combustion, pp. 728, Williams and Wilkins, Baltimore, MD (1962).

[147] Townsend, D., and Kohlbrand, H., Dow Chemical Company, Midland, MI, Private Communication. 
[148] Columbia Scientific Instrument Company, P.0. Box 9908, Austin, Texas, Advertisement literature, received February, 1978.

[149] Rodgers, R. N., "LASL Methods of Deriving Models for Predicting the Thermal Hazards and Useful Lifetimes of Explosives," p. 175, Proceedings of the Conference on Standardization of Safety and Performance Tests for Energetic Materials see ref. [136].

[150] ASTM Standard Method of Test E48774 "Constant-Temperature Stability of Chemical Materials.

[151] Burr, J. T. "The Application of Differential Thermal Analysis at Constant Temperature to Evaluate Hazardous Properties of Chemicals," p. 301, Thermal Analysis, Volume 1, Schwenker, R. F., Jr., and Garn, P. D., Editors Academic Press (New York, 1968).

[152] Duswalt, A. A., Thermochim. Acta $\underline{8}, 57$ (1974).

[153] Proposed ASTM Standard Method of Test for Determining the Arrhenius Rinetic Constants for Thermally Hazardous Materials.

[154] Thermal Analysis Newsletter No. 9, 1970, Perkin-Elmer Corp., Norwalk, CT.

[155] Deason, W. R., Koerner, W. E., and Munch, R. H., Indust. Engr. Chem. 51, 997 (1959).

[156] Cecil, O. B., and Koerner, W. E., Indust. Engr. Chem. 53, 475 (1961)

[157] Prosen, E. J., Goldberg, R. N., Staples, B. R., Boyd, R. N., and Armstrong, G. T., p. 253, "Thermal Analysis, Comparative Studies on Materials," H. Kambe and P. D. Garn, Editors, Kodanska Ltd. and Wiley and Sons (New York, 1974).

[158] Ozawa, T., J. Thermal Anal. 2, 301 (1970). 
[159] Garn, P. D., "Kinetic Investigations by Techniques of Thermal Analysis," p. 65, CRC Critical Reviews in Analytical Chemistry, September 1972.

[160] Sharp, J. H., "Reaction Kinetics," Chpt. 28 in "Differential Thermal Analysis, Vo1. 2, Applications," R. C. Mackenzie, Editor, Academic Press (New York, 1972).

[161] Flynn, J. H., and Wall, L. A., Jour. Res. NBS 70A 487 (1966).

[162] Carroll, B., and Manche, E. P., Thermochim. Acta 3, 449 (1972).

[163] Flynn, J. H., "Thermogravimetric Analysis and Differential Thermal Analysis," Chapter in "Aspects of Degradation and Stabilization of Polymers", H. H. G. Jellinck, Editor, Elvesier (New York, 1978).

[164] Collins, L. W., and Haws, L. D., Thermochim. Acta 21, 1 (1977).

[165] Merzhanov, A. G., Barzykin, V. V., Shteinberg, A. S., and Gontovskaya, V. T., Thermochim. Acta 21, 301 (1977).

[166] Wendlandt, W. W., "Thermal Methods of Analysis," 2nd Edition Wiley (New York, 1974).

[167]. Garn, P. D., "Thermoanalytical Methods of Investigation," Academic Press (New York, 1965).

[168] Mackenzie, R. C., (Editor), "Differential Thermal Analysis," Vols. $1 \& 2$ Academic Press (New York, 1972).

[169] Murray, P., and White, J., Trans. Brit. Ceram. Soc. 54, 204 (1955). [170] Kissinger, H. E., Anal. Chem. 29, 1702 (1957).

[171] Senum, G. I., and Yang, R. T., Jour. Thermal Anal. 11, 445 (1977).

[172] Reed, R. L., Weber, L., and Gottfried, B. S., Ind. Eng. Chem. Fundls. 4 , 38 (1965).

[173] Baxter, R. A., p. 65, "Thermal Analysis," Vol. 1, Schwenker, R. F., Jr. and Garn, P. D., Editors, Academic Press (New York, 1969). 
[174] U. S. Patent No. 3,732,722 May 15 (1972).

[175] Watson, E. S., O'Neill, M. J., Justin, J., and Brenner, N., Anal. Chem. 36, 1233 (1964).

[176] O'Neill, M. J., Anal. Chem. 36, 1238 (1964).

[177] O'Neil1, M. J., Anal. Chem. 38, 1331 (1966).

[178] 0'Neill, M. J., Anal. Chem. 47, 630 (1975).

[179] Gray, A. P., p. 209 "Analytical Calorimetry, Vol. I.," R. S. Porter and J. F. Johnson, Editors, Plenum Press (New York, 1968).

[180] David, D. J., Jour. Thermal Anal. $\underline{3}, 247$ (1971).

[181] Churney, K. L. West, E. D., and Armstrong, G. T., "A Cell Model for Isoperiobol Calorimeters," NBSIR 73-184 (1973).

[182] Brennan, W. P., Miller, N., and Whitwell, J. C., Ind. Eng. Chem. Fund. 8,314 (1969).

[183] Speros, D. M., p. 1191 in "Thermal Analysis, Vol. 2," Shwenher, R. F., Jr., and Garn, P. D., Editors, Academic Press (New York, 1969).

[184] Heuvel, H. M., and Lind, K. C. J. B., Anal. Chem. 42, 1044 (1970).

[185] Flynn, J. H., Thermochim. Acta $\underline{8}, 69$ (1974).

[186] Gutmann, C. M., and Flynn, J. H., Anal. Chem. 45, 408 (1973).

[187] Richardson, M. J., and Burrington, P., Jour. Thermal Anal. 6 , 345 (1974).

[188] Richardson, M. J., and Savill, N. G., Thermochim. Acta 12, 213 (1975).

[189] Flynn, J. H., p. 127, "Thermal Analysis, Vol. 1, Advances in Instrumentation," H. G. Wiedeman, Editor, Birkhauser Verlag (Basel, 1972). 
[190] Flynn, J. H., "An Analytical Evaluation of Differential Scanning Calorimetry," "Status of Thermal Analysis," 0. Menis, Editor NBS Special Publication 338, p. 119, October (1970).

[191] Flynn, J. H., p. 17 in "Analytical Calorimetry," volume 3, R. S. Porter and J. F. Johnson, Editors, Plenum Press (New York, 1974).

[192] Calvet, E., Chapter 17, "Experimental Thermochemistry," Volume II, H. A. Skinner, Editor, Interscience (New York, 1962).

[193] Brie, C. M., Gurvaich, M., and Petit J.-L., Proc. 1st Internat. Conf. Calorimetry and Thermodynamics, p. 90, Warsaw, Poland, Sept. 4 (1969).

[194] Zahra, C., Lazarde, L., and Ramanetti, R., Thermochim. Acta 6, 145 (1973).

[195] Ramanetti, R., and Zahra, C., Thermochim. Acta 12, 343 (1975).

[196] Point, R., Petit, J. L., and Gravelle, P. C., Journ. Therm. Anal. 11, 431 (1977).

[197] Barrall, E. M., II, Thermochim. Acta 5, 377 (1973).

[198] Barrall, E. M., II, and Dawson, B., Thermochim. Acta 8 , 83 (1974).

[199] Barral, E. M., II, and Johnson, J. F., Chapter 1, "Techniques and Methods of Polymer Evaluation Volume 2," Slade, P. E., Jr., and" Jenkins, L. T., Editors, Marcell Dekker (New York, 1970).

[200] - Hultgren, R., Orr, R. L., Anderson, P. D., and Kelley, K. K., "Selected Values of Thermodynamic Properties of Metals and Alloys," John Wiley and Sons (New York, 1963).

[201] Deming, W. E., Jr., "Statistical Adjustment of Data," Dover (New York, 1964).

[202] Freeberg, F. E., and Alleman, T. G., Anal. Chem. 38, 1806 (1966). 
[203] Mettler Instrument Corp., Princeton, New Jersey, Part No. 27344 and 27216 or 27343 .

[204] Perkin-Elmer Corp., Norwalk, Conn., Part No. 319-0218.

[205] Luebke, H. W., Monsanto Corp., St. Louis, Mo. Private communication, October, 1977.

[206] Schonteten, C. J. H., Bakker, S., and Pennings, A. J., Anal. Chem. 49, 523 (1977).

[207] Rodgers, R. N., Thermochim. Acta $\underline{3}, 437$ (1972).

[208] Dorko, E. A., Hughes, R. S., and Downs, C. R., Anal. Chem. 42, 253 (1970).

[209] Crossley, R. W., Dorko, E. A., and Diggs, R. L., p. 429, "Analytical Calorimetry, Vol. 2," R. S. Porter and J. F. Johnson, Editors, Plenum Press (New York, 1970).

[210] Dorko, E. A. and Crossley, R. W., J. Phys. Chem. 76, 2253 (I972).

[211] Dorko, E. A., Bryant W., and Regulinski, T. L., P. 505, "Analytical Calorimetry, Vol. 3," R. S. Porter and J. F. Johnson, Editors, Plenum Press (New York, 1974).

[212] Rodgers, R. N. and Daub, R. W., Anal. Chem. 45, 596 (1972).

[213] Peyser, P. and Bascom, W. D., P. 537, "Analytical Calorimetry Vol. 3," R. S. Porter and J. F. Johnson, Editors, Plenum Press (New . York, 1974).

[214] Ellerstein, S. M., p. 279, "Analytical Calorimetry," see ref. 24.

[215] Barret, K. E. J., J. App1. Polym. Sci. 11, 1617 (1967).

[216] Rodgers, R. N. and Morris, E. D., Anal. Chem. 38, 413 (1966). 
NBS.IIAA IREV. Q-70)

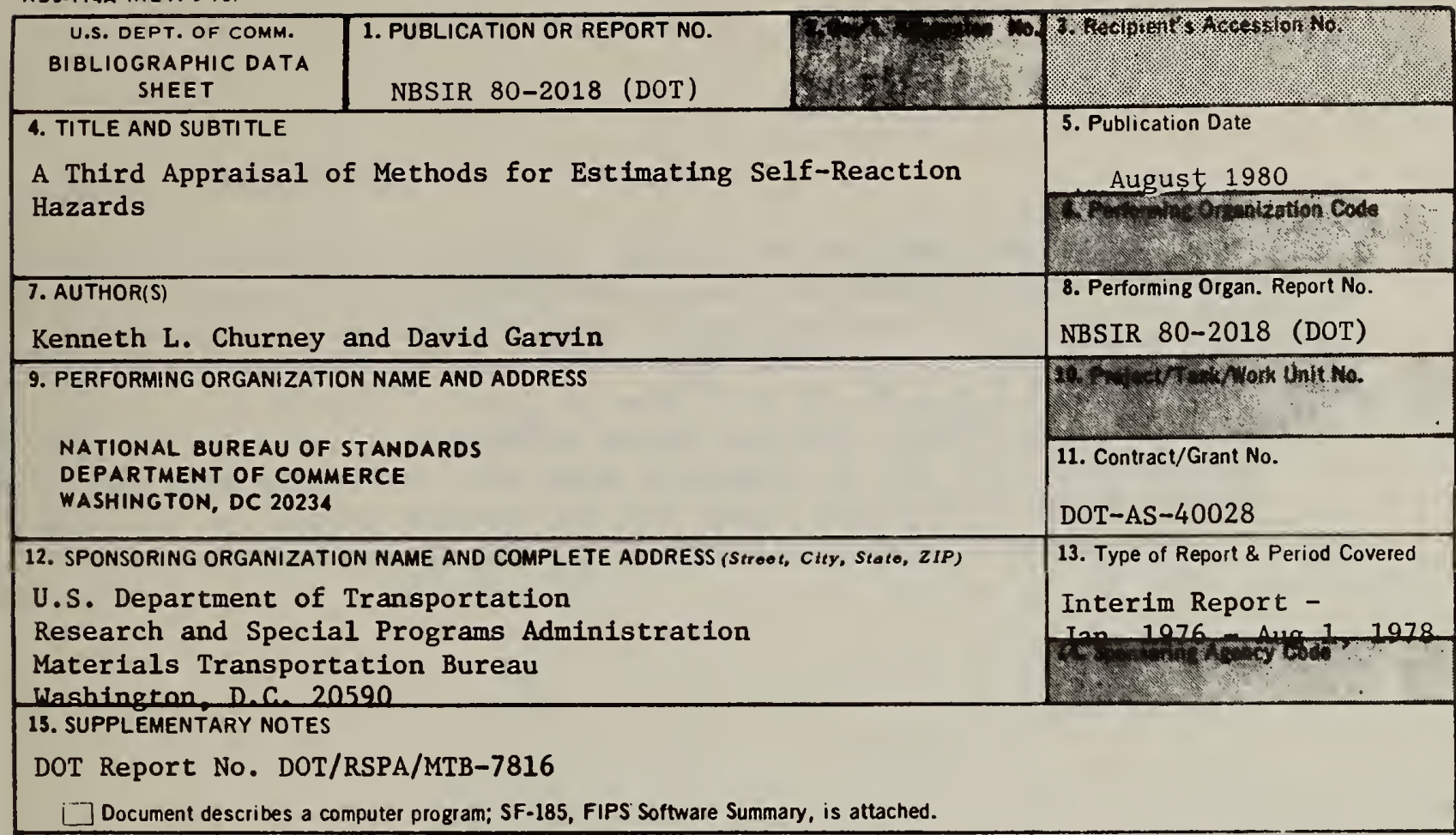

16. ABSTRACT (A 200-word or iese factual aummary of most signiftcant information. It document includes a significant bibitio iraphy or litorature survey, mention it horo.)

A study has been made of thermal explosion theory and thermal hazard tests. From this definitions have been developed, suitable for use in a transportation context, of thermally unstable materials-package configurations and thermal instability hazards. A thermally unstable materials package configuration is one: (1) composed of substances that can decompose or react with the evolution of heat, (2) for which a thermal mechanism of initiation of a temperature rise is present, and (3) in which a resulting rapid, large increase in the temperature of the material can occur. A thermal instability hazard exists if decomposition of the material-package configuration results in destruction of the package or neighboring objects or poses a safety threat. Typical destructions are explosion, rupture of package, and fire.

The properties of the material and package needed to predict the hazard are identified and methods for their measurement, using heat conduction and adiabatic calorimetry, are analyzed. It is concluded that, for least ambiguous scale-up of results to bulk shipping conditions, measurements should be made on large samples of the material under conditions such that heat transfer within the sample is large in comparison to heat transfer between the sample and its environment. Tests, such as the explosion temperature test, should be carried out to check the scaled-up hazard predictions.

An experimental study on three thermally unstable materials, nitrocellulose (I), m-azidobenzoic acid (II), and azidotriphenylmethane (III), was carried out using (con't)

17. KEY WORDS (eix to twolve ontries; alphabotical order; capitafize only the firat lotter of the first key word uniese a proper name;

soparated by eomicolone) Adiabatic calorimetry, azidotriphenylmethane, decomposition reactions, differential scanning calorimetry, heat conduction calorimetry, kinetics of heat evolution, m-azidobenzoic acid, nitrocellulose, quantitative differential thermal analysis, thermal explosion theory, thermal hazards, thermal instability.

\begin{tabular}{|c|c|c|}
\hline $\begin{array}{l}\text { 18. AVAILABILITY } \\
\square \text { For Official Distribution. Do Isot Release to NTIS }\end{array}$ & $\begin{array}{l}\text { 19. SECURITY CLASS } \\
\text { (THIS REPORT) } \\
\text { UNCLASSIFIED }\end{array}$ & $\begin{array}{l}\text { 21. NO. OF } \\
\text { PRINTED PAGES } \\
326\end{array}$ \\
\hline $\begin{array}{l}\text { [. Order From Sup. of Doc., U.S. Government Printing Office, Washington, DC } \\
\text { 20402, SD Stock No. SNO03-003- }\end{array}$ & $\begin{array}{l}\text { 20. SECURITY CLASS } \\
\text { (THIS PAGE }\end{array}$ & 22. Price \\
\hline $\begin{array}{l}\text { X Order From National Technical Information Service (NTIS), Springfield, } \\
\text { VA, } 22161\end{array}$ & UNCLASSIFIED & $\$ .8 .00$ \\
\hline
\end{tabular}





\section{ABSTRACT (continued)}

a differential scanning calorimeter (DSC). The procedure used is that of a proposed ASTM method of test for determining Arrhenius kinetic constants from the variation of the temperature of the maximum rate of heat evolution pre-exponential factors, A; are: $\Delta E=$ (I) $166.9 \pm 6.1$ (II) $139.9 \pm 5.9$, and

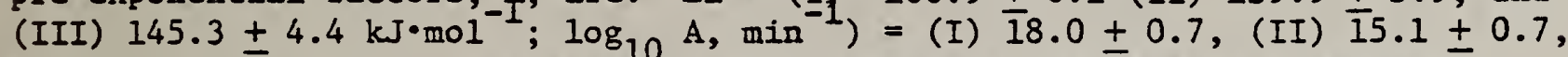
and (III) $15.0 \pm 0.6$. A systematic error analysis clarifies and tends to support the test̄ procedure. The main drawbacks to the test are its nonapplicability to complex systems and the small temperature range of the data, which makes the extrapolation of results to lower temperatures uncertain. 


